\title{
Autopsie d'une radiation adaptative : Phylogénie des Theridomorpha, rongeurs endémiques du Paléogène d'Europe - histoire, dynamique évolutive et intérêt biochronologique
}

\author{
Monique VIANEY-LIAUD* \& LAURENT MARIVAUX
}

Laboratoire de Paléontologie, Institut des Sciences de l'Évolution de Montpellier (UMR 5554, UM/CNRS/IRD/EPHE), c.c. 064, Université de Montpellier, place Eugène Bataillon, 34095 Montpellier Cedex 05, France

*auteur correspondant: Monique.Vianey-Liaud@UMontpellier.fr

Résumé : L'étude des rongeurs Theridomorpha permet de suivre le déroulement d'une radiation adaptative pendant toute sa durée (Eocène moyen-Oligocène terminal), sur un territoire restreint à l'extrémité ouest de l'Europe Occidentale. Dans ce papier, la phylogénie de ce groupe est établie à partir d'une analyse cladistique reposant sur l'examen de 315 caractères (310 dentaires). Le groupe d'intérêt comprend 110 des 132 espèces ( 24 genres) de Theridomyoidea et deux genres encore inclus jusqu'ici dans les Reithroparamyinae qui rejoignent les Theridomorpha. Les groupes externes comprennent des glires basaux, Cocomys, Tanquammys et 16 Ischyromyiformes. Un cadre phylogénétique robuste est produit, qui permet de clarifier la systématique des Theridomorpha. La position des Remyoidea (nov. supfam.) au sein des Ischyromyiformes, extérieure aux Theridomorpha, est confortée. Les Protadelomys et Tardenomys sont à la base des Theridomyoidea, avant la séparation en deux clades correspondant aux familles Pseudosciuridae et Theridomyidae. Les sous-familles sont consolidées : Pseudosciurinae et Sciuroidinae pour les Pseudosciuridae ; Issiodoromyinae, Oltinomyinae, Columbomyinae, Theridomyinae, auxquelles s'ajoute au moins une nouvelle sous-famille (Patriotheridomyinae), pour les Theridomyidae. Les chrono-espèces (sensu Simpson), traitées antérieurement comme lignées évolutives, apparaîssent dans la plupart des cas sous forme de clades successifs, émergeant dans l'ordre stratigraphique. L'analyse des caractères aux principaux nœuds permet de consolider les caractères diagnostiques des taxons et les tendances évolutives, et de discuter des divers parallélismes et convergences dans l'évolution des structures et patrons dentaires (e.g., émail des incisives unisérié chez les Issiodoromyinae et les Patriotheridomyinae, ou pseudo-multisérié chez les Blainvillimys les plus hypsodontes, les Protechimys et Archaeomys ; patrons dentaires téniodontes ; allongement des dents déciduales chez les Patriotheridomyinae, Issiodoromyinae et Theridomyidae ; sélénodontie ou lophodontie). Les dynamiques évolutives traduites par les changements morphologiques sont mises en relation avec les variations environnementales. Enfin, les implications biochronologiques de l'évolution des Theridomyoidea sont discutées.

\begin{abstract}
The adaptive radiation of the rodents Theridomorpha occurred during a limited time window (middle Eocene to late Oligocene), on an area restricted to Western Europe. In this paper, the phylogeny of this group is established via a cladistic assessment of 315 morphological characters (310 dental). The group of interest encompasses 110 upon 132 species (24 genera) of Theridomyoidea, and two genera formerly included within the Reithroparamyinae, and which are included here within the Theridomorpha. The outgroups include basal glires, Cocomys, Tanquammys and 16 Ischyromyiformes. A robust phylogenetic frame is produced, which allows clarifying the systematics of the Theridomorpha. Within the Ischyromyiformes, the Remyoidea (nov. supfam.) are set apart from the Theridomorpha. Protadelomys and Tardenomys represent the earliest offshoots of the Theridomyoidea, before the dichotomy between Pseudosciuridae and Theridomyidae. This topology supports the former subfamilies Pseudosciurinae and Sciuroidinae within the Pseudosciuridae; and for the Theridomyidae: the Issiodoromyinae, Oltinomyinae, Columbomyinae, Theridomyinae, with at least one new subfamily (Patriotheridomyinae). The chronospecies (sensu Simpson), formerly considered as evolutionary lineages, appear in most cases as successive clades emerging in the stratigraphic order. The analysis of characters supporting the main nodes allows consolidating the diagnostic characters of the taxa and their evolutionary trends, as well as discussing the various cases of parallelism and convergence in the evolution of structures and dental patterns (e.g., uniserial incisor enamel for Issiodoromyinae and Patriotheridomyinae, or pseudo-multiserial for the most hypsodont Blainvillimys, Protechimys and Archaeomys; taeniodont dental patterns; lengthening of deciduous premolars for Patriotheridomyinae, Issiodoromyinae and Theridomyidae; selenodonty or lophodonty). Evolutionary dynamics are analysed with respect to environmental changes. Finally, biochronological implications of the evolution of Theridomyoidea are discussed.
\end{abstract}

Mots-clés : Rodentia, Theridomyoidea, Diversifications, Extinction, Paléoenvironnements Keywords: Rodentia, Theridomyoidea, Diversifications, Extinction, Paleoenvironnements

Submitted 11 May 2016, Accepted 8 September 2016

Published Online 15 December 2016, doi: 10.18563/pv.40.3.e1

(C) Copyright Monique Vianey-Liaud December 2016 


\section{INTRODUCTION}

L'ordre des rongeurs témoigne d'une remarquable réussite adaptative, puisqu'il représente près de la moitié des espèces de mammifères vivant actuellement (Wilson \& Reeder, 2005). Cependant, cette image du groupe est loin de représenter l'ampleur de sa diversité depuis son origine, il y a plus de 55 millions d'années. Sur les diverses aires géographiques qu'il a colonisées, son histoire est une suite de radiations adaptatives et de transformations évolutives, ponctuée de phases d'extinctions (e.g., div. in Black \& Dawson, 1989 ; div. in Cox \& Hautier, 2015).

Les Theridomorpha Lavocat (1955) illustrent l'une de ces radiations de manière remarquable, puisque leur évolution s'est déroulée quasiment « en vase clos » (e.g., Lavocat, 1955 : 81) sur un territoire et dans un temps bien délimités. Les Theridomorpha, exclusivement paléogènes et endémiques d'Europe Occidentale, comprennent la superfamille Theridomyoidea Alston, 1876 et deux familles, Pseudosciuridae Zittel, 1893 et Theridomyidae Alston, 1876 (e.g., Comte et al., 2012). Les Theridomyoidea ont un crâne hystricomorphe, des mandibules sciurognathes et une formule dentaire comprenant $1 / 1 \mathrm{I}, 2-1 / 1 \mathrm{DP}, 1 / 1 \mathrm{P}$ et $3 / 3 \mathrm{M}$ (Fig. 1). Le plan dentaire est initialement tétralophodonte aux molaires supérieures et trilophodonte aux molaires inférieures. Il évolue vers la pentalophodontie (Stehlin \& Schaub, 1951) chez les Theridomyidae notamment et suivant des modalités et vitesses différentes en fonction des lignées au sein des deux familles. De la même façon, les prémolaires et dents déciduales sont plus ou moins molarisées, différemment selon les lignées.

Apparus au début de l'Eocène moyen, les théridomorphes deviennent les rongeurs les plus diversifiés et les plus abondants à l'Eocène supérieur, avant d'être concurrencés par les rongeurs asiatiques, immigrants de la " Grande Coupure » au début de l'Oligocène (e.g., Hartenberger, 1973a ; Lopez \& Thaler, 1975 ; Vianey-Liaud, 1979). Les espèces éocènes montrent pour la plupart des dentures à couronnes basses (i.e., brachyodontes) ou faiblement semi-hypsodontes (élévation dissymétrique des bords opposés de la couronne dentaire $=$ hypsodontie unilatérale ou asymétrique), tandis que la majorité des formes oligocènes ont des couronnes semi-hypsodontes sensiblement plus hautes. Les trois espèces qui demeurent à la fin de l'Oligocène, avant l'extinction du groupe, ont les couronnes dentaires les plus hautes. Les variations de la hauteur des couronnes ainsi que la diversité des patrons occlusaux (bunodontes, lophodontes ou sélénodontes) témoignent d'adaptations à des ressources alimentaires différentes, illustrant ainsi des spécialisations au cours du temps et des régimes alimentaires très distincts. A ces changements et diversité de structures dentaires s'ajoute un large spectre de tailles corporelles, avec des espèces qui avaient la taille d'un petit mulot ( $50 \mathrm{~g}$ ) et d'autres qui pouvaient avoir une taille équivalente à celle d'un gros rat-musqué (1 kg).

La reconstitution de leur histoire évolutive et géographique résulte des nombreuses études qui reposent sur un abondant matériel paléontologique. Bien que pour quelques taxons des fragments crâniens et mandibulaires, relativement complets, soient disponibles (e.g., Lavocat, 1951, 1955 ; Hartenberger, 1973b ; Vianey-Liaud, 1974, 1976, 1979), pour la plupart il s'agit avant tout de fragments de mâchoires et de matériel dentaire (dents isolées). Les restes dentaires, si abondants dans les gisements, ont été connus des paléontologues dès le 19 ème siècle, en France (Bassin-de-Paris, Limagne, Quercy : Aymard, 1850 ; Bravard, 1844 ; Depéret, 1917 ; Filhol, 1872a, 1872b,

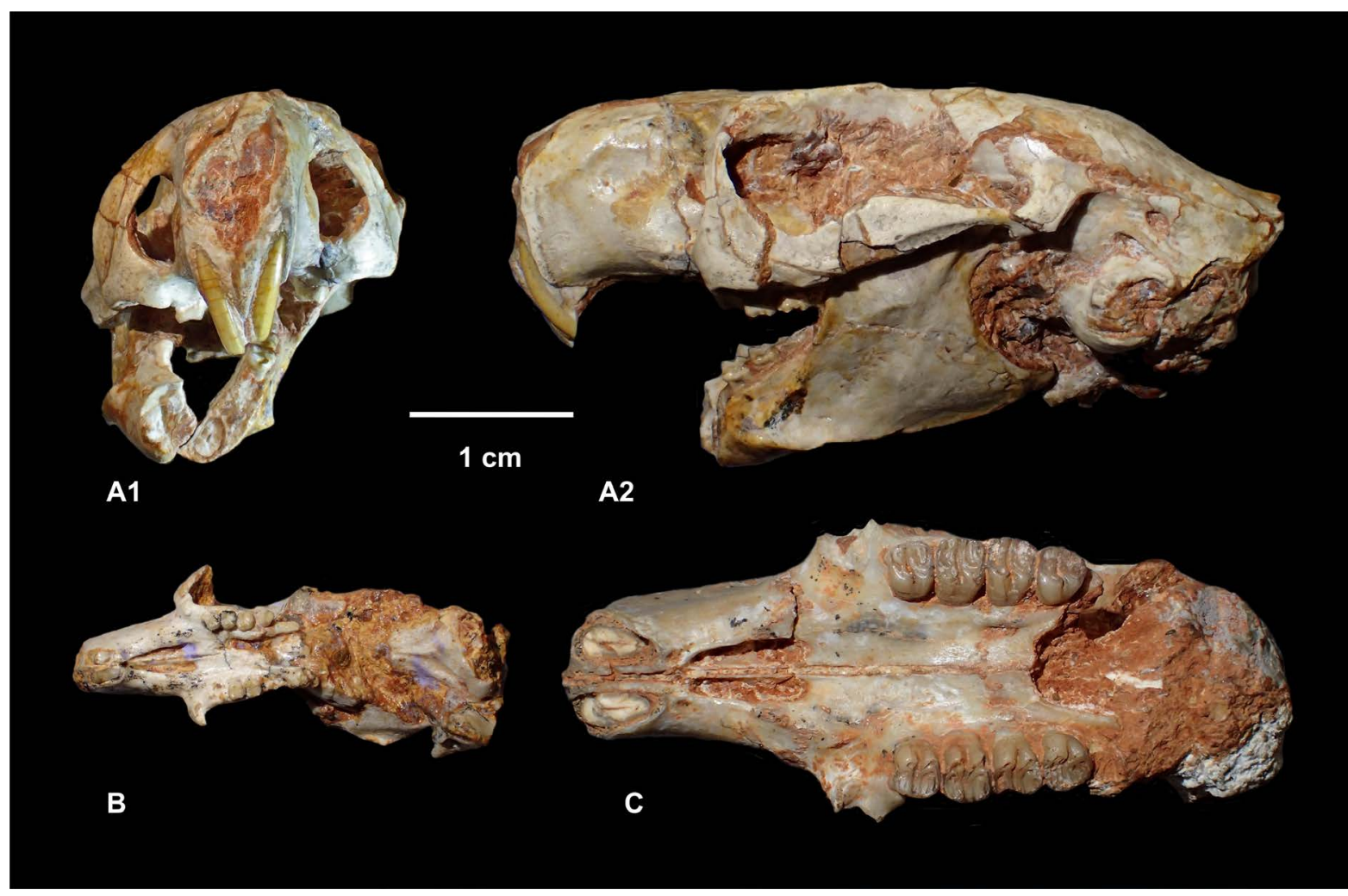

Figure 1. Fragments crâniens de quelques Theridomyoidea, donnant un aperçu de l'éventail des tailles chez ces rongeurs. A. Paradelomys crusafonti Thaler, UM-ACQ-6618, montrant le foramen infra orbitaire hystricomorphe et l'os dentaire sciurognathe, A1 : vue faciale, A2 : vue latérale gauche ; B. Elfomys sp., UMACQ-6635 (spécimen figuré par Hartenberger 1973b, PLVII, fig. 3), en vue palatine; C. Oltinomys platyceps (Filhol), UM-ACQ-6610, en vue palatine. 
1877, 1879, 1880, 1882 ; Jourdan, 1837 ; de Laizer \& de Parieu, 1839 ; Lartet, 1869 ; Pomel, 1853), en Suisse (Pictet \& Humbert, 1869 ; Major, 1873) et en Allemagne (Hensel, 1856). Si la première synthèse sur les théridomyoïdes a été écrite par Schlosser en 1884, le groupe a par la suite quitté le devant de la scène pendant plus d'un quart de siècle. Il réapparait dans la littérature à la faveur de la découverte de nouvelles faunes mammaliennes (e.g., Depéret, 1906, 1917) et ensuite à la faveur de la diffusion des idées évolutionnistes et du rôle des fossiles dans le développement de la théorie synthétique de l'évolution (e.g., Simpson, 1944). De nouvelles découvertes et la révision des données anciennes ont ranimé les études sur le groupe. Les progrès accomplis dans les méthodes d'étude, en particulier avec la prise en compte de la variabilité des espèces, rendaient nécessaire cette reprise d'autant plus que certaines espèces de Theridomyoidea ne reposent que sur un matériel des plus limité outre le type. Enfin, le contexte stratigraphique ou temporel de beaucoup des espèces anciennement décrites était inconnu. Dehm (1937), Freudenberg (1941), Tobien (1955) et Vollmayr (1966) en Allemagne, Misonne (1957) en Belgique, Stehlin \& Schaub (1951) en Suisse, Friant (1934), Lavocat (1951, 1955), Dechaseaux (1965, 1967), Thaler (1966), Hartenberger (1968, 1969), Hugueney (1969), Michaux (1968) et VianeyLiaud (1969) en France, ont abordé pour certains et développé pour d'autres, les études paléontologiques autour des rongeurs européens. Au fil des années, à la faveur de travaux de terrain rigoureux et du traitement de grandes quantités de sédiments fossilifères, ces nouvelles recherches ont permis de collecter un matériel très abondant, au sein duquel les théridomyoïdes étaient les rongeurs prédominants. Les principaux sites fossilifères concernés sont localisés en Allemagne du sud, Jura Suisse, sud de l'Angleterre et de la Belgique, sud et centre de la France ainsi qu'en Espagne. Ainsi, une série d'étude de paléontologie évolutive a été initiée sur ces rongeurs. Certains d'entre eux, parmi les Issiodoromyinae et les Theridomyinae, ont montré une évolution morphologique notable et rapide, en particulier de l'hypsodontie ou de la longueur des prémolaires déciduales, ce qui a permis de bien discriminer les espèces se succédant dans le temps. De ce fait, ils ont beaucoup contribué à la biochronologie du Paléogène ouest européen (e.g., SchmidtKittler, 1984 ; Vianey-Liaud \& Schmidt-Kittler, 1987 ; Aguilar et al., 1997).

Pour avoir une vue globale de la longue histoire des Theridomyoidea, il faut avant toutes choses se rapporter aux études diverses et d'ampleur variée dont ils ont fait l'objet. Il peut s'agir de la description de taxons comme partie intégrante de nouvelles faunes de mammifères (e.g., Depéret, 1917 ; Misonne, 1957 ; Thaler, 1969 ; Bosma, 1974 ; Bahlo, 1975 ; Hugueney, 1980 ; Peláez-Campomanes, 1996 ; Vianey-Liaud \& Schmid, 2009), d'analyses spécifiques de taxons choisis et de leur variabilité (e.g., Freudenberg, 1941 ; Gad, 1987 ; Vianey-Liaud, 1985, 2015), ou plus rarement de synthèses sur les modalités évolutives de certains taxons de Pseudosciuridae (e.g., Schmidt-Kittler, 1971 ; Hartenberger, 1971, 1973b ; Cuenca-Bescós, 1998), ou de Theridomyidae (e.g., VianeyLiaud, 1972b, 1974, 1976, 1998 ; Mödden, 1993 ; VianeyLiaud \& Ringeade, 1993 ; Schmidt-Kittler et al., 1997).

Tous les travaux cités ci-dessus ont porté en premier lieu sur les restes dentaires de ces rongeurs. Les études sur leur squelette postcrânien et leurs modes de locomotion ont été peu nombreuses. Lartet (1869) décrivait les longs os des pattes postérieures d'un théridomyidé qu'il supposait être un animal coureur (Trechomys bonduelli). Ensuite, quelques rares squelettes quasi-complets d'un theridomyidé sauteur (Issiodoromys) ont été décrits avec plus ou moins de détail (Lavocat, 1951). Sur la base de ces descriptions, le saut a été souvent considéré comme le mode de locomotion caractéristique du groupe. La révision de quelques autres squelettes, ainsi que l'étude de l'abondant matériel qu'ont livré notamment plusieurs localités du Quercy, ont conduit à une meilleure appréhension de la diversité de leurs modes de locomotion, puisque quatre types locomoteurs ont pu être reconnus, incluant le saut (Vianey-Liaud et al., 2015 ; Ginot et al., 2016) ; cependant, au vu de la diversité des patrons dentaires et crâniens, on peut s'attendre à une plus grande variété de modes de locomotion. Ainsi, beaucoup reste encore à faire, qu'il s'agisse par exemple d'une meilleure caractérisation de la variabilité dentaire de beaucoup d'espèces, ou bien de la biologie de ces taxons éteints, question peu ou pas abordée jusqu'ici [e.g., régimes alimentaires, paléoécologie, partages des niches écologiques, description et évolution des organes de l'équibre et de l'audition (oreille interne), description et évolution des patrons endocrâniens, etc.].

De façon générale, l'origine du groupe, les relations intergénériques et les découpages familiaux ou sous-familiaux ont été souvent discutés (Lavocat, 1951 ; Michaux, 1968 ; Hartenberger, 1971 ; Hooker, 1986 ; Mödden, 1993 ; VianeyLiaud et al., 1994 ; Escarguel, 1999 ; Comte et al., 2012 ; Vianey-Liaud, 2015), mais aucune analyse cladistique de l'ensemble des espèces n'a été réalisée jusqu'ici. L'analyse de Marivaux et al. (2004), qui concernait les relations intraordinales des rongeurs anciens, outre le fait qu'elle a démontré la pertinence des caractères dentaires en la matière, a soutenu l'enracinement de l'infra-ordre Theridomorpha au sein des Ischyromyoidea, comme proposé par Escarguel (1999). Par ailleurs, on peut recenser quelques analyses sommaires visant à établir la parenté (Hartenberger, 1990) ou l'origine de la super-famille (Peláez-Campomanes \& Lopez-Martinez, 1996), ou bien quelques analyses très partielles concernant les relations entre plusieurs genres de Pseudosciuridae (Hooker \& Weidmann, 2000, 2007).

Notre travail se propose de réaliser une analyse cladistique la plus exhaustive possible des théridomorphes, puisqu'elle comprend 117 des 139 espèces connues (Remyidae compris) et 15 espèces pour le groupe externe. La présente étude recense 24 genres et 132 espèces de Theridomyoidea nommés à ce jour, deux genres encore inclus jusqu'ici au sein des Reithroparamyinae, ainsi que quatre genres et sept espèces nommées de Remyidae (Tableau 1). Jusqu'ici, ces derniers étaient inclus au sein des Theridomyoidea et leur rang systématique subfamilial ou familial discuté (Hartenberger, 1973a, 1973b ; Vianey-Liaud et al., 1994 ; Quer \& Agusti, 2010 ; Comte et al., 2012); notre analyse se propose de fournir un contexte phylogénétique à cette question.

Cette analyse a nécessité une augmentation importante du nombre des caractères dentaires par rapport à celui qu'utilisaient les travaux précédents ainsi que la révision de la définition de certains d'entre eux, afin de décrire au mieux la diversité morphologique des espèces du groupe et repérer les homologies. Ce travail a pour but de mettre en évidence les relations phylogénétiques entre les différents genres afin de tester et réviser la systématique des théridomorphes. Comme tous les taxons ne sont pas définis avec la même précision, souvent du fait de la pauvreté du matériel, notre analyse permettra aussi de mettre en relief les questions d'ordre taxonomique, systématique ou phylogénétique qui demeurent, voire d'en résoudre certaines. 
PALAEOVERTEBRATA VOL.40 (3)-e1

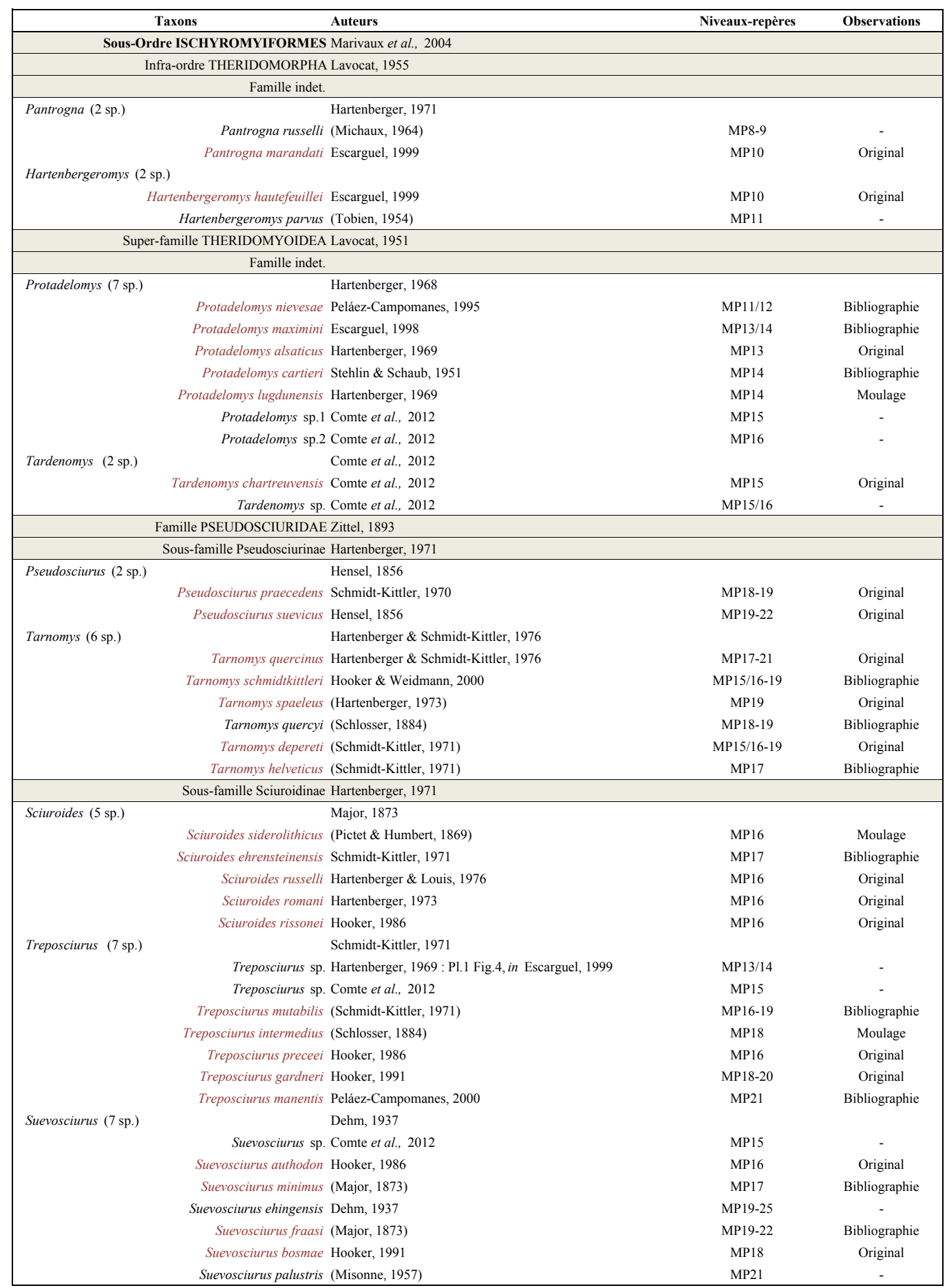

Tableau 1. Liste des taxons de Theridomorpha + Remyoidea. Le nombre de localités ayant livré les taxons étant important, sont indiquées dans ce tableau uniquement les localités - types, ainsi que le (les) niveau(x)-repère(s) correspondant à l'ensemble des localités dans lesquelles le taxon est documenté. Les taxons pris en compte dans l'analyse sont de couleur rouge. Pour les autres, la nature de l'observation est ainsi indiquée : original = d'après le matériel-type original ; moulage ; bibliographie (cf. auteur de l'espèce). 


\begin{tabular}{|c|c|c|c|}
\hline Taxons & Auteurs & Niveaux-repères & Observations \\
\hline \multicolumn{4}{|c|}{ Sous-Ordre ISCHYROMYIFORMES Marivaux et al., 2004} \\
\hline \multicolumn{4}{|c|}{ Infra-ordre THERIDOMORPHA Lavocat, 1955} \\
\hline \multicolumn{4}{|c|}{ Famille THERIDOMYIDAE Alston, 1876} \\
\hline \multicolumn{4}{|l|}{ Sous-famille indet. } \\
\hline Paradelomys (3 sp.) & Thaler, 1966 & & \\
\hline Paradelomys ruetimeyeri & (Pictet \& Humbert, 1869) & MP19 & - \\
\hline Paradelomys crusafonti & Thaler, 1966 & MP16-17 & Original \\
\hline Paradelomys santjaumensis & Salomon-Bonilla et al., 2016 & MP15 & Original \\
\hline \multicolumn{4}{|c|}{ Sous-famille Issiodoromyinae Tullberg, 1899} \\
\hline Elfomys (4 sp.) & Hartenberger, 1971 & & \\
\hline Elfomys tobieni & (Thaler, 1966) & MP15/16-16 & Original \\
\hline Elfomys parvulus & Hartenberger, 1971 & MP17-18a & Original \\
\hline Elfomys engesseri & Hooker \& Weidmann, 2007 & MP15/16 & Bibliographie \\
\hline Elfomys catalaunicus & Salomon-Bonilla et al., 2016 & MP15 & Original \\
\hline Issiodoromys (9 sp.) & Croizet, in Gervais, 1848 & & \\
\hline Issiodoromys hartenbergeri & (Vianey-Liaud \& Ringeade, 1993) & MP18b-19 & Original \\
\hline Issiodoromys medius & (Vianey-Liaud, 1976) & MP21-23 & Original \\
\hline Issiodoromys nanus & (Thaler, 1969) & MP23 & Original \\
\hline Issiodoromys minor $(1,2,3)$ & Schlosser, 1884 & MP24-25 & Original \\
\hline Issiodoromys pauffiensis & Vianey-Liaud, 1976 & MP26 & Original \\
\hline Issiodoromys quercyi & Schlosser, 1884 & MP27 & Original \\
\hline Issiodoromys limognensis & Vianey-Liaud, 1976 & MP28 & Original \\
\hline Issiodoromys pseudanaema & Gervais, 1848 & MP29 & Original \\
\hline Issiodoromys bransatensis & Hugueney, 1969 & MP30 & Original \\
\hline Pseudoltinomys (11 sp.) & Lavocat, 1951 & & \\
\hline \multicolumn{2}{|c|}{ Pseudoltinomys sp. Comte et al., 2012} & MP15 & - \\
\hline \multicolumn{2}{|c|}{ Pseudoltinomys sp. (= P. crebrum) Hooker \& Weidmann, 2000, in Comte et al., 2012} & MP15/16 & - \\
\hline \multicolumn{2}{|c|}{ Pseudoltinomys crebrum Peláez-Campomanes, 1996} & MP15 $<17 ?$ & Bibliographie \\
\hline \multicolumn{2}{|c|}{ "Pseudoltinomys" phosphoricus Hartenberger, 1973} & MP17 & Original \\
\hline \multicolumn{2}{|c|}{ Pseudoltinomys mamertensis Hartenberger, 1973} & MP16-17 & Original \\
\hline Pseudoltinomys gousnatensis & Vianey-Liaud, 1976 & MP18a & - \\
\hline Pseudoltinomys cosetanus & Hartenberger, in Anadon et al., 1983 & MP16 & Original \\
\hline Pseudoltinomys amblesi & Garzón \& López-Martinez, 1978 & MP21, 22 ou 23 & Bibliographie \\
\hline Pseudoltnomys cuvieri & (Pomel, 1852-3) & MP18-MP20 & Original \\
\hline Pseudoltinomys gaillardi & Lavocat, 1951 & MP21 & Original \\
\hline Pseudoltinomys major & Vianey-Liaud, 1976 & MP22-23 & Original \\
\hline Sous-famille Oltinomyinae & Hartenberger, 1971 & & \\
\hline Oltinomys (1 sp.) & Stehlin \& Schaub, 1951 & & \\
\hline Oltinomys platyceps & (Filhol, 1877) & MP19-20 & Original \\
\hline Ectropomys (3 sp.) & Bosma \& Schmidt-Kittler, 1972 & & \\
\hline Ectropomys exigus & Bosma \& Schmidt-Kittler, 1972 & MP18-20 & Original \\
\hline Ectropomys monacensis & Vianey-Liaud et al., 1994 & MP19-20? & Original \\
\hline Ectropomys gliriformis & (De Bruijn et al., 1979) & MP19-20? & Bibliographie \\
\hline Sous-famille Patriotheridomyinae & nov. & & \\
\hline Patriotheridomys (3 sp.) & Vianey-Liaud, 1975 & & \\
\hline Patriotheridomys sudrei & Vianey-Liaud \& Ringeade, 1993 & MP18a & Original \\
\hline Patriotheridomys altus neboulensis & Vianey-Liaud \& Ringeade, 1993 & MP18b & Original \\
\hline Patriotheridomys altus & Vianey-Liaud, 1975 & MP19-20 & Original \\
\hline Bernardia (1 sp.) & Vianey-Liaud, 1991 & & \\
\hline Bernardia marandati & Vianey-Liaud, 1991 & MP16 & Original \\
\hline Sous-famille indet. & & & \\
\hline Estellomys (4 sp.) & & & \\
\hline Estellomys nov. sp. & Comte et al., 2012 & MP15 & - \\
\hline Estellomys cansouni & Hartenberger, 1971 & MP17 & Original \\
\hline Estellomys varleti & (Hartenberger \& Louis, 1976) & MP16 & Original \\
\hline Estellomys ibericus & (Thaler, 1966) & MP15/16-16 & - \\
\hline Sous-famille Columbomyinae & Thaler, 1966 & & \\
\hline Sciuromys (4 sp.) & Schlosser, 1884 & & \\
\hline Sciuromys cayluxi & Schlosser, 1884 & MP21-22, MP23 ? & Original \\
\hline Sciuromys sp. & Lacomba \& Morales, 1987 & MP25 & - \\
\hline Sciuromys rigali & Mödden, 1993 & MP25a-b & Original \\
\hline Sciuromys quercyi & Stehlin \& Schaub, 1951 & MP indet. & - \\
\hline Columbomys (3 sp.) & Thaler, 1962 & & \\
\hline Columbomys cuencae & Hugueney et al., 1992 & MP26 & Bibliographie \\
\hline Columbomys agustii & Hugueney et al., 1992 & MP28 & Bibliographie \\
\hline Columbomys lavocati & Thaler, 1962 & MP30 & Original \\
\hline
\end{tabular}

Tableau 1. (suite). 
PALAEOVERTEBRATA VOL.40 (3)-e1

\begin{tabular}{|c|c|c|c|}
\hline Taxons & Auteurs & Niveaux-repères & Observations \\
\hline \multicolumn{4}{|c|}{ Sous-Ordre ISCHYROMYIFORMES Marivaux et al., 2004} \\
\hline \multicolumn{4}{|c|}{ Infra-ordre THERIDOMORPHA Lavocat, 1955} \\
\hline \multicolumn{4}{|c|}{ Famille THERIDOMYIDAE Alston, 1876} \\
\hline \multicolumn{4}{|c|}{ Sous-famille Theridomyinae Alston, 1876} \\
\hline Paratheridomys (3 sp.) & nov. gen. & & \\
\hline Paratheridomys margaritae & Vianey-Liaud, 1989 & MP22-MP23/24 & Original \\
\hline Paratheridomys vassoni & Pomel, 1853 & MP 22/23? & Moulage \\
\hline Paratheridomys ludensis & Vianey-Liaud, 1985 & MP24-25 & Original \\
\hline$(=$ Isoptychus bumbac & Ichensis Mayo, 1987) & MP25 & - \\
\hline Theridomys (11 sp.) & Jourdan, 1837 & & \\
\hline Theridomys brachydens & Gad, 1987 & MP19-20 & Original \\
\hline Theridomys golpei & Hartenberger, 1973 & MP18-19 & Original \\
\hline Theridomys calafensis & Anadon et al., 1987 & MP21 & Original \\
\hline Theridomys major & Depéret, 1906 & MP23 & Original \\
\hline Theridomys bonduelli & (Lartet, 1869) & MP19/20 & Original \\
\hline Theridomys aquatilis & Aymard, 1849 & MP21 & Original \\
\hline Theridomys nov. sp. (Ruch-Pouquette) & & MP22 & Original \\
\hline Theridomys sp. 1 & & $\mathrm{MP} 22 / 23$ & - \\
\hline Theridomys sp. 2 & (= T. cf. lembronicus, in Hugueney, 1994) & MP24/25 & - \\
\hline Theridomys lembronicus & Bravard, in Gervais, 1848 & MP25 & Original \\
\hline Theridomys octogesensis & Arbiol et al., 1996 & MP26-27 & Bibliographie \\
\hline Blainvillimys (16 sp.) & Stehlin \& Schaub, 1951 & & \\
\hline ? Blainvillimys sp. & Comte et al., 2012 & MP16 & - \\
\hline Blainvillimys euzetensis & (Depéret, 1917) & MP16-17 & - \\
\hline "Blainvillimys" gousnatensis & (Vianey-Liaud, 1976) & MP18a & Original \\
\hline "Blainvillimys" civracensis & Vianey-Liaud \& Ringeade, 1993 & MP18b & Original \\
\hline "Blainvillimys" pseudosiderolithicus & (De Bonis, 1964) & MP18-19 & Original \\
\hline "Blainvillimys" rotundidens & Schlosser, 1884 & MP19-20 & Original \\
\hline "Blainvillimys" langei & Vianey-Liaud, 1972 & MP21 & Original \\
\hline Blainvillimys gregarius & (Schlosser, 1884) & MP22 & Original \\
\hline Blainvillimys gemellus & Vianey-Liaud, 1989 & MP22 & Original \\
\hline Blainvillimys varians & Thaler, 1969 & MP23 & Original \\
\hline (= Blainvillimys helmeri & Vianey-Liaud, 1972) & 101125 & - \\
\hline Blainvillimys blainvillei & (Gervais, 1848) & MP25 & Original \\
\hline Blainvillimys campinsensis & (Arbiol, 1993) & MP25 & Bibliographie \\
\hline Blainvillimys heimersheimensis & Bahlo, 1975 & MP24 & Moulage \\
\hline Blainvillimys stehlini & Mayo, 1987 & MP25 & Original \\
\hline ? Blainvillimys avus & (Stehlin \& Schaub, 1951) & MP24 & Original \\
\hline Thalerimys ( $3 \mathrm{sp})$. & Tobien, 1972 & & \\
\hline Thalerimys headonensis & (Bosma, 1974) & MP17/18? & Original \\
\hline Thalerimys perrealensis & Vianey-Liaud, 1977 & MP18 & Original \\
\hline Thalerimys fordi & (Bosma \& Insole, 1972) & MP18-20? & Original \\
\hline Toeniodus (2 sp.) & Pomel, 1853 & & \\
\hline Toeniodus curvistriatus & Pomel, 1853 & MP22-MP23/24 & Original \\
\hline Toeniodus hexalophodus & Bahlo, 1975 & MP24 & Bibliographie \\
\hline Protechimys (7 sp.) & Schlosser, 1884 & & \\
\hline Protechimys variabilis & Vianey-Liaud, 1998 & MP24/25 & Original \\
\hline Protechimys truci & Hugueney, 1994 & $>$ MP23 < MP24 & Original \\
\hline Protechimys lebratierensis & Vianey-Liaud, 1998 & MP24 & Original \\
\hline Protechimys gracilis & Schlosser, 1884 & MP25 & Original \\
\hline Protechimys blainvillei & (Lavocat, 1951) & MP25 & Original \\
\hline Protechimys mayoi & Mödden, 1993 & MP indet. & - \\
\hline Protechimys major & Schlosser, 1884 & MP25/26-26 & Original \\
\hline \multicolumn{4}{|l|}{ Archaeomys / Blainvillimys (3 sp.) } \\
\hline Archaeomys / Blainvillimys sp. & in Vianey-Liaud, 2015 & MP26 & Original \\
\hline Archaeomys / Blainvillimys huerzeleri & Thaler, 1966 (syn. Bl. geminatus) & MP26-28a & Original \\
\hline Archaeomys / Blainvillimys robustus & (Lavocat, 1951) & MP26-27 & Original \\
\hline Archaeomys (8 sp.) & Laizer \& Parieu, 1839 & & \\
\hline Archaeomys arvernensis & Laizer \& Parieu, 1839 & MP29-30 & Original \\
\hline Archaeomys ehrensteini & Mödden, 1993 & MP26-26/27-27? & Bibliographie \\
\hline Archaeomys quercyi & Mödden, 1993 & MP28a & Original \\
\hline Archaeomys intermedius & Vianey-Liaud, 1979 & M 28b & Original \\
\hline Archaeomys helveticus & Vianey-Liaud, 1979 & MP28b-30 & Original \\
\hline Archaeomys laurillardi & Bravard, in Gervais, $1848-52$ & MP29 & Original \\
\hline
\end{tabular}

Tableau 1. (suite). 


\begin{tabular}{|c|c|c|c|c|}
\hline & & Auteurs & Niveaux-repères & Observations \\
\hline \multicolumn{5}{|c|}{ Sous-Ordre ISCHYROMYIFORMES Marivaux et al., 2004} \\
\hline \multicolumn{5}{|c|}{ Infra-ordre indet. } \\
\hline \multicolumn{5}{|c|}{ Super-Famille REMYOIDEA } \\
\hline \multicolumn{5}{|c|}{ Famille REMYIDAE Quer \& Agusti, 2010} \\
\hline \multirow[t]{4}{*}{ Remys (3 sp.) } & & Thaler, 1966 & & \\
\hline & Remys minimus & Hartenberger, 1973 & MP16 & Original \\
\hline & Remys garimondi & Thaler, 1966 & MP17 & Original \\
\hline & Remys major & Comte et al., 2012 & MP15-15/16 & Original \\
\hline \multirow[t]{4}{*}{ Pairomys (3 sp.) } & & Thaler, 1966 & & \\
\hline & Pairomys aff. ibericus & Vianey-Liaud et al., 1994 & MP14/15? & - \\
\hline & Pairomys ibericus & Vianey-Liaud et al., 1994 & MP15 à 17 ? & Original \\
\hline & Pairomys crusafonti & Thaler, 1966 & MP18 & Original \\
\hline \multirow[t]{2}{*}{ Frontyanamys (1 sp.) } & & Quer \& Agusti, 2010 & & \\
\hline & Frontyanamys russelli & Quer \& Agusti, 2010 & MP14-15 & Bibliographie \\
\hline \multirow[t]{2}{*}{ Zamoramys (1 sp.) } & & Peláez-Campomanes \& López-Martinez, 1996 & & \\
\hline & Zamoramys extraneus & Peláez-Campomanes \& López-Martinez, 1996 & MP11-12 ? & Bibliographie \\
\hline
\end{tabular}

Tableau 1. (suite).

Les études antérieures ont reconnu des successions temporelles d'espèces apparentées, considérées comme stades successifs de lignées évolutives au sens des chrono-espèces de Simpson (e.g., Simpson, 1951 ; Thaler, 1966 ; Schmidt-Kittler, 1971 ; Vianey-Liaud, 1975, 1976, 1998 ; Vianey-Liaud \& Schmidt-Kittler, 1987). Aussi, est-il important de rechercher l'organisation phylogénétique de ces lignées évolutives, telle qu'elle ressort de l'analyse cladistique des taxons spécifiques qui les constituent. Les représentations obtenues seront comparées aux interprétations antérieures.

Une synthèse sur les modalités et tendances évolutives (des patrons dentaires essentiellement) de l'ensemble du groupe sera aussi présentée, en relation avec les variations paléogéographiques et paléoenvironnementales, en soulignant l'intérêt biochronologique de ces rongeurs.

\section{CADRE PALÉOGÉOGRAPHIQUE ET GÉOCHRONO- LOGIQUE}

Les Theridomorpha sensu Lavocat (1955) ont évolué sur un territoire restreint pendant près de 20 millions d'années (Ma), depuis le début de l'Eocène moyen jusqu'à la fin de l'Oligocène. Sur leur aire géographique très morcelée, d'abord insulaire puis péninsulaire, ces rongeurs ont réalisé au moins trois radiations adaptatives, et subi plusieurs crises d'extinction. Leur histoire a été fortement marquée par les variations climatiques et géographiques, notamment liées aux effets des orogénèses Pyrénéenne et Alpine, qu'a connues l'Europe Occidentale. Par ailleurs, du fait de l'achèvement de l'ouverture de l'Atlantique Nord après l'Eocène inférieur, cette aire géographique a coupé les ponts avec l'Amérique du Nord. Du côté oriental, les îles européennes ont été séparées du reste de l'Eurasie par des étendues marines (au nord et au sud) jusqu'à ce que des ponts continentaux se forment, à la transition Eocène-Oligocène (e.g., Dercourt et al., 1993 ; Meulenkamp \& Sissingh, 2003 ; Schettino \& Turco, 2011 ; Kócsis et al., 2014) (Fig. 2). Cette histoire s'inscrit aussi dans la phase de baisse des températures amorcée à partir des optimums climatiques de l'Eocène inférieur. Outre quelques rares épisodes d'accroissement des températures durant l'Eocène et notamment au tout début du Bartonien (MECO ; Fig. 3), au début du Priabonien et au Chattien supérieur, plusieurs événements froids (Oi ; Fig. 3) sont enregistrés dans les sédiments marins, le premier et le plus important à la limite Eocène-Oligocène (Oi1) et plusieurs autres au cours de l'Oligocène, du Rupélien jusqu'au milieu du Chattien (e.g., Cramer et al., 2009 ; Vandenberghe et al., 2012).

Le cadre chronologique de ce travail est la «Global Time Scale » (GTS) de Gradstein et al. (2012), présentée par Vandenberghe et al. (2012). L'âge des limites d'étages au sein de l'Eocène et de l'Oligocène a subi des modifications depuis Ogg et al. (2008) : la limite Thanétien/Yprésien $=56 \mathrm{Ma}$ au lieu de 55.8 Ma ; Yprésien/Lutétien $=47.8$ au lieu de 48.6 ; Lutétien/ Bartonien $=41.2$ au lieu de $40.4 ;$ Bartonien/Priabonien $=37.8$ au lieu de 37.2 ; Rupélien/Chattien $=28.1$ au lieu de 28.4. Il convient de rappeler ici que jusqu'à la fin des années 1970, les gisements fossilifères actuellement corrélés avec le Priabonien étaient considérés comme Oligocène inférieur (e.g., Cavelier, 1965 ; Vianey-Liaud, 1979).

La figure 2 situe les gisements renfermant les associations d'espèces de Theridomyoidea correspondant à différents niveaux-repères (NR) mammaliens du Paléogène (MP) sur trois paléogéographies successives (Bartonien-Priabonien, Rupélien inférieur et Chattien supérieur). Le tableau 1 liste les différents taxons, parmi lesquels ceux qui ont fait l'objet de nos analyses phylogénétiques sont mis en relief. La liste des MP et des localités rapprochées est indiquée dans le tableau 2.

La figure 3 présente le cadre chronologique en regard duquel sont situés les niveaux-repères mammaliens du Paléogène qui permettent de repérer dans le temps les différents taxons, les variations globales de température (Cramer et al. 2009), ainsi que les variations comparées de la diversité des Theridomorpha et des Rodentia en général (cf. p. 9).

\section{ANALYSE PHYLOGÉNÉTIQUE}

\section{Matériel et méthodes}

Compte tenu du grand nombre de taxons et de localités les ayant livrées, les informations concernant la position géographique et chronologique des différentes espèces ne sont pas regroupées sur un unique tableau, celui-ci s'étant avéré trop volumineux pour être aisément lisible. Elles sont réparties et listées de façon exhaustive dans les paragraphes correspondants du chapitre «Systématique », tandis que les tableaux 1 à 3 apportent des éléments partiels. Lorsqu'il s'agit d'anciennes collections 
dont la localisation géographique et/ou stratigraphique pose question, cet état de fait est indiqué par l'abréviation A.C. (e.g., Quercy, A.C.).

La plus grande partie du matériel qui documente les 132 espèces considérées ici a été observée directement (spécimens originaux et moulages), la part des illustrations issues de la littérature restant fort réduite (cf. Tableaux 1 et 3). Pour la majorité des espèces, nous avons considéré uniquement le matériel type, afin d'être assurés de l'homogénéité temporelle du taxon. Ce fait n'exclut pas la prise en compte de la variabilité des espèces, celle-ci entrant dans la définition de nombre d'entre-elles. Nous avons analysé essentiellement les caractères dentaires (car. 5 à 315), dans la mesure où beaucoup des taxons considérés ne sont connus que par leurs dents. Une partie de ces caractères sont ceux proposés et utilisés antérieurement (e.g., Marivaux et al., 2004). Cependant, certains ont été réinterprétés et plusieurs autres caractères et/ou états de caractères ajoutés ou modifiés, afin de décrire au mieux la morphologie dentaire de l'ensemble des théridomorphes. Les caractères de la microstructure de l'émail n'ont pas été pris en compte ici, dans la mesure où trop peu de taxons pouvaient être codés. Toutefois, ils ont été cartographiés a posteriori sur la topologie obtenue. Pour la même raison, seulement quatre caractères crâniens (car. 1 à 4 ) ont été considérés ; quelques autres seront indiqués a posteriori sur la phylogénie obtenue.

Afin de repérer le plus clairement possible les homologies morphologiques, nous avons retenu les caractères des cuspides principales et accessoires aisément repérables dans le patron dentaire, leur taille, leur forme et leurs connections (lophes et lophides ; cingulums et cingulides), ainsi que les vallées les séparant (sinus et sinusides ; synclinaux et synclinides). Deux caractères (car. 5, 6) sont relatifs au degré d'hypsodontie. Celui-ci est donné par le rapport de la plus grande hauteur de la couronne sur la longueur mésio-distale de la surface occlusale (pour des dents très peu usées ; e.g., Vianey-Liaud, 2015). La taille globale n'est pas considérée, ce caractère n'ayant qu'une faible valeur phylogénétique : seule la taille relative des dents des différents loci est prise en compte (car. 12, 63, 121-122, 193-197) pour décrire soit l'accroissement antérieur, soit le raccourcissement postérieur des rangées dentaires.

Nous avons combiné les terminologies de Wood \& Wilson (1936), Stehlin \& Schaub (1951), Marivaux et al., (2004) et Comte et al. (2012) (Fig. 4).

La matrice de caractères comprend au total 315 caractères, dont certains sont binaires et d'autres à états multiples (voir Informations Supplémentaires disponibles en ligne). Pour ces derniers, ceux correspondant à une suite additive d'états (transformations morphoclinales) ont été considérés et traités comme ordonnés («ordered»). Considérer une telle hypothèse ad hoc sur les changements d'états de caractères n'est en aucun cas un jugement a priori sur la polarité des transformations des états de caractères (i.e., pour un caractère considéré comme une suite additive d'états, aucun état n'est considéré a priori comme plésiomorphe [primitif] ou apomorphe [dérivé]; voir Marivaux et al., 2004). Cependant, pour équilibrer l'influence des caractères ordonnés en termes de changements évolutifs par rapport aux caractères non-ordonnés (binaires ou à états multiples non additifs), les caractères à états multiples additifs ont été ajustés : le nombre de changements d'états de caractères a été pris en compte afin que la somme des changements (nombre de pas) d'un morphocline soit égale à « 1 ». Les caractères nonpréservés versus non-applicables ont été respectivement codés par $« ? »$ et $«-»$.
Taxons sélectionnés. Comme l'un de nos objectifs était d'établir la structure phylogénétique des théridomorphes et de leurs groupes frères, le groupe externe inclut les Glires les plus anciens (Archetypomys et Tribosphenomys), trois rongeurs basaux comprenant deux cténodactyloïdes (Cocomys et Tanquammys) et l'ischyromyoïde Acritoparamys. Le groupe de branchement comprend aussi dix autres ischyromyoïdes, incluant les genres supposés être groupes frères des théridomyoïdes Hartenbergeromys et Pantrogna (Escarguel, 1999 ; Marivaux et al., 2004). Les espèces des genres Remys,

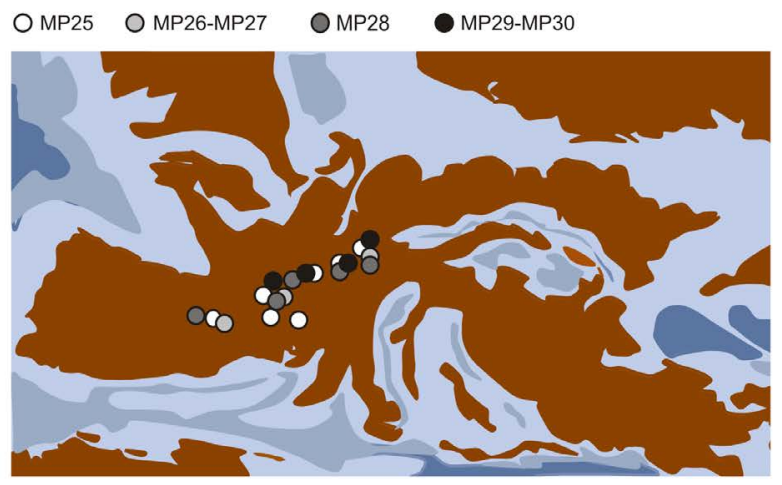

Fond paléogéographique du Chattien supérieur ( 25 m.a.)

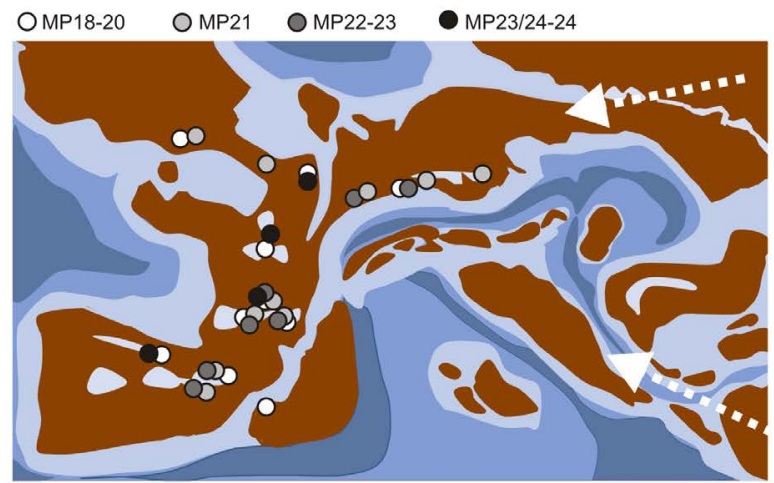

Fond paléogéographique du Rupélien inférieur (34 à 31 m.a.)
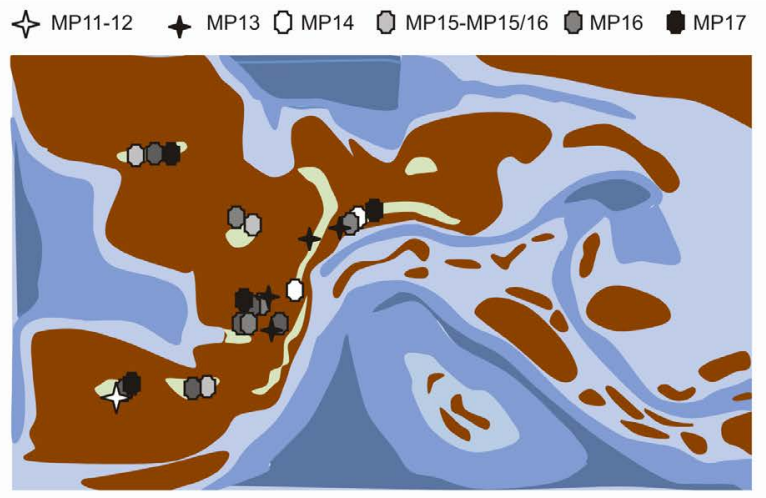

Fond paléogéographique Bartonien-Priabonien (41 à 35 m.a).

Figure 2. Localisation des principales localités fossilifères ayant livré des Theridomorpha (regroupées par MP $=$ niveaux repères mammaliens $d u$ Paléogène, cf. Tabl. 2) sur trois fonds paléogéographiques successifs. Les contours sont tirés de Kócsis et al. (2014), Schettino \& Turco (2011) et Picot et al. (2008). Les mers sont en dégradés de bleu, le plus foncé étant le plus profond ; les terres sont de couleur marron et portent des étendues aquatiques vert-pâle pour le Bartonien - Priabonien, gris pâle pour le Rupélien inférieur. Les flèches blanches sur la paléogéographie du Rupélien inférieur correspondent aux deux voies d'immigrations possibles au moment de la " Grande Coupure ». 
Pairomys, Frontyanamys et Zamoramys, souvent considérées comme des Theridomyoidea, ont été incluses dans l'analyse. Le groupe interne comprend toutes les espèces de théridomyoïdes (Tableau 1, taxons mis en relief) depuis les plus anciennes (Eocène moyen), même si le matériel ne renseigne pas tous les loci dentaires. Quelques espèces plus récentes n'ont pas été intégrées dans l'analyse quand le nombre de loci représenté était trop limité (e.g., Blainvillimys euzetensis). Au final, le groupe interne comprend 24 genres et 110 espèces nommées, parmi lesquelles 62 ne sont pas représentées par tous les loci dentaires (Tableau 4).
Trois analyses ont été effectuées, la première étant une analyse générale (AG, pour Analyse Globale) qui comprend un maximum de taxons (132 ; voir matrice, Sup-info-1). La seconde analyse a été limitée aux taxons éocènes (AE, pour Analyse Eocène $)+$ groupe externe + groupe de branchement et quelques taxons oligocènes de Pseudosciuridae, afin de minimiser l'homoplasie récurrente. Nous avons fait de même pour les taxons oligocènes dans une troisième analyse $(\mathrm{AO}$, pour Analyse Oligocène).

La matrice a été traitée avec le logiciel NDE (Nexus Data Editor v. 0.5.0; Page, 2001) et les reconstructions

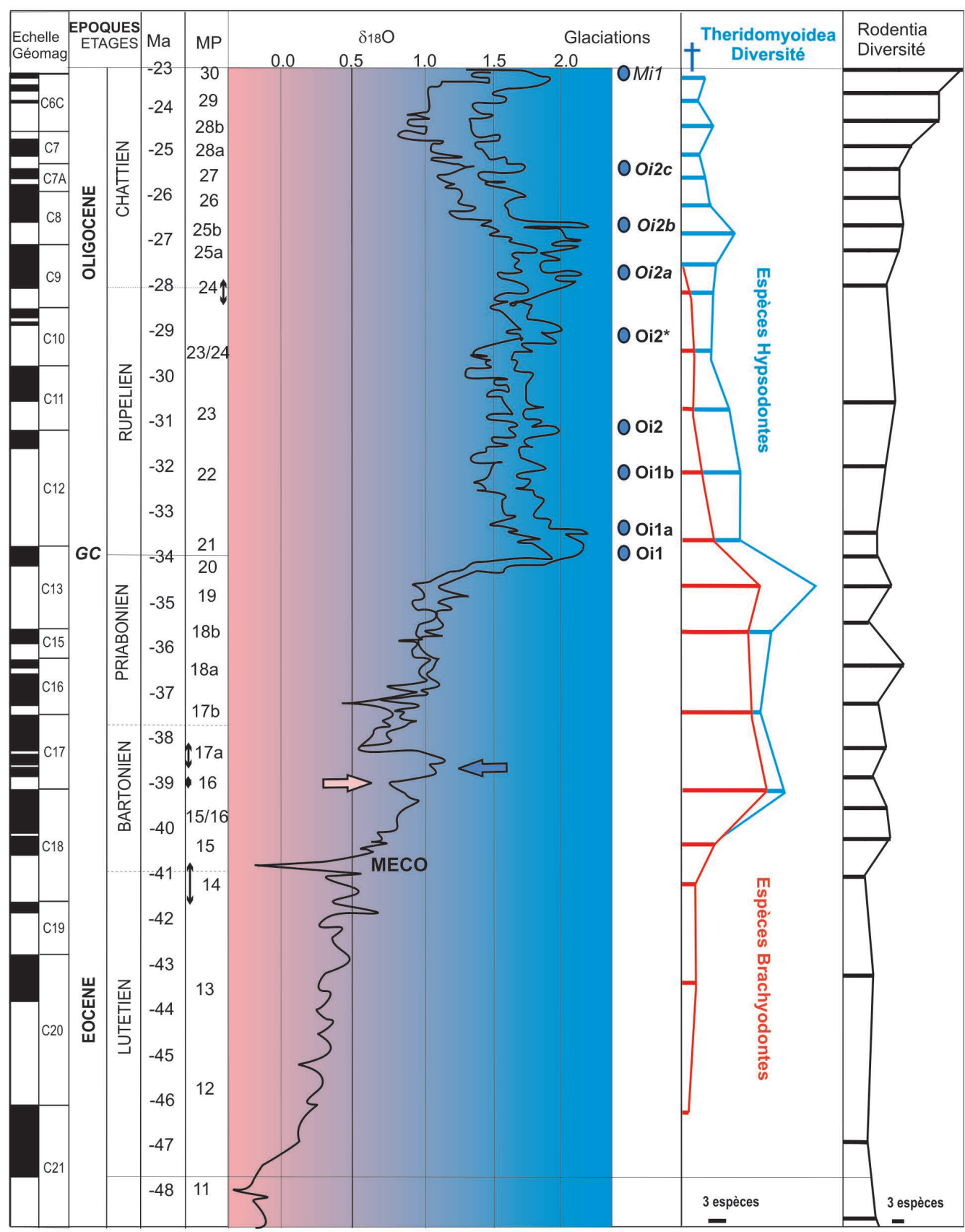

Figure 3. Situation des niveaux-repères mammaliens du Paléogène en regard de l'échelle géochronologique GTS (Gradstein et al., 2012 in Vandenberghe et al., 2012). Leur position est discutée pages 58-59. La courbe des paléotempératures et événements thermiques est schématisée à partir de Cramer et al. (2009). MECO, Middle Eocene Climatic Optimum. Oi 1 à 2c, événements froids de l'Oligocène. Mil, événement froid du Miocène basal. GC, Grande Coupure (Stehlin, 1909). Les deux colonnes de droite donnent les courbes d'évolution de la diversité des Theridomorpha (espèces brachyodontes en rouge, semi-hypsodontes, en bleu) et des rongeurs européens dans leur ensemble (en noir) : elles sont commentées pages 54-56. 
$\mathrm{S}=$ Suisse $; \mathrm{F}=$ France $; \mathrm{Es}=$ Espagne $; \mathrm{Al}=$ Allemagne $; \mathrm{An}=$ Angleterre $; \mathrm{B}=$ Belgique $; \mathrm{Cz}=$ République Tchèque

Principales localités à Theridomorpha (incl. Localités repères)

Ehrenstein 4 (Al), Coderet (F), Auvergne A.C. (Cournon ou Pérignat, Romagnat) (F), Küttigen (S)

Rickenbach, Brochene Fluh 19/20 (S), Burgmagerbein 6 (Al)

niveaux-repères

Pech du Fraysse (F), Gaimersheim 1 (Al)

Pech Desse (F), Vivel del Rio (Es), Fornant 6- 7 (S)

$\longrightarrow \quad$ MP30

Boningen 1, Wynau (S), Ehrenstein 7, Burgmagerbein 1, Gaimersheim 2 (Al), Sarèle, Boujac (F)

Mina-del-Pilar, Alcorisa (Sp)

Oensingen 11, Mümliswyl-Hardberg, Aarwangen (S), St Henri, Les Milles, Mas-de-Pauffié, St-Privatdes-Vieux, La-Devèze, Espeyrasse, St-Menoux (F), Burgmagerbein 1 (Al)

Burgmagerbein 2, Habach 4-5 (Al), Campins, Can Quarante (Es), Talent7 (S)

Antoingt et div. locs. A.C. Auvergne, Aubenas-les-Alpes, Belgarric 1, Rigal-Jouet 1, Garouillas (F),

Mas-de-Gaston (F)

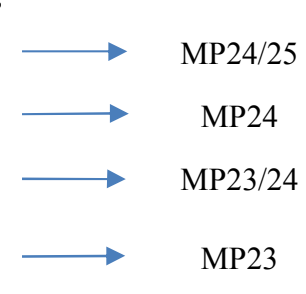

Lebratières 14, Appelle, Vialenc (F), Heimersheim (Al)

St-Martin-de-Castillon, Gas, Maintenon (F)

Lovagny, Les Chapelins, Pech Crabit, Itardies, La-Sauvetat (A.C. Auvergne) (F), Bernloch,

Schelklingen 1 (Al), Montalban 1D, Tarrega, Ciutadilla (Es),

Villebramar, Mas-de-Got A \& B, La Plante2, Cavalé, Valbro, Ruch, Pouquette, ? Armissan (F), Balm (S), Ronheim 1, Herrlingen 1, Möhren 13 (Al)

Soumailles, Ronzon, Mazan, Ravet, Aubrelong 1 (F), Hoogbutsel (B), Möhren 19-20, Ehrenstein 1 B $(\mathrm{Al})$, Detán $(\mathrm{Cz})$ Hamstead beds (An) ? Santpedor, Calaf, Aguatón, Los Barros, Villarosano 14 (Es)
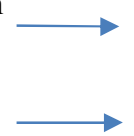

MP22

MP21

St-Capraise-d'Eymet, Tabarly, Coyrou 1 (F), Neustadt, Nordhausen (Al)

Romainville, Escamps, Baby II, Civrac, Ste-Croix-de-Brignon, Monac (F), San-Cugat-de-Gavadons, Villarosano 1 (Es), Möhren 13-19 (Al)

Lacey's farm quarry, Totland Bay, Headon Hill 2 - 4 (An), Ste-Croix-de-Beaumont (F)

La Débruge, Ste-Néboule, Lascours (F), Headon Hill 1, Whitecliff Bay 1 (An)

Gousnat (F)

Perrière, Malpérié (F), Sossis, Roc-de-Santa (Es)

Fons 4, Euzet, Aubrelong 2, La Bouffie, Les Clapies (F)

Mazateron, Minana (Es)

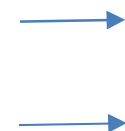

MP19-20

MP18-19
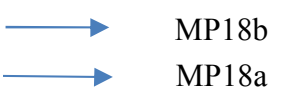

$\longrightarrow \quad \mathrm{MP} 17 \mathrm{~b}$

$\longrightarrow \quad$ MP17a

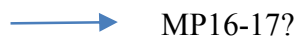

Le-Bretou, Lavergne, Robiac, Grisolles (F), Eclepens-Gare (S), Herrlingen 4 (Es), Creechbarrow (An), Babilafuente, Pontils (Es)

Les-Alleveys (S), Chéry-Chartreuve (F)

Sant-Jaume-de-Frontyana 1 (Es)

Egerkingen $\boldsymbol{\alpha}$ et $\boldsymbol{\beta}(\mathrm{S})$, Lissieu, La-Prade (F)

Bouxwiller, Le Guépelle (F)

Saint-Maximin (F)

Aumelas (F)

Casa-Ramon (Es)
MP16

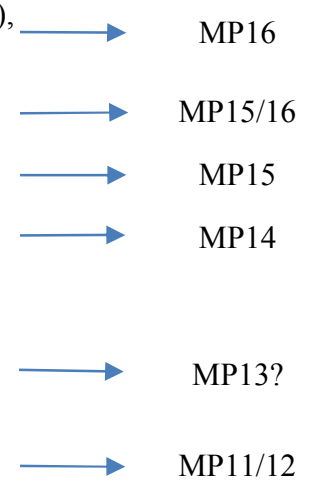

MP13?

Tableau 2. Principales localités ayant livré des Theridomorpha. Elles sont rangées face au niveau - repère duquel elles sont rapprochées (correspondance indiquée par une flèche) ; elles sont placées les unes à la suite des autres et la liste inclut la localité-type (en caractères gras). Quand des localités incluent des taxons présentant des degrés évolutifs intermédiaires entre ceux connus de deux MP successifs, leur position est notée par «MPx/ $\mathrm{x}+1 »$. Lorsque le choix de corrélation est incertain entre deux MP, les localités sont notées «MPx- $\mathrm{x}+1 »$. 
phylogénétiques exécutées avec PAUP* v.4.0 beta 10 Win (Swofford, 2002). Etant donné que la matrice contenait un grand nombre de taxons, une recherche exhaustive des arbres les plus parcimonieux n'a pas été possible (option de recherche de type «Branch and Bound » [option BandB]). Nous avons donc privilégié la méthode de recherche heuristique (option Hsearch) en la répliquant 1000 fois et en imposant une addition aléatoire de séquences taxonomiques à chaque replication. Pour les mêmes raisons (taille de la matrice à traiter), la robustesse des nœuds de l'Analyse générale (AG) a été évaluée en calculant le pourcentage de « Bootstrap » $(\mathrm{BP})$ à chaque nœud.

Description de quelques caractères sélectionnés. Afin de faciliter la lecture et la compréhension du texte, seuls quelquesuns des 315 caractères sont détaillés ci-après. Il s'agit, pour la plupart d'entre eux, de préciser des éléments de la terminologie dentaire de la figure 4. Les autres caractères sont listés en informations supplémentaires (Sup-info-2).

Hypsodontie. Le caractère 6 décrit le degré d'hypsodontie des molaires supérieures : on observe en effet des différences de la hauteur des couronnes dentaires des différentes espèces de mêmes genres.

Différenciation de l'épaisseur de l'émail sur les flancs opposés des lophes et des lophides, sinus et sinuside (Car. 7). Ce caractère a été utilisé notamment pour distinguer les espèces oligocènes des genres Blainvillimys et Theridomys (e.g., Vianey-Liaud, 1972a, 1979, 1989, 1998 ; Vianey-Liaud \& Ringeade, 1993 ; Comte et al., 2012).

Antérostyle (car. 16-17, 66-67, 125-126). Ce petit tubercule est placé mésialement et un peu lingualement au paraconule, comme le préprotoconule d'Archetypomys (Meng et al., 2007). Il peut être présent même si le paraconule est absent, à la jonction de l'antérolophe et du bras antérieur du protocône (preprotocrista).

La forme des conules (car. 26, 43, 78, 95, 134, 156), cônes (car. 27, 40, 79, 90, 108, 135, 153, 177) et conides (car. 231, 235,237 ) est ici plus détaillée qu'usuellement, afin de prendre en compte toute la diversité de leurs forme et position au sein des théridomorphes, car on ne pouvait se limiter à indiquer qu'ils sont renflés ou fusionnés dans les lophes.

Le métalophe a plusieurs parcours possibles : vers le bras antérieur de l'hypocône, vers l'hypocône, vers le postérolophe. En fait chez les théridomorphes, il peut exister deux lophules issus du métacône et se dirigeant lingualement : le métalophule I est le plus mésial (car. 45-46, 96-97, 158-160), le métalophule II est le plus distal (car. 47, 98-99, 161-163). Les deux peuvent coexister ou bien seul le métalophule II est présent.

L'endolophe - connexion mésio-distale la plus linguale entre le protocône et l'hypocône - est décomposé entre ses différents éléments (car. 52, 110, 180). La même chose est faite pour la mure - connexion mésio-distale plus buccale que l'endolophe (car. 54-55, 113, 183).

Nous utilisons la terminologie de Stehlin \& Schaub (1951) pour les vallées des dents. Ento- et ectoflexus sont nommés respectivement sinus (car. 56, 115) et synclinaux, numérotés pour ces derniers de «SI $»$ à « SIV » (car. 68, 100-103, 164-172), avec les synclinaux surnuméraires « $\mathrm{S} 0$ », mésial, ou « SV », distal (car. 60, 104, 173) si nécessaire. Ecto et entoflexides sont respectivement nommés sinuside (car. 273) et synclinides, numérotés de «sI » à «sIV » (car. 216-218, 220-227, 282), avec le synclinide surnuméraire (pré-antérosynclinide) « s0 » éventuellement (car. 227-228) (Fig. 4).

Un ectocingulum bas (car. 151-152) peut se développer mésialement et distalement à partir du mésostyle sur les flancs buccaux du paracône et du métacône, distinct des para- et méta- cristae.

Une définition plus précise du métalophide des molaires inférieures s'est avérée nécessaire. Classiquement et encore dans Marivaux et al. (2004), le métalophulide I est la connexion la plus mésiale entre le protoconide et le bras antérieur du métaconide, tandis que le métalophulide II est la connexion la plus distale (= protolophide), entre le bras postérieur du protoconide et le métaconide. Or, les attaches buccales du métalophide ne se limitent pas à ces deux positions. De ce fait, nous avons décomposé ce caractère pour décrire trois modes d'attache buccale : antérieure au sommet du protoconide, exactement au sommet du protoconide, ou enfin postérieure au sommet (car. 240-246). Dans les cas de dp4s très étirées antérieurement (i.e., Issiodoromys de l'Oligocène supérieur), il est difficile de distinguer ce qui est homologue du métaconide et du métalophide, ou s'il s'agit de néo-métalophide (car. 240-241) (Figs. 5 et 6).

A

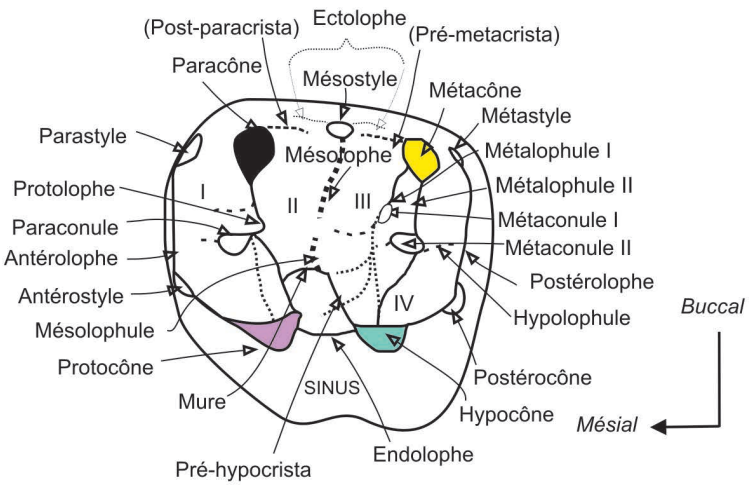

Différent parcours du protolophe et du métalophule

Crestules ..... II, II, III, IV = synclinaux

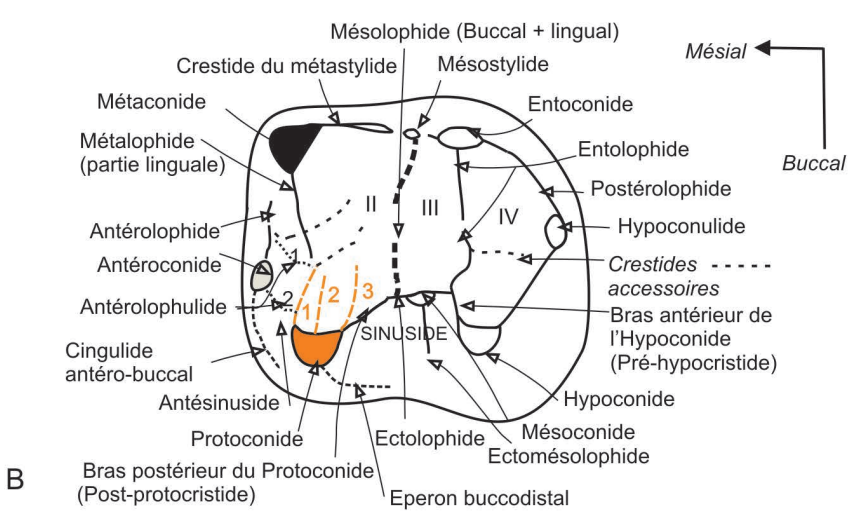

Différentes positions de l'antérolophulide 1 et 2........... I, II, III, IV = synclinides Différentes positions de la partie buccale du métalophide 1, 2, 3 -_-_-_---

Figure 4. Terminologie dentaire utilisée. A. dents jugales supérieures, B. dents jugales inférieures. En partie d'après Wood \& Wilson (1936), Stehlin \& Schaub (1951), Marivaux et al. (2004), Comte et al. (2012). Pour faciliter la lecture des figures suivantes, les quatre cuspides principales ont été colorées : en noir plein pour le paracône, en jaune pour le métacône, en violet pour le protocône, en vert pour l'hypocône. Les autres cuspides sont en formes vides (en blanc). Aux dents inférieures, c'est le métaconide qui est figuré en noir, pour le distinguer des autres cuspides linguales (non colorées) ; le protoconide est de couleur vive (orangé) afin de bien le distinguer à la fois du métaconide mais aussi de cuspides pré-protoconide (en gris). 
L'ectolophide demande une définition précise, car ce lophide mésio-distal - ou oblique - (portant éventuellement un mésoconide) a souvent été mal identifié (e.g., Hooker \& Weidmann, 2000). Il doit être distingué de la postprotocristide (= bras postérieur du protoconide) et de la préhypocristide (= bras antérieur de l'hypoconide) (car. 252-256).

L'entolophide désigne ici l'hypolophide de Wood et Wilson (car. 291-298), car ce lophide se développe à partir de l'entoconide. Il est libre buccalement pour les espèces chez qui il est bas et incomplet. Ensuite, lorsqu'il est long et complet, il se connecte généralement à l'extrémité linguale de la préhypocristide ou à l'ectolophide, mais pas directement à l'hypoconide. Ainsi, le terme entolophide décrit mieux l'origine et la topographie de ce lophide non seulement pour les Theridomopha, mais pour l'ensemble des rongeurs.

\section{Résultats}

Les analyses heuristiques effectuées sur le jeu de données taxons/ caractères total $(\mathrm{AG})$ ont généré trois arbres équiparcimonieux de 2205 pas chacun (Indice de cohérence [Ic] $=0.196$; Indice de Rétention $[\mathrm{Ir}]=0.762)$. Un arbre de consensus strict est présenté en figures 7A et 7B. Les analyses partielles réalisées d'une part sur les genres éocènes (AE) et d'autres part sur les genres oligocènes $(\mathrm{AO})$ ont respectivement fourni huit arbres équiparcimonieux de 1725 pas chacun $(\mathrm{Ic}=0.225 ; \mathrm{Ir}=0.666)$ et deux arbres équiparcimonieux de 1359 pas chacun (Ic = 0.296 ; Ir $=0.752$ ). Pour ces deux analyses partielles d'un point de vue taxonomique, des arbres de consensus strict sont présentés en figure 8 (AE) et figure 9 (AO).

AG (Analyse Globale de tous les taxons). La liste des caractères aux nœuds est donnée in extenso en informations supplémentaires (Sup-info. 3). En présentant la topologie de l'arbre de consensus strict, nous discuterons les caractères principaux qui soutiennent les 23 nœuds de cet arbre (nœuds
$A$ à $W$ ). Les caractères non ambigus sont notés NA et les caractères ambigus, A. Les pourcentages de Boostrap (BP) sont indiqués sur les figures 7A et 7B et rappelés ci-dessous. Le cas échéant, lorsque des différences apparaîtront entre la topologie $\mathrm{AG}$ et les topologies des analyses partielles $\mathrm{AE}$ ou $\mathrm{AO}$, nous les discuterons à ce moment.

- La racine de l'arbre est identifiée au noud $A$ ( 25 caractères NA et $9 \mathrm{~A})$, par les Glires archaïques + Cocomys $(\mathrm{BP}=69)$ et Tanquammys. Parmi les caractères, on notera la DP4 avec un métalophule I en deux parties (endolophe présent et sinus court seulement chez Tanquammys) et l'absence du métacône sur P4. L'incisive est longue (extrémité distale en arrière de m3), plus courte chez Tanquammys (sous m3), l'antérolophide des molaires est absent, l'hypoconide est absent sur $\mathrm{p} 4$, mais présent et étendu buccalement sur les molaires, leur mésostylide est absent, le métaconide est beaucoup plus élevé que l'entoconide et l'entolophide est incomplet (sauf chez Tanquammys).

- Le noud B (19 caractères NA et $3 \mathrm{~A})$, robustesse (BP = 83), exprime la proximité phylogénétique de Euromys (considéré comme un Ailuravinae ; e.g., Escarguel, 1999) avec les reithroparamyinés ischyromyoïdes. Euromys montre un ensemble de caractères qui apparaissent primitifs dans cette analyse (DP4 avec protolophe incomplet, alors qu'il est complet chez les autres [i.e., Ischyromyoidea + Theridomorpha]; M3 plus longue que la M2, alors qu'elle est de longueur égale chez les autres, hypocône lingual au protocône ; sur les m1-2s : antéroconide présent, ectolophide absent, mésostylide indistinct, hypoconulide isolé) et d'autres spécialisés (sommet du protocône aigu, base légèrement étirée mésio-distalement ; long ectocingulum présent, métaconule égal au protoconule, bassin du talonide rugueux à ridulé).

Sur l'arbre de consensus strict (Fig. 7A), deux groupes se détachent ensuite successivement, d'abord un clade correspondant aux Reithroparamyinae nord-américains

\begin{tabular}{|c|c|c|c|}
\hline \multicolumn{4}{|c|}{ Groupes externe et de branchement } \\
\hline GLIRES, RODENTIAFORMES & & Age & Observations \\
\hline Alagomyidae & Dasheveg, 1990 & & \\
\hline Tribosphenomys minutus & Meng et al., 1994 & Paléocène terminal & Bibliographie \\
\hline Archetypomyidae & Meng et al., 2007 & & \\
\hline Archetypomys erlianensis & Meng et al., 2007 & Eocène inférieur terminal & Bibliographie \\
\hline \multicolumn{4}{|l|}{ CTENOHYSTRICA } \\
\hline Cocomyidae & Dawson et al., 1984 & & \\
\hline Cocomys lingchaensis & (Li et al., 1979) & Eocène inférieur & Moulage \\
\hline \multicolumn{4}{|l|}{ Ctenodactyloidea } \\
\hline Tanquammys wilsoni & Dawson et al., 1984 & Eocène moyen & Moulage \\
\hline \multicolumn{4}{|c|}{ ISCHYROMYIFORME } \\
\hline Ischyromyidae & Alston, 1876 & & \\
\hline Acritoparamys atavus & (Jepsen, 1937) & Paléocène supérieur & Moulage \\
\hline \multicolumn{4}{|c|}{ Sciuravinae } \\
\hline Knightomys depressus & (Loomis, 1907) & Eocène inférieur & Moulage \\
\hline Knightomys minor & Wood, 1965 & Eocène inférieur & Bibliographie \\
\hline Prolapsus sibilatoris & Wood, 1973 & Eocène moyen & Bibliographie \\
\hline Ailuravinae & Michaux, 1968 & & \\
\hline Euromys cardosoi & (Estravis, 1992) & Eocène inférieur & Bibliographie \\
\hline Pseudoparamyinae & Michaux, 1964 & & \\
\hline Plesiarctomys savagei & (Michaux, 1964) & Eocène inférieur-moyen & Original \\
\hline Pseudoparamys teilhardi & (Wood, 1962) & Eocène inférieur & Bibliographie \\
\hline Reithroparamyinae & Wood, 1962 & & \\
\hline Sparnacomys chandoni & Hartenberger, 1971 & Eocène inférieur & Moulage \\
\hline sous-famille indet. & in Escarguel, 1999 & & \\
\hline Corbarimys hottingeri & Marandat, 1989 & Eocène inférieur & Original \\
\hline
\end{tabular}

Tableau 3. Liste des taxons constituant les groupes externe et de branchement de l'analyse cladistique. 


\begin{tabular}{|c|c|c|c|c|c|c|}
\hline Taxons avec locus manquants & DP4 & P4 & M1-2 & dp4 & p4 & Total \\
\hline Archetypomys erlaniensis & & & & & & $1 / 6$ \\
\hline Cocomys lingchaensis & & & & & & $1 / 6$ \\
\hline Tanquammys wilsoni & & & & & & $1 / 6$ \\
\hline Reithroparamys delicatissimus & & & & & & $2 / 6$ \\
\hline Acritoparamys atavus & & & & & & $1 / 6$ \\
\hline Knightomys depressus / minor & & & & & & $2 / 6$ \\
\hline Prolapsus sibilatoris & & & & & & $2 / 6$ \\
\hline Euromys cardosoi & & & & & & $1 / 6$ \\
\hline Sparnacomys chandoni & & & & & & $1 / 6$ \\
\hline Zamoramys extraneus & & & & & & $3 / 6$ \\
\hline Protadelomys cartieri & & & & & & $2 / 6$ \\
\hline Protadelomys lugdunensis & & & & & & $1 / 6$ \\
\hline Tardenomys chartreuvensis & & & & & & $1 / 6$ \\
\hline Sciuroides siderolithicus & & & & & & $3 / 6$ \\
\hline Sciuroides ehrensteini & & & & & & $1 / 6$ \\
\hline Sciuroides russelli & & & & & & $1 / 6$ \\
\hline Sciuroides romani & & & & & & $1 / 6$ \\
\hline Sciuroides rissonei & & & & & & $1 / 6$ \\
\hline Treposiurus mutabilis & & & & & & $2 / 6$ \\
\hline Treposciurus intermedius & & & & & & $4 / 6$ \\
\hline Treposciurus manentis & & & & & & $3 / 6$ \\
\hline Suevosciurus minimus & & & & & & $4 / 6$ \\
\hline Suevosciurus (Escamps) & & & & & & $1 / 6$ \\
\hline Suevosciurus fraasi & & & & & & $2 / 6$ \\
\hline Suevosciurus bosmae & & & & & & $2 / 6$ \\
\hline Tarnomys helveticus & & & & & & $2 / 6$ \\
\hline Tarnomys quercinus & & & & & & $2 / 6$ \\
\hline Tarnomys schmidtkittleri & & & & & & $3 / 6$ \\
\hline Tarnomys depereti & & & & & & $3 / 6$ \\
\hline Tarnomys spelaeus & & & & & & $1 / 6$ \\
\hline Pseudosciurus suevicus & & & & & & $1 / 6$ \\
\hline Estellomys cansouni & & & & & & $3 / 6$ \\
\hline Paradelomys crusafonti & & & & & & $1 / 6$ \\
\hline Columbomys cuencae & & & & & & $2 / 6$ \\
\hline Columbomys agusti & & & & & & $3 / 6$ \\
\hline Columbomys lavocati & & & & & & $2 / 6$ \\
\hline Elfomys engesseri & & & & & & $1 / 6$ \\
\hline Elfomys parvulus & & & & & & $2 / 6$ \\
\hline Elfomys hartenbergeri & & & & & & $1 / 6$ \\
\hline Issiodoromys nanus & & & & & & $1 / 6$ \\
\hline Issiodoromys pseudanaema & & & & & & $1 / 6$ \\
\hline Pseudoltinomys phosphoricus & & & & & & $1 / 6$ \\
\hline Pseudoltinomys cosetanus & & & & & & $3 / 6$ \\
\hline Pseudoltinomys amblesi & & & & & & $2 / 6$ \\
\hline Ectropomys monacensis & & & & & & $1 / 6$ \\
\hline Blainvillimys euzetensis & & & & & & $4 / 6$ \\
\hline Blainvillimys stehlini & & & & & & $1 / 6$ \\
\hline Thalerimys perrealensis & & & & & & $1 / 6$ \\
\hline Theridomys lembronicus & & & & & & $1 / 6$ \\
\hline Theridomys octogesensis & & & & & & $2 / 6$ \\
\hline Theridomys bonduelli & & & & & & $1 / 6$ \\
\hline Theridomys campinsensis & & & & & & $2 / 6$ \\
\hline Archaeomys / Blainvillimys robustus & & & & & & $2 / 6$ \\
\hline Archaeomys / Blainvillimys sp. & & & & & & $1 / 6$ \\
\hline Archaeomys / Blainvillimys huerzeleri & & & & & & $1 / 6$ \\
\hline Archaeomys helveticus & & & & & & $2 / 6$ \\
\hline Archaeomys arvernensis & & & & & & $2 / 6$ \\
\hline Remys garimondi & & & & & & $4 / 6$ \\
\hline Remys major & & & & & & $5 / 6$ \\
\hline Pairomys ibericus & & & & & & $3 / 6$ \\
\hline Pairomys crusafonti & & & & & & $4 / 6$ \\
\hline Bernardia marandati & & & & & & $4 / 6$ \\
\hline
\end{tabular}

Tableau 4. Liste des taxons avec loci dentaires manquants (cellules grisées). 
(incluant Reithroparamys delicatissimus et Acritoparamys atavus) puis Corbarimys hottingeri, européen, de position systématique indéterminée. Escarguel (1999 : 256) notait les ressemblances entre ce genre et Zamoramys : il apparaît ici comme un taxon basal proche de l'ensemble Sciuravidae + Remyoidea.

- Le nøud $C$ exprime la position basale du groupe Sciuravidae + Remyidae au sein des Ischyromyiformes. Ce nœud $\mathrm{C}$ est soutenu par 20 caractères NA et six A $(B P=37)$. Parmi les caractères non ambigus, notons la forme du protocône des DP $4 \mathrm{~s}$, bulbeuse chez les premiers, tandis qu'elle est saillante chez les Ischyromyidae, alors que l'hypocône des molaires est bulbeux chez les Ischyromyidae et légèrement étiré mésio-distalement chez les Remyoidea + Sciuravidae. Le protolophe est connecté à la preprotocrista sur les molaires des Ischyromyidae, au protocône chez les Remyoidea + Sciuravidae ; le mésostyle est plus buccal chez les Ischyromyidae, alors qu'il est proche de

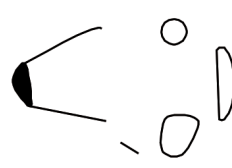

Remys minimus

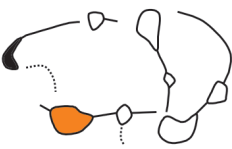

Treposciurus gardneri

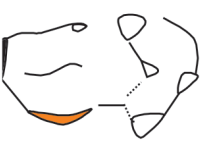

Elfomys catalaunicus

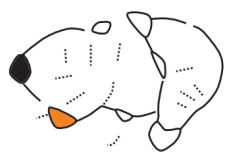

Sciuroides russelli

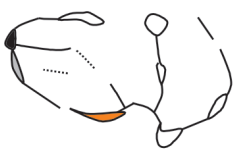

Elfomys tobieni dp4s inférieures

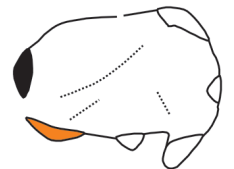

Hartenbergeromys hautefeuille

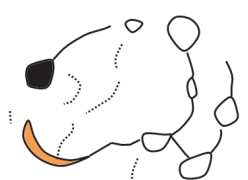

Pantrogna marandati
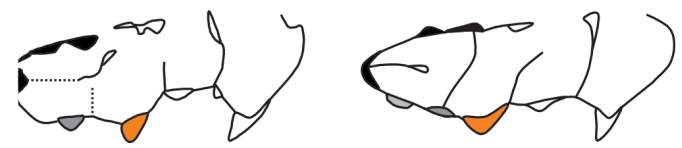

Issiodoromys pauffiensis

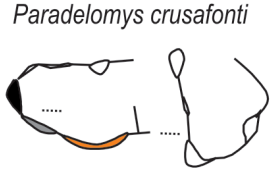

Issiodoromys hartenbergeri

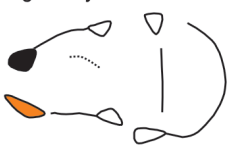

Protadelomys maximini

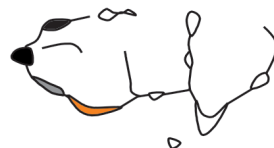

Issiodoromys medius

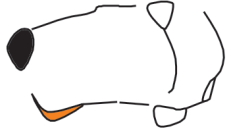

Protadelomys nievesae

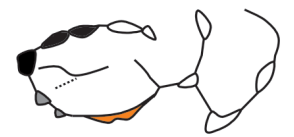

Issiodoromys minor

ssiodoromys quercyi

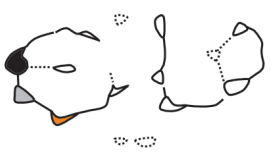

Pseudoltinomys cuvier

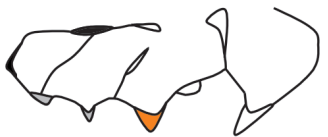

Issiodoromys limognensis

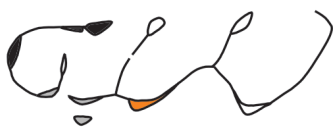

Issiodoromys bransatensis

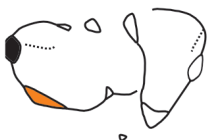

'seudoltinomys crebrum

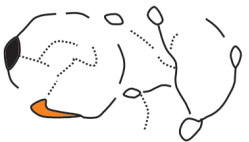

Estellomys varleti

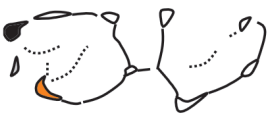

....

Datriotheridomys sudrei

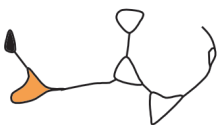

Sciuromys cayluxi

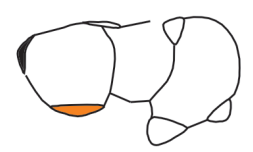

Ectropomys exiguus

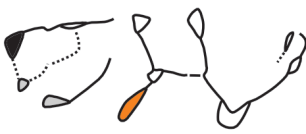

Patriotheridomys neboulensis

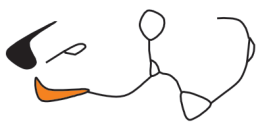

Sciuromys rigali

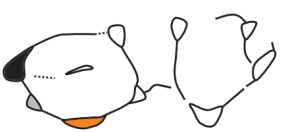

Pseudoltinomys gaillardi

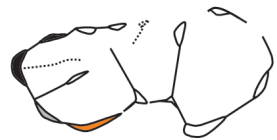

Pseudoltiñomys major

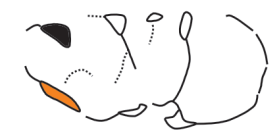

Oltinomys platyceps

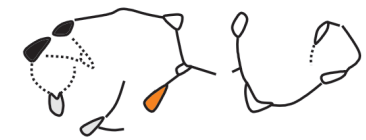

Patriotheridomys altus

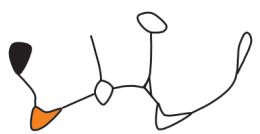

Columbomys lavocati

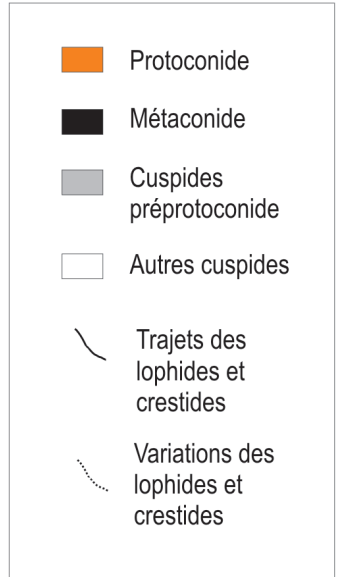

Trajets des lophides et

Variations des

Figure 5. Schémas des dp4s inférieures de diverses espèces de Remyoidea et Theridomorpha : Theridomyoidea indet., divers Pseudosciuridae, divers Theridomyidae (indet., Issiodoromyinae, Oltinomyinae, Patriotheridomyinae et Columbomyinae). Les noms des espèces sont indiqués au dessous de chaque schéma. Il s'agit dans la plupart des cas de schémas réalisés à partir de spécimens de la population type, de moulages ou de figurations originales. Les cuspides sont schématisées par le contour de l'émail qui les borde, pris sur des dents peu usées, en noir plein pour le métaconide et ses possibles dérivés, en orange pour le protoconide et en gris pour les cuspides supplémentaires mésiales au protoconide, en formes vides pour les autres cuspides. Les trajets des crestides et lophides sont schématisés par de simples traits pleins. Leurs variations et celles des crestules accessoires sont en traits pointillés. Ces trajets peuvent être reconnus par comparaison avec la figure 4 (terminologie). Ces conventions de figuration seront les mêmes pour les figures 6, 10 à 12, 14 . 
l'alignement du paracône et du métacône ; le métalophule I est présent chez les Ischyromyidae, absent chez les Remyoidea + Sciuravidae chez qui seul le métalophule II est présent (mais seulement chez les Sciuravidae). L'endolophe des P4s est bas chez les Ischyromyidae, élevé chez les autres (absent chez les Sciuravidae). Ainsi, dans cette analyse, les Sciuravidae sont le groupe-frère des Remyidae, avec lesquels ils constituent un clade (nœud D), alors que dans l'analyse de Marivaux et al. (2004), le seul remyoïde considéré, Zamoramys, était groupe frère d'Ailuravus, l'ensemble étant groupe frère d'Acritoparamys. Les Sciuravidae étaient membres de l'autre clade («mouse-related clade ») au sein des Ischyromyiformes. Comme aucun des genres de cet autre clade n'a été introduit dans la présente analyse, cette topologie doit être considérée avec précaution et sa robustesse relativisée.
- Noud D entre Sciuravidae et Remyidae : (27 caractères NA $+2 \mathrm{~A})$. Ce nœud est moins robuste $(\mathrm{BP}=17)$. Les Remyidae $(\mathrm{BP}=86)$ se distinguent du clade des Sciuravidae considérés notamment par : la position plus linguale de l'hypocône, la présence de l'endolophe, l'absence ou la réduction du métalophule II, l'incisive plus longue, l'attache du métalophide plus antérieure (au sommet du protoconide), la postprotocristide oblique et longue, l'attache plus antérieure de l'entolophide (à l'extrémité antérieure de la préhypocristide) et l'absence de postérolophide.

.) Le noeud D1 (24 caractères NA et $8 \mathrm{~A}$ ) exprime la proximité phylogénétique de Zamoramys avec Frontyanamys $(\mathrm{BP}=21)$ et le noud D2 (28 caractères NA et $12 \mathrm{~A})$, le lien de parenté étroit $(\mathrm{BP}=57)$ de Frontyanamys avec les

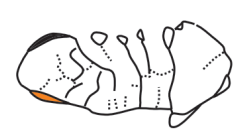

Thalerimys fordi

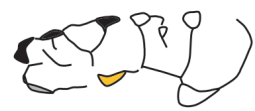

Theridomys sp.(Ruch \& Pouquette)

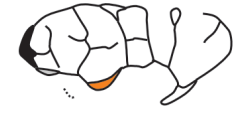

"Theridomys" bonduelli

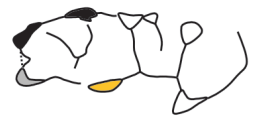

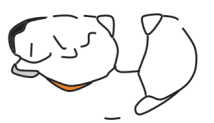

"Theridomys" golpei

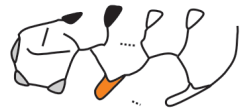

Theridomys lembronicus

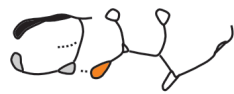

"Theridomys" aquatilis

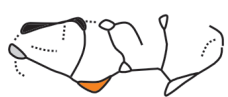

Paratheridomys margaritae Paratheridomys ludensis

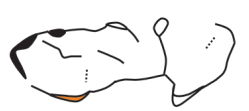

"Blainvillimys" gousnatensis

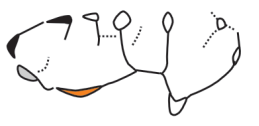

"BI." pseudosiderolithicus

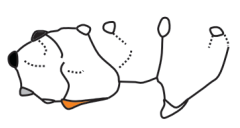

"BI." civracensis

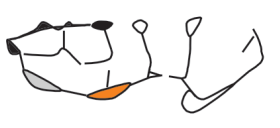

"BI." rotundidens

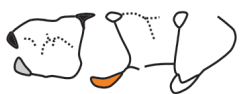

"Theridomys" calafensis
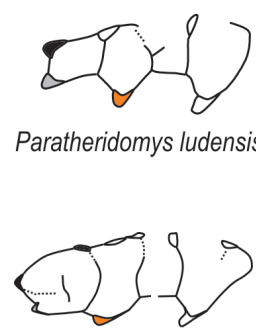

"Bl." langei

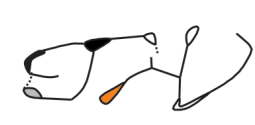

Blainvillimys gregarius

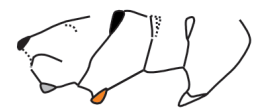

Blainvillimys gemellus

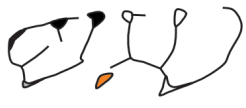

Blainvillimys heimersheimensis

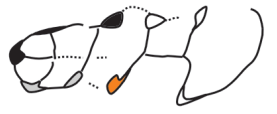

Blainvillimys helmeri

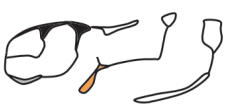

Toeniodus hexalophodus

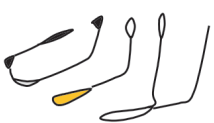

Protechimys major

Protechimys truci

Protechimys lebratierensis

Protechimys gracilis

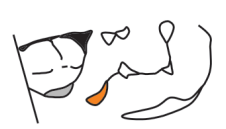

Toeniodus curvistriatus

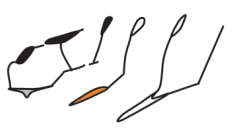

Protechimys blainvillei

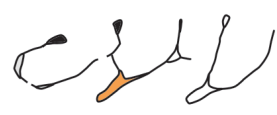

Archaeomys / Blainvillimys sp.

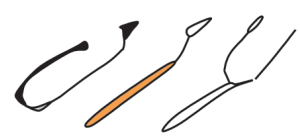

Archaeomys quercyi

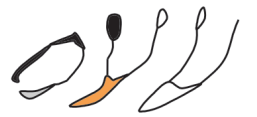

A. /B. ehrensteini

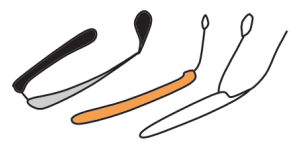

Archaeomys intermedius

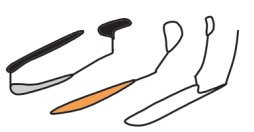

dp4s inférieures

A. /B. huerzeleri

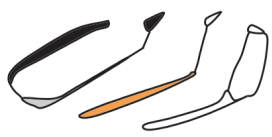

Archaeomys? helveticus

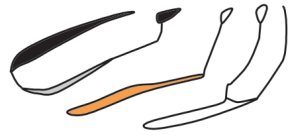

Archaeomys laurillardi

Figure 6. Schémas des dp4s inférieures de diverses espèces de Theridomyinae. Les structures impliquées dans l'allongement antérieur de ces dents diffèrent selon les genres et selon les espèces au sein des genres. Ruptures des liaisons mésio-distales, obliquité et étirement des protoconide et hypoconide se développent chez les Archaeomyini et Toniodus. 
Remyidae (clade associant Remys + Pairomys ; $\mathrm{BP}=55$ ). Zamoramys montre un ensemble de caractères qui peuvent être considérés comme primitifs pour les remyoïdes : les dents sont brachyodontes, les styles (para- et méso-) sont présents et forts, tandis que l'antérostyle est absent ; le métalophule II est absent, l'hypocône est lingual, bas et plus petit que le protocône ; les $\mathrm{m} 3 \mathrm{~s}$ sont plus longues que les $\mathrm{m} 2 \mathrm{~s}$, l'antérolophide et l'antésinuside sont présents sur les $\mathrm{m} 1-2 \mathrm{~s}$; le protoconide est bulbeux, la branche buccale du métalophide se connecte au sommet du protoconide, la postprotocristide est oblique et moins longue que celle de Frontyanamys, le mésoconide est présent et l'hypoconulide est isolé et fort.

.) Au niveau du noeud D2, l'hypocône est aussi haut que le protocône ; il demeure plus petit et bulbeux chez Frontyanamys et devient aussi long et crescentiforme dans le clade des Remyidae ; 1'endolophe demeure présent chez Frontyanamys et peut disparaître chez les Remyidae ; les antérolophide, antésinuside et mésostylide disparaissent chez ces derniers, tout comme l'ectolophide, tandis que l'entolophide s'oriente vers l'arrière et s'interrompt ; l'hypoconulide s'étire dans le postérolophide.

- Le pendant du clade caractérisé en D est celui du nœud $E$, relativement peu robuste $(\mathrm{BP}=13)$, qui sépare au sein des autres Ischyromyiformes un clade $(\mathrm{BP}=46)$ correspondant à deux genres de Pseudoparamyinae européens (Plesiarctomys et Pseudoparamys) de Sparnacomys, un Reithroparamyinae européen. Ce nœud est soutenu par 16 caractères NA et sept A. Parmi eux on peut noter l'endolophe « vrai » et bas sur les DP4s des pseudoparamyinés (tandis qu'il est constitué par des éperons obliques issus des flancs opposés du protocône et de l'hypocône chez Sparnacomys), le métalophule I plus développé sur les M1-2s de Sparnacomys, l'antéroconide, l'antésinuside et l'entolophide présents chez Sparnacomys alors qu'ils sont absents chez les pseudoparamyinés ; le protoconide pointu chez Sparnacomys, tandis qu'il est bulbeux chez les Plesiarctomys et Pseudoparamys.

Sur l'arbre de consensus strict (Fig. 7A), toute une série de groupes se détachent ensuite successivement, dont les deux premiers nœuds sont analysés ici, les nœuds $\mathrm{F}(\mathrm{BP}=21)$ et $\mathrm{G}$ $(\mathrm{BP}=54), \mathrm{F}$ montrant l'étroite parenté de Sparnacomys avec le clade P-H (Pantrogna - Hartenbergeromys). Ces trois genres sont considérés comme des Reithroparamyinae européens (cf. Escarguel, 1999), alors que selon nos résultats, les reithroparamyinés typiques (nord-américains) sont nettement plus basaux. Le nœud $\mathrm{G}$ exprime la proximité phylogénétique des représentants du clade $\mathrm{P}-\mathrm{H}$ avec les premiers Protadelomys. Pantrogna et Hartenbergeromys représentent les genres basaux de l'ensemble Theridomorpha.

- Le noud $F$ est soutenu par 20 caractères NA et un A. Parmi eux, les Theridomorpha se distinguent de Sparnacomys par la position moins buccale du mésostyle sur les dents supérieures, le mésolophe présent et connecté à des éperons et crestules, le métaconule II petit, l'hypocône aussi haut que le protocône bien que moins volumineux, la $\mathrm{m} 1$ plus courte que la $\mathrm{m} 2$, les métaconide et protoconide peu rapprochés sur les $\mathrm{p} 4 \mathrm{~s}$ et bien distincts sur les $\mathrm{dp} 4 \mathrm{~s}$. Aux dents inférieures, les caractères comme la présence d'une longue et oblique postprotocristide, un ectolophide court, la présence d'un entolophide, l'hypoconulide réduit ou absent et étiré dans le postérolophide, constituent les apomorphies de l'ensemble des Theridomorpha (e.g., Figs. 5-6 et 10).
Sur l'arbre de consensus strict (Fig. 7A), quatre ensembles se détachent successivement, dont les premier et dernier nœuds sont analysés ici, les nœuds $\mathrm{G}$ et $\mathrm{H}$ : le premier $(\mathrm{G})$, modérément robuste $(\mathrm{BP}=54)$, traduit la proximité phylogénétique du clade P-H (Pantrogna - Hartenbergeromys) avec Protadelomys cartieri et ensuite, successivement, la proximité avec les autres espèces de Protadelomys : P. lugdunensis $(\mathrm{BP}=30)$ et $P$. alsaticus $(\mathrm{BP}=25)$, puis $(\mathrm{BP}=28) P$. maximini et $P$. nievesae; ces derniers constituent un sous-clade $(\mathrm{BP}=65)$. Le nœud H $(\mathrm{BP}=31)$ indique l'étroite parenté de Protadelomys avec Tardenomys chartreuvensis et le large clade J. Le genre Protadelomys apparaît ici paraphylétique, mais cette topologie pourrait correspondre à la représentation de grades évolutifs au sein d'un même genre. Il ne serait pas raisonnable de donner à chacun un nom de genre différent, ce qui complexifierait inutilement la taxonomie. Il est à noter que la succession de ces branches de Protadelomys est contraire à l'ordre stratigraphique supposé, car $P$. maximini et $P$. nievesae sont considérées comme les plus anciennes espèces du genre (e.g., Escarguel, 1998). Pour mieux comprendre les relations entre ces espèces, il faudrait que chacune soit bien définie, sa variabilité bien connue, ce qui n'est pas toujours le cas (cf. p. 49).

- Le næud $G$ est soutenu par 28 caractères NA et six A. Parmi eux, le métacône renflé montre un sommet saillant et aigu, tandis que l'hypocône est étiré mésio-distalement sur les $\mathrm{P} 4 \mathrm{~s}$; le paraconule est proéminent et étiré mésio-distalement ; l'endolophe est élevé ; le métalophule II est présent sur les DP4s et les molaires et situé à côté du métalophule I bas et discontinu ; la longueur des $\mathrm{m} 3 \mathrm{~s}$ est réduite par rapport aux $\mathrm{m} 2 \mathrm{~s}$ ; l'ectolophide est mésio-distal ; le mésolophide est absent ; l'entolophide est constitué de deux parties non continues.

Tous les taxons successivement arrangés du nœud $\mathrm{G}$ au nœud $\mathrm{H}$ sont des Theridomorpha incertae sedis : il s'agit de Pantrogna, Hartenbergeromys, tous les Protadelomys et Tardenomys.

- Le nœud $H$ est soutenu par 29 caractères NA et deux A. Parmi eux, chez Tardenomys : sur les DP4s, le métalophule I est absent, l'hypocône est aussi haut et fort que le protocône, l'endolophe est plus bas que le protocône et le sinus atteint la partie moyenne de la dent. Sur les P4s, le SI est toujours présent, le protolophe s'attache sur le protocône, le métacône est aussi fort que le protocône, l'endolophe résulte d'éperons obliques plus ou moins développés. Les M3s sont plus petites que les M2s, le mésolophe et le mésolophule sont présents et séparés, le métaconule est réduit, le métalophule I est absent, la mure est ébauchée, le sinus est court et l'ectocingulum est présent. La $\mathrm{m} 3$ est plus longue que la $\mathrm{m} 2$ (réversion), le mésoconide est peu ou non saillant et l'ectolophide est oblique, l'entolophide est complet sur toutes les dents inférieures. Sur AE (Fig. 8), Tardenomys se retrouve après la dichotomie Pseudosciuridae Theridomyidae, en genre frère de Paradelomys.

- Le noud I (BP = 47) sépare Tardenomys du clade Theridomyoidea (nœud $\mathrm{J}$ ) ; il est soutenu par 32 caractères NA et cinq A. Parmi eux, le protolophe des DP4s et P4s, qui était incomplet chez Tardenomys est complet chez les théridomyoïdes. L'endolophe est « vrai » et le sinus court (caractères apparaissant comme des réversions à l'issue de l'analyse) et le postérolophe est connecté à l'hypocône. Sur toutes les dents supérieures, le parastyle est fort, le paraconule est présent, la mure est présente et apparaît comme une crête 
mésio-distale entre le protolophe (ou entre l'extrémité buccale de la postprotocrista) et l'extrémité buccale de la préhypocrista, l'ectocingulum est variablement présent, l'hypocône est aussi haut et fort que le protocône et le métaconule II est bulbeux. Sur les dents inférieures, l'antéroconide est fort, l'ectolophide est mésio-distal, le mésolophide est absent, l'hypoconulide est étiré dans le postérolophide, le bassin du talonide est rugueux à ridulé. Sur les p4s, l'hypoconide est aussi développé que le protoconide et l'hypoconulide est absent.

- Le næud $J(\mathrm{BP}=47)(29$ caractères NA et 9 A) illustre le clade Theridomyoidea, un clade qui se caractérise par une dichotomie principale correspondant aux deux familles : Pseudosciuridae (Pseu.) et Theridomyidae (Th.). Parmi les caractères en soutien, sur les $\mathrm{P} 4 \mathrm{~s}$, on note la connexion linguale du mésolophe avec l'extrémité buccale de la préhypocrista chez les Theridomyidae, alors qu'il est libre chez Pseudosciuridae. Sur les P4s et M1-2s, le métacône est étiré dans le métalophe, mais toujours proéminent (Th.), alors que le paracône et le métacône ont leur sommet saillant et pointu chez les Pseudosciuridae ; le métalophule I variablement présent, est anguleux (Th.) ou rectiligne et régulier (Pseu.). Sur les dp4s, le protoconide est crestiforme (Th.) ou bien bulbeux, au sommet plus ou moins pointu (Pseu.). Le métaconide est beaucoup plus élevé que l'entoconide (Pseu.) ou presque de même hauteur (Th.). L'ectolophide est séparé de la préhypocristide (Th.) ou connecté à la postprotocristide et à la préhypocristide (Pseu.) ; il sépare complètement le sinuside des synclinides opposés (Pseu.) alors qu'il laisse une communication peu profonde chez les Theridomyidae. Le mésoconide est saillant (Pseu.) ou non (Th.). Les crestides et ridules sont fréquentes (Pseu.) ou rares (Th.).

- Au noud $K$, le clade Pseudosciuridae montre un pourcentage de Bootstrap élevé $(B P=83)$, cependant les relations inter-génériques en son sein sont peu évidentes. Sur l'arbre de consensus strict d'AG (Fig. 7A), Tarnomys depereti apparaît comme le taxon le plus basal des Pseudosciuridae. Cependant, le matériel documentant ce taxon est limité au type (p4-m3) et le nœud correspondant est soutenu par seulement huit caractères NA (et $12 \mathrm{~A}$ ). Sur cette topologie, le nœud suivant place Tarnomys helveticus comme groupe frère de l'ensemble des autres Pseudosciuridae, avec 13 caractères NA et six A. Ainsi le genre Tarnomys apparaît paraphylétique. La topologie est différente sur l'analyse AE limitée au taxons éocènes (+ quelques taxons oligocènes). Sur AE (Fig. 8), deux clades de Pseudosciuridae apparaissent, le clade Tarnomys - Pseudosciurus $(\mathrm{BP}=22)$ et le clade comprenant les trois autres genres. Ces derniers s'organisent avec d'une part Sciuroides $(\mathrm{BP}=30)$ et d'autre part un sous-clade $(\mathrm{BP}=65)$ comprenant Treposciurus $(\mathrm{BP}=43)$ et Suevosciurus $(\mathrm{BP}=73)$. Tarnomys helveticus apparaît comme l'espèce sœur des autres Tarnomys + Pseudosciurus, alors que c'est « Treposciurus » manentis qui occupe cette position sur AG (Fig. 7A). Les relations entre Suevosciurus et Treposciurus sont les mieux soutenues, notamment sur AG, où elles le sont par 21 NA et 5 A. L'attribution de l'espèce manentis à Treposciurus est donc peut-être à reconsidérer.

- Le nœud L ( $\mathrm{BP}=47)$ (39 caractères NA et $7 \mathrm{~A})$ traduit la proximité phylogénétique du clade Paradelomys avec les autres Theridomyidae éocènes (Fig. 7B). Paradelomys présente des caractères des dents supérieures qui se retrouvent par convergence à des nœuds plus terminaux au sein du clade des Theridomyidae, surtout chez les formes oligocènes, mais pas chez les espèces basales. Il s'agit du protolophe complet sur les DP4s et connecté à la postprotocrista, de la mure ébauchée sur $\mathrm{P} 4 \mathrm{~s}$, du mésostyle absent sur DP4s, mais fort sur $\mathrm{P} 4 \mathrm{~s}$, du paraconule indistinct sur $\mathrm{P} 4 \mathrm{~s}$ et du postérolophe connecté à l'hypocône. Excepté le cingulide mésio-buccal présent sur les $\mathrm{p} 4 \mathrm{~s}$, les caractères des dents inférieures sont plus conservateurs. Les innovations des Theridomyidae sont avant tout le développement d'un mésocône ou d'un néomésoconide et la réduction de la longueur de l'ectolophide.

Une série d'espèces émerge ensuite successivement, à partir du nœud L, sur le cladogramme (Fig. 7B). Les quatre premières appartiennent au genre Elfomys $(\mathrm{BP}=57$ et $\mathrm{BP}=55$ et 52 pour les deux dernières qui constituent un clade), les quatre suivantes au genre Pseudoltinomys $(\mathrm{BP}=59,41,20,32)$. Nous retrouvons ici la même configuration que précédemment pour Protadelomys. Ces deux genres apparaissent paraphylétiques, mais la proximité topographique de leurs espèces associées (arrangées successivement) pourrait traduire la succession de grades évolutifs progressifs s'étageant dans le temps. En d'autres termes, les dichotomies successives qui montrent la proximité phylogénétique de différentes espèces d'un même genre illustreraient alors un clade et partant, donneraient une image cladistique des stades évolutifs d'une lignée. Cela pourrait être le cas pour Elfomys, car les branchements successifs suivent l'ordre chronologique. Il faut cependant analyser de plus près les caractères de ces espèces (cf. p. 52-53). Il serait nécessaire de faire de même pour les Pseudoltinomys éocènes : $P$. cosetanus est connu par des dents isolées représentant seulement $3 / 6$ des loci dentaires analysés ; P. mamertensis et $P$. phosphoricus sont représentés en nombre dans les gisements quercinois mais l'analyse de leur variabilité reste à faire.

- Au næud M (BP = 32), Pseudoltinomys phosphoricus est étroitement apparenté aux autres Pseudoltinomys (c'est-à-dire à $P$. cuvieri sur AE (Fig. 8) et au clade comprenant $P$. cuvieri avec les espèces oligocènes sur AG ; Fig. 7B) mais n'est pas inclus au sein du clade Pseudoltinomys. Le nœud est soutenu par 27 caractères NA et $7 \mathrm{~A}$. Parmi eux, on peut mettre en avant la connexion linguale du protolophe avec la postprotocrista chez $P$. phosphoricus, alors qu'elle se fait avec le protocône chez les autres Pseudoltinomys. Chez ces derniers, l'endolophe est présent tandis qu'il est absent chez $P$. phosphoricus. Si ce dernier montre une $\mathrm{m} 3$ plus courte que $\mathrm{m} 2$, ce n'est pas le cas chez les autres Pseudoltinomys, chez qui les $\mathrm{m} 3 \mathrm{~s}$ sont plus longues que les $\mathrm{m} 2 \mathrm{~s}$. Ces trois caractères sont communs avec les Theridomyinae, mais sont considérés par notre analyse comme des homoplasies. Pseudoltinomys phosphoricus se distingue encore par la présence constante de l'antérolophulide, limitant un SI et un ectolophide toujours présent et non interrompu ; le mésolophide y est court alors qu'il est long chez les autres espèces du genre.

- Le Nœud $N$ traduit la proximité phylogénétique du clade des autres Pseudoltinomys avec les Issiodoromys (Elfomys hartenbergeri est à présent attribué au genre Issiodoromys). Il est soutenu par 19 caractères NA et 8 A. Ces Pseudoltinomys montrent un degré d'hypsodontie supérieur à celui d'I. hartenbergeri, le paracône des DP4s est étiré buccolingualement alors qu'il est bulbeux chez I. hartenbergeri, le lophe central est plus haut, le postéroconule est présent sur les DP4s et les P4s des espèces de Pseudoltinomys, alors qu'il est absent chez I. hartenbergeri. Chez les autres espèces 
Email :

$P=$ Pauciserié

Pid = Pauciserié à décussation irrégulière

Pin = Pausisérié, prismes inclinés

$U=$ Unisérié

PsM =Pseudo-Multisérié

Longueur des foramens palatins antérieurs

Fpa1 = Très courts, éloignés du ramus antérieur

de l'arc zygomatique.

Fpa2 = Courts, jusque avant le bord antérieur du ramus.

Fpa3 = Moyens, jusqu'au niveau du bord antérieur de P4/DP4

Fpa4 = Longs, atteignant le milieu ou l'arrière de P4/DP4

Largeur du palais :

Lp1 > à 2 fois la largeur de $m 1$

Lp2 = à 2 fois la largeur de $\mathrm{m} 1$

Lp3 < à 2 fois la largeur de m1
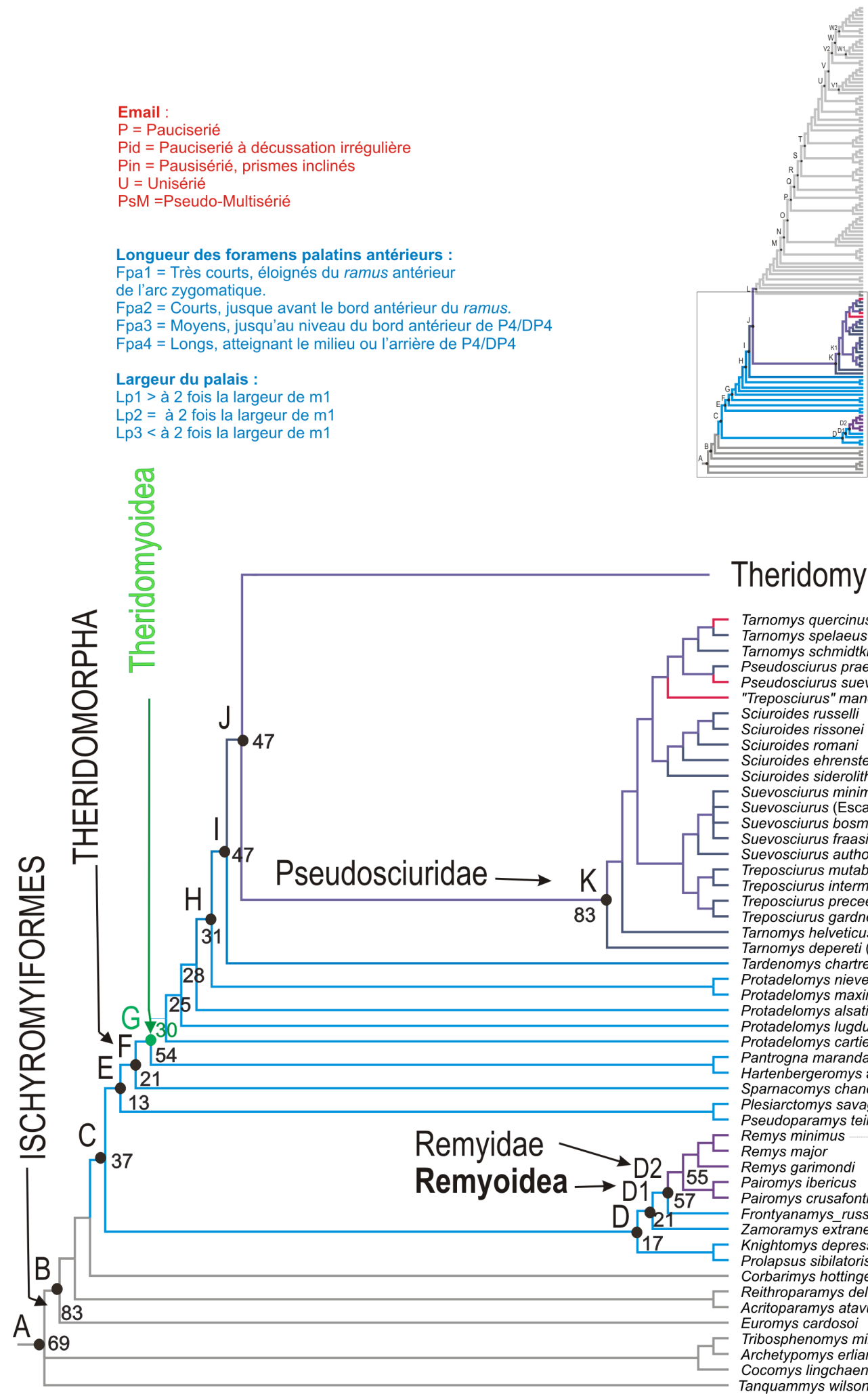

\section{Theridomyidae}

Tarnomys quercinus $\quad$ Fpa3 Tarnomys spelaeus P -Fpa3-4, Lp2 Tarnomys schmidtkittleri $\quad$ Fpa3, Lp2

Pseudosciurus praecedens Pseudosciurus suevicus - P "Treposciurus" manentis Sciuroides russelli Sciuroides rissonei Sciuroides romani Sciuroides ehrensteinensis Sciuroides siderolithicus Suevosciurus minimus Suevosciurus (Escamps) Suevosciurus bosmae Suevosciurus fraasi $\quad$ P -Fpa1, Lp1 Treposciurus mutabilis Treposciurus intermedius $\quad$ Fpa2, Lp2 Treposciurus preceei Treposciurus gardner

Tarnomys helveticus

Tarnomys depereti (Euzet) Tardenomys chartreuvensis Protadelomys nievesae Protadelomys nievesae Protadelomys alsaticus Protadelomys lugdunensis Protadelomys cartieri Pantrogna marandati

Hartenbergeromys aff. hautefeuillei Sparnacomys chandoni Plesiarctomys savagei Pseudoparamys teilhardi Remys minimus $P$ Remys major Remys garimondi Pairomys ibericus Pairomys ibericus Frontyan crusafonti Zrontyanamys_russell Knightomys extraneus Prolapsus sibilatoris $-\mathrm{P}$ Prolus sus Reithroparamys delicatissimus Reithroparamys delicatissimus
Euromys cardosoi Tribosphenomys minutus Archetypomys erlianensis Cocomys lingchaensis Tanquammys wilsoni

A

Figure 7. Analyse générale (AG) des 132 taxons considérés (groupes externes et groupe interne). Représentation de l'arbre de consensus strict obtenu à partir de 3 arbres équiparcimonieux, chacun de 2205 pas $(\mathrm{IC}=0,196 ; \mathrm{Ir}=0,762)$. Les couleurs des traits correspondent à la position géochronologique des taxons (gris $=$ Eocène inférieur, bleu $=$ Eocène moyen, violet $=$ Eocène supérieur, rouge $=$ Oligocène inférieur, brun = Oligocène supérieur) . Trois caractères non intégrés à l'analyse sont reportés sur l'arbre. Il s'agit de la microstructure de l'émail des incisives, de la longueur des foramens palatins antérieurs et de la largeur du palais (cf. légende sur la figure). Les principaux nœuds discutés sont reportés sur l'arbre, ainsi que leurs indices de bootstrap. Fig. 7A : de A à J ; Fig. 7B : de L à W. Les taxons de rang supra-spécifique sont reportés à partir du nœud qui marque leur émergence. 


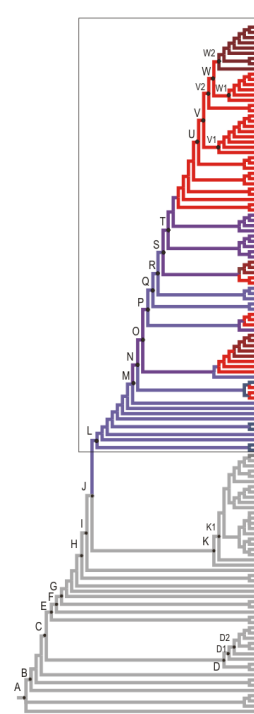

Tribu Archaeomyin

- Archaeomys arvernensis Archaeomys laurillardi

Archaeomys helveticus Archaeomys intermedius Archaeomys quercyi

PSM
PSM

— $\square 7$ Archaeomys / Blainvillimys huerzeler

PSM

Archaeomys / Blainvillimys sp. (MPF-LM)

4 W Archaechims/Bruci

$52 \quad-\square$ Protechimys lebratierensis Fpa4

V2

Protechimys gracilis

$\begin{array}{ll}\text { Protechimys variabilis } & \text { P à PsM Fpa4 } \\ \text { Protechimys blainvillei } & \end{array}$

$P$ à PsM

Toeniodus curvistriatus

Toeniodus curvistriatus

Blainvillimys stehlini

Blainvillimys campinsensis

Blainvillimys blainvillei

Blainvillimys avus

Blainvillimys helmeri (=

Blainvillimys gemellus

Blainvillimys heimersheimensis

Theridomys lembronicus

- Theridomys octogesensis

Theridomys major

- Paratheridomys margaritae

- Paratheridomys ludensis

- Bernardia marandati

- "Theridomys" (Trechomys) bonduelli

"Theridomys" (Trechomys) bonduelli Ham1-3

"Theridomys" (Isoptychus) aquatilis

- "Theridomys" golpe

"Theridomys" calafensis

Thalerimys headonensis

Thalerimys fordi

"Thalerimys" perrealensis

"Theridomys" brachydens

"Blainvillimy"s gousnatensis

"Blainvillimys "civracensis

"Blainvillimys" pseudosiderolithicus

- "Blainvillimys "rotundidens

- "Blainvillimys" langei

C Columbomys cuencae

Columbomys agustii

Columbomys lavocati

Sciuromys cayluxi

Sciuromys rigali

Patriotheridomys altus neboulensis

$P$ à PsM

- Patriotheridomys altus

Patriotheridomys sudre

Estellomys cansouni

Estellomys varleti

Ectropomys exigus

Ectropomys gliviformis

Oltinomys platyceps

Issiodoromys pseudanaema

Issiodoromys pseudanaema

Issiodoromys limognensis (Pech Desse)

1 Issiodoromys quercyi
Issiodoromys pauffiensis
Issodis

Isssodoromys puercyi

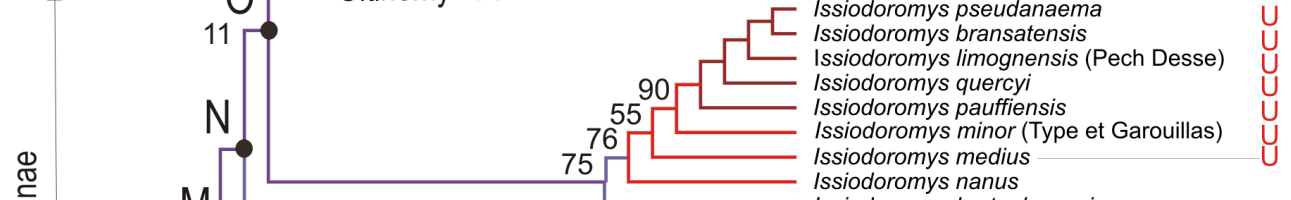

Issiodoromys nanus

Issiodoromys hartenbergeri

- Pseudoltinomys cuvieri

- Pseudoltinomys gaillard

Pseudoltinomys amblesi

Pseudoltinomys major

"Pseudoltinomys" phosphoricus__ Pid

(1)

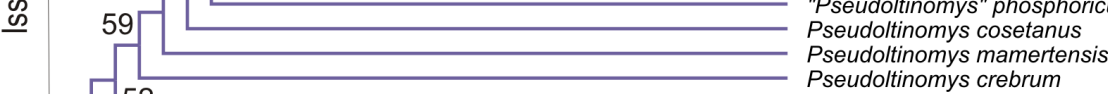
L $\nabla 55$
Elfomys tobieni
Elfomys catalaunicus
- Paradelomys santjaumensis

Elfomys parvulus

Sousfamille indet.

Fpa3, Lp2

Fpa4, Lp4

Fpa3, Lp3

U


d'Issiodoromys, le parastyle est présent, le protocône est étiré obliquement (alors qu'il est bulbeux chez Pseudoltinomys), le métalophule II est bucco-lingual (oblique vers l'arrière chez Pseudoltinomys) et le postérolophe est relié à l'extrémité linguale du métalophe ainsi qu'à la posthypocrista, tandis qu'il est connecté seulement à l'hypocône chez Pseudoltinomys. Les $\mathrm{p} 4 \mathrm{~s}$ sont de longueur à peine inférieure à celle des $\mathrm{m} 1 \mathrm{~s}$ de Pseudoltinomys, alors qu'elles sont nettement plus courtes chez Issiodoromys. Contrairement à $P$. phosphoricus, l'antérolophulide des molaires est absent chez les Pseudoltinomys et variablement présent chez Issiodoromys. Sur les dp4s, le mésostylide n'est connecté qu'à la crestide mésio-distale linguale chez Issiodoromys, alors qu'il est aussi connecté au mésolophide chez Pseudoltinomys phosphoricus.

- Le næud $O$ (20 caractères NA et 7 A) révèle la proximité phylogénétique des Issiodoromys oligocènes avec les Oltinomyinae. Si ce noeud est peu robuste $(\mathrm{BP}=11)$, par contre la topologie en clades successifs des espèces du clade Issiodoromys, d'I. hartenbergeri à I. pseudanaema I. bransatensis l'est beaucoup plus ( $\mathrm{BP}=75,76,55$ et 90 ). Cette organisation successive des branches correspond aux espèces se succédant dans le temps de la lignée principale des Issiodoromys, avec I. nanus comme lignée séparée du début de l'Oligocène (e.g., Vianey-Liaud, 1976). Les Oltinomyinae se séparent des Issiodoromys par la morphologie de la mandibule, traduisant un développement différent des masséters, avec l'incisive plus courte chez Issiodoromys que chez Oltinomys. L'endolophe n'a pas la même structure (deux éperons obliques chez Issiodoromys, un seul chez Oltinomys) et est nettement plus bas chez Oltinomys. Chez ce dernier, le postérolophe n'est lié qu'à l'hypocône, alors qu'il joint aussi l'extrémité linguale du métalophe chez Issiodoromys. Chez ce dernier, le SIII est moins profond que le SII, alors que les deux sont également peu profonds chez Oltinomys. Les m3s sont plus longues que les $\mathrm{m} 2 \mathrm{~s}$ chez Issiodoromys et d'égale longueur chez Oltinomys. La forme du protoconide est différente : pincée mésio-distalement chez Issiodoromys, étirée mésiodistalement chez Oltinomys. Le métalophide est fortement oblique, de buccomésial à disto-lingual chez Issiodoromys et transverse chez Oltinomys. Le mésolophide des dp4s est connecté au mésostylide chez Oltinomys alors qu'il est libre chez Issiodoromys.

- Nocud $P(\mathrm{BP}=20 ; 35$ caractères NA, 4 A $)$. Il traduit la proximité des Oltinomyinae avec le genre Estellomys, représenté par deux espèces apparentées $(\mathrm{BP}=99), E$. cansouni et $E$. varleti, cette dernière antérieurement attribuée à Theridomys (Hartenberger \& Louis, 1976) puis Blainvillimys (Comte et al., 2012). Le protocône montre un sommet pointu chez Oltinomys, alors qu'il est étiré obliquement à partir de l'antérolophe chez Estellomys. Le métaconule est bulbeux chez Oltinomys et étiré bucco-lingualement chez Estellomys. La mure, absente chez Oltinomys, est ébauchée chez Estellomys, alors que l'endolophe, présent et mésio-distal chez Oltinomys, est variablement présent et réduit à un éperon issu du flanc distal du protocône chez Estellomys. Le paracône et le métacône sont étirés bucco-lingualement chez ce dernier, alors qu'ils sont faiblement étirés mésio-distalement chez Oltinomys. Les $\mathrm{p} 4 \mathrm{~s}$ sont plus courtes que les $\mathrm{m} 1 \mathrm{~s}$ chez Oltinomys, égales ou plus longues chez Estellomys. Sur les dp4s, le métaconide est en position mésio-linguale, le protoconide est étiré mésiodistalement chez Oltinomys, tandis que le métaconide est lingual et le protoconide est étiré obliquement chez Estellomys.
Estellomys est caractérisé aussi par la présence de crestides le long de l'axe des dents dans le sII. D'autres caractères séparent ces deux genres : connexions ou séparations de l'ectolophide, du mésostylide ou du sinuside avec le sIV.

Estellomys apparaît étroitement apparenté au genre Patriotheridomys au nœud $Q$ de $\mathrm{AG}(\mathrm{BP}=34 ; 31$ caractères NA et $7 \mathrm{~A}$ ). Cette relation se retrouve sur l'analyse réduite aux taxons éocènes (AE), mais dans ce cas Bernardia (genre du Priabonien basal) se positionne entre les deux. Or sur AG, Bernardia se place au sein des Theridomys oligocènes, alors qu'il s'en distingue clairement par la présence de l'endolophe ; mais les branchements des différents genres de Theridomys sont très peu robustes (BP de 3 à 5). Par ailleurs, sur AE, le clade Patriotheridomys est étroitement apparenté aux premiers Blainvillimys : sur AG, les Columbomyinae - tous oligocènes viennent s'intercaler entre les Patriotheridomys et les premiers Blainvillimys. Estellomys diffère des Patriotheridomys par un moindre degré d'hypsodontie, par un antérolophe et un postérolophe plus bas, la présence del'endolophe bien développé et la mure seulement ébauchée. Patriotheridomys montre une P4 plus grande que M1, le paraconule est étiré bucco-lingualement dans le protolophe, alors qu'il est bulbeux chez Estellomys. Le métaconule est absent et le SIV réduit, le mésocône et le postéroconule sont présents chez Patriotheridomys. Le postérolophe n'est lié qu'à l'hypocône chez Estellomys, alors qu'il joint aussi l'extrémité linguale du métalophe chez Patriotheridomys. Les $\mathrm{p} 4 \mathrm{~s}$ montrent un fort antéroconide ainsi que l'attache mésiale du métalophide chez ce dernier, alors que l'antéroconide est réduit et l'attache linguale du métalophide se fait sur le sommet du protoconide chez Estellomys. Le mésolophide, court, montre des interruptions chez ce dernier, alors qu'il est long et continu chez Patriotheridomys. Les relations entre les espèces de Patriotheridomys sont robustes $(\mathrm{BP}=99)$. Comme pour les Issiodoromys, elles sont organisées en succession chronologique. La plus ancienne ( $P$. sudrei, la moins hypsodonte, avec les DP4s et dp4s les plus courtes et les plus simples) apparaît comme l'espèce la plus basale du clade Patriotheridomys, les deux autres espèces étant représentées comme un clade terminal, bien que $P$. neboulensis soit plus ancienne que $P$. altus. Cette dernière est l'espèce la plus hypsodonte, avec des DP4s et dp4s très longues et complexes.

- Noud $R(\mathrm{BP}=51 ; 37$ caractères NA et $6 \mathrm{~A})$ traduit la proximité phylogénétique des Patriotheridomys avec les Columbomyinae. Ils diffèrent par la structure mandibulaire, les différences d'épaisseur de l'émail sur les flancs opposés des lophes et lophides chez Patriotheridomys mais pas chez les Columbomyinae. Chez Patriotheridomys, les molaires supérieures montrent la connexion du protolophe avec le sommet du protocône, alors que cette connexion se fait avec l'extrémité de la postprotocrista chez les Columbomyinae. Cette dernière configuration est ensuite la règle pour tous les Theridomyinae, tout comme l'absence d'endolophe, alors que ce dernier peut exister, vestigial, chez Patriotheridomys. Le sinus est profond chez Patriotheridomys et plus court chez les Columbomyinae. Sur les DP4s de Patriotheridomys, on peut noter une crestule mésiale au métacône, laquelle est absente chez les Columbomyinae. Chez ces derniers, le métalophule II est transverse, alors qu'il est fortement oblique distalement chez Patriotheridomys. Le postérolophe est connecté au métacône et au métalophe et séparé de l'hypocône chez Patriotheridomys, alors qu'il joint l'extrémité linguale du métalophe et l'hypocône, sans être connecté directement au 
métacône chez les Columbomyinae. Les incisives sont plus longues chez les Columbomyinae, l'antésinuside est absent des $\mathrm{dp} 4 \mathrm{~s}$ et présent sur les molaires de ces derniers, la crestide préprotoconide et le métalophulide I sont absents des $\mathrm{dp} 4 \mathrm{~s}$ de Patriotheridomys, mais présents chez les Columbomyinae, alors que la cuspide surnuméraire mésiale au postérolophide est présente seulement chez Patriotheridomys.

- Noud $S(\mathrm{BP}=34 ; 46$ caractères NA et 7 A) traduit la proximité phylogénétique des Columbomyinae avec un premier groupe basal de Theridomyinae, celui de «Blainvillimys ». Ils diffèrent par la morphologie de la mandibule qui présente une branche montante plus basse chez les Columbomyinae et une longueur de l'incisive moindre que chez "Blainvillimys » où elle naît en arrière de $\mathrm{m} 3$. L'hypsodontie est moins forte chez ces espèces de « Blainvillimys » que chez les Columbomyinae. Sur les dents vierges, le protocône est pointu et faiblement comprimé mésio-distalement chez le columbomyiné Sciuromys, alors qu'il est étiré obliquement dans le prolongement de l'antérolophe chez les «Blainvillimys ». Le paracône et le métacône sont bulbeux chez Sciuromys et étirés bucco-lingualement chez les « Blainvillimys ». L'hypocône des molaires et des DP4s des Columbomyinae est crescentiforme, son flanc lingual étant pincé mésio-distalement ; les dents déciduales (DP4s et dp4s) sont nettement plus courtes que les $\mathrm{m} 1 \mathrm{~s}$ chez les Columbomyinae et nettement plus longues chez «Blainvillimys ». L'antéroconide et le cingulide mésiobuccal sont absents des dp4s mais présents et forts sur les molaires de Columbomyinae ; l'antéroconide est présent sur les dp4s et réduit sur les molaires chez «Blainvillimys ». Le mésolophide « vrai » est absent chez les Columbomyinae (présence variable d'un court néo-mésolophide); il est présent et long chez « Blainvillimys ». Le métalophide est très oblique linguomésial à disto-buccal chez les Columbomyinae, alors qu'il est transverse chez ces «Blainvillimys ». L'entolophide des dp4s-p4s est connecté au mésoconide ou à l'ectolophide (au mésoconide pour les molaires) chez les Columbomyinae, alors qu'il se connecte à l'extrémité de la préhypocristide chez les « Blainvillimys ».

- Le Noud T $(\mathrm{BP}=22 ; 16$ caractères NA et $6 \mathrm{~A})$ exprime la proximité phylogénétique du précédent clade des «Blainvillimys » avec un clade regroupant les espèces de Thalerimys plus "Theridomys " brachydens, ce dernier apparaissant comme espèce sœur des Thalerimys. Ces "Theridomys " - Thalerimys sont moins hypsodontes et l'épaisseur de l'émail est régulière, alors que les «Blainvillimys » sont plus hypsodontes et montrent une différence d'épaisseur de l'émail sur les flancs opposés des lophes et lophides ainsi que des sinus et sinuside. Les DP4s des « Blainvillimys » montrent un antésinus, alors que les Thalerimys + « Theridomys" brachydens n'en ont pas. Le mésostyle est réduit sur tous les loci chez «Blainvillimys » alors qu'il est fort chez Thalerimys + «Theridomys » brachydens. La longueur des m3s est plus réduite chez «Blainvillimys ». Le sI des dp4s est fermé seulement lingualement chez Theridomys-Thalerimys; il l'est aussi mésialement et buccalement chez "Blainvillimys". Le paraconide et le métaconide sont encore saillants bien qu'étirés dans la continuité des métalophide et entolophide chez «Theridomys » - Thalerimys, alors qu'ils n'émergent pas au-dessus de ces lophides chez Blainvillimys. La topologie de ces « Theridomys »+ Thalerimys est un peu différente sur les arbres de l'analyse partielle AE. Selon les résultats de cette analyse (Fig. 8), « Thalerimys » perrealensis apparaît comme l'espèce sœur du clade regroupant un sous-clade de Thalerimys (Th. fordi et Th. headonensis) et un sous-clade de Theridomys («T. » bonduelli, « T. » brachydens - «T. » golpei). Sur AO (Fig. 9), comme Thalerimys est exclu, les branchements des Theridomys sont différents, notamment celui de «T. » golpei, qui émerge loin après « T. » brachydens.

Sur AG, les nœuds suivants le nœud $\mathrm{T}$ sont très peu robustes (BP de 5 à 3). La topologie montre un arrangement en clades successifs des autres Theridomys. Le premier de ces nœuds distingue un clade «T. » calafensis - «T. » golpei. Aux nœuds suivants émergent d'abord «T. . aquatilis puis « T. » bonduelli. S'ensuit un sous-clade incluant Bernardia : ce dernier apparaît comme le taxon frère de «T. . margaritae + «T. » ludensis. Le dernier sous-clade qui suit regroupe Theridomys major, $T$. lembronicus et $T$. octogesensis, un regroupement dont la hiérarchisation pourraît illustrer la lignée des chrono-espèces Theridomys major-lembronicus-octogesensis, qui comprend l'espèce-type du genre. Si l'émergence de la branche à partir des Theridomys précédents sur l'arbre est peu robuste (BP $=5)$, les relations entre ces trois espèces le sont en revanche beaucoup plus $(\mathrm{BP}=96)$.

- Le Noud U (BP $=39 ; 24$ caractères NA et $2 \mathrm{~A})$ illustre les liens de parenté étroits entres les derniers Theridomys et les Blainvillimys oligocènes (sauf langei) qui forment un clade, mais également avec les Protechimys et les Archaeomys (cette association sera désignée " BPA » par la suite). On retrouve la distinction Theridomys/Blainvillimys remarquée au nœud T. Les Theridomys sont moins hypsodontes et l'épaisseur de l'émail est régulière, alors que les Blainvillimys sont plus hypsodontes, avec une différence d'épaisseur de l'émail sur les flancs opposés des lophes et des lophides, ainsi que sur ceux du sinus et du sinuside. Le sinus est plus court chez Theridomys que chez BPA. Le mésostyle est absent sur les DP4s de Theridomys et présent chez BPA. Le S0 existe chez les DP4s des taxons du clade BPA et il est subdivisé en deux ou trois parties chez Theridomys. Chez ces derniers, le métacône est étiré obliquement (de buccomésial à disto-lingual) alors qu'il est fusionné dans le métalophe sur les DP4s et molaires supérieures des taxons du clade BPA. Le mésolophule des Theridomys est connecté à un renflement de l'extrémité de la préhypocrista, alors que ce renflement est absent chez les BPA. Le postérolophe est relié seulement à l'hypocône chez Theridomys, alors qu'il l'est aussi avec le métalophe chez les taxons du clade BPA. L'antéroconide est absent des $\mathrm{p} 4 \mathrm{~s}$ des Theridomys et présent chez les BPA. Le sI des dp4s est fermé lingualement, mésialement et buccalement chez Theridomys et il est ouvert lingualement en avant du métaconide chez les BPA. Il est précédé de synclinide(s) en avant de sI chez Theridomys mais pas chez les taxons du clade BPA. Le sI est toujours présent et profond chez Theridomys ; il l'est variablement et très peu profond, souvent réduit à un îlot, chez les taxons du clade BPA. Sur les dp4s, sI et sII sont séparés chez Theridomys et communiquent chez les BPA. Le sIV est séparé du sinuside par l'ectolophide chez Theridomys, alors qu'une communication, même peu profonde, existe chez les taxons du clade BPA.

- Le Noud $V(\mathrm{BP}=28 ; 22$ caractères NA et $3 \mathrm{~A})$ constitue le clade BPA et il traduit avant tout la proximité phylogénétique du clade des Blainvillimys (comprenant le type du genre) avec le clade des Archaeomyini, lequel inclut Protechimys + Archaeomys/Blainvillimys + Archaeomys. Les taxons 


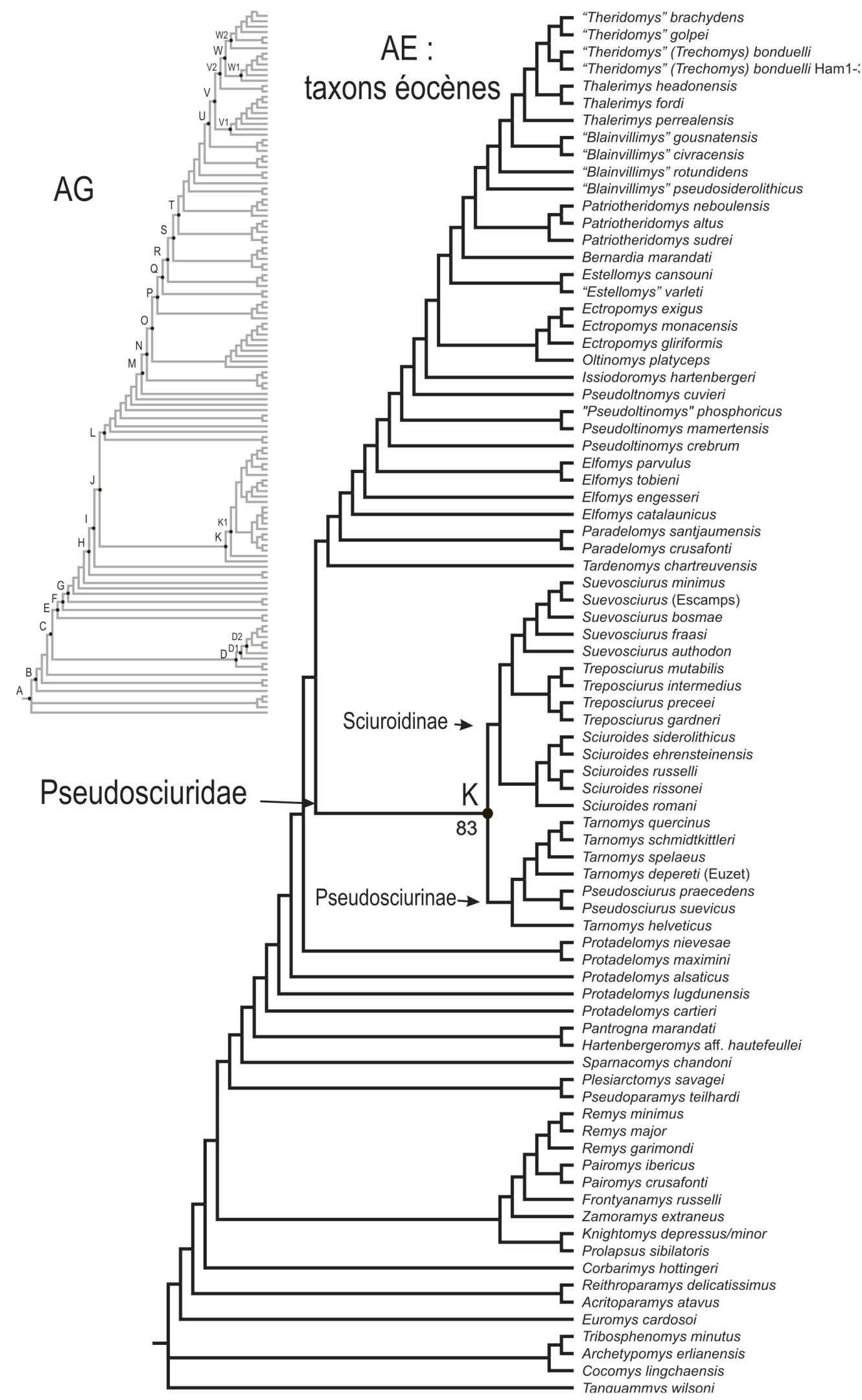

Figure 8. Analyse partielle des taxons éocènes (+ groupes externe et de branchement) $=\mathrm{AE}$. Représentation de l'arbre de consensus strict obtenu à partir de 8 arbres équiparcimonieux, chacun de 1725 pas (IC $=0,225 ; \mathrm{Ir}=0,666)$. AG est donnée en réduction à titre de comparaison. 
de ces deux clades se distinguent par la structure de leur mandibule (crêtes massétériques également développées versus crête massétérique inférieure plus forte), traduisant un fonctionnement différent des muscles masséters. Sur les DP4s, l'antérostyle est absent chez Blainvillimys, présent chez les Archaeomyini. Le mésostyle est fort chez ces derniers, plus petit chez les Blainvillimys. La mure est haute et complète chez Blainvillimys, interrompue chez les autres ; le mésocône est présent chez Blainvillimys seulement. Le sinus occupe totalement la largeur de la dent chez les Archaeomyini, alors qu'il est moins étendu chez les Blainvillimys. Les SII et SIII sont également profonds chez Blainvillimys, le SIII l'est moins que le SII chez les Archaeomyini. Le postérolophe est connecté à l'extrémité linguale du métalophe et à l'hypocône et séparé du métacône chez Blainvillimys; il relie le métacône à l'hypocône chez les Archaeomyini. Les synclinides sI et sII sont connectés sur les dp4s peu usées des Archaeomyini et séparés chez Blainvillimys. Le métalophide des dp4s est complet chez ce dernier et incomplet chez les autres. Sur dp4s et $\mathrm{p} 4 \mathrm{~s}$, le mésostylide est connecté uniquement au mésolophide chez Blainvillimys, alors qu'il est aussi connecté à la crestide pré-métaconide mésio-distale chez les Archaeomyini.

.) Le Noud V1 (BP = 26) correspond au clade des espèces de Blainvillimys de l'Oligocène. La branche la plus basale de ce clade des Blainvillimys oligocènes est $B$. heimersheimensis (MP24). Les autres espèces regroupées dans ce genre s'organisent en branches successives ( $B$. gemellus, B. gregarius, B. varians-helmeri ; MP22-MP23) et les formes les plus terminales correspondent aux espèces les plus récentes (Rupélien terminal - MP24 et Chattien basal MP25 et MP26). Il s'agit de Blainvillimys avus (MP24) qui apparaît comme l'espèce sœur des trois espèces du clade terminal. Ce dernier est constitué de B. stehlini (MP26) et du couple Blainvillimys blainvillei - B. campinsensis (les deux rapprochées de MP25). Cette dernière apparaît ici comme une espèce du genre Blainvillimys et non de Theridomys comme proposé initialement par Arbiol (1993). Cette représentation ne correspond pas totalement à celle des trois lignées reconnues jusqu'ici (e.g., Bahlo, 1975 ; Vianey-Liaud, 1972a et b, 1989, 1998), ce qui sera discuté ultérieurement. Blainvillimys heimersheimensis se distingue donc des autres Blainvillimys au nœud V1, par 14 caractères NA et deux A. Aux DP4s, il s'agit de la présence d'un antérostyle réduit (il est absent chez les autres) et de l'absence d'antésinus (il est présent chez les autres Blainvillimys oligocènes). Au niveau des prémolaires et molaires supérieures, les différences s'expriment essentiellement sur le développement et les connexions des crêtes linguales (postparacrista et prémétacrista) et du mésostyle, ainsi que sur l'enfoncement bucco-lingual du sinus, plus faible chez $B$. heimersheimensis. Les différences sont plus marquées pour les dp4s inférieures : l'antésinuside est plus profond chez ce dernier, avec les sI et sII connectés seulement sur les dp4s peu usées chez le premier, alors qu'il l'est toujours chez les autres Blainvillimys; le s0 de ces dp4s est toujours présent chez $B$. heimersheimensis, absent chez les autres Blainvillimys oligocènes.

.) Le noud V2 $(\mathrm{BP}=41 ; 30$ caractères NA et $4 \mathrm{~A})$ représente l'autre partie du clade des Archaeomyini, les Protechimys + Archaeomys et Archaeomys/Blainvillimys, avec, comme rameau basal, les deux espèces de Toniodus. Ce dernier se distingue des autres Archaeomyini par la présence d'un palais rectangulaire (comme celui de tous les autres théridomorphes), alors que chez les Archaeomyini il est trapézoïdal, plus étroit à l'avant qu'à l'arrière. Les dents de
Tœniodus sont moins hypsodontes que celles des Archaeomyini et le sinus est moins long bucco-lingualement. Sur les DP4s de Toniodus, l'antésinus est absent alors qu'il est présent chez les Archaeomyini. Le parastyle, absent sur les DP4s, est présent sur les M1-2s de Toniodus et c'est l'inverse pour les Archaeomyini. La mure est absente sur les DP4s de Toniodus, mais présente chez les Archaeomyini. SI est relativement profond sur les $\mathrm{P} 4 \mathrm{~s}$ de Tœniodus et réduit chez les Archaeomyini. Le SIV est oblique chez Toniodus, réduit à un îlot ovale à circulaire chez les Archaeomyini. L'hypocône est étiré en crête oblique, anguleuse chez Toniodus et régulièrement courbée chez les Archaeomyini. Les incisives inférieures sont plus courtes chez les Archaeomyini (origine sous m3). Le sI des dp4s est complètement clos chez Tœniodus, alors qu'il est ouvert lingualement, en avant du métaconide chez les Archaeomyini. Le sI est presque toujours présent sur les m1-2s de Toeniodus et absent à variablement présent chez les Archaeomyini (sur moins de $50 \%$ des $\mathrm{m} 1-2 \mathrm{~s}$ ). Le s0 est présent sur les dp $4 \mathrm{~s}$ des Archaeomyini et absent chez Toniodus. Le mésoconide est absent tandis qu'un néomésoconide est présent sur les dents inférieures de Toniodus, les deux sont absents sur celles des Archaeomyini. Le mésostylide est connecté au mésolophide et à la postmétacristide chez Toniodus et seulement au mésostylide chez les Archaeomyini. Une encoche profonde fait communiquer le sIV avec le sinuside chez Tœniodus, alors qu'ils sont séparés par l'ectolophide chez les Archaeomyini. L'entolophide est connecté à l'extrémité de la préhypocristide chez les Archaeomyini et au mésoconide ou à l'ectolophide chez Toniodus.

- Le Noud $W(\mathrm{BP}=52)$ sépare les clades Protechimys et Archaeomys. Chacun des genres montre une topologie en sous-clades de leurs espèces respectives. Ces deux genres se distinguent par 17 caractères NA et deux A. Il faut noter plusieurs homoplasies, témoignant d'acquisitions parallèles de caractères, tels que :

- l'acquisition de cément chez Protechimys major et Archaeomys,

- le parastyle, disparu au nœud V et présent chez la première branche d'Archaeomys/Blainvillimys seulement,

- le SII vestigial au nœud W, égal à SIII chez les Archaeomys terminaux, mais aussi chez les Protechimys chronologiquement les plus récents,

- l'enfoncement maximum du sinus jusqu'au bord buccal chez les Archaeomys terminaux et chez les Protechimys chronologiquement les plus récents,

- la très grande longueur des DP4s chez tous les Protechimys, sauf P. truci et chez les Archaeomys du Chattien supérieur,

- la réduction de la taille de l'antéroconide des p4s chez tous les Protechimys et les Archaeomys terminaux, mais aussi chez B. avus, $T$. golpei, $O$. platyceps et $E$. cansouni au sein des Theridomyidae,

- la préparacrista et la postmétacrista des dents supérieures présentes chez les Protechimys mais aussi chez les Blainvillimys, Theridomys et Issiodoromys terminaux, la plupart des Pseudosciuridae (en ce qui concerne la préparacrista) et Paradelomys crusafonti,

- la prémétacrista est absente chez les Archaeomys et aussi chez les Issiodoromyinae basaux, à plusieurs reprises, ou au sein des Ischyromyoidea et Remyidae. 


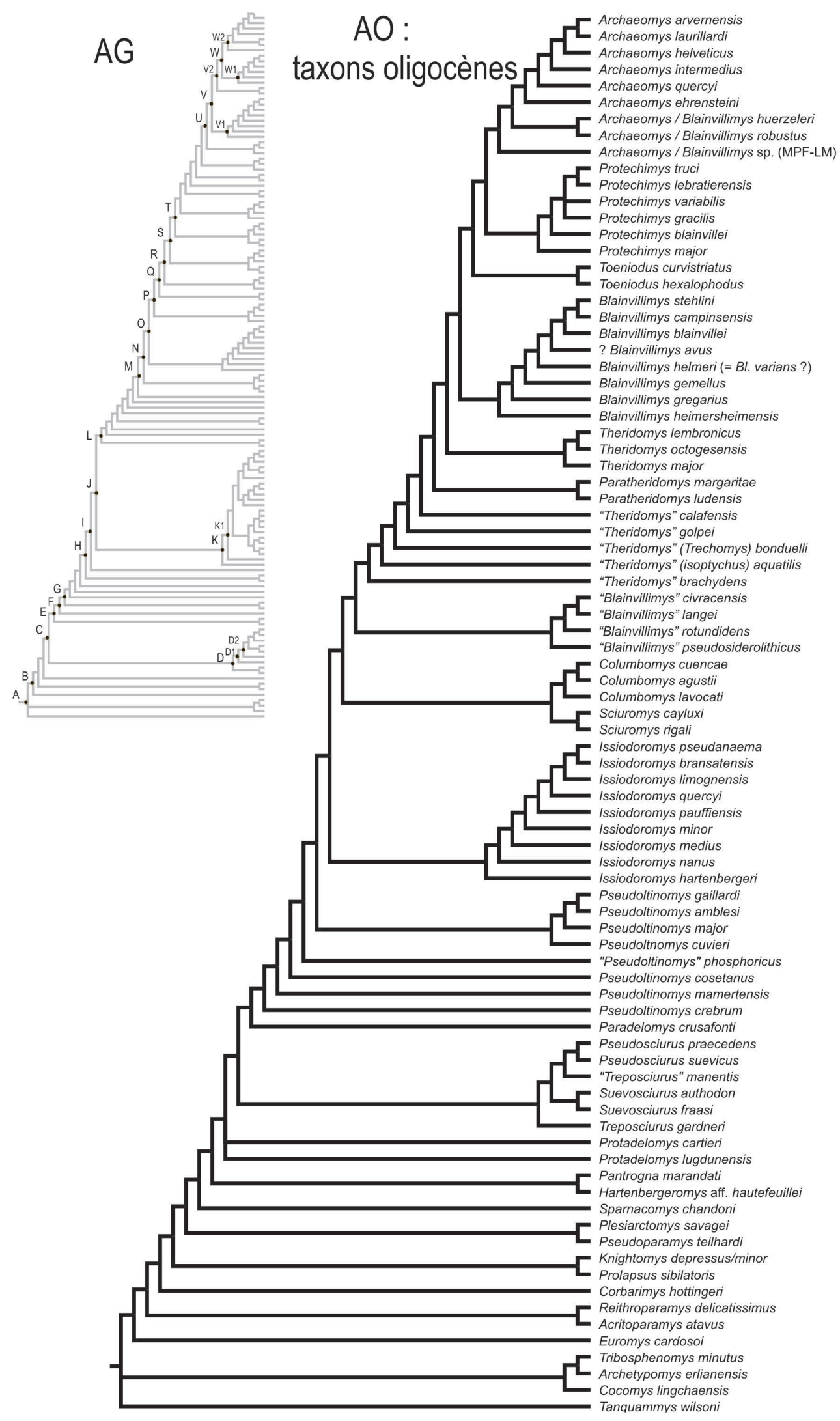

Figure 9. Analyse partielle des taxons oligocènes (+ groupes externe et de branchement) $=$ AO. Représentation de l'arbre de consensus strict obtenu à partir de 8 arbres équiparcimonieux, chacun de 1359 pas $(\mathrm{IC}=0,296 ; \mathrm{Ir}=0,752)$. AG est donnée en réduction à titre de comparaison. MPF-LM: Mas-de-Pauffié et Les-Milles. 
En dehors de ces caractères homoplasiques, l'extension du cément est différente chez Protechimys et Archaeomys. Le parastyle redevient fort seulement chez les Archaeomys au sein des Archaeomyini, mais il l'est aussi dans différentes branches au sein des Theridomorpha et chez quelques Ischyromyoidea. Le SI des DP4s est toujours présent chez les Protechimys, même les plus hypsodontes. Le sinus des molaires supérieures apparaît plus long bucco-distalement que chez Archaeomys. Or c'est seulement le cas chez $P$. blainvillei et $P$. major, les plus récents des Protechimys, mais pas chez les formes anciennes $P$. truci, $P$. lebratierensis ou $P$. variabilis. La topologie des Protechimys les classe ici dans l'ordre inverse de la succession chronologique des espèces, au contraire des tendances évolutives proposées (i.e., allongement du sinus). Protechimys major est contemporain du plus ancien des Archaeomys/ Blainvillimys qui montre effectivement un sinus moins long. Les autres espèces d'Archaeomys, de la plus ancienne à la plus récente, montrent un sinus plus long, qui dépasse en longueur celui de P. major. Enfin, si Protechimys montre encore un ectolophide, ce n'est plus le cas chez Archaeomys.

.) Au Noud W1 (BP = 46), le clade correspond aux espèces du genre Protechimys : leur topologie est organisée en branches successives, en ordre inverse du registre stratigraphique (cf. ci-dessus). Cette topologie est soutenue par 11 caractères NA et $3 \mathrm{~A}$. Parmi eux, la première branche, $P$. major, se distingue des autres par des dents à couronne plus haute, la présence de cément, l'absence de prémétacrista, le SI des $\mathrm{P} 4 \mathrm{~s}$ variablement présent, alors qu'il l'est toujours chez les autres, le sI des dp $4 \mathrm{~s}$ occupant toute la largeur du lobe antérieur, la disparition du sI des m1-2s et du s0 des dp4s.

.) Au Noud W2 (BP=67), le clade correspond aux espèces du genre Archaeomys. Comme précédemment, les espèces sont également organisées en branches successives. L'attribution générique des trois premières a varié entre les genres Blainvillimys et Archaeomys. Elles portent ici le nom double «Archaeomys/Blainvillimys » (cf. Vianey-Liaud, 2015), en attendant une révision de ce groupe des Archaeomyini (Mödden, 1993). La première branche est une espèce non nommée d'Archaeomys/Blainvillimys, contemporaine de Protechimys major. Elle se distingue de la branche suivante (les deux autres Archaeomys/Blainvillimys formant un clade : A./B. huerzeleri $+A . / B$. robustus) seulement par neuf caractères NA et un A. Il s'agit d'abord de la moindre extension du cément sur la couronne et du plus faible degré d'hypsodontie. Le SV est absent sur les DP4s, alors qu'il est présent chez les deux autres espèces d'Archaeomys/Blainvillimys; le SIV des M1-2s est réduit à un îlot et plus allongé chez les deux autres espèces d'Archaeomys/Blainvillimys, le SI des P4s est toujours présent, alors qu'il l'est variablement chez les deux autres espèces d'Archaeomys/Blainvillimys. Aux dp4s inférieures, sI et sII sont séparés, alors qu'ils sont connectés chez les deux autres espèces d'Archaeomys/Blainvillimys.

Les espèces suivantes, toutes en ordre de branchement successif, sont attribuées au genre Archaeomys. La première (Archaeomys ehrensteini) n'a pas le SV aux DP4s, comme Archaeomys/Blainvillimys sp. Ce SV est ensuite présent sur les $\mathrm{DP} 4 \mathrm{~s}$ des cinq autres espèces d'Archaeomys. Il est absent des $\mathrm{P} 4 \mathrm{~s}$ jusqu'à $A$. quercyi et présent ensuite (de $A$. intermedius à $A$. arvernensis). Le SI de $\mathrm{P} 4$ est toujours présent chez $A$. ehrensteini, variablement chez $A$. quercyi et absent pour les espèces qui suivent. La mure est présente sur toutes les $\mathrm{P} 4 \mathrm{~s}$ et se situe très près du bord buccal, avec le sinus occupant la largeur totale de la dent chez les deux espèces terminales
(A. laurillardi et A. arvernensis). Aux M1-2s, SII et SIII sont distincts chez Archaeomys ehrensteini, SII devenant vestigial ou absent chez les autres, entraînant ainsi une extension $\mathrm{du}$ sinus qui occupe toute la largeur de la surface occlusale. Le SIII s'ouvre buccalement à partir d'A. quercyi et le SIV seulement chez les deux espèces terminales. Le SV est absent des molaires chez $A$. ehrensteini et la plupart de celles d'A. quercyi, variablement présent chez les autres. A partir d'A. intermedius, les incisives inférieures sont très courtes (débutent mésialement à $\mathrm{m} 3$ ), l'antéroconide des $\mathrm{p} 4 \mathrm{~s}$ est petit et le sI des m1-2s est absent. Sur les dp4s d'A. ehrensteini, le sI des dp4s est clos de tous côtés et subdivisé par des crêtes accessoires, alors qu'il est ouvert lingualement, en avant du métaconide, chez tous les autres Archaeomys, comme chez la plupart des Protechimys (sauf $P$. major et lebratierensis, chez qui il est ouvert lingualement et buccalement). C'est aussi le cas chez divers autres Theridomyoidea tels que Theridomys lembronicus, Blainvillimys gemellus et Pseudosciurus precedens.

\section{EMERGENCE DES THERIDOMYOIDEA}

A partir du milieu du $20^{\text {ème }}$ siècle, les Theridomyoidea ont été considérés comme issus de représentants européens de la superfamille des Ischyromyoidea (e.g., Lavocat, 1951, 1955 ; Wood, 1962). Au cours de l'Eocène inférieur, l'aire de répartition de cette super-famille englobait principalement l'Amérique $\mathrm{du}$ Nord et l'Europe, quelques formes étant présentes en Asie. Lavocat discutait aussi de leur relation de parenté possible avec les anomaluridés africains, hystricomorphes, sciurognathes et pentalophodontes, comme le sont aussi de nombreux Theridomyidae. S'il n'écartait pas la possibilité d'une parenté proche entre anomalures et théridomorphes, il l'envisageait seulement « provisoirement », faute d'arguments qui permettraient de trancher entre parenté et convergence.

Hartenberger (1990) proposait une relation de parenté des Anomaluroidea avec les Theridomyoidea. Dans cet article, il utilise indifféremment le niveau superfamilial (Theridomyoidea) ou familial (Theridomyidae) pour désigner les taxons regroupés dans les familles actuelles Theridomyidae et Pseudosciuridae. Par ailleurs, pour lui, ces deux familles n'offraient pas un cadre systématique pertinent pour tous les taxons de «théridomorphes » sensu Lavocat, dont il avait proposé antérieurement une classification subfamiliale (Hartenberger, 1971a ; 1973b). En 1990, il se base sur une « analyse de parcimonie » des états (plésiomorphe-intermédiaire-dérivé) de quatre caractères crâniens +13 caractères dentaires, qu'il attribue aux six super-familles de rongeurs présentes à l'Eocène moyen et supérieur. Les dits caractères ne sont pas clairement définis, pas plus que ne le sont les taxons sur lesquels ils sont observés au sein des super-familles. Dans la même analyse, les " Phiomyoidea " (sensu Hartenberger, $1990=$ phiomorphes africains) sont éloignés des Ctenodactyloidea et sortent comme groupe frère des anomaluroïdes + theridomyoïdes. Enfin, Hartenberger remarque le « caractère brutal » de l'apparition des " théridomyoïdés », car diversifiés dès l'origine, avec trois genres pénécontemporains (MP13-14) : Protadelomys, Treposciurus et Paradelomys. Rapprochant ce dernier fait de la découverte dans l'Eocène moyen de Santa Clara en Espagne d'un nouveau rongeur, interprété à l'époque comme un possible représentant européen des Chappatimyidae asiatiques (Peláez-Campomanes et al., 1989), Hartenberger envisage « un mouvement migratoire de grande amplitude » des rongeurs depuis l'Asie. 
Dans un article sur les genres de rongeurs regroupés auparavant dans les Oltinomyinae par Bosma \& SchmidtKittler (1972), le partage des caractères du trigonide des dents inférieures de ce nouveau rongeur d'Espagne avec les Theridomyidae (sensu Hartenberger, 1971) est souligné, alors que l'hypoconulide fort et les caractères des molaires supérieures semblent primitifs (Vianey-Liaud et al., 1994). Ces derniers auteurs ont envisagé une aire de différenciation des Theridomyidae (sensu Hartenberger, 1971) dans la péninsule ibérique. Ce rongeur d’Espagne (PeláezCampomanes et al., 1989) a finalement été décrit de façon formelle par ses découvreurs, sous le nom de Zamoramys extraneus (Peláez-Campomanes \& López-Martinez, 1996). Comme Hartenberger (1990), ces auteurs ont rapproché Zamoramys des Chappattimyidae, notamment Birbalomys, sur la base d'une évaluation cladistique des quelques caractères dentaires recensés. Cette dernière étude fut plus précise que celle d'Hartenberger quant à la définition des caractères, à la dénomination des genres considérés et aux caractéristiques de l'arbre obtenu, mais elle est restée cependant très limitée par le faible nombre d'espèces (9) et de caractères (9), même si quelques autres caractères ont été discutés a posteriori (non inclus dans leur analyse). Par ailleurs, comme avec Hartenberger (1990), la polarité des caractères fut définie a priori. L'analyse de certains des caractères prête à discussion, notamment ceux des molaires inférieures (liaisons du mésoconide ; liaisons de l'hypolophide ; absence de définition de l'ectolophide). L'arbre obtenu montre une dichotomie Ischyromyidae + Gliridae et cténodactyloïdes + Theridomyidae, Zamoramys étant le genre frère des deux Theridomyidae considérés.

Dans une analyse cladistique de la radiation Eocène des rongeurs, plus exhaustive (91 taxa, 108 caractères), Zamoramys se placera du côté des Ischyromyiformes dans la dichotomie basale des rongeurs et pas du tout avec les chappattimyidés liés aux Ctenohystrica (Marivaux et al., 2004 : fig. 2). Dans la présente analyse, les genres Zamoramys, Frontyanamys, Remys et Pairomys constituent un clade (Remyoidea) au sein des Ischyromyoidea, avec comme groupe frère les Sciuravidae. Ces genres basaux européens émergent bien avant la radiation initiale des Theridomorpha ou des Theridomyoidea (Figs. 7A et 8).

Enfin, depuis 1996, des découvertes documentent des Theridomyoidea dans plusieurs localités du Lutétien : Protadelomys nievesae dans le site de Casa-Rámon (MP11/12; (Peláez-Campomanes, 1996), plus ancien donc que Zamoramys et $P$. maximini dans celui de Saint-Maximin (Escarguel, 1998 ; MP13/14). L'histoire des Theridomyoidea, avant leur première diversification constatée (MP14 ; e.g., Hartenberger, 1990), s'étend sur plusieurs millions d'années (entre $46 \mathrm{Ma}$ pour MP11/12 et 41 Ma pour MP14). Ainsi la différenciation du groupe apparaît plus progressive que constatée par Hartenberger sur la base de la documentation fossile d'alors. Par ailleurs, l'apparition du groupe n'est pas brutale au Lutétien, puisqu'on a pu proposer une origine européenne au sein d' « ischyromyoïdes » (Ischyromyiformes sensu Marivaux et al., 2004) de l'Eocène inférieur européen. En effet, Escarguel (1999) avait suggéré que les ischyromyoïdes les plus proches des Theridomyoidea étaient les genres Pantrogna Hartenberger (1971) et Hartenbergeromys Escarguel (1999). Nos analyses cladistiques (Marivaux et al., 2004 ; ce papier) confirment cette hypothèse. Ces deux genres constitueraient ainsi les premiers rameaux de la radiation initiale des Theridomorpha, même si leur foramen infra-orbitaire est encore protrogomorphe, quoique un peu élargi chez Hartenbergeromys (Escarguel, 1999).

\section{SYSTÉMATIQUE : ETAT DES LIEUX ET PROPOSI- TIONS}

Lavocat (1955) a proposé de reconnaître le sous-ordre des Theridomorpha. Si l'on doit maintenir ce rang, comment s'intègre-t-il alors à la classification des Rodentia en vigueur? Dans les vingt dernières années, la multiplication des données génétiques a permis de confirmer les liens super-familiaux ou familiaux des rongeurs mais sans pouvoir arriver à un consensus sur les relations infra-ordinales. Trois grands sous-clades de Rodentia sont bien identifiés : 1- le vaste « mouse-related clade » (incluant les Muroidea, Dipodoidea, Anomaluromorpha et Castorimorpha), 2- le « squirrelrelated clade " (incluant les Sciuridae et les Gliridae) et enfin 3- le clade Ctenohystrica (regroupant les Ctenodactylidae et les hystricognathes [Hystricidae, phiomorphes et caviomorphes] ; e.g., Huchon et al., 2000 ; Huchon \& Douzery, 2001; Huchon et al., 2007 ; Fabre et al., 2015). A la différence des informations génétiques, les données paléontologiques permettent d'envisager une dichotomie basale des Rodentia en séparant les Ctenohystrica d'un ensemble nommé Ischyromyiformes (Marivaux et al., 2004). C'est de ce dernier ensemble qu'émergeraient les " ischyromyioïdes ", les aplodontoïdes, les sciuroïdes, sciuravidés, castoroïdes (Eutypomys), c'està-dire les groupes-souches des Aplodontidae, Sciuridae, Gliridae, tous les " mouse-related » Cricetidae et Dipodidae et les theridomyoïdes. Nous adoptons ici cette hypothèse de dichotomie majeure précoce au sein de l'ordre des rongeurs, considérant deux sous-ordres principaux (sensu Marivaux et al., 2004) : les Ischyromyiformes et les Ctenohystrica. Au sein des Ischyromyiformes, les Theridomorpha se distinguent au rang d'infra-ordre.

Pour Lavocat (1955) et Thaler (1966), les Theridomorpha ne comprenaient que la super-famille des Theridomyoidea, tous ses membres étant hystricomorphes (i.e., possédant un large foramen infra-orbitaire) et sciurognathes ; leur formule dentaire la plus complète est $1 / 1 \mathrm{I}, 2 / 1 \mathrm{DP}, 1 / 1 \mathrm{P}, 3 / 3 \mathrm{M}$; si les plus anciens sont buno-lophodontes, beaucoup deviennent pentalophodontes (avec développement du mésolophe et du mésolophide dans la majorité des espèces) et les molaires supérieures sont quadrangulaires, pourvues d'un hypocône. Depuis, la définition des caractères diagnostiques de la denture des Theridomyoidea s'est affinée. En sus du développement de l'hypocône aux molaires supérieures, qui n'est pas d'emblée aussi gros que le protocône, des caractères de la denture inférieure se sont ajoutés. Il s'agit notamment de la postprotocristide longue et oblique, de l'ectolophide court, de la présence d'un entolophide et d'un hypoconulide réduit ou absent, étiré dans le postérolophide (e.g., Peláez-Campomanes, 1996 ; Vianey-liaud, 1998 ; Marivaux et al., 2004 ; Comte et al. 2012). Ces caractères des dents mandibulaires sont présents chez Hartenbergeromys et Pantrogna.

Hartenberger (1973b) n'a pas utilisé le sous-ordre Theridomorpha, la classification sub-ordinale des rongeurs s'avérant de « pratique difficile et loin d'avoir acquis le minimum de stabilité nécessaire ", seul le niveau superfamilial étant retenu. Hartenberger (e.g., 1971, 1973b) considérait aussi que la subdivision des Theridomyoidea en deux familles, Pseudosciuridae (Zittel, 1893) et Theridomyidae (Alston, 1876), n'était pas phylogénétiquement acceptable, du fait que ces deux familles lui apparaissaient polyphylétiques. Il s'appuyait sur le fait que la première incluait uniquement des formes à denture brachyodonte et, pour certaines, sélénodontes, tandis que la deuxième comprenait uniquement 
des espèces semi-hypsodontes. Cette division en deux familles a alors été abandonnée au profit de la reconnaissance de six sous-familles : les Pseudosciurinae, les Sciuroidinae, les Oltinomyinae, les Columbomyinae, les Theridomyinae et les Issiodoromyinae (Hartenberger, 1971 : fig. 2 et 1973b : figs. 7-9 ; Escarguel, 1998, 1999), ou bien maintenues mais avec un contenu révisé (e.g., Hooker, 1986 ; Hooker \& Weidmann, 2000). Escarguel a émis des doutes sur la position systématique des genres Protadelomys (Hartenberger, 1968) et Paradelomys (Thaler, 1966) au sein des Pseudosciuridae (sensu Hooker, 1986), ces genres étant pour cet auteur probablement des formes primitives de Theridomyidae. Adepte d'une division sous-familiale des Theridomyidae, unique famille retenue au sein des Theridomyoidea, il conserve les deux genres Protadelomys et Paradelomys provisoirement au sein de la sous-famille Sciuroidinae (Escarguel, 1998, 1999). Sur la base de quelques caractères dérivés, la famille des Pseudosciuridae est considérée comme monophylétique, distincte de la famille des Theridomyidae (Hooker, 1986 ; Hooker \&Weidmann, 2000 ; Comte et al., 2012) ; cette dernière pourrait inclure Protadelomys comme membre d'une famille non nommée (indet.) (Comte et al., 2012). La présente analyse, si elle confirme la pertinence d'une dichotomie Pseudosciuridae/ Theridomyidae, elle en écarte les Protadelomys ainsi que Tardenomys, qui constituent les divergences antérieures à cette dichotomie. Paradelomys apparaît alors comme le genre frère des Theridomyidae. Sur l'analyse réduite aux taxons éocènes (AE), Tardenomys se positionne après l'émergence des Pseudosciuridae, et il est étroitement apparenté à Paradelomys, à la base des Theridomyidae. La connaissance actuelle de Tardenomys (une seule espèce bien documentée) ne permet pas d'apporter des arguments pour l'une ou l'autre des propositions. Tardenomys est alors inclus provisoirement avec Protadelomys dans une même famille, non nommée (indet.), au sein des Theridomyoidea, tandis que Paradelomys est inclus dans une sous-famille indéterminée au sein des Theridomyidae.

\section{Sous-Ordre ISCHYROMYIFORMES Marivaux et al., 2004}

Ce sous-ordre englobe tous les Ischyromyoidea, Sciuroidea, Gliroidea, Muroidea, Dipodoidea, Geomyoidea, Castoroidea et Anomaluroidea ainsi que les Theridomorpha. Il n'est pas dans notre propos de discuter de leurs périmètres ni de leurs attributions infra-ordinales, excepté pour les Theridomorpha.

Les rémyoïdes ne sont plus considérés ici comme des Theridomorpha, cependant, l'état des connaissances les concernant est résumé dans ce chapitre. Ils sont présentés ici - au sein des Ischyromyiformes - comme le seront par la suite tous les taxons supra-génériques - au sein des Theridomorpha - (i- genres et espèces attribués ; ii- localités et niveaux biochronologiques, en référence aux niveaux repères mammaliens indiqués sur les figures 2 et 3 ; iiicaractères dentaires et, éventuellement, tendances évolutives et remarques ; iv- histoire paléogéographique). Lorsque le repérage biochronologique d'une localité ayant livré un taxon est incertain pour l'auteur de la description du taxon, le MP est précédé d'un «? »; il en est de même pour le signalement douteux d'un taxon dans une localité, le nom de la localité est précédé du signe «? ».
INFRA-ORDRE INDET.

\section{SUPER-FAMILLE REMYOIDEA nouveau rang}

Genres inclus. Zamoramys Peláez-Campomanes \& LópezMartinez, 1996 ; Santa Clara (Duero, Espagne), MP13/14, El Viso-Sanzoles (Duero, Espagne), MP14 ; + autres genres inclus dans les Remyidae.

Famille REMYIDAE Hartenberger, 1973, (rang in Quer \& Agusti, 2010)

Genre-type. Remys Thaler, 1966.

Genres et espèces inclus, localités et niveaux biochronologiques.

Frontyanamys russelli Quer \& Agusti, 2010, Frontyaná (Bassin de l'Ebre, Espagne), MP14-15.

Remys garimondi Thaler, 1966, Fons 1 (Gard, France), Malpérié (Tarn-et-Garonne, Quercy, France) MP17a ; Remys minimus Hartenberger, 1973b, Robiac, Fons 4 (Gard, France), Le Bretou (Tarn-et-Garonne, Quercy, France), Babilafuente (Duero, Espagne), MP16 ; Remys major Comte et al., 2012, Chéry-Chartreuve (Aisne, France), MP15, Les Alleveys (Suisse), MP15/16.

Pairomys crusafonti Thaler, 1966, San Cugat de Gavadons, ?Sossis, (Bassin de l'Ebre, Espagne), MP19, ?Zambrana (Miranda-Treviño-Basin, Alava, Pays Basque, Espagne), MP18 ; Pairomys ibericus Vianey-Liaud, SchmidtKittler \& Peláez-Campomanes, 1994, Mazateron, Miñana (Soria, Duero, Espagne) MP15/16-17 ?, Pairomys aff. ibericus, Babilafuente (Duero, Espagne) ? MP14/15.

Caractères dentaires (cf. Figs. 5, 10 et 11). Dents supérieures. $\mathrm{P} 4 \mathrm{~s}$ non molarisées, avec hypocône petit, plus lingual que le protocône et dépourvues de sinus. DP4s plus molarisées, avec abaissement de l'endolophe entre protocône et hypocône. Molaires : paracône et métacône à large base ; hypocône plus petit que le protocône et un peu plus lingual ; protolophe transverse et rectiligne; protoconule faible à absent; mésolophe absent à court, libre ou connecté au métaconule, métaconule présent bulbeux ou étiré, libre ou connecté à la préhypocrista.

Dents inférieures. $\mathrm{p} 4 \mathrm{~s}$ et surtout $\mathrm{dp} 4 \mathrm{~s}$ beaucoup plus petites que les molaires ; mésolophide absent ; antéroconide vestigial à absent ; métalophulide I comme un lophide mésial, séparé ou connecté à la préprotocrista ; mésolophide ébauché à l'extrémité linguale de la postprotocrista ou absent; entolophide rectiligne complet ou interrompu; hypoconulide présent.

Remarques. De Frontyanamys à Pairomys, on observe un accroissement asymétrique de la hauteur des couronnes, avec une élévation de l'endolophe aux molaires supérieures. En même temps, on note une simplification des lophides avec perte de l'ectolophide, tendance à l'interruption de l'entolophide orienté vers l'arrière et tendance à la bilophodontie chez Pairomys crusafonti.

Histoire paléogéographique. Cette famille apparaît d'abord en Espagne avec Frontyanamys (MP14/15) et peut-être Pairomys (? MP $14 / 15$, puis MP15/16 à 19) et se répand rapidement jusqu'au nord de le France et en Suisse (MP15/16 ; Remys). Elle est ensuite documentée dans le sud de la France (MP17) et en Espagne seulement, pour disparaître au cours du Priabonien (MP19, pour les derniers Pairomys). L'extension temporelle de la famille est d'environ $6 \mathrm{Ma}$. 


\section{INFRA-ORDRE THERIDOMORPHA Lavocat, 1955}

\section{Super Famille et Famille INDET.}

Genres inclus. Pantrogna Hartenberger, 1971 et Hartenbergeromys Escarguel, 1999.

Ces genres étaient inclus dans les Ischyromyidae, Reithoparamyinae, Microparamyini (e.g., Escarguel, 1999). Malgré leur parenté argumentée avec les Theridomyoidea, il n'est pas certain qu'ils puissent être intégrés au sein d'une division systématique formelle de cet infra-ordre avant que l'ensemble des espèces de Microparamyinae européens n'ait été révisé, notamment pour cerner le périmètre de cette division. En effet, Escarguel (1999 : 229-232) considère que le genre Masillamys est apparenté à ces deux genres, les trois étant bien séparés de Sparnacomys. Dans la présente analyse, Sparnacomys est plus basal mais étroitement apparenté à Pantrogna + Hartenbergeromys.

Nous considérerons ci-dessous les deux genres séparément, mais par la suite, pour tous les taxons de Theridomyoidea, la présentation sera plus synthétique (au niveau du genre et parfois de la sous-famille). Hartenbergeromys et Pantrogna ne sont pour l'instant documentés ni au Portugal ni en Espagne, mais toutes les faunes anciennes de rongeurs n'ont pas été étudiées.

\section{Genre Pantrogna Hartenberger, 1971b}

Espèce-type. Pantrogna russelli (Michaux, 1964) ; Avenay (Bassin-de-Paris, France), MP8-9.

Espèces incluses, localités et niveaux biochronologiques. Pantrogna russelli (Michaux, 1964), Avenay, Pourcy, Mutigny, Condé-en-Brie, St-Aignan (Bassin-de-Paris, France), MP8-9 ; P. marandati Escarguel, 1999, Prémontré (Bassin-de-Paris,
France), MP10 et Wittering Formation, Brackelsham Group (Sussex, Grande-Bretagne), MP10.

Caractères dentaires (Figs. 5, 10, 11 et 12). L'hypocône est présent sur les molaires supérieures et les DP4s, mais moins développé que le protocône ; l'endolophe est présent et affecté par un court sinus ; aux dents inférieures, les dp4s sont aussi longues que les p4s ; la postprotocristide est oblique buccodistalement ; l'ectolophide est court. Le foramen infra-orbitaire est petit et le palais est rectangulaire.

Histoire paléogéographique. Ces deux espèces sont connues de l'Eocène inférieur du Bassin-de-Paris et du sud de l'Angleterre seulement.

\section{Genre Hartenbergeromys Escarguel, 1999}

Espèce-type. Hartenbergeromys hautefeuillei Escarguel, 1999, Prémontré (Bassin-de-Paris, France), MP10.

Espèces incluses, localités et niveaux biochronologiques. Hartenbergeromys hautefeuillei Escarguel, 1999, St-Aignan et Grauves (Bassin-de-Paris, France), MP8-9 et MP10, Masde-Gimel, Naples et Azillanet (Languedoc, France) MP10 ; Hartenbergeromys parvus (Tobien, 1954), Messel (sud de l'Allemagne), MP11.

Caractères dentaires. Sur les molaires, l'endolophe, long mésio-distalement, est plus haut que chez Pantrogna. Bien que la dent soit quadrangulaire, l'hypocône est peu important, dans le prolongement de l'endolophe ; aux molaires inférieures, la partie buccale du métalophide s'attache à la pointe du protoconide ; elle rejoint parfois une ou deux crestules linguales issues du métaconide et distinctes de l'antérolophide.

Histoire paléogéographique. Ces deux espèces sont connues de l'Eocène inférieur du Bassin-de-Paris et du Languedoc et du début de l'Eocène moyen du sud de l'Allemagne.

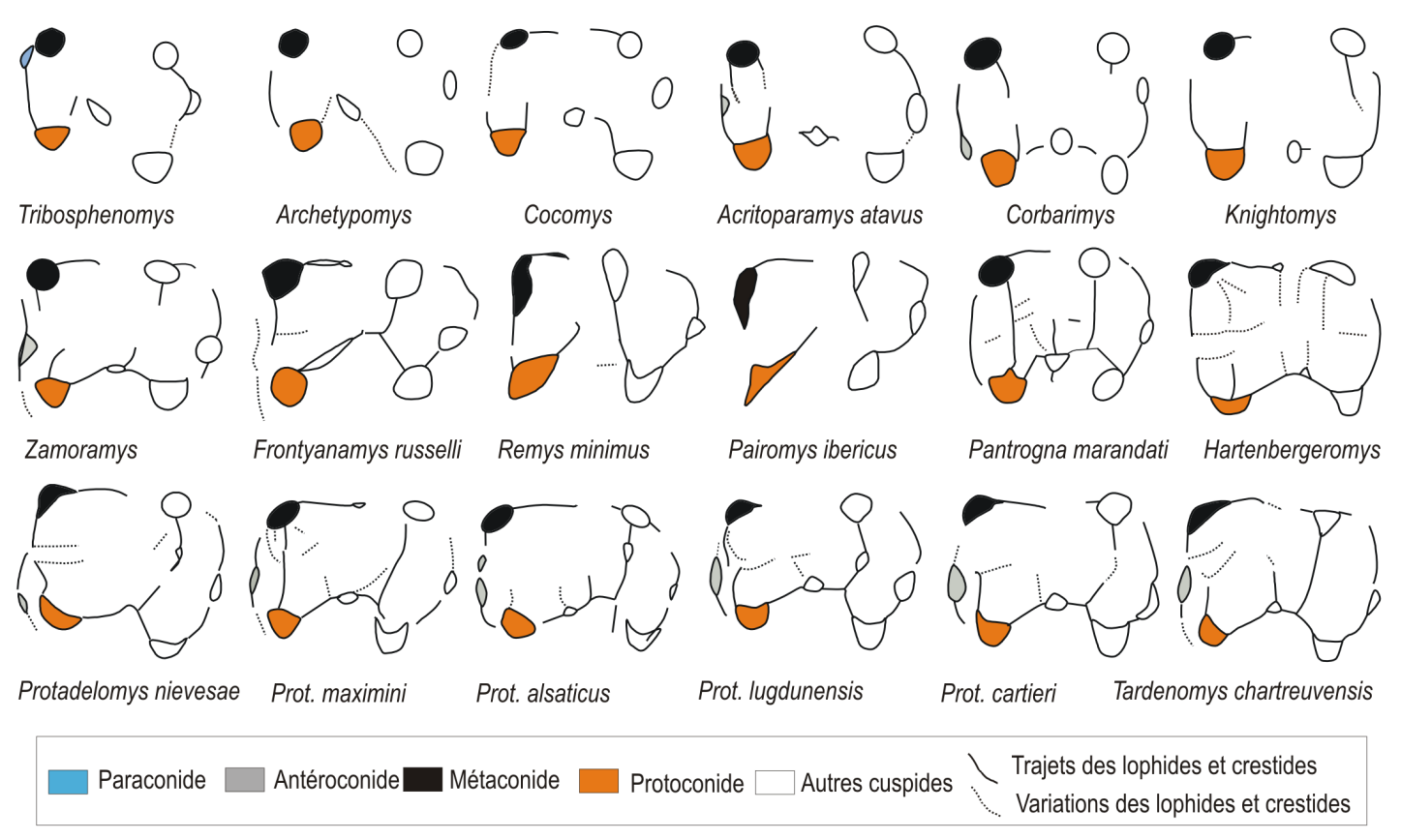

Figure 10. Schémas des m1-2s inférieures des diverses espèces des groupes externe et de branchement et des Remyoidea, comparées aux m1-2s des Theridomorpha basaux (Pantrogna et Hartenbergeromys : Theridomorpha indet.; Protadelomys et Tardenomys : Theridomyoidea indet.). 
SuPER-FAMILLE THERIDOMYOIDEA Lavocat, 1951

\section{FAMILLE INDET.}

Genres inclus. Protadelomys Hartenberger, 1968 et Tardenomys Comte et al., 2012.

Genre Protadelomys Hartenberger, 1968

Espèce-type. Protadelomys cartieri (Stehlin \& Schaub, 1951) ; Egerkingen $\gamma$ et Huppersand (Mormont, Jura Suisse), MP13/14.

Espèces incluses, localités et niveaux biochronologiques. Protadelomys cartieri (Stehlin \& Schaub, 1951), Egerkingen $\alpha$ et $\beta$, (Mormont, Jura Suisse), MP13-14;P. alsaticus Hartenberger, 1969, Bouxwiller (Alsace, France), MP13 ; P. lugdunensis Hartenberger, 1968, Lissieu (Rhône, France), MP14 ; P. nievesae Peláez-Campomanes, 1996, Casa Ramon (Soria, Espagne), MP 11/12 ; P. maximini Escarguel, 1998 (Saint-Maximin, Gard), un peu plus récent que MP 13.

Caractères dentaires. Theridomyoidea aux dents déciduales et prémolaires supérieures incomplètement molarisées (hypocône plus petit que le protocône, aligné avec l'hypocône ou légèrement lingual).

Molaires supérieures. Protocône plus ou moins allongé mésio-distalement, plus ou moins renflé, prolongé mésialement par une longue et oblique préprotocrista ; endolophe bas ou aussi élevé que le protocône, sinus absent ou présent et alors court, non comprimé ; présence variable du paraconule, toujours plus petit que le métaconule, qui peut être plus ou moins volumineux ; métacône au même niveau buccalement que le paracône, ou légèrement décalé lingualement ; métalophule I absent ou incomplet; métalophule II variablement connecté du postérocône au sommet du protocône ; mésolophe absent ou court; hypocône de moins à aussi développé que le protocône. La surface de l'émail est généralement ornementée, avec des granules et/ou des crêtes, au moins dans les bassins et aussi sur les flancs des cuspides et des lophes.

Molaires inférieures. Ectolophide court et mésio-distal ; composition de l'antérolophide simple (antéroconide) à complexe (antéroconide-antérocingulide + portion linguale du métalophulide I) ; métalophulide I complet ou divisé ; mésoconide renflé ou réduit ; mésolophide absent à court ; postprotocristide allongée, plus ou moins oblique ; hypoconulide plus ou moins bulbeux.

Histoire paléogéographique. Ce genre apparaît en Espagne (MP11/12), se disperse en France (MP13) et en Suisse (MP13-14). Sa dernière présence est signalée dans le nord de la France (MP15). L'extension temporelle du genre est de 5,5 Ma environ.

\section{Genre Tardenomys Comte et al., 2012}

Espèce-type. Tardenomys chartreuvensis Comte et al., 2012, Chéry-Chartreuve (Aisne, France), MP15.

Espèces incluses, localités et niveaux biochronologiques. Tardenomys chartreuvensis Comte et al., 2012, ChéryChartreuve (Aisne, France), MP15 ; Tardenomys sp., Les Alleveys (Mormont, Jura Suisse ; in Comte et al., 2012), MP15/16.

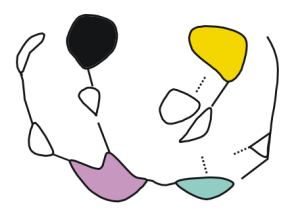

Frontanyamys russelli

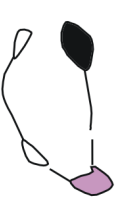

Remys minimus

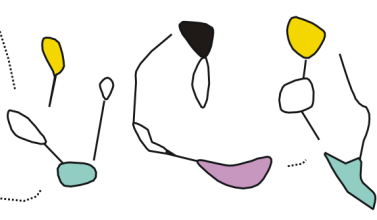

Pairomys ibericus

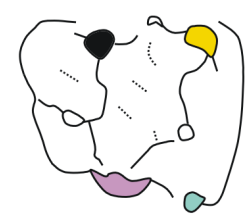

Pantrogna marandati Hartenbergeromys hautefeuillei

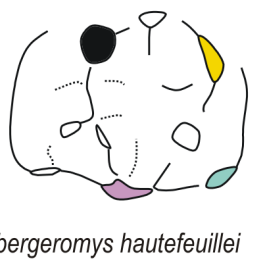

M1-2 sup.
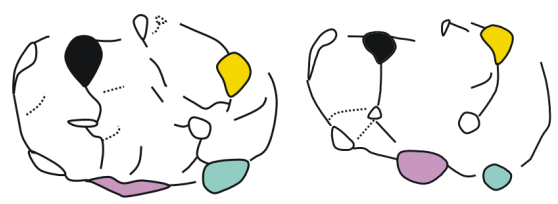

Protadelomys nievesae
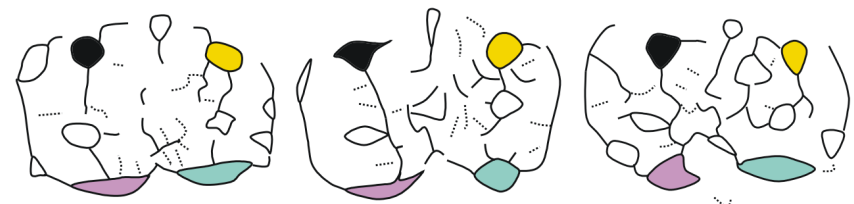

Treposciurus preceei

Treposciurus intermedius

Treposciurus manentis
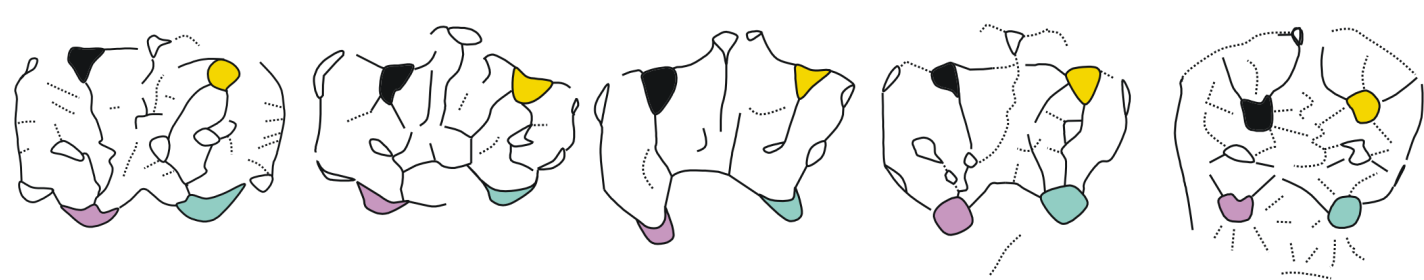

Sciuroides russelli

Tarnomys schmidt-kittleri

Tarnomys spelaeus

Suevosciurus ehingensis

Pseudosciurus suevicus

Paracône $\square$ Métacône

Protocône

Hypocône

Trajets des lophes et crestules Variations des lophes et crestules

Figure 11. Schémas des M1-2s supérieures de quelques Remyoidea, Theridomorpha (Hartenbergeromys et Pantrogna), Theridomyoidea (Protadelomys) basaux et Pseudosciuridae. 
Caractères dentaires (Figs. 10 et 12 ; Comte et al. 2012). Les granules et crestules ornementant la surface de l'émail dans les bassins sont plus nombreux chez Tardenomys que chez la plupart des Protadelomys. Les molaires supérieures diffèrent de celles de Protadelomys par le fort abaissement de l'endolophe et l'ébauche de la mure. Les molaires inférieures diffèrent de celles de Protadelomys par les lophides plus réguliers, le métaconide relativement moins élevé par rapport aux autres cuspides et la postprotocristide généralement plus longue.

Histoire paléogéographique. Ce genre est documenté dans le Bartonien basal du nord de la France (MP15) et de Suisse (MP15/16).

\section{FAmille PSEUDOSCIURIDAE Zittel, 1893}

Genre-type. Pseudosciurus Hensel, 1856.

Caractères dentaires. (Figs. 5, 11 et 13) Molaires supérieures. Mésostyle fort ; mésolophe court ou absent ; métalophe bucco- lingual généralement connecté à l'hypocône ; endolophe présent et sinus court; paraconule et métaconule généralement bulbeux à sommet plus ou moins appointé et aigu ; émail fréquemment ornementé (grossièrement à finement, formant des granules ou des crestules plus ou moins hauts ; ornementations isolées ou en réseau plus ou moins serré).

Molaires inférieures. Antérolophide distinct du métalophulide I, celui-ci étant souvent interrompu en son milieu (Sciuroides), incomplet (Pseudosciurus) ou complet et haut (Suevosciurus et Treposciurus). Attache buccale du métalophulide I avec le sommet du protocône.

Les genres sont ici répartis entre les deux sous-familles Pseudosciurinae et Sciuroidinae, suivant la dichotomie ressortant de l'analyse des taxons éocènes (AE).

Question de la "rétention des dents de lait" chez Suevosciurus. Les dents déciduales des Pseudosciuridae, notamment les DP4s, sont rarement décrites ou séparées des P4s dans le décompte des loci dentaires (e.g., Schmidt-

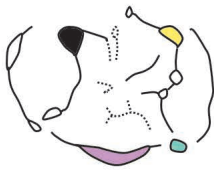

Protadelomys maximini Tardenomys chartreuvensis

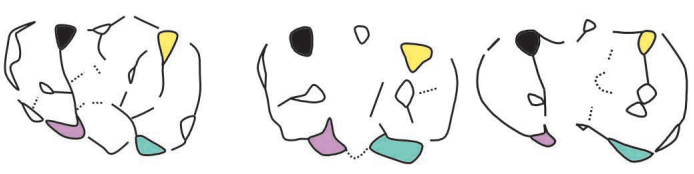

Elf. engesseri

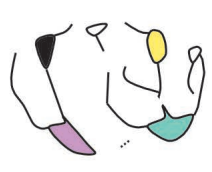

Elf. parvulus

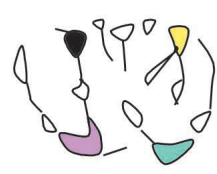

Pseudoltinomys crebrum

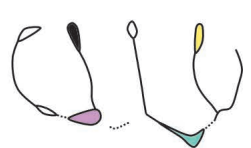

Pseudoltinomys gaillardi

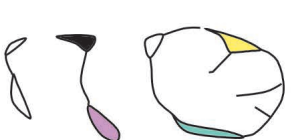

Issiodoromys bransatensis

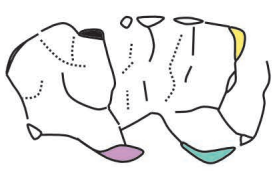

Thalerimys headonensis

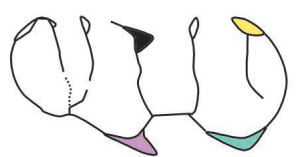

Theridomys. sp.(Ruch)

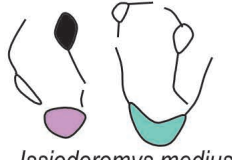

Issiodoromys medius

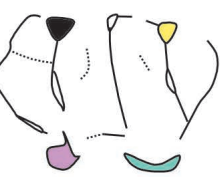

Oltinomys platyceps

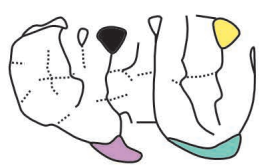

Thalerimys fordi

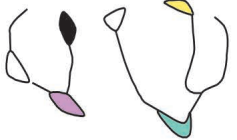

Issiodoromys minor

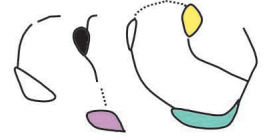

Issiodoromys pauffiensis

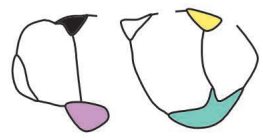

Issiodoromys limognensis

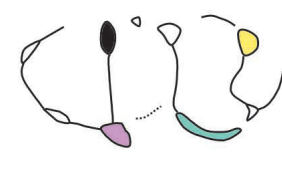

Patriotheridomys sudrei

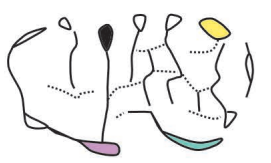

Thalerimys perrealensis

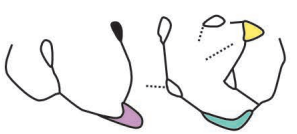

Patriotheridomys altus

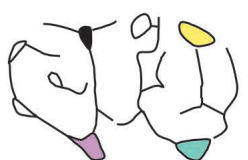

Theridomys golpei

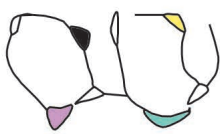

Sciuromys cayluxi

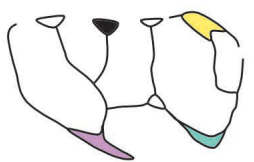

Theridomys calafensis

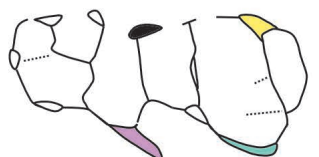

Theridomys major

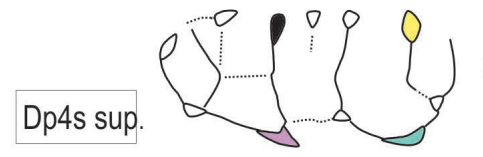

Paratheridomys margaritae

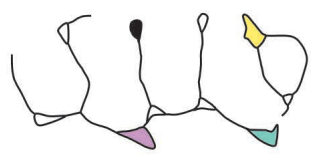

Paratheridomys ludensis

Paracône $\square$ Métacône $\square$ Protocône $\square$ Hypocône $\square$ Autres cuspides

Variations des lophes et crestules

Figure 12. Schémas des DP4s supérieures de quelques espèces de Theridomyidae. Comme pour les dents inférieures, les noms des espèces sont indiqués au dessous de chaque schéma. Il s'agit dans la plupart des cas de schémas réalisés à partir de spécimens de la population type, de moulages ou de figurations originales. Les cuspides sont schématisées par le contour de l'émail qui les borde, pris sur des dents peu usées. Les quatre cuspides principales sont colorées : en noir plein pour le paracône, en jaune pour le métacône, en violet pour le protocône et en vert pour l'hypocône. Les autres cuspides sont en formes vides (en blanc). Les trajets des crêtes et lophes sont schématisés par de simples traits pleins. Leurs variations et celles des crestules accessoires sont en traits pointillés. 
Kittler, 1971 : tabl.1). Hooker (1986) a émis l'hypothèse de la persistance des dents déciduales chez les individus adultes de Suevosciurus (i.e., absence de P4). Les DP4s de Suevosciurus authodon, $S$. bosmae, $S$. fraasi et $S$. ehingensis décrites par Hooker (1986) ont bien un prélobe constitué essentiellement du parastyle. Dans le matériel de $S$. authodon, on peut observer des DP4s ayant un parastyle saillant et un antérolophe réduit, mais il y a aussi de nombreuses P4s, sur lesquelles le parastyle est beaucoup moins protubérant, chez qui l'antérolophe est marqué bien que court, avec un antérostyle (ou antérostyle + paraconule vu sa position); le protocône et l'hypocône sont plus rapprochés que le paracône et le métacône.

Sur l'ensemble du matériel aujourd' hui disponible d'Escamps (Suevosciurus sp. dans cette analyse), huit spécimens dentaires restent ambigus, dans la mesure ou leurs caractères ne permettent pas de les attribuer de manière formelle à l'un ou l'autre des loci, DP4 ou P4. Toutefois, une dent, nettement plus étroite que les $\mathrm{M} 1 \mathrm{~s}$, pourrait dès lors correspondre à une DP4, car les autres spécimens (possibles P4s) sont en effet plus larges. Rappelons que les DP4s et P4s sont aussi longues que les M1s. Cette observation peut également s'appliquer aux spécimens attribués à l'espèce $S$. bosmae.

Chez les espèces oligocènes $S$. fraasi et $S$. ehingensis les dents déterminées antérieurement comme $\mathrm{P} 4 \mathrm{~s}$ sont considérées par Hooker (1986) comme des DP4s, i.e., non remplacées. Scherzinger et al. (2004) continuent cependant à présenter ces dents mésiales comme des $\mathrm{P} 4 \mathrm{~s}$. Bien que ces déterminations soient plausibles, certaines zones d'ombre persistent : il reste à établir formellement les critères morphologiques séparant les P4s et DP4s dans le matériel allemand de $S$. fraasi et de $S$. ehingensis. Or, il y a très peu de matériel dans chaque localité (e.g., Schmidt-Kittler, 1971, p.46 : maximum neuf à Ehrenstein $1 \mathrm{~A}$ et sept à Weissenburg 8 pour $S$. fraasi ; sinon, pour les sept autres localités, leur nombre est compris entre 1 et 4 . Le nombre de spécimens est même inférieur pour $S$. ehingensis : huit au maximum à Herrlingen 1 et entre un et deux dans les cinq autres localités listées). Le matériel est beaucoup plus abondant pour les deux espèces de Pseudosciurus. Aussi, les DP4s et les P4s ont pu y être identifiées avec certitude (Ps. suevicus : 109 à Herrlingen 1, 80 à Ehingen 14, 50 à Ronheim $1+60$ autres dans 14 localités).

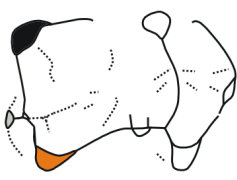

Sciuroides rissonei

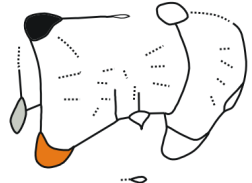

Sciuroides russelli

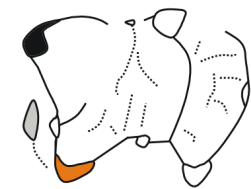

Sciuroides romani

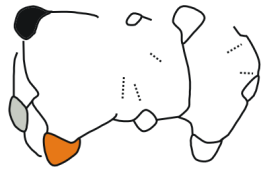

Sciuroides ehrensteinensis

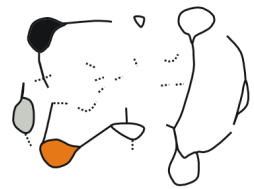

Sciuroides siderolithicus

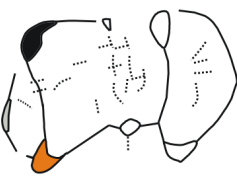

Tarnomys helveticus

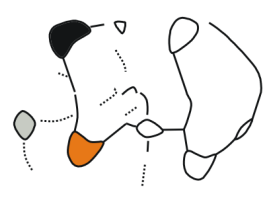

Tarnomys schmidt-kittleri

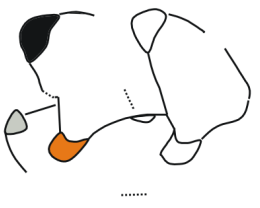

Tarnomys spelaeus

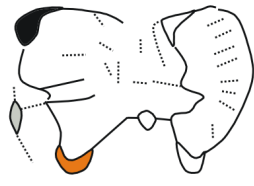

Tarnomys quercinus

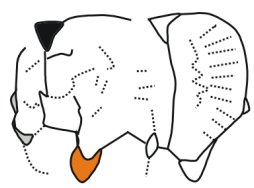

Pseudosciurus precedens

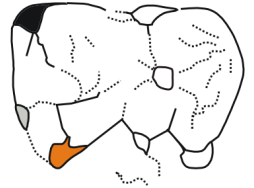

Pseudosciurus suevicus

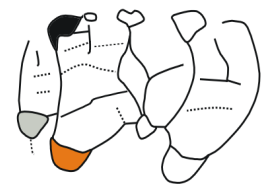

Treposciurus manentis

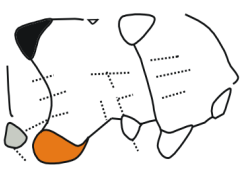

Treposciurus gardneri

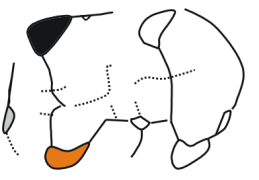

Treposciurus preceei

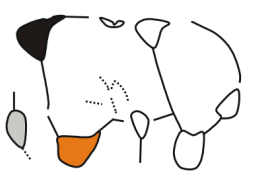

Treposciurus intermedius

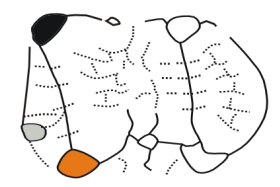

Treposciurus mutabilis

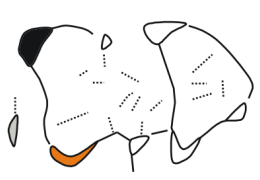

Suevosciurus authodon

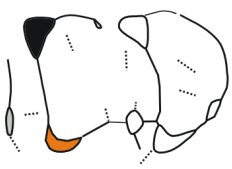

Suevosciurus bosmae

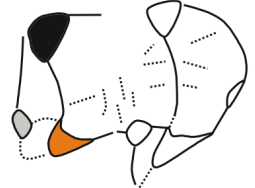

Suevosciurus minimus

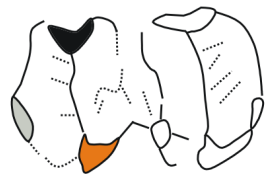

Suevosciurus ehingensis

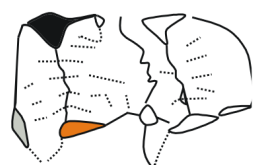

Suevosciurus fraasi

Antéroconide Métaconide

Protoconide $\square$ Autres cuspides

Trajets des lophides et crestides

Variations des lophides et crestides

Figure 13. Schémas des m1-2s inférieures de diverses espèces de Pseudosciuridae. 
Il est donc hautement vraisemblable que le caractère « rétention de la DP4 chez Suevosciurus » résulte d'un biais d'observation.

\section{Sous-famille Pseudosciurinae Zittel, 1893}

Genre-type : Pseudosciurus Hensel, 1856.

Espèce-type. Pseudosciurus suevicus Hensel, 1856 (Jura Souabe, Allemagne).

\section{Genres et espèces inclus, localités et niveaux} biochronologiques.

Pseudosciurus procedens Schmidt-Kittler, 1971, Ehrenstein 1, Weissenburg 8, Mähringen, Huisheim, Herlingen 3 (Jura Souabe, Allemagne), MP18-MP19 ; Pseudosciurus suevicus Hensel, 1856, Liptingen 1, 8 et loc. div. (Jura Souabe, Allemagne : cf. Schmidt-Kittler, 1971 : tab.1, p.15), MP19-22.

Tarnomys quercinus Hartenberger \& Schmidt-Kittler, 1976, Ravet (Tarn-et-Garonne, Quercy, France) MP21, Aguatón (Teruel, Espagne), MP21 ; Tarnomys schmidtkittleri Hooker \& Weidmann, 2000, Entreroches, Eclépens-Gare, (Mormont, Jura Suisse), MP15/16-19 ; Tarnomys quercyi (Schlosser, 1884), Eclépens C (Mormont, Suisse), Escamps et ?SteNéboule (Lot, Quercy, France), ?Hatherwood (Angleterre), MP18-19 ; Tarnomys helveticus (Schmidt-Kittler, 1971), Eclepens B (Mormont, Jura Suisse), MP17 ; Tarnomys spelceus (Hartenberger, 1973b), Escamps, (Lot, Quercy, France), MP19; Tarnomys aff. spelceus, La-Débruge (Vaucluse, France), MP18.

Caractères dentaires. Cuspides élevées et coniques ; sélénodontie ébauchée ou complète (Pseudosciurus) ; surface de l'émail à ornementation de moyennement à fortement ridulée, parfois réticulée.

Dents supérieures. Mésolophe grêle et parfois incomplet chez Tarnomys, réduit chez Pseudosciurus et fusionné dans les nombreuses crestules accessoires ; paraconule et métaconule présents, plus ou moins isolés ; protolophe et métalophule s'abaissant et/ou interrompus au centre de la dent, très incomplets chez Pseudosciurus ; métalophule I réduit, éventuellement connecté à l'extrémité mésiale de la préhypocrista ; mure ébauchée, incomplète ou complète ; endolophe vestigial ou absent.

Dents inférieures. Métalophulide I sinueux, parties buccale et linguale disjointes sur les dents peu usées au moins ; entolophide complet et de hauteur irrégulière, ou s'interrompant à la jonction avec la préhypocristide, très incomplet chez Pseudosciurus suevicus où un entoconulide fort est développé ; mésoconide présent avec court ectomésolophide mais mésolophide absent.

Histoire paléogéographique. Cette sous-famille est d'abord signalée en Suisse dès l'Eocène moyen et gagne l'Allemagne du sud, le nord de la France et le sud de l'Angleterre au début du Bartonien (MP15), puis le sud de la France (MP17). Les espèces de Tarnomys ont une répartition de la Suisse à l'Espagne au Bartonien terminal/Priabonien basal (MP17), pour occuper toute l'aire de répartition au cours du Priabonien (jusqu'à MP19-20). Elles se limitent essentiellement au nord de l'Espagne et au sud de la France durant les derniers temps de leur histoire évolutive (MP21). Les différentes espèces de Pseudosciurus, chez qui la sélénodontie est la plus développée, se succédent dans le temps et évoluent seulement au sud de l'Allemagne (MP18 à MP22). L'extension temporelle de la sous-famille est d'environ $8 \mathrm{Ma}$.
Sous-famille ScIUROIDINAE Hartenberger, 1971a

Espèce-type. Sciuroides siderolithicus (Pictet \& Humbert, 1869), Eclepens-Gare (Mormont, Jura Suisse), MP16/17.

Genres et espèces inclus, localités et niveaux biochronologiques.

Sciuroides siderolithicus (Pictet \& Humbert, 1869), Les Alleveys, (Mormont, Jura Suisse), MP15/16 ; Sciuroides ehrensteinensis Schmidt-Kittler, 1971, Ehrenstein 1A (Allemagne du sud), MP18; Headon Hill HH1-4 et Totland Bay (Ile de Wight, Angleterre), MP18 ; Sciuroides russelli (Hartenberger \& Louis, 1976), Grisolles et Rocourt-SaintMartin (Aisne, France), MP16 ; Sciuroides rissonei Hooker, 1986, Creechbarrow (Hampshire, Angleterre), MP16 ; Sciuroides romani (Hartenberger, 1973b), Robiac (Gard, France), MP16.

Treposciurus sp. Comte et al., 2012, Chéry-Chartreuve (Aisne, France), MP15 ; Treposciurus mutabilis (SchmidtKittler, 1971), Ehrenstein 1 (Jura Souabe, Allemagne), MP18, St-Lizier (Tarn, France), MP16 ; A.C. Quercy (France), niveau indet. ; Treposciurus intermedius (Schlosser, 1884), Ehrenstein 1 (Jura Souabe, Allemagne), MP18, St-Lizier (Tarn, France), MP16 ; A.C. Quercy (France), niveau indet. ; ?Sossis (Bassin de l'Ebre, Espagne), MP17 ; Treposciurus sp., in Hartenberger (1969 : pl. 1, fig. 4) Egerkingen $\gamma$ (Mormont, Jura Suisse), MP13/14 ; Treposciurus preceei Hooker, 1986, Creechbarrow (Hampshire, Angleterre), MP16 ; Treposciurus gardneri Hooker, 1991, Entreroches (Mormont, Jura Suisse), Headon Hill : HH1-HH4 (Ile de Wight, Angleterre), MP18-20; Treposciurus manentis Peláez-Campomanes, 2000, Aguatón (Teruel, Espagne), MP21.

Suevosciurus sp. Comte et al., 2012, Chéry-Chartreuve (Aisne, France), MP15/16 ; Suevosciurus authodon Hooker, 1986, Creechbarrow (Hampshire, Angleterre), MP16 ; Suevosciurus minimus (Major, 1873), Eclépens (Mormont, Jura Suisse), MP17, A.C. Quercy (France), niveau indet. ; Suevosciurus sp., Escamps (Lot, Quercy, France), MP19 ; Suevosciurus ehingensis Dehm, 1937, Ehingen 1 et loc. div. (Jura Souabe, Allemagne), MP19-25, Detán (Bohème, République tchèque) (Fejfar, 1987), MP21, Armissan (Aude, France), Itardies (Lot, Quercy, France) MP23 ; Suevosciurus fraasi (Major, 1873), Ehrenstein1, Grafenmühle 6A-B,10, Weissenburg 8, Oppertshofen 2, Möhren 6, 7/16, 13, 19, 20, 31, Herrlingen 1, Schelklingen 1, Liptingen 2, 8, 12 (Jura Souabe, Allemagne), MP18-23; Suevosciurus dehmi Heissig, 1987, Weissenburg 8, Oppertshofen 2, Möhren 19, Haag 2, Burgmagerbein 3/5/8, Grafenmühle 6B,10 (Jura Souabe, Allemagne), MP19-22 ; Suevosciurus bosmce Hooker, 1991, Headon Hill : HH1, HH3 (Ile de Wight, Angleterre), MP18 ; Suevosciurus palustris (Misonne, 1957), Hoogbutsel (Belgique), MP21.

Caractères dentaires. Dents bunodontes à bunolophodontes. Granules, ridules et crestules variablement développées, généralement peu élevées, dans les bassins ou le long des lophes-lophides.

Dents supérieures. Parties linguales des métalophule I et II convergent vers ou connectées à l'hypocône ; conules modérément développés à réduits ; mésolophe court ou absent ; endolophe présent généralement fort ; sinus court et souvent étroit; mure absente.

Dents inférieures. Antéroconide en position buccale ; antérolophide bas (= cingulide) et distinct du métalophulide 1 ; métalophulide 1 complet rarement interrompu entre ses 
parties linguale et buccale ; ectolophide relativement long ; entolophide complet ; mésoconide présent, mésolophide et ectomésolophide réduits à absents.

Histoire paléogéographique. Le genre Sciuroides est présent seulement du Bartonien (MP15-16) au Priabonien (MP17-18), en Suisse, en France - du nord au sud - et en Angleterre. Les autres genres occupent le même territoire, étendu à la Belgique, l'Espagne et l'Allemagne du sud, mais sur une plus longue période de temps. Notamment «Treposciurus » manentis est documentée au début de l'Oligocène (MP21) en Espagne, tandis que les lignées de Suevosciurus occupent toute l'aire de répartition jusqu'à la fin du Priabonien (MP20), puis le genre est signalé dans le sud de la France, en Belgique, en République tchèque et Allemagne au début du Rupélien (MP21-23). L'aire est restreinte à l'Allemagne du sud jusqu'au début du Chattien (MP25). La sous-famille a une extension temporelle de 13,5 Ma.

\section{FAMILLE THERIDOMYIDAE Alston, 1876}

\section{Sous-famille INDET. 1}

\section{Genre Paradelomys Thaler, 1966}

Espèce-Type. Paradelomys crusafonti Thaler, 1966, Sossis (Bassin de l'Ebre, Espagne), MP16.

Espèces incluses, localités et niveaux biochronologiques. Paradelomys santjaumensis Bonilla-Salomón et al., 2016, Sant-Jaume-de-Frontyaná (Espagne), MP15 ; Paradelomys crusafonti Thaler, 1966, Sossis (Bassin de 1'Ebre, Espagne), Robiac, Euzet, Fons 4 (Gard, France), Le Bretou, Malpérié (Tarn-et-Garonne, Quercy, France), Rocourt-St-Martin, Grisolles (Aisne, France), Eclépens B (Mormont, Jura Suisse), St-Lizier (Tarn, France), MP16-17 ; Paradelomys ruetimeyeri (Pictet \& Humbert, 1869), Entreroches (Mormont, Jura Suisse), MP19.

Caractères dentaires. (Figs. 5, 14 et 15) Dents faiblement semi-hypsodontes.

Dents supérieures. Protolophe et métalophe souvent interrompus lingualement; endolophe réduit à absent ; mure absente à ébauchée ; métalophule I présent à réduit ; conules présents, bien développés à réduits ; mésolophe et mésolophule courts.

Dents inférieures. Antésinuside et cingulide buccal présents, métalophide I connecté au sommet du protoconide ; préhypocristide oblique, longue et en continuité avec l'ectolophide court, de mésio-distal à oblique ; mésolophide absent ; entolophide complet et bas.

Remarques. De $P$. santjaumensis à $P$. crusafonti, on peut constater une augmentation de la taille de l'hypocône, la rupture de l'endolophe et l'ébauche de la mure, la diminution des conules, la réduction du métalophule I et le métalophule II s'orientant souvent vers le postérolophe. Aux molaires inférieures les granules disparaissent de la surface de l'émail, les lophides sont plus élevés et complets et le sinuside est plus enfoncé bucco-lingualement.

Histoire paléogéographique. Ce genre apparaît au nord de l'Espagne (MP15), puis se répand du sud de la France au Jura Suisse (MP16-17), aire sur laquelle il subsiste à la fin du Priabonien (MP19). L'extension temporelle du genre est d'environ 5,5 Ma.
Sous-famille IssiodoromyinAE Tullberg, 1899

Genre-type. Issiodoromys Croizet, in Gervais, 1848.

Genres et espèces inclus, localités et niveaux biochronologiques.

Elfomys Hartenberger, 1971 : Elfomys tobieni (Thaler, 1966), Robiac (Gard, France), Rocourt-Saint-Martin (Aisne, France), MP16, Fons 4 (Gard, France), MP17 ; Elfomys engesseri Hooker \& Weidmann, 2007, Les Alleveys (Mormont, Jura Suisse), MP15/16, Chéry-Chartreuve (Aisne, France), MP15/16 ; Elfomys parvulus (Hartenberger, 1971a), ?Sossis (Bassin de l'Ebre, Espagne), MP17, Gousnat (Tarn-et-Garonne, Quercy, France), MP18, Euzet (Gard, France), Perrière et Malpérié (Tarn-et-Garonne, Quercy, France), MP17.

Issiodoromys Croizet in Gervais, 1848 : Issiodoromys hartenbergeri (Vianey-Liaud \& Ringeade, 1993), Lascours (Gard, France), Escamps (Lot, Quercy, France), MP18b MP19 ; Issiodoromys nanus (Thaler, 1969), Montalbán 1, 7, 10, 11, 12 (Teruel, Espagne), MP23 ; Issiodoromys medius (Vianey-Liaud, 1976), Villebramar (Lot-et-Garonne, France), MP21, Mas-de-Got B, Pech-Crabit, La Plante 2, Baraval (in Sigé et al., 1998 ; Lot, Quercy, France), Balm (Suisse), Les Chapelins (Vaucluse, France), Valdecollares (Cuenca, Espagne), MP22-MP23 ; Issiodoromys minor Schlosser, 1884, Lébratières 14, Belgarric 1, Garouillas, Rigal-Jouet 1, (Tarnet-Garonne, Quercy, France), Vialenc (Cantal, France), StMartin-de-Castillon (Vaucluse, France), Grenchen 1, Talent 7, (Molasse Suisse), Olalla 4 - 10, Montalbán 1, 3, 8, 7, 9, 10, Mirambueno 3, 4B, 15 (Teruel, Espagne), Carrascosa-delCampo (Cuenca, Espagne), Pareja (Espagne), MP24 et MP25 ; Issiodoromys pauffiensis Vianey-Liaud, 1976, Mas-de-Pauffié, La-Devèze et Espeyrasse (Lot, Quercy, France), Puycelsi (Tarn, France), Les-Milles (Bouches-du-Rhône, France), SaintPrivat-des-Vieux (Gard, France), Oensingen, MümliswylHardberg, (Molasse Suisse), Mirambueno 4C-4D (Teruel, Espagne), MP26 ; Issiodoromys quercyi Schlosser, 1884, A.C. Quercy niveau indet., Sarèle, Boujac, (Gard, France), Mirambueno 1-2A-2B-2C, Barranco de Oceca 2C, Salobral 2, Villanueva del Rebollar 2-3-4 (Teruel, Espagne), Wynau 1, Boningen (Molasse Suisse), Ehrenstein7, Gaimersheim2, Burgmagerbein1 (Jura Souabe, Allemagne), MP27 ; Issiodoromys limognensis Vianey-Liaud, 1976, Pech-Desse, Pech-du-Fraysse, (Lot, Quercy, France), Vivel del Rio (Teruel, Espagne), Canales (Loranca, Espagne), Fornant 6-7 (Molasse Suisse), Gaimersheim1 (Jura Souabe, Allemagne), MP28 ; Issiodoromys pseudanaema Gervais, 1848, Rickenbach, Nant d'Avril, Rances, Fluh 19/20 (Molasse Suisse), Parrales (Loranca, Espagne), St Victor-la-Coste (Gard, France), MP29 ; Issiodoromys bransatensis Hugueney, 1969, Coderet (Allier, France), Thézels (Lot-et-Garonne, France), Venelles (Bouchesdu-Rhône, France), Ehrenstein 4 (Jura Souabe, Allemagne), MP30.

Pseudoltinomys Lavocat, 1951 : Pseudoltinomys sp., ChéryChartreuve (Aisne, France), MP15/16; Pseudoltinomys crebrum Peláez-Campomanes, 1996, Mazateron (Soria, Espagne), Les Alleveys (Suisse), MP15/16/?17; Pseudoltinomys mamertensis Hartenberger, 1973b, Fons 4 et ?Euzet (Gard, France), Le Bretou (Tarn-et-Garonne, Quercy, France), St-Lizier (Tarn, France), MP16-17 ; Pseudoltinomys phosphoricus Hartenberger, 1973b, Perrière, Malpérié (Tarn-et-Garonne, Quercy, France), Sossis (Huesca, Espagne), MP17 ; Pseudoltinomys gousnatensis Vianey-Liaud, 1976, Gousnat (Tarn-et-Garonne, Quercy, France), MP18a ; Pseudoltinomys cosetanus Hartenberger, in 
Anadon et al., 1987, Pontils 26 (Bassin de l'Ebre, Espagne), MP16 ; Pseudoltinomys amblesi Garzón Heydt \& López Martinez, 1978, Los Barros (Avila, Espagne), MP22 ou 23 ; Pseudoltinomys cuvieri (Pomel, 1852-3), Montmartre (Paris, France), Sainte Néboule, Escamps, Rosières 2-3, (Lot, Quercy, France), Civrac (Gironde, France), Saint-Capraise-d'Eymet (Dordogne, France), San-Cugat-de-Gavadons (Bassin de
1'Ebre, Espagne), MP18 à MP20 ; Pseudoltinomys gaillardi Lavocat, 1951, Ronzon (Haute-Loire, France), Aubrelong1, Ravet (Lot, Quercy, France), Mazan, Fontaines-de-Vaucluse (Vaucluse, France), Soumailles (Aquitaine, France), MP21 ; Pseudoltinomys major Vianey-Liaud, 1976, Villebramar (Lot-et-Garonne), La Plante 2, Mas-de-Got A, Itardies, Roqueprune 2, Pech-Crabit, Coulou, Mounayne, Baraval (Lot,
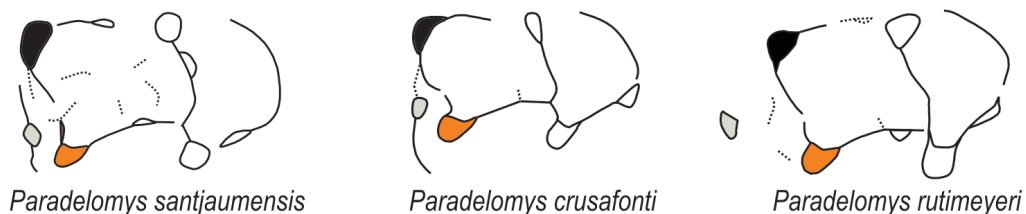

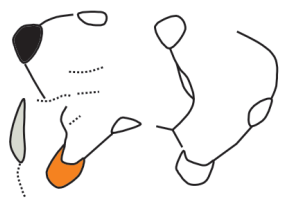

Elfomys catalaunicus

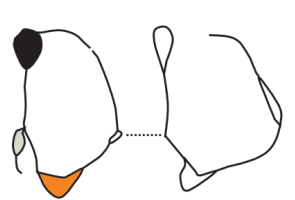

Issiodoromys hartenbergeri

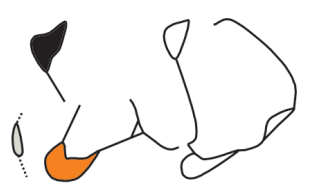

Pseudoltinomys crebrum

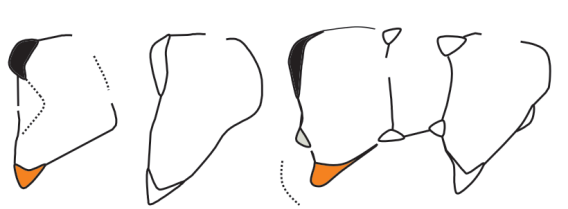

Bernardia marandati Patriotheridomys neboulensis

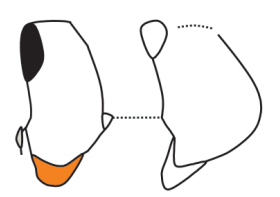

Issiodoromys medius

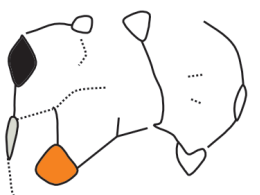

Ps. cosetanus

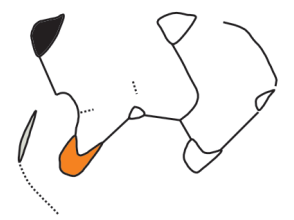

Elfomys engesseri

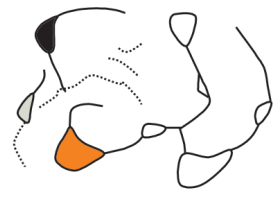

Elfomys tobieni

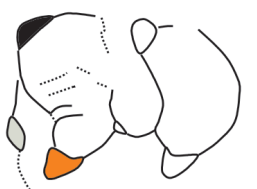

Elfomys parvulus

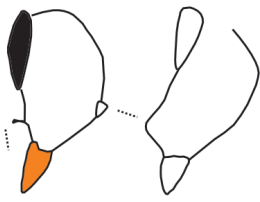

Issiodoromys minor

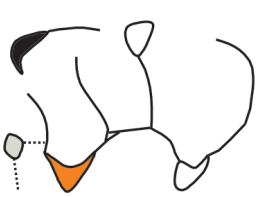

Ps. phosphoricus

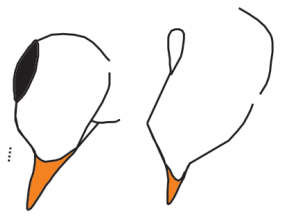

Issiodoromys pauffiensis

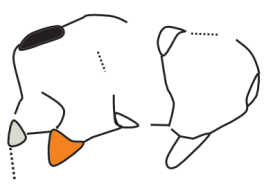

Ps. mamertensis

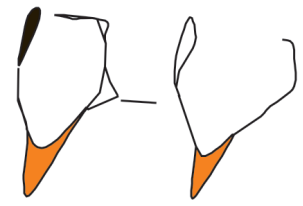

Issiodoromys limognensis

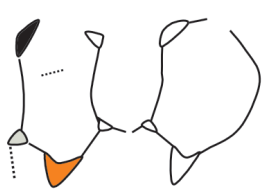

Ps. cuvieri

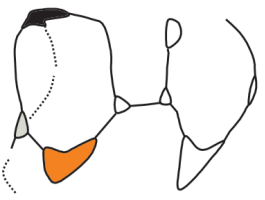

Patriotheridomys altus

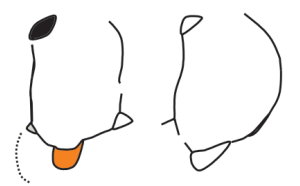

Ps. gaillardi

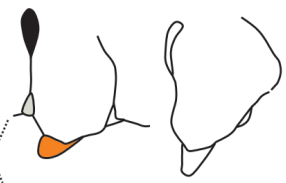

Ps. major

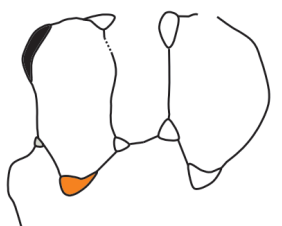

Ectropomys exiguus

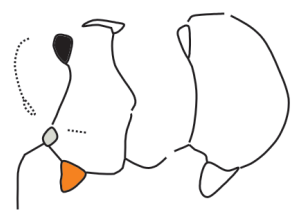

Oltinomys platyceps

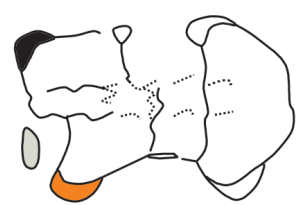

Estellomys cansouni

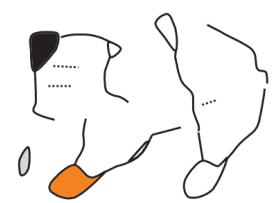

Estellomys varleti

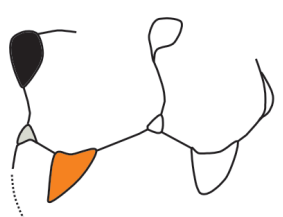

Sciuromys cayluxi

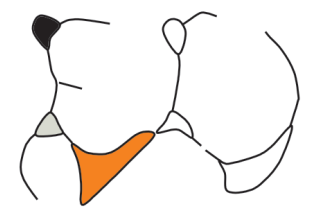

Sciuromys rigali

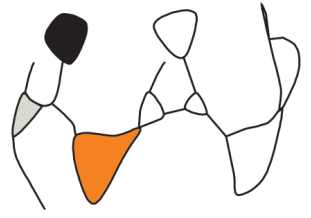

Columbomys cuencae

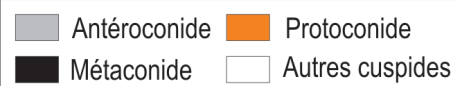

Trajets des lophides et crestides

Variations des lophides et crestides

Figure 14. Schémas des m1-2s inférieures de diverses espèces de Theridomyidae : sous-famille indet. (Paradelomys et Estellomys); Issiodoromyinae (Elfomys, Pseudoltinomys et Issiodoromys); Patriotheridomyinae (Bernardia et Patriotheridomys) ; Oltinomyinae (Ectropomys et Oltinomys) ; Columbomyinae (Sciuromys et Columbomys). 
Quercy, France), Les Chapelins (Vaucluse, France), Montalbán (Teruel, Espagne), MP22-23.

Caractères dentaires. (Figs. 5, 12, 14 et 15) Les prémolaires sont plus petites que les molaires dans les trois genres ( $\mathrm{L}: \mathrm{p} 4$ $<\mathrm{m} 1<\mathrm{m} 2<\mathrm{m} 3$; L : P4 < M1 < M2 > M3). C'est seulement chez l'espèce ultime de Pseudoltinomys (P. major) que les $\mathrm{p} 4 \mathrm{~s}$ inférieures sont aussi longues, voire plus, que les $\mathrm{m} 1 \mathrm{~s}$.

Dents supérieures. Interruption profonde de l'endolophe entre protocône et hypocône, d'où connexion entre sinus et synclinal opposé (SII) ; métalophe court, libre lingualement et/ ou connecté au postérolophe.

Dents inférieures. Interruption peu profonde de l'ectolophide; entolophide complet; antésinuside moyennement long à court et profond ; sinuside asymétrique ; mésolophide d'absent à long; postmétacristide longue, tendant à clore lingualement le premier synclinide du talonide lorsque le mésolophide est long.

Remarques. Tendances évolutives. Couronne bunolophodonte à lophodonte, avec aplanissement de la surface d'usure ; allongement des dents déciduales chez Issiodoromys seulement, de légèrement à fortement plus longues que les prémolaires, avec développement de crêtes additionelles antérieures à partir des espèces oligocènes ; réduction à disparition des conules ; mésolophe et mésolophide complets, depuis les espèces priaboniennes ; accroissement de la semihypsodontie depuis les espèces bartoniennes (MP15-MP16), jusqu'à l'hypsélodontie (croissance très prolongée à continue, réduction jusqu'à la perte des racines) chez les espèces de la fin de l'Oligocène ; antéroconide et antésinuside réduits, disparaissant progressivement avec l'accroissement de l'hypsodontie ; allongement transverse des sinuside et sinus, disparition des synclinaux et synclinides (du fait qu'ils ne se développent pas en hauteur comme le fût de la couronne) et aplanissement des surfaces d'usure.

Histoire paléogéographique. Cette famille est répartie de l'Espagne à l'Ile de Wight jusqu'à la molasse Suisse, et en France, du Bartonien (MP14-15) jusqu'à leur extinction, à la fin de l'Oligocène (MP30). Le genre Issiodoromys atteint le sud de l'Allemagne seulement à la limite Rupélien/Chattien (MP24) ; il y demeure jusqu'à la fin de l'Oligocène (MP30). La sous-famille s'étend sur $18 \mathrm{Ma}$, le genre Issiodoromys sur 12,5 Ma.

\section{Sous-famille OLtinomyinae Hartenberger, 1971}

Genre-type. Oltinomys platyceps (Filhol, 1877).

Genres et espèces inclus, localités et niveaux biochronologiques.

Oltinomys platyceps (Filhol, 1877), A.C. Quercy (France), Escamps, Sindou D, Rosières 2, Tabarly (Lot, Quercy, France), MP19-20.

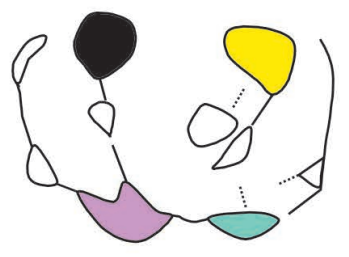

Frontanyamys russelli

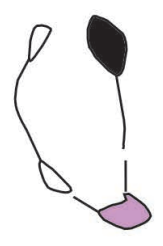

Remys minimus

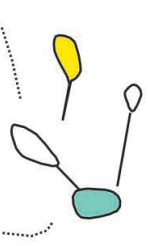

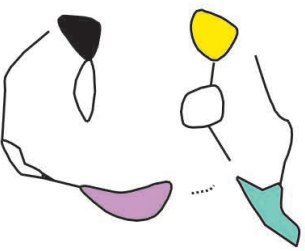

Pairomys ibericus

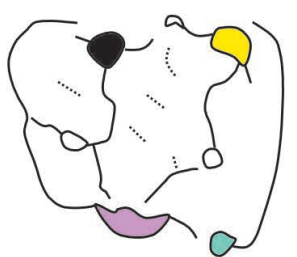

Pantrogna marandati Hartenbergeromys hautefeuillei

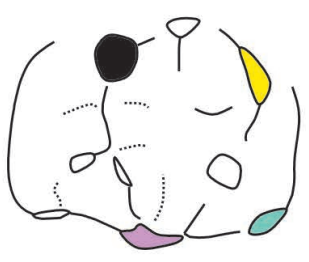

M1-2s sup.
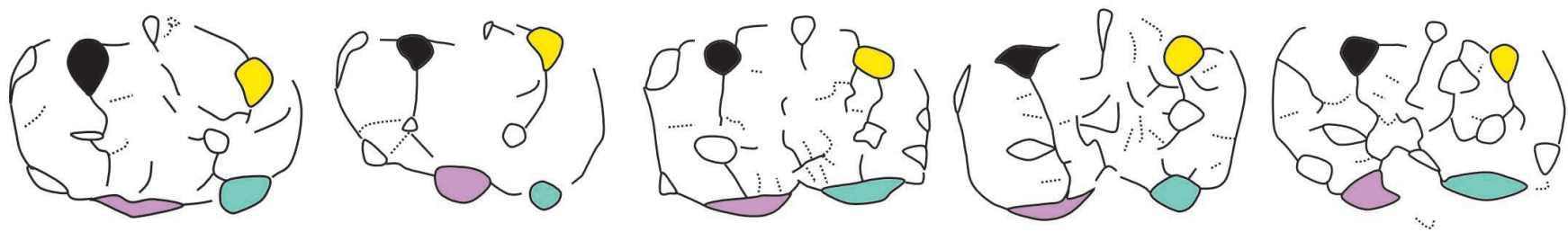

Treposciurus preceei

Treposciurus intermedius

Treposciurus manentis
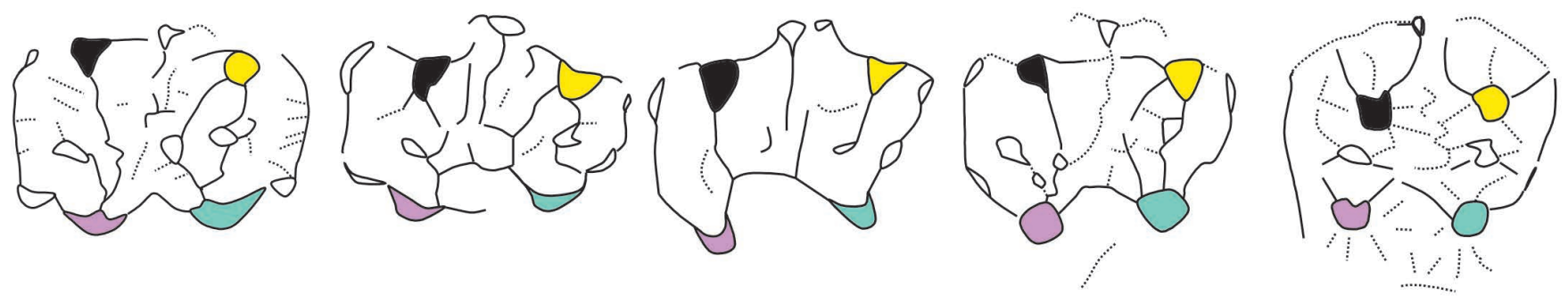

Suevosciurus ehingensis

Pseudosciurus suevicus

Sciuroides russelli

Tarnomys schmidt-kittleri

Tarnomys spelaeus

Trajets des lophes et crestules Variations des lophes et crestules 
Ectropomys exiguus Bosma \& Schmidt-Kittler, 1972, Headon Hill 7, Bembridge marls (Ile de Wight, Angleterre), MP19-MP20 ; Ehrenstein 1A, Ehrenstein 2, Herrlingen 3 et 6 Weissenburg 8, Möhren 6 (Jura Souabe, Allemagne), MP18-MP19 ; San-Cugat-de-Gavadons, Rocafort-de-Queralt (Bassin de 1'Ebre, Espagne), MP19 ; Ectropomys monacensis Vianey-Liaud et al., 1994, Monac (Gard, France), MP19/20 ; Ectropomys gliriformis (Bruijn et al., 1979), Biniamar (Majorca, Espagne), ?MP19/20.

Caractères dentaires. (Figs. 5, 12, 14 et 15) Dents peu à moyennement semi-hypsodontes et lophodontes ; prémolaires définitives et déciduales relativement petites.

Dents supérieures. L'endolophe est présent, plus bas que le protocône et le métacône, le sinus étant généralement ouvert lingualement; la mure est absente ; le protolophe, long, ferme buccalement le synclinal antérieur (SI) ; le métalophe et le postérolophe sont courts, le premier est transverse, connecté ou non à l'hypocône ou bien orienté distalement et connecté au postérolophe, réduisant ainsi la taille du SIV, qui est alors vestigial.

Dents inférieures. Antérolophide court à moyen, sa partie linguale pouvant être fusionnée ou distincte du métalophide ; l'antésinuside est présent ; le métalophide, transverse, est complet ; le mésolophide peut atteindre l'extrémité de la postmétacristide ou non ; celle-ci ferme lingualement le sII ; l'ectolophide est court et droit, quelquefois interrompu en son milieu sur les dents peu usées ; l'entolophide est complet ; le postérolophide est long, fermant souvent lingualement le synclinide postérieur sIV.

Histoire paléogéographique. D'abord connus en Angleterre méridionale et dans le Jura Souabe (MP18-19), les Oltinomyinae sont ensuite documentés à Majorque (Espagne) et en Quercy (France) (MP19-20). Ils disparaissent au moment de la Grande Coupure. La durée de la sous-famille est de 2,5 Ma.

\section{Sous-famille Patriotheridomyinae nov.}

Genre-type. Patriotheridomys Vianey-Liaud, 1974.

Espèce-type. Patriotheridomys altus Vianey-Liaud, 1974, Escamps (Lot, Quercy, France), MP19.

Diagnose. Palais large et court (bordure postérieure des foramens palatins antérieurs variant du niveau du milieu de P4 à la limite P4-M1); incisives plates, rangée dentaire supérieure courbe ; mandibule avec apophyse coronoïde très basse, ramus horizontal peu élevé et allongé, fosse ptérygoïde profonde.

Dents semi-hypsodontes, à couronne haute. Fort étirement oblique (mésio-buccal à disto-lingual) du protocône. Molaires avec sinus et synclinal II confluents jusqu'à des stades d'usure avancés ; SIV réduit à absent ; sinuside et synclinide III confluents seulement sur les dents peu usées. Chez $P$. altus, dents déciduales très allongées : $\mathrm{dp} 4 \mathrm{~s}$ avec crête préprotoconide et crestules accessoires dans le synclinide antérieur; DP4s avec crestule accessoire délimitant un S0 dans le SI et cuspide prémétacône additionnelle. Antéroconide parfois fort sur p4s avec développement variable d'un synclinide I, plus fréquent mais pas constant - sur les molaires.

\section{Genres et espèces inclus, localités et niveaux} biochronologiques.

Bernardia marandati Vianey-Liaud, 1991, Lavergne (Lot, Quercy, France), MP16;

Patriotheridomys sudrei Vianey-Liaud \& Ringeade,
1993, Gousnat (Tarn-et-Garonne, Quercy, France), MP18a ; Patriotheridomys altus neboulensis Vianey-Liaud, 1978, Sainte Néboule (Lot, Quercy, France), Civrac (Aquitaine, France), Lascours ( Gard, France), MP18b ; Patriotheridomys altus Vianey-Liaud, Escamps (Lot, Quercy, France), Baby II et Ste-Croix-de-Beaumont (Aquitaine, France), MP19.

Caractères dentaires communs à la sous-famille. (Figs. 5, 12,14 et 15) Theridomyidae semi-hypsodontes, à couronne dentaire haute ; fort étirement oblique (mésio-buccal vers disto-lingual) du protocône; molaires (et dents déciduales chez Patriotheridomys) avec sinus et synclinal II confluents jusqu'à des stades d'usure avancés ; SIV réduit à absent ; sinuside et synclinide III confluents seulement sur les dents peu usées.

Remarques. Bernardia est genre frère des Patriotheridomys sur l'analyse partielle AE, l'ensemble étant étroitement apparenté aux Estellomys. Sur l'analyse totale (AG), Bernardia occupe une position phylogénétique au sein des espèces de Theridomys de l'Eocène terminal et du Rupélien. Compte tenu de l'absence de tout élément crânien, des dents déciduales et des prémolaires, très informatives chez les formes hypsodontes, nous considérons comme plus plausible le rapprochement indiqué par l'AE.

Bernardia nov. sp. est signalé par Freudenthal à Olalla 4 (Oligocène basal ; Freudenthal, 1997) qu'il avait auparavant attribué à un Issiodoromyinae indet. Comme aucune figuration ni comparaison avec B. marandati n'est faite, la présence de ce genre dans cette localité est considérée comme douteuse. Ce signalement est seulement accompagné d'une mise en question des relations de parenté entre Elfomys (medius) et Issiodoromys, sans autre argument.

Histoire paléogéographique. Bernardia marandati est connue avec certitude seulement dans une localité du Bartonien (MP16) du sud de la France (Lavergne, Quercy). Patriotheridomys est documenté dans la même région, du début à la fin du Priabonien (MP18a à MP20). Patriotheridomys altus est aussi connu aux Alleveys (Mormont, Jura Suisse ; MP indéterminé, d'après Comte et al., 2012). L'extension temporelle de la sous-famille est d'environ $5 \mathrm{Ma}$.

\section{Sous-famille INDET. 2}

\section{Genre Estellomys Hartenberger, 1971a}

Espèces incluses, localités et niveaux biochronologiques. Estellomys sp. (Comte et al., 2012), Chéry-Chartreuve (Aisne, France), MP15 ; Estellomys ibericus (Thaler, 1966), Sossis, Roc-de-Santa (Bassin de l'Ebre, Espagne), MP17 ; Les Alleveys (Suisse), MP15/16 ; Estellomys cansouni Hartenberger, 1971, Fons 4 (Gard, France), MP17 ; Estellomys varleti (Hartenberger \& Louis, 1976), Grisolles (Aisne, France), MP16.

Caractères dentaires. (Figs. 5 et 14) Dents moyennement semi-hypsodontes ; granules et crestules sur l'émail des bassins ; prémolaires déciduales et définitives de longueur à peine inférieure à égale à celle des molaires (chez Estellomys varleti).

Dents supérieures. Protocône plus lingual que le métacône ; antérostyle présent ; protolophe lié au sommet du protocône ; mésolophe le plus souvent long et bas mais avec des interruptions ; métalophule I vestigial ou absent ; endolophe très réduit à absent et mure ébauchée ou présente ; paraconule réduit et métaconule absent ; métalophule II orienté bucco- 
lingualement, parfois relié à la jonction postérolophe posthypocrista par une courte crête.

Dents inférieures. Antérolophide court, réduit à l'antéroconide (buccal) ou moyennement allongé ; antésinuside et cingulide mésio-buccal ; antérolophulide variablement présent ; métalophide orienté bucco-lingualement, sa partie linguale faisant un angle avec sa partie buccale, les deux pouvant être séparées ; de ce niveau peuvent partir une ou deux crestules mésio-distales plus ou moins longues ; la partie buccale du métalophide s'insère sur le sommet du protoconide ; mésolophide rarement long, plus souvent réduit et toujours bas ; mésostylide petit, souvent relié à la postmétacristide ; mésoconide absent ; ectolophide droit et très court, affaibli ou interrompu au niveau de la jonction avec la préhypocristide.

Remarques. La difficulté à attribuer l'espèce varleti à un genre de Theridomyidae éocène (Vianey-Liaud \& Ringeade, 1993 ; Comte et al., 2012) peut être surmontée ici. En effet, dans nos deux analyses, AG et AE, l'espèce varleti - auparavant attribuée à Blainvillimys - apparaît comme très proche d'Estellomys cansouni. De ce fait, nous avons revu le matériel type des deux espèces. L'attribution de l'espèce varleti au genre Estellomys s'est imposée. Il reste à documenter dans les faunes de rongeurs bartoniennes les loci dentaires manquants pour Estellomys cansouni notamment, et identifier de possibles représentants de ce genre.

Histoire paléogéographique. L'aire de répartition d'Estellomys va du nord de la France (MP15 et MP16) à la Suisse (MP15/16) et s'étend ensuite au sud de la France (MP16) pour finir au nord de l'Espagne (MP17). Ce genre, encore mal identifié dans les localités éocènes, a une extension temporelle d'environ 2 Ma.

\section{Sous-famille Columbomyinae Thaler, 1966}

Genre-type. Columbomys Thaler, 1966, La Colombière (Hérault, France) MP30.

Genres et espèces inclus, localités et niveaux biochronologiques.

Sciuromys cayluxi Schlosser, 1884, A.C. Quercy, Aubrelong 1, Mas-de-Got, La Plante 2, Valbro (Lot, Quercy, France), Lovagny (Haute-Savoie, France), MP21-22 ; Olalla 4A, Montalbán 1D, 3C-E, 11A-B (Teruel, Espagne), MP23 ; Sciuromys sp., in Lacomba \& Morales, 1987, Carrascosadel-Campo (Cuenca, Espagne), MP24/25 ; Sciuromys rigali Mödden, 1993, Belgarric 1, Rigal-Jouet 1 (Tarn-et-Garonne, Quercy, France), MP25a-b ; Sciuromys quercyi Stehlin \& Schaub, 1951, A.C. Quercy, MP indet. ; Sciuromys nov. sp., Espeyrasse (Quercy), MP26.

Columbomys lavocati Thaler, 1962, La Colombière (Hérault, France), MP30 ; Columbomys cuencae Hugueney et al., 1992, Alcorisa (Teruel, Espagne), MP26 ; Columbomys agustii Hugueney et al., 1992, Alcorisa (Teruel, Espagne), MP26.

Caractères dentaires. (Figs. 5, 12, 14 et 15) Dents déciduales plus courtes et étroites que les molaires ; prémolaires à peine plus courtes ou un peu plus longues que les premières molaires.

Dents supérieures. Paracône et métacône renflés ; mésolophe long et plus fin que les autres lophes ; métalophe court connecté au postérolophe ; synclinal IV réduit.

Dents inférieures. Antéroconide, cingulide mésio-buccal et antésinuside forts sur les molaires et absents sur les prémolaires définitives et déciduales, le bord mésial de ces dents montrant une encoche verticale profonde ; métaconide et entoconide volumineux, même chez les espèces hypsodontes ; mésolophide absent (i.e., sII et sIII indistincts) ; sIV presque aussi long (mésio-distalement) que sII + sIII ; développement d'un court néo-mésolophide buccal chez Columbomys ; entolophide complet; postérolophide long.

L'hypsodontie est moins asymétrique que chez les Theridomyinae ou les Issiodoromyinae. Du cément se developpe dans le fond des flexus et flexides à partir de Sciuromys rigali et chez les deux espèces les plus récentes de Columbomys (agustii et lavocati).

Histoire paléogéographique. La répartition des Sciuromys, qui apparaissent au début de l'Oligocène (MP21-MP25), est limitée à l'Espagne et au sud de la France. Leur présence la plus septentrionale est à Lovagny (MP22, Haute-Savoie). La répartition des Columbomys, documentés au Chattien (de MP26 à MP30), est limitée à une zone plus méridionale, en Espagne (MP26-28) et près de la Méditerranée, au sud de la France (MP30). L'extension temporelle de la sous-famille recouvre tout l'Oligocène, soit $11 \mathrm{Ma}$ environ.

\section{Sous-famille Theridomyinae Alston, 1876.}

Beaucoup d'espèces regroupées dans la sous-famille des Theridomyinae ont changé plusieurs fois d'attribution générique (e.g., Lavocat, 1951 ; Thaler, 1966 ; Vianey-Liaud, 1972a, 1972b, 1976, 1989, 1998 ; Bosma, 1974 ; Mödden, 1993 ; Hooker \& Weidmann, 2000 ; Comte et al., 2012) ou même spécifique. Les incertitudes que traduisent ces changements sont notamment liées aux nombreux cas de parallélismes évolutifs qui les affectent, mais aussi à l'augmentation du matériel par rapport aux études initiales et à l'évolution des méthodes d'analyse (e.g., Vianey-Liaud \& Schmid, 2009). Ainsi, ces changements sont aussi dus à la prise en compte de la variabilité morphologique individuelle et la variabilité ontogénétique, cette dernière étant particulièrement forte chez les formes hypsodontes, nombreuses au sein de la sous-famille. Dans cette optique, des changements d'attribution générique ou spécifique (avec des synonymies) sont proposés dans notre étude. Par ailleurs, nous avons inclus dans la liste des espèces attribuées et de leurs localités certains taxons indiqués dans les listes de Freudenthal (1997) et Antunes et al. (1997). Or, la plupart ne sont ni décrits, ni figurés, ni mesurés et comparés avec les populations-types. A la lumière des nouvelles collections et méthodes d'analyse, une révision de certains aspects des faunes espagnoles, notamment de théridomyoïdes, serait nécessaire.

Définition des caractères dentaires de la sous-famille. cf. Vianey-Liaud (1998 : 255); cf. nœud S, p. 21.

Genre-type. Theridomys Jourdan, 1837

Genres inclus. Theridomys Jourdan, 1837 ; Thalerimys Tobien, 1972 ; Blainvillimys Stehlin \& Schaub, 1951 ; Tæniodus Pomel, 1853 ; Protechimys Schlosser, 1884 ; Archaeomys de Laizer \& de Parieu, 1839.

\section{Genre Theridomys Jourdan, 1837}

Espèce-type. Theridomys lembronicus Bravard, 1844.

Espèces incluses, localités et niveaux biochronologiques. Theridomys brachydens Gad, 1987, Möhren-13-19 (Jura Souabe), MP21-22 ; Monac, Ste-Croix-de-Brignon (Gard, France), MP19-20 ; Theridomys calafensis Anadon et al., 1987, 
Calaf, Los Barros, Villarosano 14 (Bassin de l'Ebre, Espagne), Aguatón, Ermita del Puch (Bassin de Teruel, Espagne), MP21; Theridomys golpei Hartenberger, 1973b, San-Cugat-deGavadons (Bassin de l'Ebre, Espagne), Lascours et Ste-Croixde-Brignon (Gard, France), ? La Débruge (Vaucluse, France), Ste-Croix-de-Beaumont (Aquitaine, France), MP18-19; Theridomys bonduelli (Lartet, 1869), Romainville (Marnes de Pantin, Bassin-de-Paris), Ham 1, Lower Hamstead beds, Ile de Wight (Angleterre ; Hooker, 2010), MP19/20 ; Theridomys aquatilis Aymard, 1849, Ronzon (Auvergne, France), Mazan (Vaucluse, France), ?Sanpedor (Bassin de l'Ebre, Espagne), ?Rossemaison (Jura, cf. Becker, 2009), MP21 ; Theridomys nov. sp., Ruch et Pouquette (Aquitaine, France), MP22; Theridomys sp. (= T. lembronicus in Hugueney, 1986), La-Sauvetat (A.C., Auvergne, France), MP23 ; Theridomys major Depéret, 1906, Tárrega, Ciutadilla, Peraltilla, Talladel et Montalbán 1-3-9-11-12-13, Valdecoralles (Bassins de l'Ebre, de Teruel, de Loranca, Espagne), MP23 ; Theridomys lembronicus Bravard in Gervais, 1848, St-Germain-Lembron, Perrier et Cournon (A.C., Auvergne, France), Aubenas-lesAlpes (Provence, France), St-Vincent-de-Barbeyrargues, LesMatelles (Hérault, France), MP25 ; Theridomys octogesensis Arbiol et al., 1997, Mina del Pilar 3, Alcorisa, Bagarella, Ermita del Puch (Bassin de l'Ebre, Espagne), MP26-MP27.

Lorsque la présence de certains taxons n'est attestée que par des listes non argumentées, par des dessins sommaires ou par un petit nombre de spécimens, la validité de certains taxons peut être sujette à discussion ; une incertitude renforcée du fait des parallélismes ou de la variabilité individuelle et ontogénétique. De ce fait, ils ne sont pas tous pris en compte pour l'instant dans la liste ci-dessus et dans les discussions. Il s'agit notamment de Theridomys sp. de Palomera A, Theridomys aquatilis de Palomera B, Theridomys golpei de Route d'Aguatón (Adrover et al., 1983) et Theridomys euzetensis de Deza 2 (PeláezCampomanes, 1996).

Theridomys aff. golpei de Zambrana (Astibia et al., 2000) est en fait Oltinomys platyceps. En effet, la p4 est nettement plus petite que les m1-2s, ce qui n'est pas le cas chez Theridomys golpei, mais l'est chez Oltinomys platyceps. La surface (Lxl) des dents ainsi que leur morphologie sont tout à fait compatibles avec celles d'Oltinomys platyceps : la M1-2 n'a pas de mure et le protolophe rejoint l'apex du protocône, les sinus et SII communiquent profondément ; les deux m1-2s ont un fort antéroconide, prolongé lingualement par l'antérolophide qui ne rejoint pas le sommet du métaconide ; l'antérolophulide est présent et l'antésinuside bien marqué ; l'ectolophide est court et montre une légère interruption sur la dent la moins usée. Se basant sur Crusafont et al. (1971), Cuenca et al. (1992) ont recensé $T$. aquatilis à Calaf 1 ; ils y ont ajouté $T$. calafensis en se référant à Anadon et al. (1987). En fait, ces derniers ont montré que l'espèce aquatilis n'y était pas présente. A Olalla 10, une seule m1-2 est attribuée à T. major (Freudenthal, 1997) sur la seule base de la présence d'un sI, sans figuration, ce qui est discutable compte tenu de la variabilité de ce caractère pris isolément. La question de l'attribution spécifique des quelques dents de Theridomyidae de Santpedor (Bassin de l'Ebre) sera discutée plus loin.

Caractères dentaires et mises au point taxonomiques. (Figs. 6, 12, 16, 17 et 18) La distinction entre les genres Theridomys et Blainvillimys a longtemps porté sur la présence/ absence de synclinide I aux dents inférieures (e.g., Thaler, 1969). Les premières espèces oligocènes de ces deux genres, Theridomys aquatilis et «Blainvillimys » langei, co-existent dans plusieurs localités du début du Rupélien (e.g., Ronzon, en
Haute-Loire ou Mazan, dans le Vaucluse), la première étant de plus grande taille que la seconde. Pour les deux, la fréquence du sI est très faible, elles sont faiblement semi-hypsodontes et leurs sinus et sinusides pénètrent peu dans la largeur de la couronne. Dans le gisement classique de Montalbán (MLB1D, Teruel, Espagne), plus récent, Theridomys major et Blainvillimys « varians » (= helmeri) montrent une fréquence plus grande du sI, plus importante chez T. major que chez $B$. "varians », tandis que la différence de la taille globale s'accroît ; la semi-hypsodontie est plus forte, l'enfoncement des sinus et sinusides plus marqué. L'espèce $B$. gregarius, anciennement décrite par Schlosser (1884) du Quercy (A.C.) a été retrouvée dans diverses localités du Quercy bien repérées et dans un niveau à Montalbán (MLB3C), stratigraphiquement au-dessous de MLB1D. Ses caractéristiques morphologiques sont apparues « intermédiaires » entre celles de «B. » langei et de $B$. helmeri (= varians). Enfin, les espèces Blainvillimys (B. blainvillei) et Theridomys (T. lembronicus) du début du Chattien (e.g., des localités : Aubenas-les-Alpes dans les Alpesde-Haute-Provence, ou bien St-Vincent-de-Barbeyrargues dans l'Hérault), ont des couronnes encore plus hautes. Elles divergent quant à la profondeur du sI, omniprésent et profond chez T. lembronicus, tandis qu'il est très superficiel et disparaît rapidement avec l'usure chez B. blainvillei. On constate ainsi que les effets de l'usure sont d'autant plus marqués que les couronnes sont élevées. Avec l'augmentation de l'hypsodontie, les sinus et sinusides sont encore plus profondément enfoncés horizontalement. Comme par ailleurs le fût des dents demeure relativement vertical chez Theridomys (tandis qu'il est fortement incliné mésio-distalement pour les dents inférieures et linguo-buccalement pour les supérieures chez Blainvillimys) la morphologie des flexus et flexides est plus distincte qu'elle ne l'était pour les espèces du début du Rupélien. Cette inclinaison va aussi de pair avec une plus forte différenciation de l'épaisseur de l'émail chez $B$. blainvillei que chez «B. »langei par exemple. Ainsi, sur la base de ces observations, qui montrent des transformations morphologiques et dimensionnelles progressives, cumulatives au cours du temps et indiquant une même polarité pour des genres différents, on a pu déceler des tendances évolutives parallèles et argumenter le concept de lignées évolutives (Simpson, 1951 ; Thaler, 1966, 1969). Sur cette base notamment, deux grandes lignées ont été proposées (Vianey-Liaud, 1972a), qui présentent un fort parallélisme dans l'accroissement de l'hypsodontie et de la fréquence du sI, comme de l'allongement bucco-lingual des sinus et sinusides. Il s'agissait de la lignée Theridomys aquatilis - T. bonduelli - T. major - T. lembronicus et de la lignée des Blainvillimys («B. » langei - B. gregarius - B. helmeri (= varians ?) - B. blainvillei $-B$. stehlini). Ces deux lignées étaient alors intégrées au genre Theridomys, comme deux sous-genres, Theridomys et Blainvillimys, ce qui traduisait au niveau systématique une certaine parenté morphologique (Vianey-Liaud, 1972a et b). Par la suite, la lignée Blainvillimys sera considérée comme un des trois sous-genres (lignées-sœurs) d'Archaeomys (Protechimys n'en étant pas distingué à ce moment là), avec Toniodus et Archaeomys. Ce choix traduisait alors une proximité morphologique plus forte qu'avec Theridomys et une meilleure adéquation avec les règles taxonomiques (VianeyLiaud, 1979). Finalement, chacun de ces taxons retrouvera un statut générique propre (Vianey-Liaud, 1989).

Pendant longtemps, la lignée des Theridomys ne connaîtra pas de modifications majeures, à l'exception d'une certaine ambiguïté sur la position de T. bonduelli. Cette dernière 
espèce est très proche morphologiquement de T. aquatilis, mais le sI y est beaucoup plus fréquent et plus grand. Cette morphologie de T. bonduelli, " intermédiaire » entre celle de $T$. aquatilis et de T. major, justifiait alors le placement de $T$. bonduelli dans la lignée. Mais d'emblée, la discordance entre la position biochronologique inférée par l'hypothèse de la lignée, les informations fournies par le niveau stratigraphique d'où provenait T. bonduelli (Marnes-de-Pantin) et par les rares autres mammifères associés, a posé question (Vianey-Liaud, 1972b : 359). Par la suite, l'espèce a été considérée comme représentant l'un des membres d'une autre lignée (VianeyLiaud, 1979).
A la présence du sI, d'occasionnelle à quasi constante chez l'espèce-type ( $T$. lembronicus) et chez $T$. major, s'est ensuite ajouté un autre critère différenciant Theridomys et Blainvillimys : la régularité de l'épaisseur de l'émail sur les flancs opposés des flexus et flexides (Vianey-Liaud, 1998). En effet, entre temps, d'autres espèces avaient été décrites et attribuées au genre Theridomys, «T. » perrealensis (VianeyLiaud, 1977), T. ludensis (Vianey-Liaud, 1985), T. brachydens (Gad, 1987), T. calafensis (Anadon et al., 1987) et T. margaritae (Vianey-Liaud, 1989). Le sI est ébauché («T. » perrealensis), très peu fréquent chez $T$. margaritae, comme c'est le cas chez T. aquatilis et il est absent chez les autres. Ce critère de l'émail
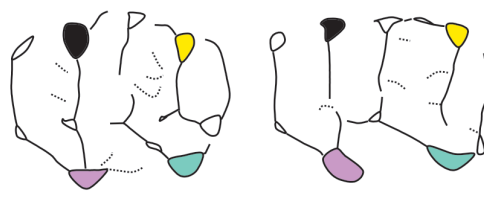

Estellomys sp. (Chéry-Chartreuve)

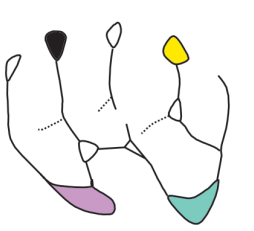

Paratheridomys margaritae (Hoogbutsel)

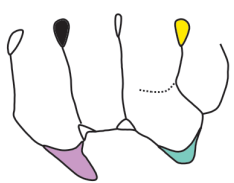

"BI." gousnatensis

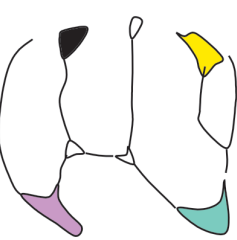

Blainvillimys gemellus (La-Plante 2)

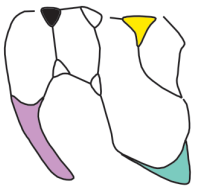

Tœniodus curvistriatus

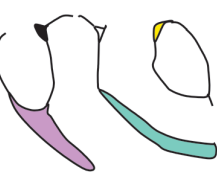

Protechimys gracilis
Estellomys varleti
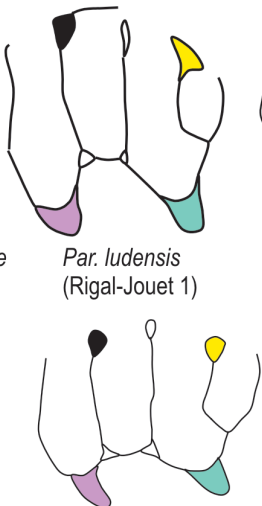

"BI." pseudosiderolithicus

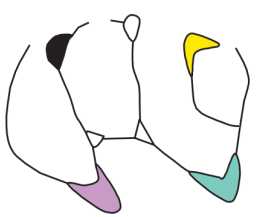

Blainvillimys gregarius (Mas-de-Got)

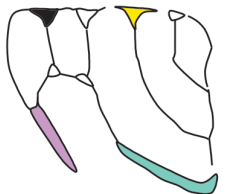

Tœniodus hexalophodus

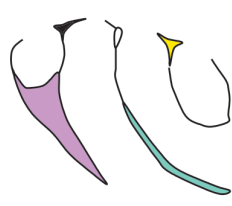

Prot blainville

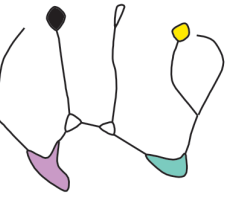

Theridomys golpei

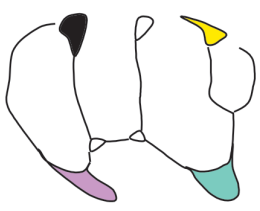

Theridomys calafensis

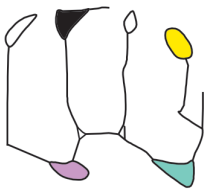

"Bl." civracensis

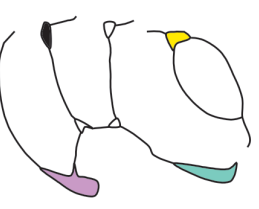

Blainvillimys helmer (Pech-Crabit)

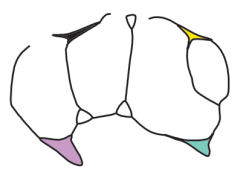

Protechimys truci

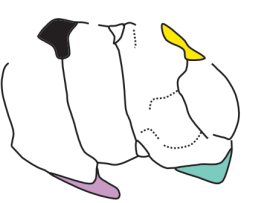

"Theridomys" bonduelli

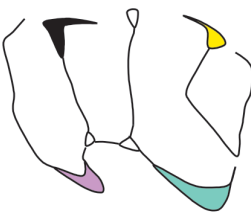

Theridomys major (Montalban)

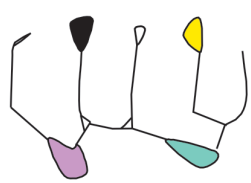

"BI." rotundidens

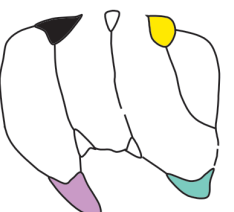

Bl. heimersheimensis (Heimersheim)

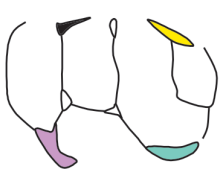

Prot. lebratierensis

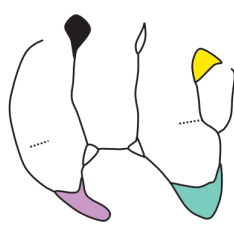

"Theridomys" aquatilis

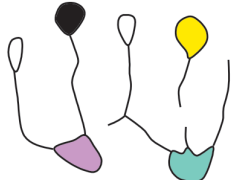

"Blainvillimys" euzetensis (Type)

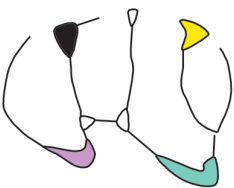

"Blainvillimys" lange (Aubrelong1)

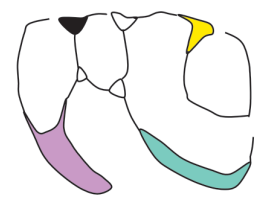

Blainvillimys blainvillei

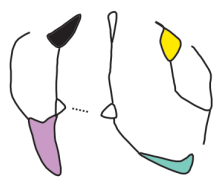

Prot. variabilis

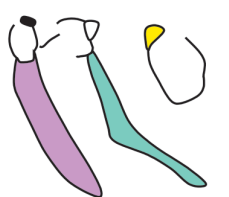

Prot. major (Les-Milles)

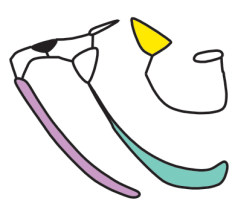

Archaeomys / Blainvillimys robustus (St-Henri)

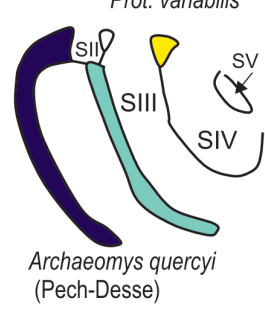

Paracône $\square$ Métacône $\square$ Protocône $\square$ Hypocône $\square$ Autres cuspides \Trajets des lophes et crestules Variations des lophes et crestules

Figure 16. Schémas des M1-2s supérieures d'Estellomys et de Theridomyinae. 
s'était imposé comme facteur discriminant et permettait de répartir les espèces de l'Eocène supérieur entre Theridomys et Blainvillimys (Vianey-liaud \& Ringeade, 1993). En effet, certaines d'entre elles étaient référées « pragmatiquement » (Bosma, 1974) au genre «fourre-tout » Isoptychus, pour regrouper tous les théridomyinés peu hypsodontes. Or, l'espècetype de ce genre est l'espèce aquatilis, de l'Oligocène inférieur. Déjà, Stehlin \& Schaub (1951 : 37) faisaient remarquer que Jourdan (1837) n'avait pas nommé d'espèce lorsqu'il avait créé le genre Theridomys pour des rongeurs notamment de Ronzon et de Perrier. De ce fait, ils ont considéré qu'aquatilis étant le rongeur le plus abondant à Ronzon, et lembronicus à Perrier, les deux espèces pouvaient être celles ayant servi à la définition originelle de ce genre. Par la suite Gervais (1852) et Pomel (1853) ont penché pour lembronicus comme espèce-type du genre et Pomel a créé Isoptychus pour aquatilis. Stehlin \& Schaub ont repris la proposition de Croizet (in Bravard, 1844) à savoir Perrieromys pour lembronicus, proposition qui n'a pas été retenue par la suite. Ils ont critiqué la définition d'Isoptychus, parce que les synclinaux externes n'étaient pas tous de même taille. Le genre Isoptychus fut encore utilisé par Hooker \& Weidmann (2000) car la monophylie du genre Theridomys n'était pas clairement établie.

Il est clair à présent - et ce depuis longtemps - que le genre Theridomys inclut plusieurs lignées spécifiques indépendantes et que démêler leurs relations de parenté semblait être un prérequis nécessaire afin d'attribuer des noms de genres différents pour chacune de ces lignées. Si beaucoup des espèces éocènes ne répondent pas à la définition de Theridomystype, toutes ne correspondent pas plus à celle d'Isoptychus. Rapporter ces espèces, éocènes ou de l'Oligocène inférieur, au genre Theridomys sur la base de l'épaisseur de l'émail n'était sûrement pas suffisant, mais dans tous les cas, cela ne correspondait pas à une séparation en grades d'hypsodontie différente. L'accroissement en hauteur des couronnes est une tendance générale chez beaucoup de Theridomyidae. Notre choix d'alors (e.g., Schmidt-Kittler \& Vianey-Liaud, 1987 ; Vianey-Liaud et al., 1995) reflétait l'état encore imparfait de l'étude de ces espèces, mais il traduisait aussi notre expérience en matière d'analyse des effets de l'évolution du taux d'hypsodontie sur la variabilité de la morphologie dentaire.

Dans notre analyse, les nœuds soutenant les différentes branches de Theridomys sont peu robustes, hormis celui qui soutient les trois espèces terminales de Theridomys ( $T$. major, $T$. lembronicus et $T$. octogesensis) et celui qui soutient le clade «T. » margaritae et «T. » ludensis (Fig. 7B). Les relations entre les autres espèces ne sont pas claires pour l'instant. Si une étroite parenté entre « $T$. » ludensis et « $T$. » margaritae avait été supposée, l'appartenance au même genre de l'espèce vassoni était plus problématique (Vianey-Liaud, 1989 : 212-214) mais il semblait encore prématuré de formaliser cela au plan taxonomique en créant alors un nouveau genre. Dans notre analyse globale, l'espèce vassoni avait été écartée en raison du faible nombre de loci disponibles. Souhaitant tester sa relation avec les autres Theridomys, nous l'avons introduite dans une nouvelle analyse globale, réalisée de la même manière qu'AG. L'espèce vassoni se positionne alors en branchement intermédiaire entre margaritae et ludensis. Considérant les espèces de Theridomys des localités du Jura Souabe, Gad (1987) postulait une relation entre «T. $»$ brachydens et «T.» ludensis, ce qui n'est pas soutenu par les résultats de l'analyse AG. "Theridomys » brachydens se positionne auprès des Thalerimys, mais de façon très peu robuste. Sur AO, elle est la branche basale de tous les Theridomys. Pour l'instant, nous la considèrerons dans le genre «Theridomys ». Par ailleurs, des populations ont été attribuées à « $T$. » brachydens dans des niveaux plus anciens (MP19-20 ; Vianey-Liaud \& Ringeade, 1993), qu'il conviendrait de réviser et notamment comparer au matériel type de l'espèce.

Les espèces du clade de margaritae - ludensis sont attribuées à un nouveau genre : Paratheridomys nov. gen., dont l'espècetype est $P$. ludensis. L'espèce vassoni est rapportée avec doute à ce nouveau genre (cf. p. 41 ci-dessous). Les espèces de Paratheridomys se différencient très clairement des espèces du clade des Theridomys oligocènes qui comprend Theridomys lembronicus (MP25), espèce-type du genre Theridomys.

Trois espèces au moins sont actuellement connues dans l'Eocène terminal : " Theridomys » cf. brachydens, " Theridomys » golpei et "Theridomys » bonduelli. «Theridomys » golpei est documenté à la fois dans le bassin de l'Ebre et aussi dans le sud de la France (MP18-MP19/20). Dans l'Oligocène inférieur, on connaît « $T$. » brachydens, « $T$. » calafensis, «T. » aquatilis (MP21), T. nov. sp. (MP22) et T. major (MP23), et dans l'Oligocène supérieur, T. lembronicus (MP25) et T. octogesensis (MP26). Dans le matériel déterminé comme T. lembronicus de La-Sauvetat (A.C., Auvergne ; collections du Natural History Museum (NHMUK) de Londres et du Muséum National d'Histoire Naturelle [MNHN] à Paris), Hugueney (1986) note que cette espèce est plus petite que celle d'Antoingt. La vingtaine de spécimens mentionnés n'est pas figurée. Un spécimen du NHMUK indiqué comme provenant d'Antoingt est attribué à T. lembronicus (ANT1; Vianey-Liaud, 1972b, fig. 12-1). Cependant, ce spécimen se différencie par une taille plus petite que les spécimens de St-Germain-Lembron et Perrier (A.C., Auvergne) et présente aussi un sinuside plus court et le sIII plus long. Cette suite de caractères exprimerait plutôt un stade évolutif moins avancé. Nous discuterons ultérieurement certains aspects de "l'assemblage " des Theridomyidae de La-Sauvetat (p. 60). Pour le moment, considérons seulement les sites stratifiés renfermant $T$. lembronicus, exploités au cours du $20^{\text {ème }}$ siècle et qui sont les seuls à même de permettre le contrôle des caractères propres à un seul taxon au sein du genre. Il s'agit des sites de St-Vincent-de-Barbeyrargues, LesMatelles (Hérault) et Aubenas-les-Alpes (Alpes-de-HauteProvence), qui renferment par ailleurs d'autres rongeurs théridomyoïdes aux caractères différents (Blainvillimys blainvillei, Protechimys blainvillei et/ou Protechimys gracilis). Une espèce cf. lembronicus, plus petite que le type, est signalée à Terrenoire (Hugueney, 1994). Sa taille, la morphologie des DP4s et dp4s notamment, tout comme celle des molaires, si ce n'est un enfoncement de l'antésinus et du sinuside moins marqués, rappellent plutôt Blainvillimys blainvillei, notamment celui d'Aubenas-les-Alpes.

Les rapports entre Theridomys bonduelli et les espèces chez lesquelles le sI est présent sur la grande majorité des dents inférieures (comme T. major - T. lembronicus - T. octogesensis) ne sont pas définitivement établis. Y-a-il un lien de parenté entre bonduelli, fini-éocène et les Theridomys oligocènes (de MP23 à MP26) ? Nos résultats phylogénétiques et notre analyse de ces derniers, ne nous permet pas de choisir entre un maintien de bonduelli au sein de Theridomys ou un retour dans le genre Trechomys que Lartet (1869) avait créé pour l'espèce.

" Theridomys » golpei (Eocène supérieur) est de taille supérieure à celle de «T. » aquatilis (Oligocène basal), de morphologie moins spécialisée, avec une dp4 moins allongée antérieurement ; la dp4 de «T. » golpei est beaucoup moins 
longue que celle de «T. » bonduelli. La DP4 d'aquatilis type de Ronzon n'est pas connue ; celle de «T. . golpei est relativement courte et pourvue de petites crestules accessoires, si on la compare avec celle de $T$. calafensis (MP22) plus longue et avec une subdivision du synclinal antérieur.

A Ruch et Pouquette (Aquitaine), une espèce de Theridomys (Theridomys sp.), encore inédite, montre des caractéristiques morphologiques intermédiaires entre celles de «T.» aquatilis ou $T$. calafensis et $T$. major. Ses dents déciduales sont plus allongées antérieurement que celles de «T. » aquatilis ou $T$. calafensis (Figs. 6 et 12). Le lobe antérieur de ses dp4s montre plusieurs crestules qui subdivisent le synclinide antérieur (sI) alors qu'il n'est pas subdivisé chez «T. » aquatilis de Ronzon (Vianey-Liaud, 1972b, fig. 1-g) et qu'il présente une subdivision incomplète avec une seule crestule accessoire courte chez T. calafensis. Le lobe antérieur des DP4s comporte un synclinal antérieur additionnel (S0), parfois lui-même subdivisé comme chez T. major alors qu'il ne l'est pas chez $T$. calafensis. Theridomys sp. montre aussi des dents un peu plus grandes et plus hypsodontes que chez $T$. aquatilis et nettement moins grandes, moins hypsodontes et moins téniodontes que celles de T. major. Par ailleurs, le synclinide I des molaires inférieures est rare, comme chez $T$. aquatilis et $T$. calafensis, alors qu'il est beaucoup plus fréquent chez T. major.

Ainsi, toutes les espèces de Theridomys du début de l'Oligocène inférieur montrent une fréquence faible du sI aux dents inférieures, contrairement à « $T$. » bonduelli ou à $T$. major-lembronicus. Par ailleurs, des espèces supposées subcontemporaines, $T$. calafensis en Espagne, T. sp. en Aquitaine diffèrent par leur taille, la première étant plus grande. D'autre part, T. calafensis est plus grand et ses dents sont surtout bien plus larges que celles de T. major. Enfin, cette dernière espèce n'est recensée qu'en Espagne. Cependant, il n'est pas impossible qu'elle ait été présente dans le matériel des anciennes localités d'Auvergne. En effet, la pièce identifiée comme T. lembronicus dans les collections du NHMUK (ANT1; Vianey-Liaud, 1972b, fig. 12-1) montre un sIII moins réduit et un sinuside plus long que sur les spécimens typiques de T. lembronicus et elle est un peu plus petite, s'approchant ainsi des caractéristiques de T. major.

Notre analyse ne montre pas de lien phylogénétique étroit entre «T.» aquatilis, T. calafensis et T. major. Il ne faut cependant pas perdre de vue que, sur notre cladogramme (Figs 7B et 9), les noeuds séparant tous ces « Theridomys» du clade bien identifié Theridomys major- lembronicus octogesensis sont très mal soutenus $(2<\mathrm{IB}<5)$. Près de $3 \mathrm{Ma}$ au moins séparent les deux espèces aquatilis et major, et 3 Ma major et lembronicus, ce qui démontre l'étendue des lacunes dans nos connaissances de l'évolution des Theridomys. Par ailleurs, diverses localités du bassin de Teruel (Espagne : Freudenthal, 1997) ont livré T. major, qui s'étendrait ainsi sur deux niveaux biochronologiques, de MP22 à MP23. Il serait intéressant de comparer deux populations bien distinctes dans le temps afin de repérer d'éventuelles différences de degré évolutif. Theridomys octogesensis n'est connue que de deux localités du bassin de l'Ebre; elle est bien définie par sa grande taille, sa forte téniodontie (forte obliquité des lophes), l'enfoncement des sinus et sinuside, l'allongement antérieur des dents inférieures ( $\mathrm{s} 0$ même sur les prémolaires et les molaires) et postérieur des dents supérieures (SV même sur les prémolaires et les molaires).

Histoire paléogéographique. Les plus anciennes espèces attribuées aujourd'hui au genre Theridomys datent du Priabonien supérieur (MP18-19) et sont localisées dans la moitié sud de la
France (Aquitaine, Provence) et au nord de l'Espagne. Elles se retrouvent ensuite sur tout le territoire depuis le nord de l'Espagne jusqu'en Angleterre et au Jura Souabe (MP19-20). Les espèces oligocènes sont documentées du Bassin-de-Paris en Suisse, jusqu'au sud de la France et en Espagne (MP21-25). Leur aire de répartition se restreint ensuite et l'espèce la plus récente est connue seulement au nord de l'Espagne (MP26-27). L'extension temporelle du genre recouvre environ $10 \mathrm{Ma}$.

\section{Genre Paratheridomys nov. gen.}

1sid:zoobank.org:act:EB664D06-F0C2-473C-AC52C11FDDFAD1D5

Espèce-type. Paratheridomys ludensis (Vianey-Liaud, 1985), Rigal-Jouet 1 (Tarn-et-Garonne, Quercy, France), MP25.

Diagnose du genre. Theridomyinae dont la branche horizontale de l'os dentaire est peu élevée, comparée à celle de Theridomys; incisive plus étroite et plus courte que chez Theridomys; épaisseur de l'émail peu différenciée sur les flancs opposés des lophes et lophides. Surface de l'émail lisse ; dents lophodontes mais cuspides principales (paracône, métacône, métaconide et entoconide) renflées ; semi-hypsodontie modérée.

Espèces incluses, localités et positions biochronologiques. Paratheridomys margaritae (Vianey-liaud, 1989), Hoogbutsel (Belgique), MP21, ? Neustadt (Marburg, Allemagne), MP20, La Plante 2 (Lot, Quercy, France), MP22, Bernloch (Jura Souabe), Gas et Maintenon (Bassin-de-Paris), MP23-24 ; ? Paratheridomys vassoni (Pomel, 1853), La Sauvetat (A.C., Auvergne, France), ? MP 22/23 ; Paratheridomys ludensis (Vianey-liaud, 1985), Lébratières 14, Rigal-Jouet 1 et Garouillas (Tarn-et-Garonne et Lot, Quercy, France), Habach 4-5 (Jura Souabe, Allemagne), MP24-25 (=Isoptychus bumbachensis Mayo, 1987, Bumbach, Suisse)

Caractères dentaires. Dents supérieures. Dents déciduales un peu plus longues que les prémolaires définitives, pourvues d'un synclinal additionnel antérieur S0 ; présence occasionnelle de courtes crestules dans les SI et SII du côté buccal ; P4s nettement plus grosse que M1s ; sur les molaires, antérolophe généralement long, mésostyle fort à réduit, SII ouvert buccalement, prémétacrista forte, postérolophe court à très court et non connecté au métacône et synclinal postérieur (SIV) transverse, court à très réduit.

Dents inférieures. Dents déciduales peu allongées, sans s0, à peine plus longues que les prémolaires définitives, protoconide allongé mésio-distalement, faiblement proéminent ; p4s nettement plus allongées que les $\mathrm{m} 1 \mathrm{~s}$; mésolophide raccourci ou s'amincissant lingualement, séparé ou relié à la crête métastylide ; mésostylide réduit ; sI très rare ou absent des molaires ; postprotocristide modérément longue ; ectolophide continu; m3s plus courtes que les molaires.

Histoire paléogéographique. Le genre est connu d'abord dans le Bassin de Mainz en Allemagne (Neustadt, MP20), si la forme décrite comme «Theridomys aquatilis » par Tobien, (1972) peut lui être attribuée. Il est ensuite documenté en Belgique (Hoogbutsel, MP21) puis en France à partir de MP22. Le genre est enfin réparti du Jura Souabe au sud de la France, en passant par le Jura Suisse à la transition Rupélien-Chattien (MP24-25). L'extension temporelle du genre est de plus de $7 \mathrm{Ma}$. 
Genre Thalerimys Tobien, 1972

Espèce-type. Thalerimys fordi (Bosma \& Insole, 1972), Osborne beds (Ile de Wight, Angleterre).

Espèces incluses, localités et niveaux biochronologiques. Thalerimys perrealensis Vianey-liaud, 1977, La-Débruge (Vaucluse, France), MP18 ; Thalerimys headonensis (Bosma, 1974), Headon Hill 1, Whitecliff Bay 1 (Ile de Wight, Angleterre), MP18; Thalerimys fordi (Bosma \& Insole, 1972), Lacey's Farm Quarry, Totland Bay, Headon Hill 2, MP18/19?, Neustadt, Nordhausen, (Marburg, ouest du fossé rhénan, Allemagne), MP20.

Caractères dentaires et remarque taxonomique. L'espèce perrealensis est ici attribuée à ce genre. Les dents supérieures et inférieures des trois espèces portent des crestules accessoires le long des lophes et lophides et elles sont plus fortes chez Th. headonensis.

Denture supérieure. Parmi le matériel inédit de La-Débruge (MP18b), une DP4 a pu être attribuée à « $B$. » pseudosiderolithicus, une autre à Thalerimys perrealensis (Fig. 19). La DP4 de Th. perrealensis est morphologiquement très semblable à celle de Th. headonensis, avec le parastyle et l'antérostyle marqués, une seule crestule accessoire précédant le protolophe dans le SI, le mésostyle dédoublé et le métalophe relié au postéroconule. Les $\mathrm{P} 4 \mathrm{~s}$ de ces trois espèces sont de taille inférieure à presque égale à celle des molaires ; leurs morphologies sont très proches, avec l'antérolophe libre à son extrémité buccale, les crestules accessoires qui peuvent découper le SII en son milieu (Th. perrealensis et Th. fordi). $\mathrm{La}$ différence principale entre les $\mathrm{P} 4 \mathrm{~s}$ de $T h$. perrealensis et celles des autres espèces du genre réside dans le dédoublement

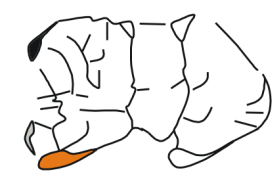

Thalerimys headonensis

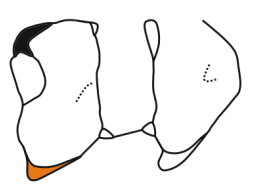

"Theridomys" bonduelli

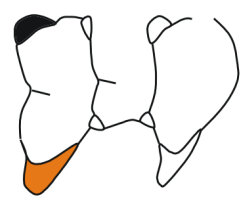

Theridomys calafensis

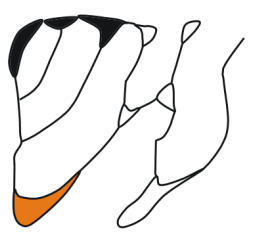

Theridomys octogesensis

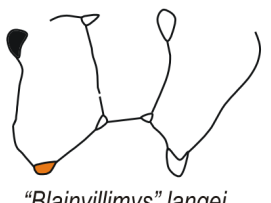

"Blainvillimys" langei

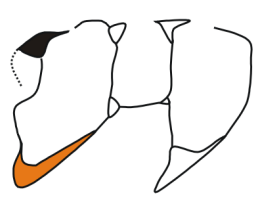

Blainvillimvs blainvillei

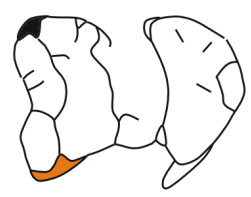

Thalerimys fordi

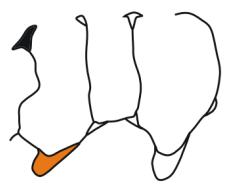

"Theridomys" golpei

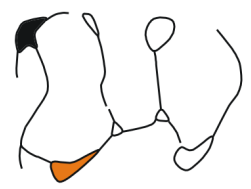

"Thalerimys" perrealensis

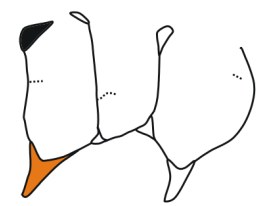

"Theridomys" brachydens (Möhren 13)

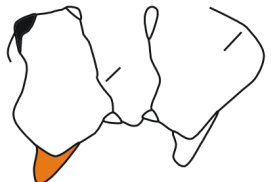

Paratheridomys margaritae

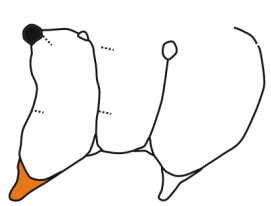

Theridomys sp. (Möhren 13)

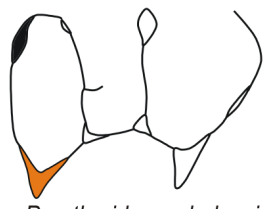

Paratheridomys ludensis

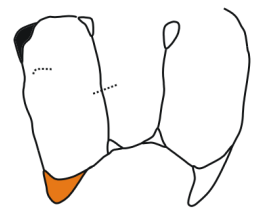

"Theridomys" aquatilis
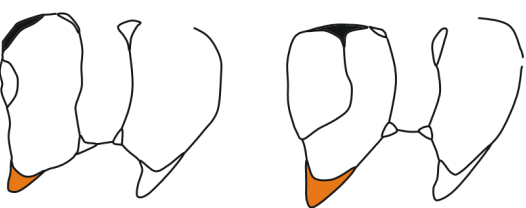

Theridomys major

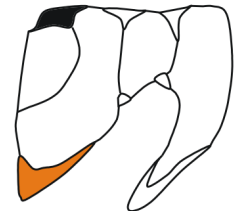

Theridomys lembronicus
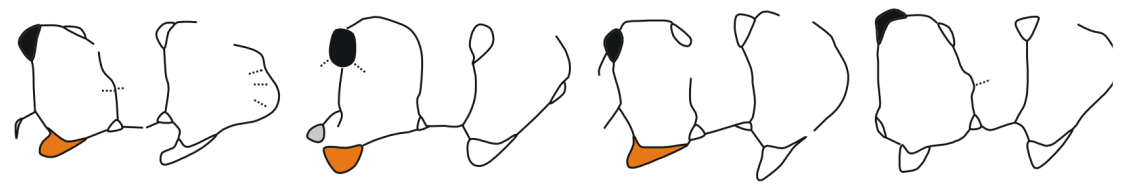

"Blainvillimys" gousnatensis

"B." Pseudosiderolithicus

"B." civracensis

"B." rotundidens

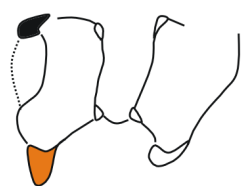

Blainvillimys gregarius

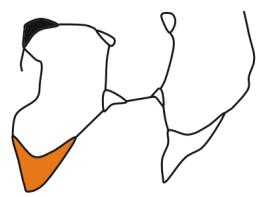

Blainvillimvs campinsensis

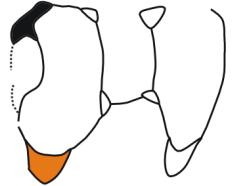

Blainvillimys gemellus

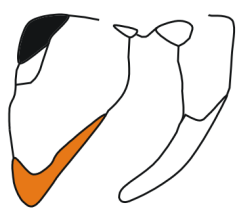

Blainvillimvs stehlini
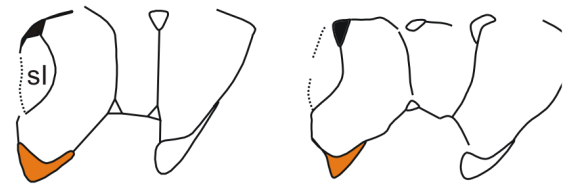

Blainvillimys helmeri Blainvillimys heimersheimensis

Figure 17.Schémas des m1-2s inférieures de diverses espèces de Theridomyinae. 
du mésostyle, voire de la partie buccale du mésolophe, amorcé chez Th. headonensis, plus fréquent chez Th. fordi et absent chez Th. perrealensis. Il faut cependant souligner que la variabilité morphologique de $T h$. perrealensis est mal connue, faute de matériel suffisant.

Denture inférieure. Les p4s montrent, aussi bien chez Th. perrealensis que chez Th. headonensis, une partie antérieure à peine moins large que la partie postérieure, avec l'antérolophide court et bas ; les deux ml-2s connues portent aussi une encoche mésio-linguale courte et l'une d'entre elles un court antésinuside mésio-buccal. Chez Th. headonensis cependant, l'antérolophide est plus clairement mésio-buccal, tandis qu'il est surtout mésio-lingual chez Th. perrealensis. Chez les deux espèces, les $\mathrm{p} 4 \mathrm{~s}$ sont de taille sub-égale aux $\mathrm{m} 1 \mathrm{~s}$; elles sont relativement plus longues chez Th. fordi.

Sur quelques m1-2s de Th. perrealensis et Th. headonensis et sur toutes celles de Th. fordi, un sI se forme par élévation et allongement de l'antérolophide. Ce dernier est parallèle au métalophide, anguleux, lequel joint le sommet du métaconide au sommet du protoconide. De nombreuses crêtes accessoires transverses, parallèles au mésolophide, partitionnent le synclinide antérieur des dp4s de Th. fordi. Elles sont moins nombreuses et plus obliques chez Th. headonensis.

Histoire paléogéographique. Ce genre est bien documenté sur l'Ile de Wight au Priabonien supérieur (MP18-MP19) et il est aussi présent plus au sud, comme en témoigne l'espèce Th. perrealensis de La-Débruge (Vaucluse, France). Il semble avoir évolué rapidement, si l'on considère que les deux espèces Th. headonensis et Th. fordi constituent deux stades évolutifs d'une même lignée. Celle-ci aurait étendu son aire de répartition dans le sud-ouest de l'Allemagne (MP20 ?). Son extension temporelle est d'environ $2 \mathrm{Ma}$.
Genre Blainvillimys Stehlin \& Schaub, 1951

Espèce-type. Blainvillimys blainvillei (Gervais, 1848) ; Antoingt (A.C., Auvergne), LIM 539, fragment de mandibule avec m1-m3, MNHN, Paris.

Espèces incluses, localités et niveaux biochronologiques. « Blainvillimys » euzetensis (Depéret, 1917), Euzet, ? Fons 4, ? Malpérié (Tarn et Garonne, Quercy, France), MP16-17 ; «Blainvillimys » gousnatensis (Vianey-liaud, 1976), Gousnat (Tarn-et-Garonne, Quercy, France), MP18a ; « Blainvillimys » civracensis Vianey-Liaud \& Ringeade, 1993, Civrac (Aquitaine, France), Ste-Néboule (Lot, Quercy, France), MP18b ; "Blainvillimys » pseudosiderolithicus (de Bonis, 1964), La-Débruge (Vaucluse, France), Aguatón 2D (Teruel, Espagne), MP18, Headon-Hill 2-4, (Ile de Wight, Angleterre), MP18-19 ; " Blainvillimys » rotundidens Schlosser, 1884, A.C. Quercy, Escamps, Tabarly (Lot, Quercy, France), Baby II (Aquitaine, France), MP19-20 ; «Blainvillimys » langei Vianey-Liaud, 1972b, Aubrelong 1, Ravet (Lot et Tarn-etgaronne, Quercy, France), Mazan (Vaucluse, France), MP21 ; Blainvillimys gregarius (Schlosser, 1884), Mas-de-Got A-B, Gardiol 3, Coulou, La Plante 2, Cavalé, Valbro, Baraval (Lot, Quercy, France), Ruch, Fumel et Pouquette (Aquitaine, France), Lovagny (Savoie, France), MP22 ; Blainvillimys gemellus Vianey-Liaud, 1989, La Plante 2, Valbro, Baraval (Lot, Quercy, France), La-Sauvetat (A.C., Auvergne, France), MP22; Blainvillimys helmeri Vianey-Liaud, 1972b (= ?Blainvillimys varians Thaler, 1969), Les-Chapelins (Vaucluse, France), Pech-Crabit, Itardies (Lot, Quercy, France), Montalbán (Teruel, Espagne), MP23 ; Blainvillimys blainvillei (Gervais, 1848) Antoingt (A.C., Auvergne, France), Rigal-Jouet 1 (Tarnet-Garonne, Quercy, France), Le-Garouillas et Belgarric 1

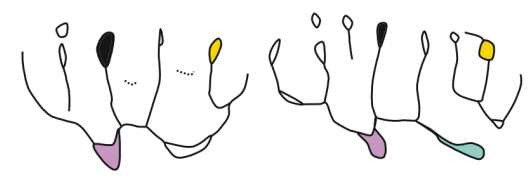

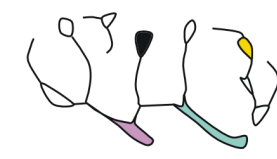

"Blainvillimys" langei

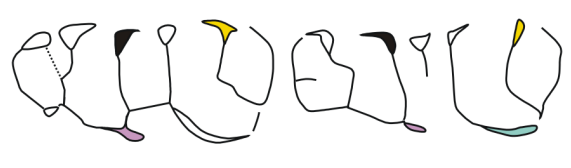

Blainvillimys gregarius Blainvillimys gemellus

"Blainvillimys" pseudosiderolithicus "BI." civracensis

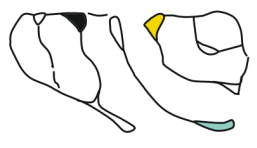

Toeniodus hexalophodus

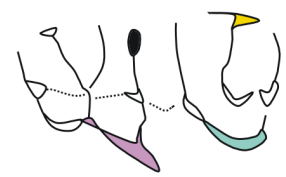

Blainvillimys helmeri

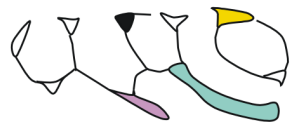

Blainvillimys blainville

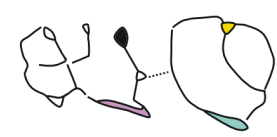

Protechimys lebratierensis

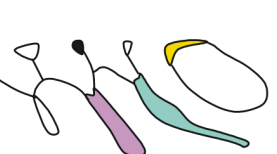

Archaeomys ehrensteini

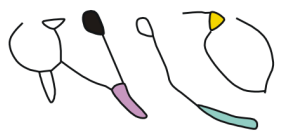

Protechimys gracilis

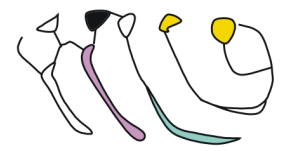

Archaeomys huerzeleri

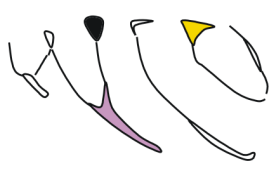

Protechimys blainvillei

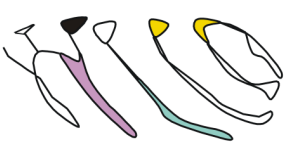

Archaeomys intermedius

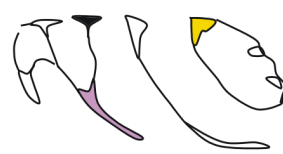

Protechimys major

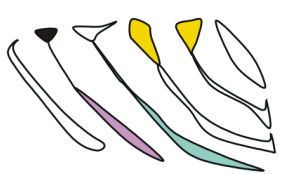

Archaeomys laurillardi

Paracône $\square$ Métacône $\square$ Protocône $\square$ Hypocône $\square$ Autres cuspides 

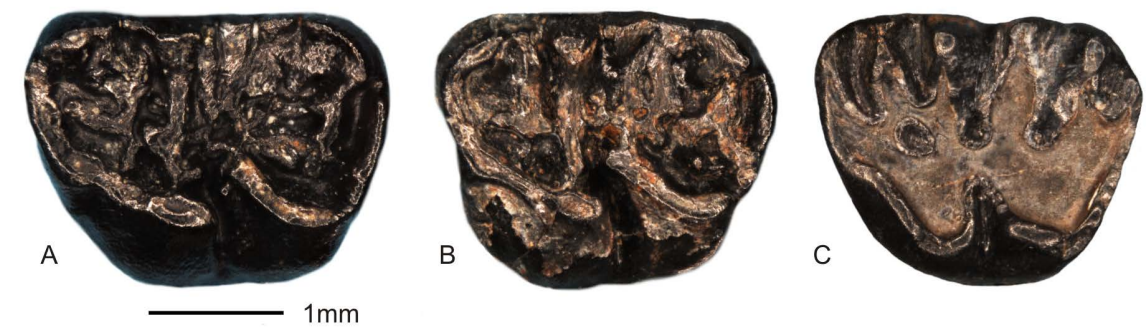

Figure 19. A. UM-HH4-5, DP4 gauche de Thalerimys fordi Bosma \& Insole, des Headons beds (Ile de Wight, sud de l'Angleterre, niveau HH4), MP18-19 ; B. UM-DEB 14, DP4 gauche de Thalerimys perrealensis Vianey-Liaud, de La Débruge (Vaucluse, France), MP18 ; C. UM-DEB 12, DP4 droite (inversée) de « Blainvillimys » pseudosiderolithicus de Bonis 1964, de la Débruge (Vaucluse, France) MP18.

(Lot, Quercy, France), St-Martin-de-Casselvi (Tarn, France), Aubenas-les-Alpes (Alpes-de-Haute-Provence, France), St-Vincent-de-Barbeyrargues, Les-Matelles (Hérault, France), Mümliswyl (Suisse), Campins (Espagne), MP25 [= Blainvillimys campinsensis (Arbiol, 1993) Can Quarante Campins (Espagne)], Blainvillimys sp. 1, (= T. cf. lembronicus in Hugueney, 1994), Terrenoire (Vaucluse, France), MP24/25 ; Blainvillimys sp. 2 (in Vianey-Liaud, 1998), St-Martin-deCastillon, > MP23 < MP24 ; Blainvillimys stehlini Mayo, 1987, Mümliswyl (Suisse), MP 26 ; ? Blainvillimys avus (Stehlin \& Schaub, 1951), A.C. Quercy, Lébratières 14 (Lot, Quercy, France), Vialenc (Cantal, France), Appelle (Haute-Garonne, France), MP24.

Remarques taxonomiques. A la lumière des résultats issus de l'analyse AG (Fig. 7B), les espèces attribuées au genre Blainvillimys constituent deux clades séparés par les Theridomys - Thalerimys au nœud T. Les caractères qui séparent le premier clade «Blainvillimys » se retrouvent par convergence au nœud U pour séparer le deuxième clade Blainvillimys des autres Theridomys. Il s'agit de : l'hypsodontie plus forte, la différence d'épaisseur de l'émail sur les flancs opposés des lophes, lophides, sinus et sinuside, la présence d'un antésinus sur les $\mathrm{DP} 4 \mathrm{~s}$, la longueur des $\mathrm{m} 3 \mathrm{~s}$ plus réduite, le sI des dp4s fermé lingualement, mésialement et buccalement, les paraconide et métaconide n'émergeant pas au-dessus des lophides. En considérant les résultats de l'analyse cladistique, ces caractères sont interprétés comme des homoplasies (hypothèses d'homologies primaires non soutenues). S'y ajoutent les caractères dérivés partagés avec Toniodus et Archaeomys qui contraignent le rapprochement des Blainvillimys oligocènes avec ces deux genres. Il s'agit notamment de l'allongement et de l'obliquité des sinus - sinuside, de la présence chez tous du S0 aux DP4s, du postérolophe relié à l'hypocône et au métalophe, du développement de l'antéroconide aux $\mathrm{p} 4 \mathrm{~s}$, de la présence variable d'un sI très peu profond et souvent réduit à un îlot et de la possibilité - variable - d'une communication sIV - sinuside. Le nœud V1 correspond aux espèces de Blainvillimys de l'Oligocène (excepté « B. » langei). Dans ce clade, les espèces s'organisent en branchements successifs, traduisant certes une parenté entre ces formes, mais sans aucune discrimination des schémas de « lignées parallèles », et notamment des espèces de ces lignées distinctes qui peuvent se retrouver au sein d'une même localité (e.g., Vianey-Liaud, 1989, 1998).

Du fait de la séparation des deux clades de Blainvillimys, il serait tout à fait légitime de proposer un nouveau genre pour les espèces du premier clade, le second contenant l'espècetype du genre Blainvillimys. Cependant « B. » langei (espèce du Rupélien basal) qui est incluse dans le premier clade, est très proche de B. gregarius (second clade), les différences entre les deux espèces étant essentiellement de nature graduelle (e.g., hypsodontie, téniodontie, fréquence du sI). Cette similarité morphologique se traduit aussi dans leur anatomie crânienne et mandibulaire.

Aussi, bien qu'apparaissant comme deux clades distincts et en outre séparés par tous les Theridomys-ParatheridomysThalerimys, toutes ces espèces (clades $1 \& 2$ ) sont pour l'instant attribuées provisoirement au même genre Blainvillimys. Pour le clade des formes éocène $+\langle B »$. langei on utilisera des guillemets («Blainvillimys ») pour souligner le questionnement et l'état provisoire de cette dénomination. Ces deux clades correspondent à des grades dans l'évolution de l'antéroconide et de la fréquence du sI sur les molaires inférieures, de l'hypsodontie et de la téniodontie avec toutes les modifications que cela entraîne, notamment sur la longueur des flexus et flexides. La définition d'un nouveau genre pour le clade des formes éocènes impliquera l'examen d'un matériel plus abondant pour les espèces éocènes notamment et également une révision morphologique plus détaillée des espèces des deux clades.

Parmi les espèces éocènes, «B. » euzetensis est définie seulement par le type (P4-M1) d'Euzet (Gard ; MP17), ce qui n'a pas permis de l'inclure dans notre analyse cladistique. Il existe des populations provenant d'autres localités d'âges voisins de celui d'Euzet, mais il n'est pas possible pour l'instant d'affirmer qu'il s'agit bien du même taxon qu'à Euzet. Dans le matériel de Fons 4 (Gard) attribué à Theridomys euzetensis (Hartenberger, 1973 b), il semble qu'il y ait plusieurs genres représentés (probablement Elfomys, ? Pseudoltinomys et "Blainvillimys »). Ceci pose problème lorsque les mesures originales données pour Fons 4 sont utilisées pour comparer le matériel d'Eclepens B par exemple (Hooker \& Weidmann, $2000: 50)$ et l'attribuer à l'espèce euzetensis. Le type de «B. » euzetensis (P4-M1) montre que la P4 est un peu plus petite que la M1 ; le protolophe est rectiligne, attaché directement au protocône ; la mure est interrompue ; le mésostyle, gros sur P4, l'est moins sur M1; le mésolophe est long, s'abaissant vers l'extrémité de la préhypocrista. Le métalophe de M1, discontinu, s'attache au milieu de l'hypocône (Fig. 16). Ces caractères se retrouvent sur une partie des dents d' «Isoptychus » euzetensis d'Eclepens B. Ces dernières sont cependant légèrement plus grandes que le type, la P4 y étant aussi plus longue que la M1. Il sera donc nécessaire de réviser ces « Blainvillimys » anciens.

L'espèce B. varians a été créée par Thaler (1969) pour désigner les spécimens de Blainvillimys de Montalbán (Teruel, Espagne) provenant de « deux points fossilifères distants 
d'une centaine de mètres et superposés » (MLB pour le site A et MLBS pour le site B, au dessus de A). C'est ce matériel, conservé dans les collections de Sabadell (Barcelone), avec quelques éléments supplémentaires dans celles de l'Université de Montpellier, qui a servi de base à la définition de Theridomys varians par Thaler. Le terme "varians " voulait décrire la variation observée de la fréquence du sI aux dents inférieures. Dans l'état des connaissances (Vianey-Liaud, 1972b), la mise en synonymie de l'espèce $T$. varians avec $T$. gregarius semblait possible, cette variation étant observée chez les deux espèces. La fréquence du sI de gregarius était alors estimée entre 10 et $70 \%$. En même temps était créée l'espèce Blainvillimys helmeri des Chapelins (Vaucluse) : dans la population-type, le sI est toujours présent sur les quelques dents peu usées et disparaît rapidement à l'usure car très peu profond ; l'obliquité des crêtes est plus forte que chez B. gregarius (Vianey-Liaud, 1972b). Cependant, l'effectif de la population des Chapelins est faible. Plus tard, ont été attribuées à $B$. helmeri des populations plus abondantes, comme celles de Pech-Crabit ou Itardies (Quercy) (e.g., Vianey-liaud, 1998 : 256), en discriminant plus finement les espèces en matière de fréquence du sI. En même temps, des analyses morphométriques permettaient d'exprimer les variations de téniodontie et d'hypsodontie (e.g., Vianey-Liaud, 1998 : tab. 3 ; Vianey-liaud \& Schmid, 2009). Hugueney (1994) faisait remarquer avec raison que si la population de Montalbán et celle des Chapelins représentaient un même grade évolutif, il faudrait mettre $B$. helmeri en synonymie avec $B$. varians. Cependant, « comme il n'était pas impossible que les formes espagnoles aient subi une évolution autonome » (Hugueney, 1994), B. helmeri était conservé pour l'espèce des Chapelins.

De nombreuses fouilles ont été effectuées par l'équipe de Saragosse sur les affleurements de Montalbán (Freudenthal et al., 1990). Le site de Montalbán 3 a fourni neuf niveaux fossilifères superposés et trois communautés fauniques différentes, caractérisées d'après Freudenthal et al. (1990) par des espèces de rongeurs différentes. Des listes de rongeurs ont été établies pour 20 sites dans cette zone, dans lesquels Blainvillimys est identifié soit comme B. gregarius, soit comme B. varians (Freudenthal, 1997). Par exemple, à MLB $9, B$. gregarius est identifié sur la base d'une fréquence de $60 \%$ de sI. Or une telle fréquence correspond à celle de $B$. helmeri (e.g., Vianey-Liaud \& Schmid, 2009). Il faudrait comparer la population-type de B. varians (Thaler, 1969 : MLB et MLBS) avec celle de MLB 1D, cette dernière montrant d'après Freudenthal une plus forte proportion de sI que celle donnée par Thaler, alors que ces deux localités correspondraient au même site d'après Freudenthal. En fait, toute solution à cette question de l'attribution spécifique des Blainvillimys (et aussi des Theridomys et Pseudoltinomys) de Montalbán, nécessiterait une analyse morphologique et morphométrique de tous les loci dentaires des différentes populations et leur comparaison avec ceux des espèces bien connues d'autres zones géographiques (e.g., Les-Chapelins, Valbro, Cavalé, Pech-Crabit, Itardies). Il serait ainsi nécessaire de prendre en compte non seulement la fréquence des sI, mais aussi l'ensemble des caractéristiques morphologiques et de leur variabilité, pour que la synonymie de $B$. helmeri avec $B$. varians puisse être actée ou non. Par ailleurs, le fait que les deux espèces $B$. gregarius et $B$. varians apparaissent en succession stratigraphique à Montalbán apporterait des données supplémentaires pour la caractérisation de ces deux grades évolutifs.

Blainvillimys avus, connue à ce jour de deux localités d'Aquitaine du niveau MP24, représente une autre lignée, dont l'origine et le devenir sont encore incertains. La variabilité des dents inférieures, avec communication possible du sIV avec le sinuside, comme chez Toniodus, l'avait fait placer dans ce dernier genre par Stehlin \& Schaub (1951). Cependant les caractéristiques de la dent déciduale inférieure sont très différentes (cf. Vianey-liaud, 1998 : fig. 2a). Selon les résultats de notre analyse AG (Fig. 7B), cette espèce, aux caractères originaux, se place au sein des Blainvillimys oligocènes (clade 2), proche des B. blainvillei et distincte des Toniodus. Blainvillimys avus demeurera donc dans ce genre pour l'instant.

Caractères dentaires. Cf. Vianey-Liaud (1998 : 256), VianeyLiaud \& Schmid (2009).

Histoire paléogéographique. La plus ancienne espèce connue de Blainvillimys ( ( $B$. » euzetensis) est signalée dans le Bartonien supérieur du sud de la France et peut-être dans le Jura Suisse. Sans quitter cette zone géographique, leur aire de répartition s'étend ensuite jusqu'au sud de l'Angleterre, partie la plus septentrionale de leur territoire et jusqu'en Espagne, partie la plus méridionale avec «B. » pseudosiderolithicus et «B. " civracensis (MP18), «B. " rotundidens à la fin du Priabonien (MP19-20) et au début du Rupélien avec «B. » langei et B. gregarius (MP21-22). Blainvillimys est alors absent de Belgique et du sud-ouest de l'Allemagne, où seulement $B$. heimersheimensis est documenté plus tard (MP24). Blainvillimys blainvillei (MP25) n'est présent qu'en France (Auvergne, Quercy, Languedoc, Provence). Dans la molasse Suisse, le genre n'est plus représenté avant le début du Chattien (B. stehlini : MP25/MP26). L'extension temporelle du genre est très longue, près de $12,5 \mathrm{Ma}$; celle de la lignée $B$. gregarius- $B$. stehlini est de près de $5,5 \mathrm{Ma}$.

Tribu Archaeomyini Schlosser (in Stehlin et Schaub, 1951)

D’après Mödden (1993), cette tribu est caractérisée par un seul et unique caractère : la disparition du sIII sur les dents inférieures, qui va de pair avec la profonde fosse qui divise ces dents en deux parties. Cette tribu rassemble ainsi les genres Archaeomys et Protechimys. Depuis, des espèces plus anciennes (MP23/24 et MP/24) de Protechimys ont été reconnues, chez lesquelles le sIII est présent, le sinuside ne divisant pas profondément la dent (Vianey-Liaud, 1998, 2015 : 457-458). Les caractères qui individualisent la tribu apparaissent au nœud $\mathrm{W}$ de l'AG (Fig. 7B). Les deux genres montrent l'acquisition parallèle de plusieurs traits, liés au développement de la téniodontie et de l'hypsodontie, convergents aussi avec ceux rencontrés chez les Blainvillimys oligocènes (cf. p. 46). Le genre Toniodus est le groupe-frère de cet ensemble. Suivant la topologie des cladogrammes AG et AO (Figs 7B et 9), ce genre peut alors s'intégrer dans la tribu Archaeomyini.

\section{Genre Toniodus Pomel, 1853}

Espèce-type. Toniodus curvistriatus Pomel, 1853.

Caractères du genre. cf Vianey-Liaud, (1998 : 271-273).

Espèces attribuées, localités et niveaux biochronologiques. Toniodus curvistriatus Pomel, 1853, La-Sauvetat (A.C. Auvergne, France), Coulou (Lot, Quercy, France), Balm (Jura Suisse), MP22 ; Tæniodus aff. curvistriatus, Gas et Maintenon (Bassin-de-Paris, France), Saint-Martinde-Castillon (Vaucluse, France), MP23/24 ; Toeniodus hexalophodus Bahlo, 1975, Heimersheim (Bassin-de-Mayence, Allemagne), MP24. 
Caractères dentaires. Le degré d'hypsodontie et le degré de téniodontie des espèces du genre Toniodus sont moindres comparés aux Archaeomyini. Sur les DP4s de Toniodus, l'antésinus et la mure sont absents. Le SI est relativement profond sur les P4s et le SIV est oblique. L'hypocône est étiré en crête oblique anguleuse. Le s0 des dp4s est absent chez T. curvistriatus, présent chez T. hexalophodus, et leur sI est complètement clos. Ce sI est presque toujours présent sur les m1-2s. Le mésostylide, fort, est connecté au mésolophide + crête postmétacristide. Le sIV communique profondément avec le sinuside du fait de l'absence de préhypocristide. L'entolophide est connecté au néomésoconide ou à l'ectolophide.

Remarque. On note un accroissement modéré de la taille entre les deux espèces ainsi que l'allongement du SIV et du sI. Le s0 (dp4) et le SV sont variablement développés.

Histoire paléogéographique. Ce genre n'apparaît qu'au début du Rupélien (MP22) en France (Quercy et Auvergne) et dans le Jura Suisse. Il est ensuite documenté du Bassin-de-Paris à la Provence (MP23/24). Il disparaît après sa dernière présence au sud de l'Allemagne (MP24). Sa durée de vie est d'un peu moins de 4,5 Ma.

\section{Genre Protechimys Schlosser, 1884}

Espèce-type. Protechimys gracilis Schlosser, 1884, A.C. Quercy, France, MP indet.

Diagnose du genre. cf. Vianey-Liaud (1998 : 261).

Espèces attribuées, localités et répartition biochronologique. Protechimys truci (Hugueney, 1994), St-Martin-de-Castillon (Vaucluse, France), MP23/MP24 ; Protechimys lebratierensis Vianey-Liaud, 1998, Lébratières 14 (Lot, Quercy, France), Vialenc (Cantal, France), MP24; Protechimys variabilis Vianey-liaud, 1998, Mas-de-Gaston (Lot, Quercy, France) MP24/25 ; Protechimys gracilis Schlosser, 1884, Mouillac (A.C., Quercy, France), Belgarric 1, Rigal-Jouet 1 (Tarnet-Garonne, Quercy, France), St-Martin-de-Casselvi (Tarn, France), Burgmagerbein 2 (Jura Souabe, Allemagne), Talent 7 (Molasse Suisse), MP25 ; Protechimys blainvillei (Lavocat, 1951), « Antoingt » (A.C., Auvergne), MP indet., Rigal-Jouet 1 et Garouillas (Tarn-et-Garonne et Lot, Quercy, France), StMartin-de-Casselvi (Tarn, France), Aubenas-les-Alpes (Alpesde-Haute-Provence, France), MP25 ; Protechimys mayoi Mödden, 1993 (A.C., Quercy) MP indet. ; Protechimys major Schlosser, 1884, Mouillac (A.C., Quercy) MP indet., Mas-dePauffié, La-Devèze, Espeyrasse (Quercy, France), Saint-Privatdes-Vieux (Gard, France), Les-Milles (Bouches-du-Rhône, France), Mumliswyl-Hardberg, Oensingen, Aarwangen, Talent 1 (Molasse Suisse), MP26.

Caractères dentaires. cf. Nœud W1 (Fig. 7B; p. 25) ; cf. Vianey-Liaud (1998, 2015).

Remarque. Tous les Protechimys, comme les Archaeomys, ont un palais relativement plus long à l'avant, puisque les foramens palatins antérieurs (fpa) atteignent seulement le bord antérieur de DP4 ou P4, alors qu'ils se prolongent jusqu'à la partie postérieure ou au milieu de DP4 et P4 chez les Blainvillimys.

Histoire paléogéographique. Ce genre a une extension temporelle relativement courte, environ 3,5 Ma. Il se différencie au cours du Rupélien supérieur et au tout début du Chattien dans la partie sud de la France (MP23/24 et MP24). Il étend son aire de répartition jusqu'au Jura Souabe et en Suisse (MP25MP26). Il disparaît ensuite.
Genre non nommé «Archaeomys/Blainvillimys » cf. VianeyLiaud (2015 : 464, 467-468)

Espèces attribuées, localités, niveaux biochronologiques. Archaeomys/Blainvillimys sp., Mas-de-Pauffié et La-Devèze (Lot, Quercy, France), St-Privat-des-Vieux (Gard, France), Les-Milles (Bouches-du-Rhône, France), MP26 ; Archaeomys/ Blainvillimys huerzeleri Thaler, 1966 (= Bl. geminatus Thaler, 1966), St-Menoux (Allier, France), Oensingen, Boningen, (Molasse Suisse), MP26-MP27 ; Archaeomys/Blainvillimys robustus (Lavocat, 1951) St-Henri/St-André (Bouchesdu-Rhône, France), MP26, Gaimersheim (Jura Souabe, Allemagne), Aarwangen (Molasse Suisse), MP26-MP27.

Remarques taxonomiques. L'étude menée sur les Archaeomyini de Mas-de-Pauffié, Les-Milles et St-Henri/ St-André a permis de souligner les ambiguïtés dans la dénomination des populations du Chattien inférieur (MP26-MP27) (Vianey-liaud, 2015). En effet, ces formes attribuées soit à Blainvillimys soit à Archaeomys sont généralement mal représentées dans les localités fossilifères, ce qui limite la connaissance de tous les loci dentaires et de la variabilité de leur morphologie. Cette instabilité taxonomique vient du fait que leurs dents inférieures ont un patron de type Archaeomys (sI, sIII et sIV absents ; sinuside traversant toute la dent et sII ouvert lingualement). Par contre, contrairement aux Archaeomys typiques du Chattien supérieur, les petits synclinaux externes - SI et SII - des prémolaires et des molaires supérieures sont présents chez ces formes, comme chez les Blainvillimys les plus dérivés (i.e., blainvillei et stehlini). En attendant la révision indispensable de toutes ces formes et la mise en œuvre de méthodes morphométriques à même de discriminer, éventuellement, des dents inférieures aussi semblables, ces espèces ont été intégrées en tant que telles dans la présente analyse cladistique.

Ces Archaeomys/Blainvillimys sortent en groupes frères des Archaeomys s.s. séparés des Blainvillimys par les Protechimys et les Toniodus. Cette topologie appuie donc leur inclusion dans le genre Archaeomys. Ces trois espèces d'Archaeomys/ Blainvillimys sont ainsi relativement éloignées (nœud W2 ; Fig 7B) des Blainvillimys dans la topologie (nœud V1). Les patrons de leurs dents jugales supérieures et inférieures sont très proches de ceux des Blainvillimys les plus évolués, beaucoup plus proches d'eux que de celui des Toniodus par exemple. Comme souligné dans l'analyse des caractères aux nœuds $\mathrm{V}$ et $\mathrm{W}$, les parallélismes constatés sont nombreux.

Pour l'instant, nous conservons provisoirement la double dénomination générique de ces trois espèces, dans la mesure où leurs contours demandent à être mieux définis. Elles sont en effet très rares dans les gisements (e.g., Vianey-Liaud, 2015).

Caractères dentaires. $\mathrm{Cf}$. ci-dessus.

Histoire paléogéographique. Chronologiquement ces trois espèces se situent d'une part entre les derniers Blainvillimys (B. stehlini) + Protechimys (P. major) (MP26) et, d'autre part, les premiers Archaeomys (MP27). Géographiquement, elles se rencontrent d'abord du sud de la France au Jura Suisse, et leur aire de répartition s'étend ensuite jusqu'au Jura Souabe.

Genre Archaeomys de Laizer \& de Parieu, 1839

Espèce-type. Archaeomys arvernensis (de Laizer \& de Parieu, 1839), Coderet (Allier, France), MP30. 
Espèces attribuées, localités, niveaux biochronologiques. Archaeomys ehrensteini Mödden, 1993, Ehrenstein 7 et Burgmagerbein 1 (Jura Souabe, Allemagne), Wynau (Jura Suisse), MP27; Archaeomys quercyi Mödden, 1993, Pech-Desse (Lot, Quercy, France), Gaimersheim (Jura Souabe, Allemagne), MP 28a ; Archaeomys intermedius Vianey-Liaud, 1979, Pechdu-Fraysse (Lot, Quercy, France), Gaimersheim (Jura Souabe, Allemagne), MP 28b ; Archaeomys helveticus Vianey-liaud, 1977, Rickenbach et Fornant 6 (Jura Suisse), Burgmagerbein 6 (Jura Souabe, Allemagne), Pech-du-Fraysse (Lot, Quercy, France), MP28b-MP29 ; Archaeomys arvernensis de Laizer $\&$ de Parieu, 1839, Coderet (Allier, France), Rickenbach (Jura Suisse), MP29 - MP30 ; Archaeomys laurillardi Gervais, 1852, A.C., Auvergne (Cournon ou Pérignat, Romagnat), Coderet (Allier, France), Küttigen (Jura Suisse), MP30.

Caractères dentaires. (Figs. 6, 16 et 20) La morphologie des molaires inférieures est très homogène au sein des Archaeomys et c'est pour cette raison que ces dents ont été peu prises en considération pour la systématique des espèces au sein du genre (e.g., Mödden, 1993). Dès les premiers stades d'usure, toute trace de sI disparaît des molaires. On ne distingue que trois lophides, séparés par le sII, ouvert lingualement, puis le sinuside, étendu jusqu'au bord buccal, dont il n'est séparé que par une mince jonction des extrémités linguales des lophides médian et postérieur sur les dents usées. On observe des différences d'angulation et d'obliquité de ces lophides d'une espèce à l'autre, qu'il faudrait caractériser morphométriquement, afin de mettre en œuvre des critères de distinction des espèces. Le même type de démarche devrait aussi concerner les dents supérieures, les dimensions linéaires étant sujettes à de fortes variations avec l'usure, du fait de l'inclinaison de plus en plus marquée des couronnes, allant de pair avec l'hypsodontie (Vianey-Liaud, 1979 ; Mödden, 1993).
Des singularités sont tout de même remarquées sur les dp4s de certaines espèces. A Ehrenstein 7 (MP27), Mödden distingue un morphotype (Mödden, 1993 : fig. 27a) qu'il attribue à $A$. ehrensteini, chez qui le s0 est clos lingualement, le sII est encore présent et ouvert également tout comme le sIV, le sIII traversant la dent. Sur la dp4 (o.c. fig. 27b), qu'il attribue à une autre espèce, $A$. huerzeleri (que nous avons considérée ici comme une forme d'Archaeomys/Blainvillimys), la morphologie est très proche de celle de $P$. major. Le sIV est présent mais réduit, le sinuside et aussi le sII + antésinuside sont traversants, le métaconide est proéminent et le sI s'ouvre lingualement. D'ailleurs, les DP4s (o.c. fig. 28c) montrent aussi comme celles de $P$. major un sinus traversant, mais l'antésinus est plus long, le S0 peut être un peu plus développé et un SV est présent. Les autres DP4s déterminées comme A. ehrensteini ont une mure et un SII présents. Mödden figure deux M1-2s, l'une attribuée à $A$. ehrensteini montre un petit SII, un SIII et pas de SIV, tandis que celle qu'il attribue à huerzeleri, plus téniodonte, porte un petit SII, un long SIII et un SIV bien individualisé. Le type d'A. huerzeleri de Boningen a un SIV mais pas aussi développé. Les dp4s connues de toutes les autres espèces montrent quatre lophides dont les moitiés linguales font une nette angulation avec les moitiés buccales; le sI est fermé lingualement seulement sur les dents bien usées, sinon sur les dents jeunes, il est ouvert ; le sIV est présent seulement sur les dents peu usées. Les p4s n’ont que trois lophides, le sII est ouvert lingualement et le sinuside est traversant.

La distinction des taxons se fait surtout sur la présence/ absence des petits synclinaux buccaux SI, SII et SV sur les prémolaires et molaires supérieures (cf. Mödden, 1993 ; Mödden \& Vianey-Liaud, 1997); pour Mödden, la numérotation des synclinaux sur les DP4s est différente de celle employée ici (cf. Fig. 18), sur laquelle la position du paracône donne

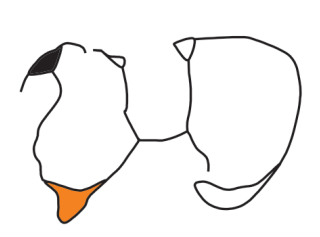

Protechimys truci

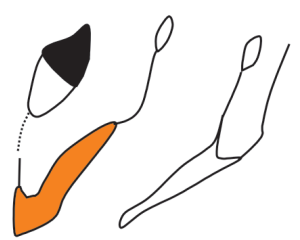

Protechimys major

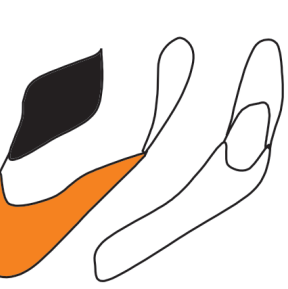

Archaeomys ? huerzeleri (Boningen)

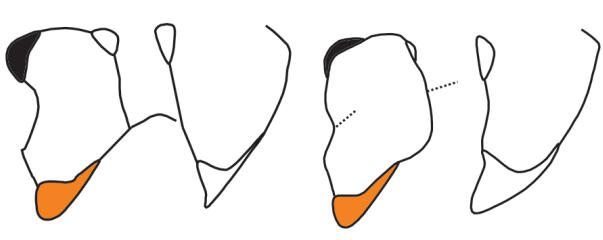

Pr. lebratierensis

Pr. variabilis

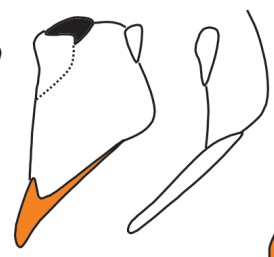

Pr. gracilis

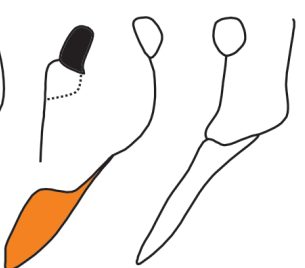

Pr. blainvillei

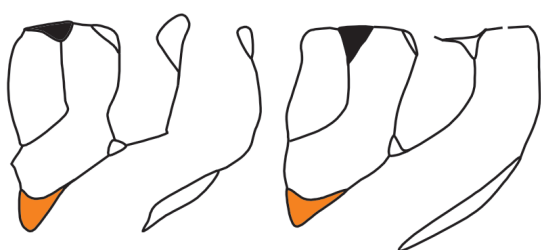

Tœniodus curvistriatus Tœniodus hexalophodus

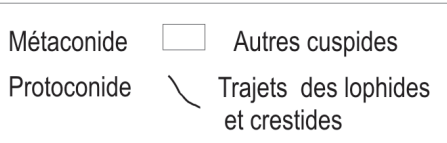
et crestides

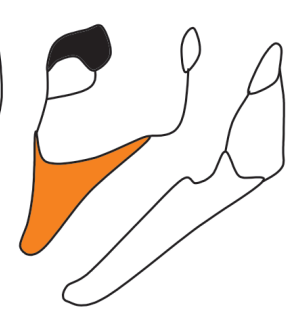

Archaeomys quercyi (Pech-Desse)

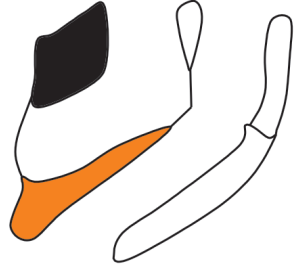

Archaeomys intermedius (Pech-du-Fraysse)

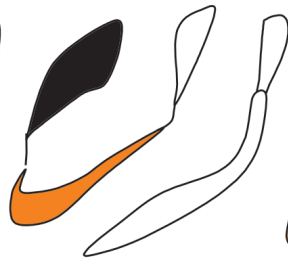

Archaeomys helveticus (Rickenbach)

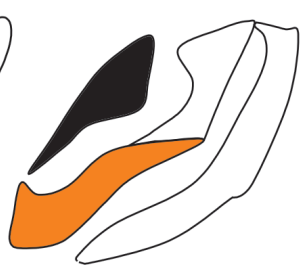

Archaeomys laurillardi (Coderet)

Figure 20. Schémas des m1-2s inférieures de diverses espèces d'Archaeomyini. 
la limite postérieure du SI ; pour Mödden, S0 = SI etc.. La prise en compte d'autres critères, notamment concernant les dents inférieures et les proportions des différents éléments des rangées dentaires, devrait permettre de mieux définir les espèces et de comprendre leurs relations.

Histoire paléogéographique. Les premiers Archaeomys (MP27) sont documentés dans le Jura Souabe et le Jura Suisse. Leur aire de répartition s'étend ensuite dans le centre et le sud de la France (MP28-MP30). L'extension temporelle du genre est relativement courte, autour de 2,5 Ma.

\section{LIGNÉES ÉVOLUTIVES}

Plus d'une trentaine de lignées évolutives, au sens des « chronospecies » de Simpson (1951), ont été identifiées au sein des Theridomyoidea, dont certaines ont été mentionnées ci-dessus (e.g., Schmidt-Kittler, 1971 ; Vianey-Liaud \& Ringeade, 1993 ; Vianey-Liaud, 1998). Ces lignées sont constituées de la succession temporelle d'espèces bien définies par leur morphologie. Du fait du processus de l'évolution, chacune diffère de celle qui la précède dans le temps par de petites modifications morphologiques et/ou dimensionnelles, ces différences n'altérant pas de façon majeure leur schéma dentaire commun : ainsi peut-on proposer un lien de parenté entre elles. Par contre, l'accumulation de ces changements orientés pourra conduire à des différences notables entre les stades évolutifs extrêmes d'une lignée, à l'issue d'un laps de temps plus ou moins long (e.g., pour la lignée des Issiodoromys, entre I. medius, environ 32 Ma et I. bransatensis, environ 23 Ma).

Comment a-t-on pu individualiser ces lignées et reconnaître des tendances évolutives?

\section{Méthodologie}

1- La méthode de recherche des relations de parenté entre les espèces de théridomyoïdes a été contrainte par le contexte particulier des localités fossilifères à Theridomyoidea. En effet, celles qui livraient des fossiles en abondance étaient des remplissages karstiques, notamment en Quercy (France), dans le Jura Suisse ou le Jura Souabe (Allemagne). Ce fut le cas en particulier pour les espèces de Theridomyidae depuis l'Eocène supérieur jusqu'à la fin de l'Oligocène. Or les remplissages karstiques, qui ont fourni nombre de ces rongeurs, ont un inconvénient important, la difficulté de les placer dans un contexte temporel sans avoir à s'appuyer sur une analyse de leur contenu fossilifère. Cette difficulté résulte de l'absence d'un contexte stratigraphique qui autorise une corrélation avec l'échelle GTS, faute de relations géométriques avec des niveaux marins ou à l'absence de minéraux qui permettent des datations radiométriques. Le second obstacle vient des gisements eux-mêmes, très peu de ces riches gisements karstiques renfermaient exactement les mêmes espèces de théridomyoïdes que ceux connus dans des gisements de bassins, que l'on avait pu, pour la plupart, corréler plus ou moins facilement à l'échelle globale. Alors, comment dater ces gisements karstiques de façon à en établir la succession temporelle afin de restituer l'évolution des espèces et les changements fauniques ? Le renouvellement faunique connu sous le nom de « Grande Coupure » en Europe (depuis Stehlin, 1909 ; e.g., Hartenberger, 1973 ; Vianey-liaud, 1979 ; López \& Thaler, 1985 ; Hooker, 2010) apporte un repère essentiel en permettant de situer les gisements quercinois dans
l'Eocène (présence de primates diversifiés notamment) ou dans l'Oligocène (arrivée massive de migrants asiatiques de tous les ordres de mammifères). Pour aller plus loin, il convenait d'utiliser au mieux, avec l'ensemble de la faune de chaque gisement, les fossiles les plus abondants que sont les rongeurs théridomyoïdes, tout en évitant tout possible raisonnement circulaire

2- Des milliers de restes dentaires de théridomyoïdes ont permis d'étudier chaque population fossile comme le font les biologistes qui s'intéressent aux petits mammifères, en ne se limitant pas à la description de quelques éléments dentaires, mais en analysant notamment toute leur variabilité. Comme l'actualité portait au moment de ces études sur la controverse évolution graduelle versus équilibres ponctués (e.g., Elredge \& Gould, 1972), il s'agissait de savoir si la documentation paléontologique soutenait l'une ou l'autre des théories. Bien sûr, le pas de temps de la paléontologie en général et le degré de finesse des analyses morphologiques n'autorisent pas à appréhender la réalité intrinsèque des mécanismes évolutifs. En effet, la durée de vie d'une espèce de rongeur fossile est au minimum de 250000 ans (Aguilar \& Michaux, 1987), alors que les changements se font à l'échelle des individus et des générations. Il ne permet pas plus d'établir une « généalogie » directe des espèces. C'est l'image enregistrée dans les caractéristiques différentes des espèces se succédant dans le temps qui est décryptée (e.g., Thaler, 1983 ; Vianey-Liaud \& Michaux, 2003), les changements d'une espèce à l'autre pouvant apparaître comme graduels ou non. Il était indispensable d'analyser des successions d'espèces à partir de faunes dont la succession est solidement établie, aussi rapprochées que possible, pour proposer des relations de parenté étroites entre les différentes espèces supposées constituer une "lignée évolutive » (Simpson, 1951). Quoique cette proposition apparaisse étrange en matière d'analyse cladistique, elle s'est avérée à l'usage parfaitement heuristique, une fois que l'on a bien posé les limites rappelées ci-dessus, tant du point de vue des mécanismes évolutifs que des relations de parenté (longue durée de vie des espèces paléontologiques; pas de généalogie directe).

Dans le contexte ci-dessus, nous avons eu la chance de trouver dans les nouveaux gisements quercinois des espèces qui se ressemblaient beaucoup entre elles, mais qui différaient par de petits écarts dans la taille, la hauteur des couronnes dentaires, des éléments du schéma occlusal, etc. La ressemblance globale, exprimée dans les caractères morphologiques et biométriques de la couronne dentaire, a permis de poser les bases des premières hypothèses de relations de parenté. Ensuite, des hypothèses de tendances évolutives ont été faites en prenant comme fil conducteur le degré d'hypsodontie des espèces. En effet, les changements environnementaux qui marquent la fin de l'Eocène et l'Oligocène (Fig. 3), provoquant notamment l'ouverture des milieux dans le sud de l'Europe Occidentale et permettant l'arrivée de nouvelles espèces migrant depuis l'Asie notamment, ont été particulièrement déterminants pour le développement et la sélection de morphologies dentaires hypsodontes adaptées à un régime alimentaire abrasif. Ainsi des successions d'espèces d'hypsodontie croissante ont été proposées comme lignées évolutives, incluant parfois des taxons provenant de gisements dont la position stratigraphique était connue. En vérifiant que plusieurs lignées [de théridomyoïdés, d'autres rongeurs (Eomyidae, Cricetidae... ; e.g., Comte \& Vianey-Liaud, 1989), ou encore d'autres mammifères (e.g., 
Sudre, 1978, 1988 ; Blondel, 2001, 2005)] fournissaient le même signal de polarité temporelle, en y ajoutant les quelques repères stratigraphiques connus, les hypothèses de tendances évolutives ont été testées et vérifiées dans presque tous les cas. Par ailleurs, le suivi des transformations morphologiques au long de ces lignées, a donné une image graduelle de l'évolution qui les a affectées (e.g., Vianey-Liaud \& Michaux, 2003).

Il est bien sûr indispensable de prendre en compte les cas de possibles convergences morphologiques, sinon au risque d'être dans l'erreur si la faune associée aux théridomyoïdes est peu connue ou peu diversifiée. Cela fut le cas pour «Theridomys» bonduelli, d'abord considérée comme une forme intermédiaire entre « T. » aquatilis et T. major (Vianey-Liaud, 1972a et b) puis considérée ultérieurement comme lignée parallèle, plus ancienne que «T. » aquatilis - T. major (p. 40-41 et VianeyLiaud, 1989).

Dans ce contexte méthodologique, compte tenu de la quantité et de la qualité du matériel fossile concerné, la recherche sur les théridomyoidés s'est faite à côté du développement des méthodes phylogénétiques cladistiques qui ont connu leur épisode d'expansion à partir de la fin des années 1980. Les analyses de caractères morphologiques sur les rongeurs étaient encore peu formalisées dans l'état des lieux effectué lors du colloque «Evolutionary Relationships Among Rodents » (Luckett \& Hartenberger, 1985). Elles se sont ensuite (beaucoup) développées tandis que les biologistes moléculaires produisaient des phylogénies des rongeurs actuels et qu'il devenait possible de savoir dans quelle mesure les caractères morphologiques des mêmes rongeurs utilisés pour la classification les soutenaient. Mais les formes actuelles ne représentent qu'une part de la diversité totale de l'ordre des Rodentia depuis sa différenciation : des radiations manquent totalement et quelques riches radiations anciennes n'ont plus que quelques rares témoins survivants.

Les restes fossiles étant essentiellement des restes dentaires pour les rongeurs, il s'agissait de tester leur pertinence comme support d'analyses cladistiques. C'est ce qui a été réalisé, montrant tout l'intérêt des caractères dentaires pour la phylogénie des rongeurs au moment de leurs premières radiations, au cours de l'Eocène (e.g., Marivaux et al., 2004). Les convergences et parallélismes dans la réalisation d'un nombre limité de patrons dentaires sont légion chez les rongeurs. En restreignant les taxons considérés à ceux issus de leurs premières radiations, les accumulations d'homoplasies récurrentes ont pu être limitées. Suivant cette approche et cette méthodologie, on peut envisager à présent de comparer les résultats obtenus par la méthode des lignées évolutives, avec ceux d'une analyse cladistique fondée essentiellement sur des caractères dentaires.

\section{Lignées évolutives : les acquis et les nouvelles questions.}

Dans les parties précédentes (Analyse des caractères aux nœuds et Systématique), nous avons pu considérer plusieurs de ces lignées de Theridomyoidea.

\section{Protadelomys, des lignées à reconsidérer}

Pour Hartenberger (1969) puis Escarguel (1998, 1997, 1999), les quatre espèces de Protadelomys ( $P$. maximini - $P$. alsaticus - P. cartieri - P. lugdunensis) documenteraient une lignée évolutive qui représenterait une succession temporelle dans l'intervalle MP13 à MP14. Pour Escarguel, la cinquième espèce, $P$. nievesae, de plus petite taille et plus ancienne
(Peláez-Campomanes, 1995 ; MP11/12), témoignerait d'une autre lignée évolutive, propre à l'Espagne. Sur l'arbre obtenu dans notre analyse (Fig. 21), les espèces de Protadelomys ne se succèdent pas comme les hypothèses de lignées évolutives le proposaient. Ainsi notre analyse fournit un arbre qui ne suit pas l'ordre chronologique supposé puisque les deux espèces les plus anciennes $P$. nievesae (MP11/12) et $P$. maximini (MP13/14) sont placées dans le même clade terminal. $P$. cartieri (MP14) émerge la première puis $P$. lugdunensis (MP14), puis P. alsaticus (MP13). Escarguel représente chacun des quatre taxons de Protadelomys (P. nievesae non compris donc) par un "morphodontogramme » qui traduit le rapport de forme et de taille relative des quatre dents de la rangée dentaire (Escarguel, 1997). Ces dents sont la plupart du temps fossilisées isolément, indépendamment de tout os dentaire ou maxillaire. Les modifications de ces courbes et l'augmentation des coefficients mandibulaires moyens de $P$. maximini à $P$. lugdunensis sont interprétées en termes de degré d'évolution dans la lignée supposée. Deux espèces plus récentes de Protadelomys ont été signalées l'une à ChéryChartreuve (Aisne, France, Comte et al., 2012 : pl. 2r-w ; MP15/16) l'autre à Pontils 26 (Comte et al., 2012 : pl. 8t ; DP4, MP16). Chez la première, les tubercules sont bulbeux et le sinus marqué bien que court, comme chez $P$. lugdunensis et $P$. cartieri. Protadelomys alsaticus et $P$. maximini montrent tous deux une surface de l'émail plus fortement crestulée. De plus, chez ces deux espèces, l'ensemble préprotocrista - protocône endolophe constitue une arête comprimée bucco-lingualement, le sommet appointé du protocône émergeant peu et le sinus y est absent ou peu marqué. Protadelomys nievesae montre des tubercules saillants, l'endolophe relativement bas, le sinus très réduit, les conules forts et les crestules moins nombreuses que chez $P$. maximini.

Le cladogramme (Fig. 21) qui montre que les relations de ces espèces sont loin d'être solidement établies confirme la nécessité de revoir l'interprétation retenue jusqu'ici.

\section{Des lignées à mieux caractériser}

Les analyses phylogénétiques (AG ou $\mathrm{AE}$ ) montrent des regroupements cohérents des espèces de la plupart des genres de Pseudosciuridae tels Sciuroides, Pseudosciurus, Treposciurus ou Pseudosciurus, et soutiennent leur bien-fondé. Des questions se posent seulement pour les espèces attribuées à Tarnomys (cf. Systématique, p. 32).

Les études de ces genres n'ont fourni que peu de résultats en termes de lignées évolutives, en dehors des Pseudosciurus et Suevosciurus (Schmidt-Kittler, 1971). Pour ces derniers, cependant, peu ou pas de stades évolutifs ont été matérialisés par des dénominations spécifiques. Ainsi, la lignée des Pseudosciurus dure près de $4,5 \mathrm{Ma}$, mais seulement deux espèces sont nommées : Pseudosciurus prcecedens (MP18-MP19) et $P$. suevicus (MP20-MP23). Il en est de même pour les Suevosciurus : Schmidt-Kittler (1971) reconnaît deux lignées qui ont pour origine $S$. minimus (MP18-19), l'une conduisant vers $S$. fraasi, à présent identifiée entre MP18 et MP22, l'autre vers $S$. ehingensis, espèce beaucoup plus grande, présente de MP19 à MP25. Les relations entre les petites espèces de Suevosciurus ne sont pas résolues à l'issue de notre analyse cladistique ( $S$. ehingensis n'a pas été intégrée à l'analyse). Heissig (1987) a proposé une autre espèce, $S$. dehmi, " de taille intermédiaire entre $S$. minimus et $S$. fraasi », laquelle n'a pas été incluse ici du fait de la caractérisation sommaire de la 


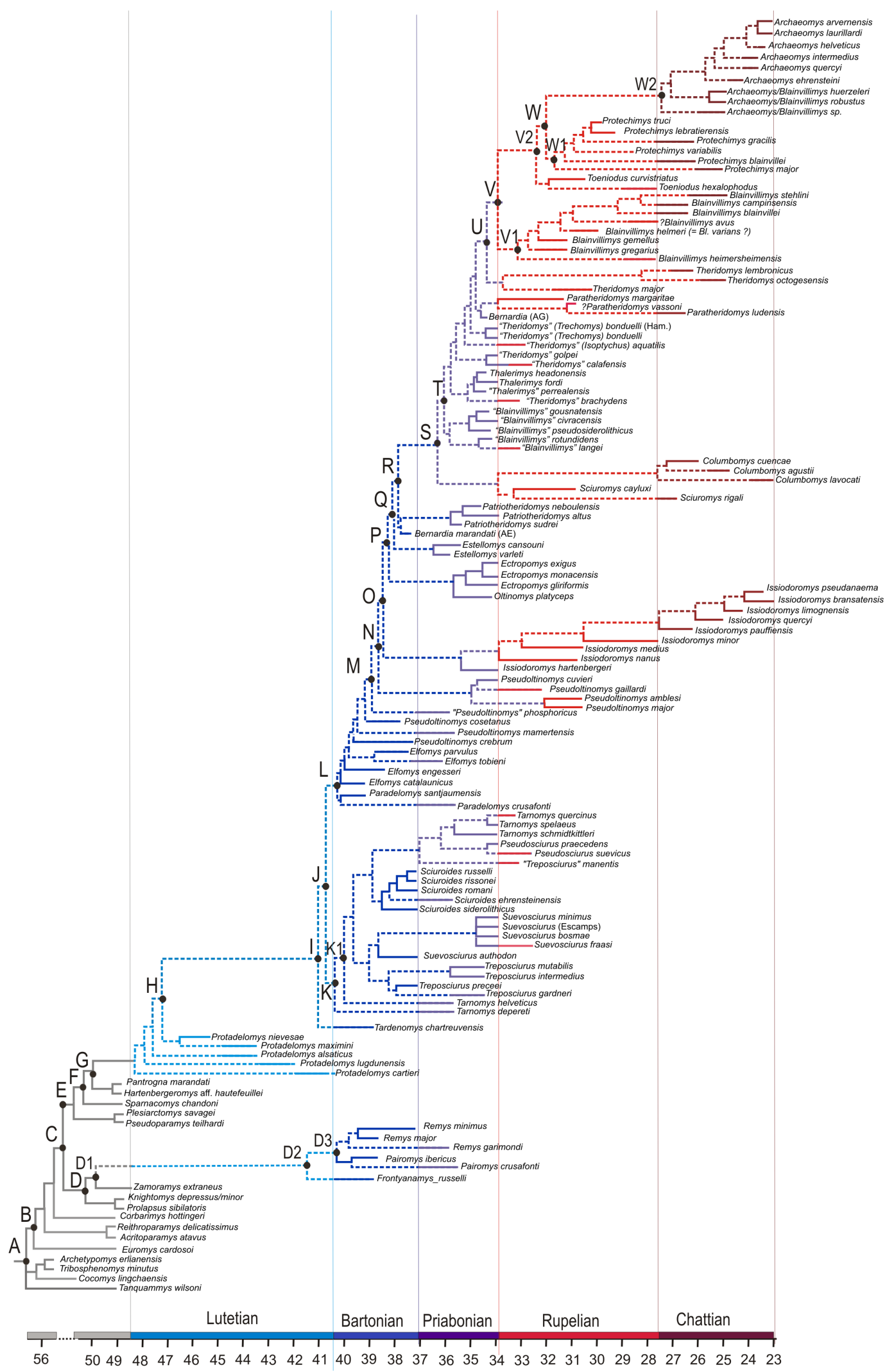


morphologie et de la taille. Un grade évolutif plus archaïque est Suevosciurus authodon (Hooker, 1986 ; MP16). Nos résultats phylogénétiques placent bien cette dernière en espèce-soeur de toutes les autres petites espèces du genre. La révision des populations les plus abondants des espèces à longue répartition temporelle de Suevosciurus et Pseudosciurus devrait permettre de mieux définir les modifications évolutives et notamment au niveau de l'importance des dents déciduales.

La dichotomie des Treposciurus place d'un côté les espèces les plus anciennes (T. gardneri et $T$. precei), qui sont représentées par du matériel abondant et bien connu, et de l'autre côté les plus récentes (T. intermedius et T. mutabilis), beaucoup moins bien documentées. Pour les Sciuroides, S. romani est placée en espèce-soeur du clade $S$. russelli (MP16) - S. rissonei (MP16) et à un degré hiérarchique plus avancé que celui de S. ehrenteinensis (MP18) ou S. siderolithicus (MP15/16). Pour ces deux genres seuls les taxons $S$. russelli et $S$. rissonei sont relativement bien documentés.

\section{Des portions de lignées consolidées, des éclaircissements taxonomiques à apporter}

\section{- Les lignées de Columbomyinae}

Une lignée évolutive est postulée à partir des espèces successives de Sciuromys (S. cayluxi, MP22;S. quercyi, MP25) jusqu'à Columbomys (Thaler, 1996). Limitant l'espèce S. quercyi au lectotype (A.C., Quercy, MP indet.), Mödden (1993) renomme $S$. rigali les populations de Belgarric 1 et Rigal-Jouet 1 (MP25). Hugueney et al. (1992) décrivent deux nouvelles espèces de Columbomys d'Espagne, $C$. cuencae (MP26) et C. agustii (MP28). Sur la base de leurs taille et hypsodontie, notamment celle de $C$. cuencae, ils en déduisent que Sciuromys ne peut être directement lié à Columbomys; les espèces de la péninsule ibérique représenteraient une lignée endémique, conservatrice, distincte de celle qui aurait pu conduire à $C$. lavocati, espèce connue dans le sud de la France. Depuis, deux dents de plus grande taille et plus hypsodontes que $S$. rigali ont été récoltées dans le gisement d'Espeyrasse (Lot, Quercy, MP26). Si la molaire inférieure se rapproche des plus grandes $\mathrm{m} 2 \mathrm{~s}$ de $S$. rigali, avec un antésinus moins profond que celui de $C$. cuencae, la M1-2 (2,1 x $2.0 \mathrm{~mm}$ sur la surface d'usure) est plus grande que $S$. rigali.

Ainsi, ces genres sont présents mais peu représentés dans les gisements méridionaux de l'ouest européen. La variabilité des espèces, hormis celles des Sciuromys, est mal connue et les arguments manquent encore pour soutenir ou réfuter une (ou deux) lignée(s) Sciuromys - Columbomys. Le signal donné par la topologie de l'AG (ainsi que AO) indique que les deux genres constituent un clade, avec Sciuromys comme genre frère du clade Columbomys lavocati - Columbomys cuencae $+C$. agustii. Les espèces successives de Sciuromys montrent un accroissement de l'hypsodontie. Entre Columbomys cuencae et les autres espèces de Columbomys, en plus d'une hypsodontie croissante, s'ajoute un accroissement de la taille des dents et aussi de la longueur des prémolaires par rapport aux molaires.

- Une portion de lignée de Theridomys.

La topologie de AG (Fig. 7B) ne soutient que le seul clade des Theridomys les plus dérivés (T. major, T. lembronicus et $T$. octogesensis). Ces espèces constituent les 3 derniers stades de la lignée évolutive des Theridomys, telle que définie antérieurement (depuis Vianey-Liaud, 1972a et b). Cette lignée se différencie notamment de celle des Blainvillimys par une taille plus importante des dents.

Toutes montrent une forte fréquence du sI aux dents inférieures et une épaisseur de l'émail constante sur le pourtour des flexus et flexides. Les tendances évolutives observées sont l'augmentation de la taille, de l'hypsodontie et de la téniodontie, avec l'acquisition d'un s0 à toutes les dents inférieures et un SV à toutes les dents supérieures chez T. octogesensis. Il est possible que les espèces « T. » aquatilis, T. golpei, $T$. calafensis et $T$. sp. de Ruch et Pouquette (MP22, Aquitaine ; e.g., Brunet et al., 1977) puissent se rattacher à cette lignée, avec, éventuellement, des fluctuations de la taille en fonction de leur origine géographique. La discussion précédente (cf. Systématique, p. 40-41) a montré les incertitudes concernant les affinités de plusieurs taxons tels que «T. pourtant bien documenté, ou «T. . brachydens avec les trois espèces ci-dessus. La taille absolue n'a bien sûr pas été prise en compte dans notre analyse des caractères.

\section{Des lignées confortées}

\section{- La lignée de Patriotheridomys et l'énigme de Bernardia marandati résolue?}

Les trois espèces du genre Patriotheridomys proviennent de faunes placées avec précision dans l'échelle des niveaux MP. Dans la topologie obtenue elles constituent un clade. La plus ancienne, $P$. sudrei, est l'espèce-sœur des deux autres. Cette représentation topologique appuie donc la lignée proposée. Entre les trois espèces, on note successivement une augmentation relativement faible de la taille générale, mais plus forte de l'hypsodontie, surtout entre $P$. sudrei et $P$. neboulensis. Les DP4s et dp4s s'allongent relativement plus, avec renforcement antérieur du dédoublement de l'antéroconide mésio-lingual (= métaconide ?) et renforcement du pré-protoconide dédoublé du côté buccal. Les molaires inférieures, dépourvues de sI chez $P$. sudrei et $P$. neboulensis, l'acquièrent sur une partie d'entre elles chez $P$. altus (environ $32 \%$ des $\mathrm{m} 1-2 \mathrm{~s}$ ).

Sur l'analyse réduite aux espèces éocènes (AE ; Fig. 8), réduisant a priori ainsi les homoplasies dues au grand nombre de lignées hypsodontes oligocènes, Bernardia marandati (MP16), une espèce qui apparaissait jusqu'ici très isolée au sein des théridomyidés, se retrouve à proximité du genre Patriotheridomys (MP18-MP20). Elle constitue l'espèce soeur $\mathrm{du}$ groupe Patriotheridomys - Blainvillimys - Thalerimys Theridomys. Tous les loci dentaires n'étant pas représentés, la proximité hiérarchique de Bernardia et de Patriotheridomys ne peut être complètement argumentée. La très forte hypsodontie de Bernardia en ferait le représentant ancien d'une deuxième lignée de Patriotheridomyinae.

\section{- Les lignées d'Issiodoromyinae}

De nombreuses analyses biométriques et morphométriques ont été réalisées sur un grand nombre de populations (population = assemblage fossile provenant d'une seule localité et attribué à une espèce) oligocènes appartenant au genre Issiodoromys. Ces

Figure 21. Arbre de consensus strict issu de l'analyse de tous les taxons considérés (AG), replacé sur l'échelle géochronologique (GTS). La convention des couleurs est : gris pour l'Eocène inférieur, bleu moyen pour le Lutétien, bleu foncé pour le Bartonien, violet pour le Priabonien, rouge pour le Rupélien, brun pour le Chattien. 
analyses ont permis de bien caractériser les espèces, de taille et d'hypsodontie croissante. Les schémas occlusaux varient beaucoup avec l'âge (donc avec l'usure). Des paramètres ont alors été définis et mis en œuvre pour lier schémas et hauteur de couronne, offrant une représentation formelle des populations (e.g., Vianey-Liaud \& Schmidt-Kittler, 1987). Parallèlement, il est apparu vain de comparer les moyennes des rapports d'hypsodontie de différentes populations. En effet, en fonction de la taphonomie, les représentations des classes d'âge dans les assemblages fossiles par des dents plus ou moins usées et plus ou moins hautes ne sont pas les mêmes et influent beaucoup sur ces moyennes. Il est bien plus juste de représenter l'hypsodontie de ces populations par l'amplitude de variation de la hauteur des dents, la hauteur maximum mesurée sur les dents à peine usées des différents loci étant alors pertinente, ou par les paramètres morphométriques de caractérisation des indentations de la surface en fonction de la hauteur de la couronne.

L'un des changements majeurs dans l'évolution morphologique tout au long de la lignée principale des Issiodoromys est l'accroissement de plus en plus marqué de la taille des prémolaires déciduales, tandis que les prémolaires définitives demeurent plus petites que les molaires. Il s'agissait vraisemblablement de laisser aux juvéniles une surface de mastication fonctionnelle suffisamment longue, alors que les deux premières molaires, hypsodontes et peu usées, n'offrent qu'une faible surface. Ce n'est qu'avec l'usure croissante, l'âge aidant, que les molaires montrent une table d'usure conséquente, tandis que les prémolaires définitives remplacent les dents déciduales. Ce phénomène est d'autant plus marqué que les dents sont plus hypsodontes et que la courbure des fûts dentaires augmente (e.g., Vianey-Liaud et al., 1995 : figs. 7 et 12 ).

Ainsi a été définie une lignée qui va d'Issiodoromys medius à $I$. bransatensis, selon des rythmes variables (Vianey-Liaud, 1976 ; Schmidt-Kittler \& Vianey-Liaud, 1987 ; VianeyLiaud \& Schmidt-Kittler, 1987 ; Vianey-Liaud et al., 1995 ; Schmidt-Kittler et al., 1997). Les autres éléments du complexe masticateur, la mandibule notamment, évoluent également en même temps que les changements de la morphologie dentaire. L'apophyse coronoïde s'abaisse entre I. medius et I. minor, pour demeurer très basse chez les autres espèces. Cette évolution s'accompagne d'un étirement postérieur de l'apophyse angulaire, ainsi que d'une horizontalisation de la crête massétérique inférieure qui devient prédominante à la mandibule à partir d'I. pauffiensis (e.g., Vianey-Liaud, 1976 : 35-46, figs.16-26). Cette morphologie mandibulaire, liée à une surface de trituration plane, va de pair avec l'allongement consécutif des masséters et ptérygoides. L'analyse des micro-usures dentaires permet de constater des mouvements masticatoires d'orientation qui varie d'oblique à propalinale à partir d'I. pauffiensis. La morphologie du complexe masticateur de ces Issiodoromys hypsodontes rappelle celle de certains Hystricognathi, tels que les Caviidae (Hautier et al., 2010), alors que les premiers Issiodoromys sont plus clairement sciurognathes.

L'ensemble de la morphologie crânienne est connu pour les seuls $I$. minor et $I$. pseudanaema. On constate un raccourcissement du palais entre $I$. minor (fpa au niveau du milieu de P4 et bord antérieur des choanes au milieu de M3) et I. pseudanaema (fpa à l'avant de M1 et bord antérieur des choanes au niveau de la partie postérieure de M2). Dans les deux cas, les bulles auditives sont très volumineuses et cloisonnées (cinq compartiments chez I. minor), dispositif que l'on met en relation avec la nécessité de communiquer pour les individus de la même espèce répartis sur un large territoire et avec celle d'échapper aux prédateurs. C'est notamment le cas des espèces actuelles de rongeurs vivant en habitats ouverts (e.g., Webster \& Webster, 1980 ; Kotler, 1985). De tels habitats seraient donc disponibles au moins à partir du Rupélien supérieur. Les caractéristiques du squelette postcrânien d'I. pseudanaema (MP29) indiquent un animal sauteur spécialisé et traduisent ainsi un même type d'habitat (Lavocat, 1951 ; Vianey-Liaud et al., 2015). Chez cette espèce, on dénombre trois orteils allongés et tibia et fibula sont fusionnés. Issiodoromys medius (MP22-23), dont les proportions des membres indiquent que l'animal était adapté au saut, est encore pourvu de cinq orteils et son tibia est distinct de la fibula. Il a été possible de mettre en évidence une évolution de l'appareil locomoteur entre $I$. medius, I. minor (MP24-MP25) et I. pseudanaema qui traduit une plus grande spécialisation au saut (Vianey-Liaud et al., 2015). S'il n'est pas possible de suivre les changements de la morphologie du pied entre I. medius et I. pseudanaema, par contre la fusion tibia-fibula intervient à partir d'I. pauffiensis (MP 26) alors que chez I. minor, la fibula est encore distincte du tibia.

Cette lignée était supposée s'enraciner dans les Elfomys éocènes, notamment $E$. hartenbergeri (Vianey-Liaud \& Ringeade, 1993). L'analyse des caractères que nous avons menée et la topologie en dichotomies successives des Issiodoromys sur AG, avec à sa base l'espèce hartenbergeri, soutient cette hypothèse et nous conduit à l'attribuer aussi au genre Issiodoromys. Issiodoromys nanus, contemporain de $I$. medius est intercalé entre cette dernière et $I$. hartenbergeri, ce qui souligne la morphologie conservatrice de la petite espèce d'Issiodoromys qui représente une deuxième lignée parallèle à la lignée principale. Elle est connue pour l'instant seulement en Espagne et elle est moins bien caractérisée que les autres taxons, car peu documentée.

Les relations de parenté montrent que l'ensemble Pseudoltinomys cuvieri + Pseudoltinomys oligocènes constitue un clade comprenant une dichotomie interne plutôt qu'un clade incluant des espèces arrangées de façon hiérarchique. $P$. cuvieri et $P$. gaillardi sont rapprochées : ce sont les espèces les plus anciennes, respectivement MP19-20 et MP21. Pseudoltinomys amblesi et $P$. major forment l'autre fraction du clade : ce sont les espèces les plus récentes, possiblement contemporaines (MP22-MP23) ; il s'agit des espèces les plus grandes du genre chez lesquelles les prémolaires inférieures notamment sont de taille légèrement supérieure à celle des molaires. Chez tous les autres Issiodoromyinae, les prémolaires sont de taille nettement plus petite. Cette lignée ne montre pas de transformations de la morphologie dentaire aussi poussées que celles des Issiodoromys et la lignée disparaît dès le Rupélien inférieur (MP22/23). Pseudoltinomys diffère des Issiodoromys par la branche montante de la mandibule plus haute (condyle articulaire et apophyse coronoïde hauts) et une forte orientation latéro-médiale des muscles masséter superficiel et ptérygoïde interne. L'orientation des micro-usures de la surface occlusale des dents jugales vient appuyer l'hypothèse d'une relation entre cette morphologie de la mandibule et des mouvements de mastication obliques (Hautier et al., 2010).

Les résultats de l'analyse AG (Fig. 7B) montrent que les Pseudoltinomys plus anciens s'organisent en une séquence de branchements en amont du précédent clade Pseudoltinomys. Il est à noter que ces branchements successifs suivent un ordre chronologique qui, dans une certaine mesure, pourrait être compatible avec les stades successifs d'une lignée 
évolutive. Cependant, certaines de ces espèces sont encore mal caractérisées et devraient être revues, notamment celles du niveau MP17 (P. phosphoricus, $P$. cosetanus et P. mamertensis). Par ailleurs, celles de la fin du Bartonien (MP15/16 et MP16) peuvent être discriminées des espèces contemporaines d'Elfomys sur des critères extrêmement pointus, montrant que ces deux lignées s'enracinent précocement dans l'Eocène moyen (Comte et al., 2012 : 215-228).

Dans notre analyse, les espèces du genre Elfomys constituent la première radiation des Issiodoromyinae, groupe-frère des deux autres genres, Issiodoromys et Pseudoltinomys. Ces espèces sont organisées là encore en branchements successifs sur la topologie obtenue, le premier rameau correspondant à l'espèce la plus ancienne montrant les caractères les plus primitifs (E. catalaunicus Bonilla-Salomón et al., 2016 ; MP15), les suivantes pouvant être considérées comme stades évolutifs plus avancés d'une même lignée.

\section{- Les lignées de Theridomyinae}

\section{Le clade et les lignées de Protechimys}

Jusqu'au milieu du $20^{\text {ème }}$ siècle, ce genre n'a été représenté que par trois espèces du début du Chattien ( $P$. gracilis, $P$. blainvillei et $P$. major). Depuis, des espèces plus anciennes ont intégré le genre (e.g., Vianey-liaud, 1998). L'hypothèse d'une lignée s'enracinant à la fin du Rupélien avec $P$. truci (MP23/24) a été avancée et argumentée, et des stades évolutifs transitionnels avec $P$. gracilis - P. blainvillei on été nommés.

Comme indiquéplus haut, 1 'arrangement successifdes espèces de Protechimys s'effectue dans un ordre inverse de l'ordre stratigraphique. Cette disposition est très vraisemblablement due au fort parallélisme qui affecte l'évolution des molaires inférieures chez les Archaeomyini. Aussi, la morphologie des molaires inférieures de l'espèce terminale de la lignée Protechimys ( $P$. major) est très convergente avec celle des molaires inférieures d'Archaeomys.

De $P$. truci à $P$. major, l'obliquité des crêtes augmente et l'inclinaison plus forte des couronnes entraîne celle des flexus et flexides (téniodontie). De ce fait, l'épaisseur de l'émail se différencie entre les flancs opposés des lophes, lophides, flexus, flexides, la différence entre les flancs s'accentuant. Le sinuside s'enfonce plus profondément vers le bord lingual, tandis que le sIII se réduit, pour disparaître seulement chez $P$. major. En même temps, les sI et sIII se réduisent : peu profonds, ils sont très éphémères, le sI plus que le sIV, ce dernier s'effaçant précocement sur quelques dents de $P$. blainvillei et sur la plupart chez $P$. major. La surface occlusale s'aplanit. La longueur des $\mathrm{p} 4 \mathrm{~s}$ et $\mathrm{dp} 4 \mathrm{~s}$ n'augmente pas par rapport à celle des molaires. Aux dents supérieures, les synclinaux buccaux (antérieurs S0-SI) diminuent, le SII également jusqu'à disparaître chez $P$. major, tandis que le sinus s'allonge jusqu'à s'ouvrir buccalement. A partir de $P$. gracilis et $P$. blainvillei, la mure des DP4s s'interrompt. Du cément dans le fond des flexus et flexides vient renforcer les faces amincies de l'émail seulement chez $P$. major. Les deux espèces $P$. gracilis et $P$. blainvillei qui coexistent autour de MP 25, témoignent de la différenciation de deux lignées parallèles. Ces deux espèces, outre les différences dans la morphologie dentaire (e.g., sII ouvert lingualement chez blainvillei et fermé chez gracilis; $\mathrm{cf}$. Vianey-Liaud et al., 1995), se distinguent dans la forme et les proportions du palais ou de la mandibule (Vianey-liaud, 1998 : 273). Disposant de plusieurs populations rapportées aux trois espèces de Protechimys issues de cinq gisements du Quercy qui s'échelonnent dans le temps à la transition Oligocène inférieur - Oligocène supérieur, l'analyse de leur variabilité a montré la différenciation progressive des morphotypes au sein des espèces successives des deux lignées de Protechimys $(P$. major et $P$. gracilis) à partir de $P$. gracilis (Vianey-Liaud \& Michaux, 2003 : fig. 7).

\section{Le clade et les lignées d'Archaeomys}

Pour ce genre, les résultats de notre analyse montrent à nouveau un arrangement hiérarchique (i.e., dichotomies successives) des espèces, lesquelles correspondent aux hypothèses de relations de parenté envisagées jusque-là. Les trois espèces dont l'attribution générique n'est pas encore stabilisée, désignées ici sous le nom de genre double Archaeomys/ Blainvillimys, s'inscrivent en branchements basaux du clade Archaeomys. L'origine de ces espèces, vraisemblablement à partir d'éléments de la lignée des Blainvillimys oligocènes, est encore en discussion (e.g., Vianey-Liaud, 2015). Trois lignées parallèles d'Archaeomys sont proposées à la suite de Mödden (1993), incluant les Archaeomys/Blainvillimys, mais la caractérisation et la distinction des espèces n'est pas encore claire (cf. Systématique, p. 46-48). De façon globale, l'hypsodontie et la téniodontie augmentent au cours du Chattien, de MP27 à MP30. La taille augmente également, au moins chez $A$. laurillardi, tandis qu'A. arvernensis est plus petite. L'antésinus des DP4s s'allonge transversalement et un synclinal postérieur additionnel se développe (SV).

Toutes les espèces, du moins celles qui sont suffisamment documentées, montrent un palais triangulaire (plus étroit à l'avant qu'à l'arrière). Il est long car le foramen palatin antérieur se termine bien avant DP4 ou P4, au niveau du bord antérieur de l'arc zygomatique. Cet allongement antérieur du palais semble aller de pair avec l'augmentation importante de la taille des DP4s et P4s. L'incisive raccourcit entre $A$. intermedius et $A$. laurillardi.

\section{Les clades et les lignées de Blainvillimys}

La discussion sur le périmètre du genre Blainvillimys a montré les diverses questions qui demeurent, notamment pour les formes éocènes (cf. p. 43-45). Comme il n'est pas dans notre propos d'y apporter toutes les réponses nécessaires, il ne sera question ici que des éléments d'une lignée jusqu'ici bien établie, même si les caractéristiques de certains de ses stades demandent à être complétées.

L'espèce-type du genre (B. blainvillei), datée de l'Oligocène supérieur, montre les caractéristiques parmi les plus dérivées du genre, dans la lignée « $B$. » langei - B. gregarius - helmeri $(=$ ?varians $)$ - blainvillei - stehlini. Elle est caractérisée par l'accroissement de la hauteur de la couronne, la généralisation $\mathrm{du}$ sI, mais celui-ci reste très peu profond et, du fait de l'hypsodontie, disparaît de plus en plus vite avec l'usure. S'ajoutant à l'augmentation de hauteur, le fût de la couronne et le sinuside notamment s'inclinent mésio-distalement aux dents inférieures, légèrement obliquement de mésio-lingual à distobuccal aux dents supérieures. Ceci influe sur l'orientation des lophes et lophides ainsi que sur la forme des sinus et sinusides. L'enfoncement transverse de ces derniers et le raccourcissement concommittant des synclinaux et synclinides, participent à la modification des surfaces d'usure. Enfin, l'allongement de plus en plus important des dp4s et DP4s par rapport aux molaires est aussi une caractéristique des Blainvillimys 
oligocènes par rapport aux formes éocènes, cette tendance étant particulièrement est bien marquée dans cette lignée.

Blainvillimys heimersheimensis (MP24) constitue un rameau divergent peut-être relié à $B$. gemellus (MP22), mais les éléments manquent actuellement pour mieux argumenter cette autre possible lignée.

\section{LES RADIATIONS ADAPTATIVES ET LES EXTINCTIONS}

\section{Description et relations avec les changements paléoenvi- ronnementaux physiques et biologiques}

Les espèces de théridomorphes sont repérées dans les différents niveaux mammaliens MP. Nous reviendrons plus loin sur la signification et l'étalonnage de ces MP : il suffit de noter pour l'instant qu'ils permettent de situer ces espèces dans la GTS et donc par rapport aux divers événements climatiques qui lui sont corrélés. Le nombre d'espèces permet d'estimer la diversité du groupe à un niveau donné, des changements étant observés tout au long de son histoire (Fig. 3).

Une première radiation est enregistrée à la fin de l'Eocène inférieur avec la différenciation des Theridomorpha au sein des Microparamyinae (Pantrogna et Hartenbergeromys), au moment où les températures amorcent une baisse après les épisodes chauds de l'Yprésien inférieur. Cette baisse se poursuit pendant tout le Lutétien, jusqu'au Bartonien basal, moment où survient un épisode bref et intense de réchauffement (MECO, pour « Middle Eocene Climatic Optimum »; e.g., Cramer et al., 2009).

Une deuxième radiation, au cours du Lutétien, est à l'origine du genre Protadelomys, qui témoigne des premiers Theridomyoidea, documentés dans la partie méridionale de leur territoire d'Europe Occidentale. Ce genre est enregistré jusqu'à la base du Bartonien, période pendant laquelle se produit une troisième radiation, qui est à l'origine de la dichotomie Pseudosciuridae - Theridomyidae, avec Tardenomys comme groupe souche de l'ensemble. Elle pourrait être liée au bref et intense événement du MECO. Ceci devra cependant être confirmé par des études visant à reconstituer les paramètres climatiques en milieu continental de la fin de l'Yprésien au Bartonien basal.

Les épisodes de diversification, au sein des Pseudociuridae et des Theridomyidae se sont enchainés au Bartonien supérieur et au Priabonien, tandis que plusieurs oscillations importantes des températures sont enregistrées en milieu marin, modulant la tendance à une baisse générale modérée des températures. Ainsi un réchauffement est noté vers $39 \mathrm{Ma}$, suivi par un net refroidissement entre 39 et $38 \mathrm{Ma}$. Les températures demeurent ensuite relativement élevées jusqu'à $37 \mathrm{Ma}$, pour retrouver le niveau connu avant $38 \mathrm{Ma}\left(\delta_{18} \mathrm{O}=1,0 \%\right)$ et le restent jusque vers $34 \mathrm{Ma}$ (e.g., Cramer et al. 2009).

Nos connaissances sur la végétation en Europe occidentale pour cette période montrent qu'elle a enregistré les fluctuations reconnues en milieu marin mais différemment selon la latitude, l'altitude et la proximité des terres avec les étendues marines, Téthys ou Atlantique (e.g., Collinson \& Hooker, 2003). Le timing des changements de la végétation depuis les végétations subtropicales du début de l'Eocène varie en conséquence suivant les aires géographiques. Collinson \& Hooker (2003) considèrent que la végétation de l'Eocène supérieur et de l'Oligocène en Europe Occidentale combinait des essences à feuilles caduques et à feuilles persistantes, avec des proportions variables suivant les époques et les lieux; le climat était globalement chaud et humide, les hivers étant légèrement plus arides que les étés. Au cours de l'Oligocène, la partie la plus méridionale de l'aire de répartition des Theridomyoidea (Espagne, sud de la France) devait connaître un climat plus aride, comme en témoigne la proportion relativement importante de xérophytes (Collinson \& Hooker, 2003).

Si au Bartonien terminal une seule lignée est très hypsodonte (Bernardia), le Bartonien supérieur voit se différencier plusieurs radiations qui incluent des formes hypsodontes (Patriotheridomyinae, Oltinomyinae, Theridomyinae et Issiodoromys) présentes sur l'ensemble de leur territoire mais aussi des formes sélénodontes (Pseudosciurinae) surtout dans la partie la plus septentrionale de leur territoire (cf. Fig. 2). Les rongeurs actuels hypsodontes et sélénodontes apportent des indications précieuses qui permettent d'avancer une différenciation des theridomyidae hypsodontes en relation avec la présence de milieux ouverts, a minima de vastes clairières dans les environnements forestiers tandis que les formes sélénodontes sont le plus souvent liées à des habitats forestiers (e.g., Andó et al., 1985). Un certain provincialisme s'exprime dans la répartition des différentes sous-familles ou genres. Les Issiodoromyinae sont absents d'Allemagne du sud (Jura Souabe) pendant tout l'Eocène supérieur, tandis que les Pseudosciurinae s'y différencient.

Pendant toute l'existence éocène des Theridomyoidea, des disparitions d'espèces se sont produites mais rares sont les extinctions «vraies ». La plupart correspondent en effet à des pseudo-extinctions, des espèces apparaissant par évolution à partir d'espèces préexistantes (cf. ci-dessus, lignées). On peut faire la part des unes et des autres dans certains cas (Patriotheridomys, Elfomys, Issiodoromys, Thalerimys, Pseudoltinomys pro parte, Blainvillimys pro parte et Theridomys pro parte) mais pour les autres cas, s'agissant d'espèces mal documentées ou peu étudiées, des analyses complémentaires restent à faire.

La transition Eocène/Oligocène (33,9 Ma) est marquée par la disparition simultanée d'une partie des formes hypsodontes (Patriotheridomyinae, Oltinomyinae, Thalerimys et quelques autres lignées de Theridomyinae) et au moins de Treposciurus chez les brachyodontes. Ce pic d'extinctions est contemporain de la première phase glaciaire de l'Oligocène (Oil ; e.g., Cramer et al., 2009) et participe à l'événement faunique de la "Grande Coupure », que caractérise l'arrivée massive en Europe occidentale de migrants asiatiques. Les effets du refroidissement global ont été cependant atténués par la proximité d'une mer chaude, la Tethys et du relatif éloignement de l'Europe sud-ouest Occidentale de l'Océan Atlantique (e.g., Kocsis et al., 2014).

Entre la fin de l'Eocène et le début de l'Oligocène plusieurs évènements tectoniques ont modifié la géographie de l'Europe du sud-ouest. La chaîne pyrénéenne qui poursuit sa surrection permet le développement des grands bassins sédimentaires intracontinentaux entre elle et les cordillères catalanes (notamment Bassin de l'Ebre) ; au moins jusqu'à la transition Oligo-Miocène, avant la rotation du bloc Corso-Sarde, la Méditerranée n'est pas encore formée dans sa partie nordoccidentale (e.g., Schettino \& Turco, 2011). Au nord-est de l'Europe Occidentale, les changements sont plus importants. Du fait de l'action conjuguée de la baisse du niveau des océans et de la tectonique, le bras de mer qui reliait la Paratéthys à l'Océan Arctique se ferme au niveau du détroit de Turgaï au sud de l'Oural actuel. Ceci a permis une communication 
terrestre entre domaines asiatiques et européens. Sur la partie méridionale de l'Eurasie, l'orogenèse alpine (Alpes, Dinarides et Carpathes) a entraîné la formation d'une barrière entre les domaines de la Téthys et de la Paratéthys et favorisé des passages terrestres occasionnels d'est en ouest. De ce fait des routes ont été ouvertes essentiellement pour les faunes est-européennes et asiatiques par deux voies possibles, l'une plus septentrionale et relativement continue, l'autre, plus méridionale et discontinue, au long des îles et archipels occupant la région des Balkans.

Les parts respectives de la forte variation environnementale et de la compétition avec les immigrants dans la disparition de beaucoup de Theridomyoidea au moment de la «Grande Coupure » restent difficiles à établir. On ne peut que noter l'étroite corrélation temporelle de tous ces événements. Les Theridomyoidea entrent en contact avec au moins cinq familles de rongeurs (Cricetidae, Aplodontidae, Eomyidae, Sciuridae, Castoridae) jusque-là absents du territoire européen (e.g., López \& Thaler, 1975), tous brachyodontes hormis les Castoridae, ainsi qu'avec des Lagomorpha. Au moins deux espèces de lagomorphes atteignent l'Europe Occidentale, du côté du Jura Souabe d'une part et du sud de la France (Quercy) d'autre part (Lopez \& Thaler, 1975 ; Heissig \& Schmidt-Kittler, 1972 ; Vianey-liaud \& Lebrun, 2013), empruntant peut-être chacune une des deux voies de communication. Ces migrations n'ont cependant pas eu de succès, puisqu'on ne retrouve pas d'autres lagomorphes avant l'Oligocène supérieur.

Par ailleurs, la contemporanéité de l'arrivée des migrants a été discutée à plusieurs reprises du fait de la datation relative problématique de deux localités du Jura Souabe, Möhren 13 (MP21 ou MP22) et Möhren 19 (MP20 ou MP21) (SchmidtKittler \& Vianey-Liaud, 1975 ; Sigé \& Vianey-Liaud, 1979 ; Legendre, 1987). Ces deux localités qui ont livré l'émigrant Atavocricetodon atavus, démontreraient l'arrivée plus précoce en Allemagne du sud des Cricetidae, si Möhren 19 était bien rapprochée du niveau MP20 de l'Eocène supérieur. La révison des Theridomys et des Suevosciurus de ces localités (Gad, 1987 ; Heissig, 1987) lève alors l'ambiguïté en établissant la contemporanéité des migrations, en corrélant Möhren 19 avec MP21 (et Möhren 13 avec MP22).

Les Theridomyinae se diversifient à nouveau après l'épisode d'extinction de la « Grande Coupure » et les Columbomyinae, dont l'origine est commune avec les Patriotheridomyinae, apparaissent(Figs. 7B et 21). La suite des radiations adaptives des Theridomyoidea a pris place dans un environnement fortement changeant, et peut-être notamment en raison des nombreux épisodes glaciaires maintenant répertoriés durant le Rupélien. En effet, au moins cinq épisodes sont distingués (Zachos et al., 2008 ; Cramer et al., $2009: \delta_{18} \mathrm{O}$ compris entre 2,5 et 1,5\%o), jalonnant une baisse globale des températures. Un changement drastique dans la végétation au niveau des moyennes et hautes latitudes de l'hémisphère nord a été fortement argumenté, notamment d'après l'étude des flores nord-américaines (TEE = «Terminal Eocene Event » ; e.g., Wolfe, 1978 ; Hubbard \& Boulter, 1983). Cependant, des changements de végétation plus progressifs ont été envisagés en Europe (e.g., Collinson $\&$ Hooker, 2003). En tous cas, les variations saisonnières sont devenues plus contrastées, un changement qui a mis fin aux forêts tropicales, telles qu'observées dans l'Eocène moyen, au profit de forêts mésophiles.

Au cours du Rupélien et jusqu'au Rupélien terminal, des incursions marines limitées et épisodiques ont eu lieu à la faveur d'épisodes de « rifting ». Elles ont laissé des témoins dans le fossé rhénan communiquant épisodiquement avec le bassin de la molasse Suisse (Picot et al., 2008), dans le fossé des Limagnes, ou bien dans la vaste zone en distension du bassin de Marseille (cf. Nury, 1988). De vastes étendues d'eaux saumâtres à salées se sont évaporées ; en partie du fait de l'aridité du climat qui s'est maintenue tout au long de la période, des dépôts de gypses, potasse et sulfates en ont résulté dans les zones à forte subsidence comme en Alsace. Le fossé de Limagne a fonctionné plus généralement comme un bassin endorhéique où d'épais stromatolites se sont développés. La surrection de l'arc des Alpes et Dinarides a provoqué une élévation de cette zone, à 1200 m environ (Kócsis et al., 2014), ce qui a contribué aux différenciations climatiques depuis le sud-ouest de la zone (Espagne) jusqu'au nord-est (Jura suisse et Souabe).

Ces variations paléogéographiques, combinées avec l'existence d'une zonation paléoclimatique vraisemblable, ont de toute évidence été les facteurs de différenciation dans la répartition des rongeurs et plus particulièrement des différents théridomyoïdes. La lignée des Pseudosciurus, sélénodonte et inféodée propablement à un couvert forestier, a évolué seulement au sud de l'Allemagne (MP18 à MP22). A l'inverse, les Issiodoromyinae ne sont arrivés en Allemagne qu'à partir de la fin du Rupélien/base du Chattien (MP24) dans le bassin de Mainz, puis dans le Jura Souabe à partir du Chattien supérieur (MP28-30). Les Columbomyinae ont essentiellement colonisé la zone ibéro-franco-suisse ; parmi eux, les Columbomys sont restés dans l'aire la plus méridonale.

$\mathrm{Au}$ Chattien inférieur, deux épisodes de glaciation plus intenses sont recensés (Cramer et al., 2009 : Oi 2a et 2b). A ce moment-là, tous les Pseudosciuridae ont déjà disparu. La dernière et de moindre importance radiation des Theridomyinae hypsodontes, celle des Archaeomys, se produit ensuite. Les transformations morphologiques qui s'opèrent dans trois grandes lignées, Archaeomys/Blainvillimys, Columbomys et Issiodoromys, s'accélèrent à partir du début du Chattien (entre MP25 et MP26) et notamment l'hypsodontie augmente fortement.

Un réchauffement important est enregistré à partir de 26,5 Ma environ, ramenant des conditions subtropicales et humides (LOWE = «Late Oligocene Warming Event »; cf. Zachos et al., 2001 ; de Man \& van Simaeys, 2004). D'autres groupes de rongeurs, Gliridae et Eomyidae notamment, voient leur diversité s'accroître sensiblement dans ces conditions où les espaces couverts reprennent de l'importance (Comte \& Vianey-Liaud, 1986), et d'autres genres de lagomorphes sont alors répertoriés (e.g., Tobien, 1963 ; Hugueney, 1969 ; Mörs \& Kalthoff, 2010). L'étude de Poschmann et al. (2010) sur la reconstitution des paramètres climatiques ayant présidé à la formation du gisement d'Enspel (Westerwald, Allemagne ; MP28, 24,7 Ma) a permis d'estimer la température moyenne annuelle autour de $15-17^{\circ} \mathrm{C}$, avec une température maximale autour de $25^{\circ} \mathrm{C}$ et minimale autour de $5-7^{\circ} \mathrm{C}$, la moyenne des précipitations annuelles ayant été estimée autour de $900 \mathrm{~mm}$ (mais avec des pics allant jusqu'à $1355 \mathrm{~mm}$ ). Ces températures sont légèrement inférieures à celles estimées pour la flore plus récente de Rott-Thierbach (MP30).

Dans ce contexte, la forte spécialisation des lignées subsistantes n'apparaît pas favorable, dans un contexte de compétition renouvelé et un environnement moins ouvert que celui qui a accompagné leur succès évolutif. De plus, la faible diversité des Theridomyoidea subsistants n'est pas suffisante pour assurer une nouvelle radiation adaptative et la persistance du groupe, comme ce fut le cas lors de la « Grande Coupure ». Le dernier Columbomys, au sud, disparaît avant la fin de l'Oligocène (MP29) et les quelques espèces d'Archaeomys et 
d'Issiodoromys à peine quelques temps après (MP30).

La répartition paléogéographique des différents genres de Theridomyidae durant le Paléogène s'est faite sur le territoire ouest européen, depuis le nord de la péninsule ibérique jusqu'au $50^{\circ}$ parallèle au nord, sans dépasser la République Tchèque à l'est. Elle a été soumise aux variations des contraintes environnementales (température, humidité et végétation). Ces contraintes ont joué un rôle majeur sur les transformations sélectionnées de plusieurs lignées au cours du temps, notamment les hypsodontes. L'existence ou la levée de barrières géographiques, en complément des autres facteurs, a contribué aux variations de leurs distributions géographiques, mais ont aussi conditionné leurs diversifications. La levée de ces obstacles a changé la répartition du groupe. En outre, l'arrivée des espèces immigrantes en Europe Occidentale, espèces susceptibles d'avoir été concurrentes, a très certainement contribué aux diverses phases d'extinction et notamment celles de la «Grande Coupure » et aussi toutes celles qui ont ponctué leur histoire au cours de l'Oligocène.

\section{Les conséquences des radiations successives}

\section{Sur la diversité du groupe (Fig. 3)}

La diversité des Theridomyoidea (diversifications cumulées et extinctions) montre deux pics au cours de l'Eocène supérieur, le premier au Bartonien (MP16) autour de 39 Ma et le deuxième, plus important, vers $34,8 \mathrm{Ma}$ (MP19). Le premier concerne majoritairement des formes brachyodontes, le second est principalement généré par les radiations de formes hyspodontes, Patriotheridomys, Pseudoltinomys et Theridomyinae.

La « Grande Coupure » marque une chute de la moitié de la diversité, cette diminution affectant à la fois les formes brachyodontes et hypsodontes. La diversité globale s'est toutefois maintenue pendant les trois premiers millions d'années du Rupélien, du fait essentiellement de la diversification des Theridomyidae, les Pseudosciuridae ayant fortement décliné. Ces derniers disparaissent à la fin du Rupélien, tandis qu'une dernière radiation adaptative de faible intensité se produit chez les Archaeomyini, avant l'extinction du groupe à la fin de l'Oligocène.

\section{Les parallélismes et convergences}

Les radiations successives et les transformations morphologiques associées qui se produisent dans les lignées ont multiplié les cas d'évolutions parallèles que seules des analyses morphologiques ou morphométriques approfondies peuvent déceler. S'ajoutent également des convergences des patrons dentaires entre taxons plus éloignés, au sein des Theridomyoidea mais aussi avec des taxons extérieurs à cette famille. Les évolutions convergentes de la morphologie dentaire sont connues de longue date chez les rongeurs (e.g., Wood, 1937 ; Butler, 1985). Elles traduisent la réponse à de fortes contraintes adaptatives semblables.

\section{- Parallélismes au sein des Theridomyinae}

Quatre genres, Blainvillimys, Toniodus et Protechimys, à la fin de l'Oligocène inférieur et Archaeomys, au cours de l'Oligocène supérieur, montrent les mêmes tendances évolutives. Ils sont caractérisés par une inclinaison antéro-postérieure des dents inférieures et oblique mésiolinguo-buccodistale des dents supérieures, plus ou moins prononcée, allant de pair avec une augmentation asymétrique de la hauteur des couronnes. Cette inclinaison s'accompagne aussi d'un fort amincissement des lamelles d'émail sur le bord de fuite des lophes et des lophides (e.g., Vianey-Liaud \& Schmid, 2009). Au moins au cours du Rupélien, les différences morphologiques entre chacun des types sont relativement claires (cf. chapitres Systématique et Lignées).

Toutes les molaires inférieures de Toniodus curvistriatus sont pourvues d'un lophide postérieur (= hypoconide + postérolophide) isolé des autres crêtes. Protechimys montre des molaires inférieures séparées en deux par une profonde rupture médiane et le synclinide IV existe jusqu'à MP25 (P. gracilis et $P$. blainvillei). Chez Archaeomys, cette profonde rupture est aussi la règle, mais s'y ajoute la perte de la vallée postérieure (synclinide IV). Or, à partir de MP26, Protechimys major, ainsi que les espèces pour l'instant rangées dans Archaeomys/ Blainvillimys (MP26 et MP27) et tous les Archaeomys (MP28-30) montrent la même structure générale des molaires inférieures, sans sIV. Si on considère les dents supérieures de ces espèces, on ajoute Blainvillimys blainvillei et B. stehlini dans le lot des espèces affectées d'un fort parallélisme, avec l'allongement du sinus et la réduction des synclinaux externes. Mais ces deux espèces ont aussi des molaires inférieures avec une rupture médiane presque complète, l'ectolophide étant proche du bord interne, avec un sIII fortement réduit. C'est sur les détails de la morphologie des petits synclinaux externes des dents supérieures que Mödden (1993) s'est fondé pour tenter de définir des critères de distinction entre les espèces rupéliennes et chattiennes de ces genres et identifier des relations de parenté éventuelles (cf. Systématique et Lignées).

Les trois genres Pseudoltinomys, Elfomys et Issiodoromys montrent un schéma dentaire très proche et parfois, peu de différences de taille (cf. Systématique et Lignées). A partir de la base du Priabonien pour Pseudoltinomys et au Priabonien supérieur pour Issiodoromys, on observe parallèlement un accroissement de la semi-hypsodontie. Ultérieurement, à partir du début de l'Oligocène, l'émail des incisives, qui était paucisérié $\mathrm{du}$ moins chez deux espèces éocènes d'Issiodoromyinae, (Elfomys sp. et Pseudoltinomys phosphoricus), devient parallèlement unisérié chez Pseudoltinomys gaillardi d'une part et chez tous les Issidodoromys d'autre part (Martin, 1999). Si les patrons des molaires isolées des deux genres se ressemblent beaucoup, des différences apparaissent lorsque l'on considère aussi les prémolaires déciduales et permanentes. Leurs degrés d'allongement, tout comme la rapidité d'accroissement de l'hypsodontie, évoluent différemment dans les deux genres. On connaît peu ou pas les éléments de la morphologie crânienne et mandibulaire des derniers Pseudoltinomys ( $P$. major et $P$. amblesi). Par contre, les mandibules (et leurs dents) des deux genres au cours du Rupélien inférieur montrent une évolution distincte de leur mécanique masticatrice.

\section{- Parallélismes au sein des Theridomyidae}

Plusieurs lignées semi-hypsodontes (Theridomys, Blainvillimys, Thalerimys et Patriotheridomys) ont acquis un sI aux molaires inférieures. Stehlin \& Schaub (1951) insistaient sur la singularité des molaires inférieures de Theridomys (Trechomys) bonduelli du fait de la présence du sI aux dents inférieures (qu'ils supposaient liée à l'existence d'un paraconide, caractère primitif). Depuis, il est clairement apparu qu'il ne s'agissait pas d'un paraconide mais d'une néoformation au niveau de l'antérolophide et/ou du métalophide I, qui se développe à plusieurs reprises dans différentes lignées. Cette acquisition 
se produit de façon parallèle, diachrone ou isochrone, depuis le Priabonien jusqu'au Rupélien (e.g., Thaler, 1969 ; VianeyLiaud, 1972a, 1989). Elle peut affecter également tous les loci ou seulement certains, ou être variable (variation individuelle) au sein des espèces (cf. Lignées, p. 51). Ce caractère s'ajoute aux autres caractéristiques des genres que l'on utilise pour les discriminer (proportions des différents loci, existence ou non d'antésinuside, mure/endolophe présents ou absents aux dents supérieures, etc...).

Les prémolaires déciduales sont fortement allongées chez tous les Theridomyinae, chez Patriotheridomys et Issiodoromys (Figs. 5 et 6). L'analyse morphologique fine dans chaque genre a permis de discriminer les différentes structures impliquées dans cet allongement. Le repérage des homologies a été rendu possible par le suivi des espèces au cours du temps et la connaissance de tous les loci dentaires pour une espèce donnée, notamment pour les dp4s inférieures. Pour les DP4s supérieures des Theridomyinae, l'allongement débute par l'apparition d'une crête additionnelle subdivisant le SI (en S0 + SI) dès le Priabonien, et ce de manière parallèle au sein des genres Blainvillimys, Theridomys, Thalerimys et de l'espèce Patriotheridomys altus (MP19-20). Cette crête n'est pas développée chez Patriotheridomys neboulensis (MP18b) et $P$. sudrei (MP18a). Quelques DP4s d'Archaeomys huerzeleri (MP26-27) et Archaeomys quercyi (MP28a) montrent parfois un cinquième synclinal; il est plus fréquent chez $A$. intermedius (MP28b) (Fig. 18) et constant chez les autres Archaeomys (MP29-30). Le SV est rarement présent sur les molaires d'Archaeomys quercyi et fréquemment sur celles des autres Archaeomys (Fig. 16).

La microstucture de l'émail des incisives est unisériée dans deux clades, celui des Issiodoromyinae oligocènes et celui des Patriotheridomyinae. Dans le clade des Blainvillimys oligocènes, l'émail des incisives évolue de paucisérié-incliné à « pseudo-multisérié » (sensu Martin, 1999), tout comme dans le clade Protechimys-Archaeomys.

\section{- Convergences}

L'une des apomorphies des Theridomyoidea se rencontre aussi chez les Remyoidea. Il s'agit de la longue et oblique postprotocristide. Ce caractère a été un argument pour postuler l'hypothèse de proche parenté entre les deux taxons (e.g., Vianey-liaud et al., 1994).

Le plan pentalophodonte et l'hystricomorphie des Theridomyidae avait été considérés comme le signe d'une parenté possible entre les théridomorphes européens, les caviomorphes sud-américains ainsi qu'avec des rongeurs africains phiomyidés ou anomaluridés (jusqu'à Stehlin \& Schaub, 1951). La convergence dans l'acquisition de ces deux caractères a été depuis identifiée (e.g., Lavocat, 1955 ; Marivaux et al., 2002, 2004, 2011, 2014, 2015) et l'homologie du métalophe par exemple, est sujette à de nombreuses discussions (e.g., Hoffstetter \& Lavocat, 1970 ; Patterson \& Wood, 1982 ; Jaeger, 1989 ; Marivaux et al., 2002, 2004).

L'évolution de la morphologie du système masticateur des Issiodoromys (forme de la mandibule, du palais, orientation des rangées dentaires, mouvements propalinaux...) révèle un cas de convergence de forme avec certains Hystricognathi Caviidae, traduisant la prégnance des fortes contraintes adaptatives (Hautier et al., 2010, 2015).

\section{APPLICATIONS BIOCHRONOLOGIQUES}

\author{
Les niveaux-repères MP (Mammal Paleogene standard- \\ levels)
}

\section{Problématique}

Une échelle de niveaux repères mammaliens a été proposée pour matérialiser la succession temporelle des faunes mammaliennes paléogènes en Europe Occidentale. Le concept qui sous-tend l'usage des MP mérite d'être rappelé, car ces niveaux-repères à la source de l'échelle MP ont été parfois (voire souvent) utilisés comme des « biozones », ce qu'ils ne sont pas. Il suffit de citer une communication de Jaeger \& Hartenberger (1975) pour l'énoncer sobrement :

"Le niveau-repère (NR) possède des qualités intrinsèques particulièrement intéressantes :

- il constitue un instantané de l'histoire des faunes ;

- aucun problème de limite ne se pose, par définition ;

- les NR peuvent être multipliés au fur et à mesure du progrès des connaissances sans installer cette connaissance dans un système dogmatique, comme c'est le cas avec les zones ;

- le NR évite l'utilisation des associations d'espèces, trop souvent mises en contradiction par les faits ;

\section{Il doit être clairement précisé :}

- ...que la distinction entre deux NR successifs ne peut être établie que par référence aux degrés évolutifs différents observés au sein de lignées évolutives données ;

-... que le découpage en un certain nombre de régions biogéographiques en fonction des données géologiques constatées pour des périodes déterminées, doit précéder l'établissement d'une échelle valable pour chacune de ces régions.

Les problèmes de corrélations, d'une part entre provinces biogéographiques, d'autre part avec les étages marins, seront abordés par l'utilisation prioritaire des données de chronologie absolue et de paléomagnétisme...».

Chaque niveau-repère représente un instantané à l'échelle géologique, matérialisé par une faune repère, autrement dit, chaque échelon (MP) représente une ligne-temps. Chaque faune-repère est caractérisée par le niveau évolutif des espèces qui la composent. C'est à ces niveaux évolutifs que sont comparés les assemblages d'espèces de nouvelles localités. Ces assemblages peuvent être exactement similaires, alors la nouvelle localité est corrélée avec le même échelon MP. Si des différences sont constatées, avec un niveau évolutif plus avancé, ou moins avancé, sans atteindre celui du MP suivant, ou précédent, elles sont seulement rapprochées du MP, ou placées en position intermédiaire. Le cas échéant, des niveaux supplémentaires pourront être intercalés pour de nouveaux assemblages montrant des niveaux évolutifs bien définis et différents de ceux des MP existants.

L'intérêt de définir une échelle de niveaux-repères (« standard-levels ») exprimant la succession des stades évolutifs de faunes mammaliennes du Paléogène a émergé progressivement (e.g., Franzen, 1968 ; Hartenberger, 1969). Une première proposition d'échelle de niveaux-repères a été faite pour le Paléogène européen, avec une succession de faunes-repères, les MP n'étant pas encore formalisés (Russell et al., 1982). La définition des MP aura lieu lors du symposium sur la « Biostratigraphie et Paléoécologie Mammalienne du 
Paléogène Européen »(Schmidt-Kittler, 1987). La préface de Schmidt-Kittler, éditeur des actes de ce symposium, développe le concept (p. 15-16). Cette "philosophie » a eu du mal à être acceptée par l'ensemble des acteurs de la paléontologie mammalienne européenne, formés souvent à la biostratigraphie classique, aux biozones ou influencés par les étages mammaliens nord-américains. Un autre symposium a réuni ensuite les chercheurs européens travaillant sur les faunes mammaliennes paléogènes et néogènes. Ces derniers étaient porteurs de la biozonation qu'ils avaient établies pour le Néogène et ont tenu à continuer dans cette voie (Aguilar et al., BiochroM', 1997). Les obstacles rencontrés à la généralisation des NR étaient d'autant plus forts que les faunes mammaliennes étaient mal connues, notamment dans l'Eocène inférieur et moyen, ou bien que les contextes sédimentaire et stratigraphique étaient plus « stratigraphiquement corrects », comme pour le Néogène. Cette dernière situation concerne notamment le cas des formations sédimentaires épaisses dans lesquelles des gisements sont superposés (comme en Espagne, pour le Néogène et dans une moindre mesure, pour le Paléogène), ce qui donne l'illusion de pouvoir découper facilement le temps en tranches ayant une durée finie, mais ne s'affranchit aucunement du problème des limites. Les corrélations entre les bassins ne sont pas plus évidentes, du fait des multiples variations latérales de faciès et des nombreux arrêts de sédimentation liés notamment aux phases d'émersion, de pédogenèse ou d'érosion. Par ailleurs, les zones sont des tranches de temps épaisses relativement aux horizons-repères (niveaux-repères) et ne permettent pas la précision nécessaire pour analyser les changements évolutifs. Elles forment aussi un cadre rigide, beaucoup moins susceptible d'évoluer avec les progrès des connaissances.

\section{Calibration et évolution de l'échelle des niveaux-repères MP concernés par l'évolution des Theridomyoidea}

Au moment où les MP ont été définis, les points de calibration de l'échelle des MP étaient très rares, voire absents sur de longues tranches de temps. De ce fait, la première échelle a été constituée de niveaux équidistants, dans l'attente d'un étalonnage.

Les faunes mammaliennes européennes du Lutétien sont peu nombreuses, notamment celles ayant livré des Theridomyidae. Les localités types des trois NR (MP11-12-13) sont basées sur les séries continentales du Geiseltalt (Allemagne orientale) et aussi sur le gisement de Messel pour MP11 (Franzen, 2005 ; Merz \& Renne, 2005). Ce dernier a été récemment corrélé avec la fin de l'Yprésien final, daté autour de $48 \mathrm{Ma}$ (Lenz et al., 2015). Les assemblages fauniques mammaliens du Geiseltalt (UK, uMK et oMK) se différencient dans leur composition, mais on n'y distingue pas ou peu (seulement pour un Palaeotheriidae) de stades évolutifs de lignées différentes, du moins entre les deux derniers ; ils ne renferment aucun Theridomyoidea. La succession des trois NR du Lutétien est argumentée par les stades évolutifs des mammifères et les événements fauniques, les Paleotheriidae servant d'éléments de corrélation avec la série-type. Les Theridomyidae (Protadelomys) proviennent de localités corrélées de cette façon, donc indirectement, avec les NR du Lutétien. Ainsi, Peláez-Campomanes (1995) place Casa Ramón avec Protadelomys nievesae (Huesca, Espagne) en regard de MP11 ou 12. C'est sur la base de l'analyse des niveaux évolutifs des espèces de Protadelomys qu'Escarguel (e.g., 1999) distingue des niveaux intermédiaires entre MP13 et MP14 pour plusieurs localités de France et du Jura Suisse. Compte tenu de l'absence d'éléments de corrélation géochronologique et des questions concernant la mise en évidence de lignées évolutives, la robustesse de la définition des MP 12 à 14 doit être renforcée. Ces niveaux ne montrent pas de point de calibration, ni direct ni indirect; de ce fait leur position relative à la GTS est hypothétique. MP14 a été placé à la limite Lutétien/Bartonien, sur la base des degrés évolutifs de la macrofaune (essentiellement Perissodactyla et Artiodactyla ; Biochro'M97). Compte tenu de l'absence de calibration, ces niveaux sont équidistants sur la figure 2.

Le Bartonien (durée d'environ $3 \mathrm{Ma}$ ) comprenait trois NR (MP15 à 17). La faune repère de MP15 (La Livinière II) doit être abandonnée, car elle représente un assemblage de rongeurs très vraisemblablement mélangé et pauvre de surcroît (cf. Comte et al., 2012). Plusieurs faunes montrent des assemblages de stades évolutifs intermédiaires entre MP14 et MP16 : Sant-Jaume-deFrontyaná 1 (Bassin de l'Ebre, Espagne ; Bonilla-Salomón et.al., 2016), Chéry-Chartreuve (Aisne, France ; Comte et al., 2012) et les Alleveys (Jura Suisse ; Hooker \& Weidmann, 2007). Vu sa position dans une série stratigraphique fournissant plusieurs sites fossilifères superposés, Frontyaná 1 pourrait être candidat comme localité-type de MP15, Chéry-Chartreuve et Les-Alleveys se trouvant en position intermédiaire entre MP15 et MP16.

Quelques études magnétostratigraphiques ont été réalisées sur les séries du bassin de l'Ebre oriental, incluant le site fossilifère de Pontils 26 (Costa et al., 2013). Les résultats concernant cette localité ont été discutés par Comte et al. (2012) : elle ne peut pas représenter MP15, car elle est corrélée au chron 17r. Robiac (France), localité-type de MP16, est aussi corrélé à la base de ce chron (e.g., Hooker \& Weidmann, 2007 ; Comte et al., 2012), du fait des stades évolutifs partagés entre sa faune mammalienne, celle du Jura Suisse et celle des « Barton beds » d'Angleterre.

Plusieurs niveaux MP successifs ont été proposés dans le Priabonien sur la base de la succession des faunes mammaliennes (six pour une durée d'environ 3,7 Ma). Le niveau MP17 a été subdivisé en deux, MP17a, Fons 4 et MP17b, Perrière (Aguilar et al., BiochroM'97, 1997 : 787-788). Legendre \& Lévêque (1997) ont proposé de situer MP17a entre le chron C17n et la base (inverse) du chron C16, soit à la fin du Bartonien, et enfin MP17b au niveau de cet épisode inverse du C16, soit au début du Priabonien. Pour eux, MP19 et MP20 se succèdent à l'intérieur de la partie terminale du long épisode inverse du chron C13 (Priabonien terminal) et MP18 entre MP17b et MP19, pendant le chron C15. Cette corrélation est confirmée dans Barberá et al. (2001) pour le Priabonien terminal.

Les faunes-repères du dernier niveau du Priabonien (St-Capraise-d'Eymet) et du premier niveau du Rupélien (Soumailles) sont pauvres et ont été choisies uniquement parce qu'elles renfermaient à la fois des petits et des grands mammifères. Or, les stades évolutifs des quelques lignées représentées y sont peu discriminants, soit du MP précédent, soit du MP suivant (Aguilar et al., BiochroM'97, 1997 : 789-790). La discrimination du niveau MP20, qui repose essentiellement sur le stade terminal de Palaeotherium curtum (sous-espèce frohnstettense) n'est pas évidente (e.g., Hooker, 2010). Côté rongeurs, «Theridomys » bonduelli pourraît être un bon marqueur, mais sa position formelle au sein d'une lignée est encore mal établie ; l'espèce n'est connue que dans le Bassin-de-Paris et celui du Hampshire, ce qui limite sa valeur en terme de corrélations.

Cet état de fait a un impact certain sur la définition précise et les corrélations des faunes autour de la « Grande Coupure ». 
Plusieurs tentatives de corrélation ont été réalisées, à l'aide de la magnétostratigraphie et de la géochimie (Groupe de Solent, Whitecliff Bay, Ile de Wight, Angleterre ; Gale et al., 2006 ; Hooker et al., 2009) ou seulement la magnétostratigraphie (e.g., Barberá et al., 2001 ; Costa et al., 2011), en regard des lignées de mammifères. D'après ces derniers auteurs, le site de Santpedor est daté autour de 33,4 Ma. Cette localité renferme à la fois des rongeurs immigrants, comme les Cricetidae, ainsi que des autochtones, Gliridae et Theridomyidae. Ces derniers sont attribués à des espèces connues dans le niveau MP21 : T. aff. aquatilis et Pseudoltinomys gaillardi (Arbiol \& Saez, 1988). Les quelques dents conservées dans les collections de Sabadell (Catalogne, Espagne) ne portent ni numéro de catalogue, ni détermination (Minwer, pers. com.). Parmi elles, 15 dents sont des Theridomyidae (MV-L, observ. pers.). L'espèce la plus abondante et la plus grande (12 dents) est peut-être celle attribuée par Arbiol \& Saez, soit à $T$. aff. aquatilis, soit à $P$. gaillardi. Les quelques m1-2s et une m3 montrent un antésinuside (buccal donc), ce qui ne s'observe jamais chez «T. » aquatilis de Ronzon. Ces caractéristiques se retrouvent chez les Pseudoltinomys, mais entrent aussi dans la variation des molaires inférieures d'autres espèces de Theridomys comme « $T$. » golpei. Les dents supérieures auraient dû permettre de confirmer la présence de Pseudoltinomys mais, exceptée l'une des deux $\mathrm{P} 4 \mathrm{~s}$, les dents ont un patron plutôt de type Theridomys. Les deux autres dents (p4 et m1-2), plus petites, sont bien différentes et représentent une autre espèce pour l'instant indéfinie. Une révision de la faune de Santpedor s'impose donc, compte tenu de la place prise par ces rongeurs dans la discussion sur l'âge de cette localité et par suite, de l'âge de la « Grande Coupure » en Espagne.

La pauvreté des faunes de rongeurs de MP20 et MP21, alliée à la différenciation géographique constatée entre les théridomyoïdes du début de l'Oligocène ne facilite pas les corrélations fines. Il est aisé de repérer l'arrivée des immigrants (e.g., Atavocricetodon, Palaeosciurus, Lophallomys, Eomys et Steneofiber) et aussi de distinguer les stades évolutifs de certaines lignées lorsqu'elles sont représentées par suffisamment de dents, telles Pseudoltinomys cuvieri-gaillardi ou Blainvillimys langei-gregarius. Par contre, ces lignées ne sont pas présentes sur tout le territoire occupé par les Theridomyoidea en Europe Occidentale.

La " Grande Coupure » marquerait la base du Rupélien, actuellement datée de 33,9 Ma. Le repérage magnétostratigraphique du niveau MP21, supposé être représenté à Santpedor, au niveau de la partie supérieure du chron C13r, daterait ce NR aux environs de 33,4 Ma. Cela demande à être confirmé. Les NR mammaliens du Rupélien sont actuellement peu nombreux, au nombre de quatre (MP21 à MP24), sur une durée totale de l'étage de 6 Ma. Cependant, sur la base des degrés évolutifs de plusieurs lignées de Theridomyoidea, on peut intercaler au moins un niveau intermédiaire MP23/24 même s'il n'est pas encore formellement défini. Il correspondrait au niveau de la faune de St-Martin-de-Castillon (Vaucluse), avec notamment Protechimys truci (Hugueney, 1994 ; Vianey-Liaud, 1998). La localité-type de MP24 (Heimersheim) est située à la base des Cyrenen Mergel et est corrélée avec le Kasseler Meeressand, qui a fourni du nannoplancton de la zone NP24 (Bahlo, 1975). Ainsi, l'âge de MP24 se situe entre la limite Rupélien-Chattien et la base de la biozone NP24, soit entre 28,1 et 28,7 Ma.

Durant les cinq derniers millions d'années de l'Oligocène, entre 28,1 et 23,03 Ma, huit MP ont été définis (MP25 à MP30), du fait de l'évolution rapide de plusieurs lignées- guides, autorisant la discrimination de plusieurs stades évolutifs. Le concept de « lignée-guide 》 a été développé par Thaler (e.g., 1966). Il désigne ainsi les lignées d'espèces bien caractérisées, ayant valeur de marqueurs biochronologiques. Il s'agit essentiellement de lignées de Theridomyinae et d'Issiodoromyinae. Le dernier MP est corrélé avec des niveaux marins à Carry-le-Rouet (MP30/MN1) : il est antérieur au chron C6B et daté autour de $23 \mathrm{Ma}$ (e.g., Legendre \& Lévêque, 1997). MP28b est corrélé à la fois par la magnétostratigraphie autour du chron C7n (e.g., Schlunegger et al., 1996, Engesser \& Mödden, 1997) et radiochronologiquement (Mertz et al., 2007), autour de 24,5 Ma.

\section{Anciennes collections : validité des assemblages fossiles à la lumière de l'étude des Theridomyoidea et autres rongeurs}

$\mathrm{Au}$ cours du $19^{\text {ème }}$ siècle et jusqu'aux années 1940, peu de chercheurs paléontologues récoltaient eux-mêmes les fossiles qu'ils étudiaient en se préoccupant du contexte stratigraphique et géologique. La plupart du temps, les fossiles étaient collectés par des amateurs ou des carriers, au cours de travaux immobiliers, miniers ou de carrières. La dénomination du lieu de récolte était rarement précise et pouvait se perdre au fil du temps, au fur et à mesure des transferts, ventes ou échanges de collections. Quelques grandes zones ont produit de tels fossiles, qui sont parvenus pour étude auprès des scientifiques : en France, le Bassin-de-Paris, les Limagnes (Allier et HauteLoire), les Phosphatières du Quercy (Lot et Tarn-et-Garonne) ou les carrières près de Marseille ; en Suisse, des gisements dans la Molasse Suisse, ainsi que des remplissages karstiques (Le Mormont) ; en Allemagne, le Jura Souabe (e.g., remplissage karstique de Gaimersheim ; e.g., Freudenberg, 1941). Les rongeurs qui en sont issus sont à l'origine d'un nombre important de nouveaux taxons de Theridomyoidea.

La récolte sur le terrain par les chercheurs eux-mêmes s'est développée surtout à partir de la deuxième moitié du $20^{\text {ème }}$ siècle, alors qu'à la faveur des nombreux travaux portant sur les anciennes collections des interrogations sur la définition des taxons, leurs relations de parenté, leurs positions chronologiques relatives se multipliaient. Deux types de situations ont prévalu, jusqu'à la mise en route de fouilles scientifiques, qui sont illustrées ici par deux exemples.

-La qualité de préservation des fossiles issus des phosphatières du Quercy les rendaient incontournables tant pour appréhender la diversité des formes que pour les comparaisons nécessaires à l'établissement d'hypothèses phylogénétiques. La localité d'origine des fossiles quercinois était souvent le village ou le hameau le plus proche de la phosphatière exploitée (Bach, Escamps, Mouillac, etc.). De ce fait, un unique toponyme pouvait désigner des phosphatières différentes proches d'un même village ou hameau. Compte-tenu de la diversité des genres et espèces observés pour chacun de ces toponymes, les faunes quercinoises ont été considérées comme issues d'un long dépôt temporel. Il y aurait eu mélange d'espèces d'âges variés au cours du remplissage des phosphatières. L'impossibilité d'un contrôle chronologique des espèces affaiblissait la valeur des observations (e.g., de Bonis, 1974, à propos des travaux de Teilhard de Chardin sur les carnivores).

- Les sites de l'Oligocène des Limagnes illustraient une situation opposée : ils étaient considérés comme des sites bien localisés géologiquement, du fait qu'ils provenaient de localités incluses dans des séries sédimentaires réparties tout au long du fossé des Limagnes (Vallée de l'Allier et de la Loire, en Velay et Auvergne). 
Or, l'exploitation systématique conduite depuis les années 1960 dans les phosphatières du Quercy a permis d'obtenir des assemblages fauniques homogènes temporellement pour la majorité (de Bonis et al. 1973 ; Legendre et al., 1997), ou de repèrer clairement les réactivations éventuelles du remplissage après son dépôt initial (e.g., Vianey-Liaud, 2015). L'analyse de la variabilité des espèces et la reconnaissance de leur degré évolutif, notamment chez les Theridomyoidea, ont grandement contribué à établir ce fait (e.g., Aguilar et al., BiochroM'97, 1997). Pour les anciennes collections, les mélanges proviennent des collecteurs et ne reflètent pas des mélanges in situ consécutifs par exemple à une accumulation sur une longue durée du sédiment.

Les remarquables fossiles oligocènes des anciennes carrières de Limagne, répartis comme ceux du Quercy dans diverses institutions et collections particulières, ont fait l'objet de nombreuses études par les auteurs de la deuxième moitié du $19^{\text {ème }}$ siècle, et par la suite de révisions (e.g., Lavocat, 1951 ; Thaler, 1966). Il faut ajouter ici que la situation des faunes de Limagne ne diffère pas de celles du Quercy puisqu'il n'y a pas de contrôle géométrique possible de la position chronologique relative des gisements : le repérage chronologique des gisements repose alors le plus souvent sur les assemblages d'espèces et aussi sur le degré évolutif de certaines d'entre elles. Une mise au point des listes fauniques recense 34 localités et parmi elles 12 ont livré des Theridomyidae (Hugueney, 1997). Parmi elles, deux ont fait l'objet d'exploitations scientifiques « récentes »: St-Menoux (e.g., Viret \& Gauthier, 1950 ; Hugueney, 1980 ; MP26) et Coderet (e.g., Viret, 1926; Hugueney, 1969 ; MP30). Les faunes des sites de Ronzon, La-Sauvetat, Antoingtet Cournon ont été considérés comme des repères biochronologiques successifs, reposant en grande partie sur les lignées-guides de Theridomyidae (Thaler, 1964, 1965, 1966). La position relative de La-Sauvetat et d'Antoingt a été discutée à diverses reprises (Ginsburg, 1967 ; Rey, 1968 ; Thaler, 1972 ; Hugueney, 1986). Si l'on transpose au cadre actuel des MP l'échelle de zones de Thaler dans laquelle il place les localités oligocènes (e.g., Thaler, 1972 : tableau IV), La-Sauvetat se placerait au niveau MP22. En effet, Thaler contestait notamment la présence de Theridomys lembronicus parmi les rongeurs de ce site, espèce trouvée depuis dans d'autres sites et caractéristique du niveau MP25 (Thaler, 1972 : 422-423). Il souligne la nécessité " d'une interprétation critique » d'associations de rongeurs qui paraissent anachroniques, comme l'avait déjà fait Lavocat (1951), à propos d'Issiodoromys pseudanaema. Malgré cela, Hugueney (1986) s'est employée à démontrer la réalité d'un niveau évolutif unique des rongeurs de La-Sauvetat. Elle a révisé le matériel attribué à ce gisement dans les collections du Natural History Museum (NHMUK) à Londres et du Muséum national d'Histoire Naturelle (MNHN) à Paris. L'espèce Theridomys lembronicus y serait « abondante », mais serait de plus petite taille que celle d'Antoingt. Cette vingtaine de spécimens n'est pas figurée. Parmi eux, Thaler soulignait que le spécimen M25555 était sur un bloc portant la mention semieffacée « St-Yvoine » et qu'il fallait au minimum douter de la présence de l'espèce à La-Sauvetat.

Les spécimens appartenant à quatre autres espèces sont figurés (Hugueney, 1986 : figs. 1-10) :

1-Theridomys vassoni : le lectotype de cette espèce est une mandibule portant p4-m1, qui a seul servi à coder ce taxon dans notre analyse. Il se trouve rapproché de Paratheridomys, proche de $P$. margaritae et $P$. ludensis. Cette lignée est pour l'instant peu documentée et les implications biochronologiques encore limitées.
2-Blainvillimys blainvillei : LIM 250 (MNHN) et M25554 (NHMUK) : la comparaison de ces spécimens avec les $B$. blainvillei typiques, avec $B$. helmeri, $B$. gregarius et $B$. gemellus montre qu'ils sont plus proches de $B$. gemellus que de $B$. gregarius : le sIII n'est pas assez réduit et le sinuside pas assez long pour être un $B$. blainvillei ou même un $B$. helmeri. La dp4 de M25552 est d'un peu plus grande taille que celle de $B$. gregarius et montre une ouverture linguale du sI rappellant plus la dp4 de $B$. gemellus que celle de $B$. gregarius. En tout état de cause, il est clair qu'il ne s'agit pas de Blainvillimys blainvillei.

3-Issiodoromys minor : il s'agit bien plus vraisemblablement de Pseudoltinomys major (défini du niveau MP22 : Vianeyliaud 1976, 15-20, fig. 6 ; Vianey-Liaud \& Schmid, 2009). Comparé avec les spécimens type de La-Plante 2 (Vianeyliaud, 1976) et avec ceux de Cavalé (Vianey-liaud \& Schmid, 2009), le maxillaire M25549 (ancien M7811) s'en rapproche par la taille, la morphologie et le degré d'hypsodontie ; il en est de même pour la mandibule M25546b, sur laquelle on note aussi la forte réduction du lobe antérieur de la p4. Quant au spécimen M25531 de la collection du NHMUK, déterminé par Lavocat comme I. minor, la longueur de la dp4 relativement à $\mathrm{m} 1$, l'existence d'un antésinuside bien marqué, l'absence d'antéronide et d'antésinuside sur les molaires l'excluent définitivement d'Issiodoromys minor et le rapprochent plutôt de Blainvillimys.

4-Teniodus curvistriatus : l'espèce type est définie pour du matériel de La-Sauvetat. On la retrouve dans les localités de Balm et Coulou, considérées comme proches du niveau MP22, sur la base des stades évolutifs des autres Theridomyidae (Vianey-Liaud, 1998 : 273-276).

Ainsi, les stades évolutifs des spécimens provenant le plus sûrement de La-Sauvetat témoignent d'une nette différence entre la faune de ce site avec la faune de Theridomyidae d'Antoingt, d'où Toniodus est absent et où l'espèce référée à Blainvillimys est B. blainvillei, et celle à Theridomys est T. lembronicus. Une partie du matériel ancien attribué à La-Sauvetat provient vraisemblablement d'autres localités. Comme pour les anciennes collections du Quercy, des mélanges de collections en sont responsables. Par ailleurs, la connaissance améliorée des différentes lignées de Theridomyidae conduit à de nouvelles identifications et peut apporter les clés pour reconnaître les composantes d'un assemblage faunique réel.

\section{BILAN ET PERSPECTIVES}

Les rongeurs Theridomorpha ont vécu pendant une vingtaine de millions d'années. Apparus au tout début de l'Eocène moyen, ils se sont diversifiés à plusieurs reprises et ont subi un certain nombre d'extinctions au cours de leur histoire avant de disparaître à la fin de l'Oligocène. Bien que ces rongeurs se soient éteints, du fait de leur registre fossile relativement fourni et varié, montrant une diversité spécifique remarquable, ils offrent l'opportunité quasiment unique de suivre d'un bout à l'autre une radiation adaptative, sur un territoire restreint, à l'extrémité ouest de l'Europe Occidentale, au sud du 50 ème parallèle nord. Ainsi, ils représentent un cas exceptionnel permettant de mieux comprendre la dynamique évolutive d'un groupe de mammifères.

Les premières études sur ces rongeurs remontent à la première moitié du $19^{\text {ème }}$ siècle. Depuis, à plusieurs reprises, un état des lieux des connaissances a été dressé et des perspectives 
nouvelles se sont ouvertes, du fait de l'accroissement du nombre de fossiles et des progrès accomplis dans les méthodes d'étude et les concepts utilisés pour interpréter les fossiles. D'abord Schlosser (1884), puis Stehlin \& Schaub (1951), Lavocat (1951 et 1955) et Thaler (1962) en ont été les principaux acteurs. Notre démarche s'est inscrite dans cette continuité. Les objectifs annoncés en début de ce travail ont été en grande partie atteints, ouvrant de nouveaux horizons au développement de la connaissance de la radiation adaptative des Theridomorpha.

La morphologie dentaire a été décomposée en 315 caractères, assez détaillés pour pouvoir s'appliquer à la grande diversité des formes observées chez les Theridomopha tout au long du Paléogène. La terminologie utilisée est suffisamment générale pour pouvoir s'appliquer à l'ensemble des rongeurs, en permettant de décrire les homologies morphologiques.

L'analyse phylogénétique regroupant l'ensemble des espèces (AG) a permis de distinguer de façon formelle les Remyoidea des Theridomorpha, les deux taxons étant issus d'un ensemble Ischyromyiformes. Les Theridomorpha s'enracinent au sein des Ischyromyidae Reithroparamyinae Microparamyini. Au moins deux genres parmi eux, Hartenbergeromys et Pantrogna, peuvent être inclus à la base de l'infraordre des Theridomorpha, comme envisagé par Escarguel (1999), puis proposé par Marivaux et al. (2004). La phylogénie des Theridomyoidea obtenue est cohérente avec une grande partie des données antérieures et éclaire certaines zones d'ombre. Ainsi, les espèces de Protadelomys + Tardenomys ressortent comme les groupes frères successifs de l'ensemble Theridomyoidea. La dichotomie Pseudosciuridae - Theridomyidae est fortement soutenue. Ces deux ensembles divergent à la transition Lutétien/Bartonien et au moins deux genres de Pseudosciuridae montrent des patrons dentaires brachyodontes évoluant vers la sélénodontie (Tarnomys et Pseudosciurus). L'évolution de la morphologie dentaire des Theridomyidae a suivi plusieurs chemins, mais tous montrent, à des degrés divers, une augmentation dissymétrique de la hauteur des couronnes.

Parmi les premiers rameaux qui émergent, les genres regroupés dans la sous-famille des Issiodoromyinae montrent une évolution de l'émail dentaire de paucisérié à unisérié, comme c'est aussi le cas chez les Patriotheridomyinae (nouvelle sous-famille). Les Issiodoromys sont caractérisés par des prémolaires définitives qui demeurent plus courtes que les molaires, tandis que leurs prémolaires déciduales augmentent en longueur, une tendance qui va de pair avec l'accroissement de l'hypsodontie, de l'Eocène supérieur jusqu'à l'Oligocène supérieur. Chez les Patriotheridomyinae, les prémolaires définitives sont aussi allongées, voire plus que les molaires et les prémolaires déciduales nettement plus allongées que les molaires. Les Oltinomyinae priaboniens forment un clade bien défini, situé entre Issiodoromyinae et Patriotheridomyinae.

Les autres Theridomyidae ont un émail paucisérié, depuis le Priabonien au moins et jusqu'à la fin du Rupélien. Parmi eux, les Columbomyinae se singularisent par une faible surface des prémolaires déciduales et des prémolaires définitives et l'absence de mésolophide vrai aux dents inférieures. Les prémolaires définitives inférieures et supérieures des Theridomyinae sont aussi - voire plus - longues que les molaires, et les prémolaires déciduales nettement plus longues.

La systématique des Theridomyinae basaux demande à être révisée, les différents rameaux du premier clade des «Blainvillimys » comme ceux des «Theridomys " étant peu soutenus. La résolution de leur phylogénie impliquera à l'avenir une révision de ces taxons. La systématique des clades terminaux est mieux fixée, même si tout n'est pas résolu. Le clade Theridomys (major-octogesensis) montre une évolution parallèle de celle du clade des Blainvillimys oligocènes, avec accroissement de la semi-hypsodontie. Il s'en distingue par une taille plus grande, une moindre inclinaison des fûts des couronnes et par une généralisation et un approfondissement des synclinides supplémentaires (sI).

Dans les deux clades terminaux de Theridomyinae (Blainvillimys, ainsi que Protechimys et Archaeomys), l'émail des incisives passe parallèlement de paucisérié à pseudomultisérié. Dans ces clades, avec l'augmentation de la semihypsodontie, l'inclinaison du fût des couronnes dentaires est de plus en plus marquée, produisant des structures en lames parallèles obliques. Par ailleurs, les sinus et sinusides deviennent progressivement complètement traversants (sauf chez Toniodus), tandis que les petits synclinaux buccaux et synclinides linguaux réduisent fortement. Le cément apparaît aussi de façon convergente dans les flexus et flexides des taxons terminaux de Blainvillimys, Protechimys et Archaeomys. C'est parmi les formes oligocènes les plus hypsodontes, avec de grandes dents de lait, que le palais devient trapézoidal, étroit à l'avant et plus large à l'arrière : c'est le cas chez ces taxons terminaux mais aussi chez ceux d'Issiodoromys. Il y a vraisemblablement une contrainte développementale à cette évolution morphologique, en relation avec la forte augmentation de la taille des prémolaires déciduales et définitives relativement aux molaires.

Les chrono-espèces (sensu Simpson, 1951), traitées antérieurement comme des lignées évolutives (e.g., VianeyLiaud, 1976, 1979), apparaissent sous-forme de clades successifs (pour les mieux soutenues : e.g., Issiodoromys, Oltinomys-Ectropomys, Patriotheridomys, Theridomys, Blainvillimys et Protechimys). La plupart des espèces de ces genres émergent successivement (dichotomies successives) dans l'ordre stratigraphique, excepté pour Protechimys, où elles sont organisées en sens inverse. Cette dernière organisation est vraisemblablement le résultat de la forte convergence du dessin dentaire des dents inférieures de $P r$. major qui se range en rameau basal des Protechimys, avec celui des Archaeomys. L'espèce de Protechimys (Pr. truci) aux dents inférieures les moins transformées et les plus éloignées du schéma Archaeomys se place en branchement terminal. Par ailleurs, lorsque des genres sont représentés par plusieurs espèces contemporaines (e.g., clade des Blainvillimys oligocènes) mais relativement proches morphologiquement (e.g., B. gemellus et $B$. gregarius), la topologie ne permet pas de démêler les lignées envisagées.

L'histoire évolutive des Theridomorpha s'inscrit dans un contexte climatique, géographique et tectonique extrêmement changeant. Jusqu'à l'Eocène inférieur, des communications euraméricaines voire eurasiatiques ont pu avoir lieu par le nord, comme en témoignent la présence notamment des ischyromyoïdes. Puis l'Europe Occidentale est devenue insulaire à partir de l'Eocène moyen, creuset éminemment favorable à l'émergence de nouvelles espèces : les Theridomorpha apparaissent.

$\mathrm{Au}$ carrefour de mers plutôt froides (Mer du Nord et Atlantique) et d'une mer chaude (Téthys), en raison de mouvements tectoniques de grande ampleur [rotation de la plaque ibérique, remontée de l'Afrique, mouvements des diverses petites plaques (e.g., apulienne et adriatique) et formation des Pyrénées, des Alpes, des Dinarides et des Carpates], les contours paléogéographiques de l'Europe 
Occidentale ont varié considérablement entre l'Eocène moyen et la fin de l'Oligocène, qu'il s'agisse des contours des terres et des mers, ou bien de leurs altitude ou bathymétrie. En même temps, le climat global enregistrait des changements de forte amplitude (MECO il y a $41 \mathrm{Ma}$, Oi1, il y a 33,9 Ma, LOWE vers 24,5 Ma, sans compter plusieurs refroidissements accusés entre les deux, i.e., Oi1a à Oi2c). L'aridification et l'ouverture des milieux - même localement -, qui en sont un effet, ont très probablement joué un rôle dans le développement de l'hypsodontie chez de nombreuses lignées de Theridomyidae, tant au Priabonien (e.g., Patriotheridomys), qu'à partir du Rupélien (e.g., Issiodoromys et Protechimys). La répartition des genres de Theridomyoidea sur le territoire ouest-européen résulterait aussi de ces fluctuations de l'environnement dont la manifestation a dû également varier avec l'altitude et la latitude ; ainsi pourrait s'expliquer d'une part la dichotomie Pseudosciuridae-Theridomyidae, et d'autre part la répartition différenciée des Pseudosciurinae par rapport aux Columbomyinae ou Issiodoromyinae.

Malgré des différences de répartition paléogéographique des espèces de Theridomorpha suivant les époques, quelques genres plus ubiquistes ont grandement contribué à la biochronologie du Paléogène ouest-européen, du fait de leur évolution morphologique rapide. Ils ont été partie prenante de l'élaboration du système des niveaux-repères mammaliens du Paléogène européen (MP). Le bilan effectué ici est restreint à la période comprise entre l'Eocène moyen et l'Oligocène supérieur qui correspond à la durée d'existence des Theridomyoidea. Cette fenêtre temporelle montre des intervalles dans lesquels le nombre de MP est conséquent, avec des éléments d'étalonnage solides ; il s'agit d'abord du Chattien et, dans une moindre mesure, du Priabonien et du Bartonien. Plusieurs sites du Bassin de l'Ebre, comme Frontyana 1, sont susceptibles d'apporter des points d'étalonnage supplémentaires pour le Bartonien et le Priabonien. Les MP du Rupélien d'une part ne sont pas encore étalonnés et d'autre part demandent à être mieux argumentés tandis que des niveaux intermédiaires devraient être définis. Des progrès en la matière peuvent résulter de la comparaison des nombreuses faunes rapprochées des niveaux MP21, 22, $23,23 / 24$ et 24 . L'analyse morphologique, biométrique et morphométrique de plusieurs lignées de Theridomyidae, voire de Pseudosciuridae, y contribuerait efficacement. La situation du Lutétien est plus difficile car les Theridomyoidea y sont peu abondants. Il n'empêche qu'une révision des Protadelomys et des premiers Issiodoromyinae (Elfomys et Pseudoltinomys) devrait pouvoir améliorer la résolution.

A l'issue de ce bilan, il apparaît qu'un cadre phylogénétique relativement robuste est maintenant disponible pour les Theridomorpha. Des questions d'ordre systématique demeurent et devront être éclaircies à l'avenir. D'autres points demandent à être appuyés par des études approfondies de la variabilité morphologique des différents taxons concernés, qu'il s'agisse de Pseudosciuridae ou de Theridomyidae. Ces études seront également nécessaires pour préciser le contexte biochronologique (MP). Par ailleurs, l'étude de caractères morphologiques liés à d'autres traits d'histoire de vie (taille corporelle, régime alimentaire, comportement, activités, locomotion, écologie, etc.) de ces espèces éteintes n'en est qu'à ses débuts. Il s'agit notamment de l'examen de la morphologie des pétreux ou des éléments squelettiques postcrâniens, pour lesquels du matériel fossile est disponible, notamment au sein de l'abondant matériel provenant des fouilles effectuées depuis le milieu du $20^{\text {ème }}$ siècle dans les Phosphatières du Quercy.

\section{REMERCIEMENTS}

Nombreux sont celles et ceux qui ont contribué à des titres divers à la genèse de ce travail. Tenter de les nommer tous ferait courir le risque d'en oublier, mais nous tenons à mentionner $\dagger$ Bernard Comte, trop tôt disparu, avec qui les collaborations ont été précieuses et fructueuses.

Il nous est agréable aussi de remercier les collègues qui ont permis l'aboutissement de cette étude :

- Jerry Hooker pour son accueil au Natural History Museum, à Londres, où il a mis à notre disposition les pseudosciuridés qu'il avait étudiés ;

- à l'ISE-M, Bernard Marandat, qui nous a guidés dans le dédale des collections de rongeurs éocènes ; - Lionel Hautier, qui a fait partie des instigateurs de ce travail ; - Rodolphe Tabuce, qui a suggéré de réaliser une analyse cladistique, afin de voir comment se comportaient nos « lignées évolutives »; - Maéva Orliac et Fabrice Lihoreau, qui ont été de bon conseil, s'agissant de cette analyse ;

- Enfin, dans la phase finale, Rodolphe Tabuce et Anne-Lise Charruault ont réalisé les photographies des figures 1 et 16 .

Pour finir, il nous est agréable de remercier les deux rapporteurs anonymes qui ont eu le courage de relire ce long travail. Leurs nombreuses remarques, souvent pertinentes, auront contribué à son amélioration. Publication ISE-M 2016 - 273.

\section{BIBLIOGRAPHIE}

Adrover, R., Feist, M., Ginsburg, L., Guérin, C., Hugueney, M., Moissenet, E., 1983. Les formations continentales paléogènes de la Sierra Palomera (Province de Teruel, Espagne) et leur place dans la biostratigraphie tertiaire des chaînes ibériques orientales. Bulletin de la Société Géologique de France 7, XXV, 421-431. doi:10.2113/gssgfbull.S7-XXV.3.421

Aguilar, J.-P., Legendre, S., Michaux, J., 1997. Biochronologie mammalienne du cénozoïque en Europe et domaines reliés. In: Aguilar, J.-P., Legendre, S., Michaux, J. (Eds.), Actes du Congrès BiochroM'97. Mémoires et Travaux de l'Institut de Montpellier de l'Ecole Pratique des Hautes Etudes, Montpellier 21, pp. 1-818.

Alston, E. R., 1876. On the classification of the order Glires. Proceedings of the zoological Society of London 44, 61-98.

Anadon, P., Vianey-Liaud, M., Cabrera, L., Hartenberger, J.-L., 1987. Gisements à vertébrés du Paléogène de la zone orientale du bassin de l'Ebre et leur apport à la stratigraphie. Paleontologia i Evolucio 21, 117-131.

Andó, M., Shiraishi, S., Uchida, T. A., 1985. Food habits of the Japanese giant flying squirrel, Petaurista leucogenys. Journal of Faculty of Agriculture, Kyushu University 29, 189-202.

Antunes, M. T., Casanovas, M. L., Cuesta, M. A., Checa, L., Santafé, J. V., Agustí, J., 1997. Eocene mammals from Iberian Peninsula. In: Aguilar, J.-P., Legendre, S., Michaux, J. (Eds.), Actes du Congrès BiochroM'97. Mémoires et Travaux de l'Institut de Montpellier de l'Ecole Pratique des Hautes Etudes, Montpellier 21, 337-352.

Arbiol, S., 1993. Revision de la fauna de micromamiferos del yacimiento oligocénico de Can Quaranta (Campins, Vallès Oriental). Paleontologia i Evolucio 26-27, 107-120.

Arbiol, S., Agusti, J., Hugueney, M., 1997. A new species of Theridomys (Rodentia, Mammalia) from the upper Oligocene from the Ebro basin (NE Spain). Geobios 30, 447-451.

Arbiol, S., Saez, A., 1988. Sobre la edad oligocenica inferior del yacimiento de Santpedor (Cuenca del Ebro, provincia de Barcelona). Acta Geologica Hispanica 23, 47-50.

Astibia, H., Aranburu, A., Pereda Suberbiola, X., Murelaga, X., Sesé, 
C., Cuesta, M. A., Moyà-Solà, S., Baceta, J. I., Badiola, A., Köhler, M., 2000. Un nouveau site à vertébrés continentaux de l'Eocène supérieur de Zambrana (Bassin de Miranda-Treviño, Alava, Pays Basque). Geobios 33, 233-248.

Aymard, A., 1850. Mammifères fossiles des calcaires du Puy. Annales de la Société d'Agriculture, Sciences, Arts et Commerce du Puy 14, 80-86.

Bahlo, E., 1975. Die Nagetierfauna von Heimersheim bei Alzey (Rheinhessen, Westdeutschland) aus dem Grenzbereich Mittel / Oberoligozän und ihre stratigrafische Stellung. Abhandlungen des Hessichen Landesamtes für Bodenforschung 71, 1-182.

Barberà, X., Cabrera, L. Y., Marzo, M., Parès, J. M., Agusti, J., 2001. A complete terrestrial Oligocene magnetobiostratigraphy from the Ebro Basin, Spain. Earth and Planetary Science Letters 187, 1-16.

Becker, D., 2009. Earliest record of rhinocerotoids (Mammalia : Perissodactyla) from Switzerland: systematics and biostratigraphy. Swiss Journal of Geosciences 102, 489-504. doi:10.1007/s00015-009-1330-4

Black, C. C., Dawson, M., 1989. Papers on Fossil Rodents. In Honor of Albert Elmer Wood. Sciences Series Natural History Museum of Los Angeles County 33, 1-193.

Blondel, C., 2001. The Eocene - Oligocene ungulates from Western Europe and their environment. Paleogeography, Paleoclimatology, Paleoecology 168, 125-139. doi:10.1016/ S0031-0182(00)00252-2

Blondel, C., 2005. New data on the Cainotheriidae (Mammalia, Artiodactyla) from the early Oligocene of south-western France. Zoological journal of the Linnean society 144, 145166. doi:10.1111/j.1096-3642.2005.00166.x

Bonilla-Salomón, I., Minwer-Barakat, R., Vianey-Liaud, M., 2016. Middle Eocene rodents from Sant Jaume de Frontanyà-1 (Eastern Pyrenees, Northern Spain) and biochronological implications. Journal of Vertebrate Paleontology 36, in press. doi:10.1080/02724634.2016.1121149

Bonis, L. de, 1964. Étude de quelques mammifères du Ludien de La Débruge (Vaucluse). Annales de Paléontologie 50, 119-154.

Bonis, L., de, Crochet, J. Y., Rage, J. C., Sigé, B., Sudre, J., VianeyLiaud, M. 1973. Nouvelles faunes de vertébrés oligocènes des phosphorites du Quercy. Bulletin du Muséum National d'Histoire Naturelle 3, 174, 105-113.

Bonis, L. de, 1974. Premières données sur les carnivores fissipèdes provenant des fouilles récentes dans le Quercy. Palaeovertebrata 6, 27-32.

Bosma, A. A., 1974. Rodent Biostratigraphy of the Eocene Oligocene transitional strata of the Isle of Wight. Utrecht Micropaleontological Bulletins, Special Publication, pp. $1-128$

Bosma, A. A., Insole, A. N., 1976. Pseudosciuridae (Rodentia, Mammalia) from Osborne beds (Late Eocene), Isle of Wight, England. Proceedings of the Koninklijke Nederlandse Akademie van Wetenschappen B 79, 1-8.

Bosma, A. A., Schmidt-Kittler, N., 1972. Ectropomys exiguus n. gen. n. sp., member of the Oltinomyinae n. subfamily (Theridomyidae, Rodentia), from Paleogene deposits of the Isle of Wight (England) and Southern Germany. Proceedings of the Koninklijke Nederlandse Akademie van Wetenschappen B 75, 181-192.

Bravard, A., 1844. Considérations sur la distribution des mammifères terrestres fossiles dans le département du Puy-de-Dôme, Thibaud-Landriot Frères.

Bruijn, H. de, Sondaar, P. Y., Sanders, E. A. C., 1979. On a new species of Pseudoltinomys (Theridomyidae, Rodentia) from the Paleogene of Mallorca. Proceedings of the Koninklijke Nederlandse Akademie van Wetenschappen 82, 1-10.

Brunet, M., Jéhenne, Y., Ringeade, M., 1977. Note préliminaire concernant la découverte d'une faune et d'une flore du niveau de Ronzon dans l'Oligocène inférieur du Bassin d'Aquitaine. Geobios 10, 109-112.

Butler, P. M., 1985. Homologies of molar cusps and crests, and their bearing on assessments of rodent phylogeny. In: Luckett,
W. P., Hartenberger, J.-L. (Eds), Evolutionary Relationships Among Rodents, A Multidisciplinary Analysis. Plenum Press, New York, pp. 381-401. doi:10.1007/978-1-4899-0539-0

Cavelier, C., 1965. Le Sannoisien de Sannois (Seine-et-Oise) dans le cadre du Bassin de Paris et sa signification stratigraphique. Bulletin de la Société Géologique de France 7, 228-238.

Collinson, M. E., Hooker, J. J., 2003. Paleogene vegetation of Eurasia: framework for mammalian faunas. DEINSEA 10, 41-83.

Comte, B., Sabatier, M., Marandat, B., Vianey-Liaud, M., 2012. Les rongeurs de Chéry-Chartreuve et Rocourt-Saint-Martin, Aisne, France). Leur place parmi les faunes de l'Eocène moyen d'Europe. Palaeovertebrata 37, 167-271. doi:10.18563/ pv.37.4-5.167-271

Comte, B., Vianey-Liaud, M., 1989. Eomyidae (Rodentia) de l'Oligocène d'Europe occidentale. Palaeontographica A, 3391.

Costa, E., Garcés, M., López-Blanco, M., Serra-Kiel, J., Bernaola, G., Cabrera, L., Beamud, E., 2013. The Bartonian-Priabonian marine record of the eastern south Pyrenean foreland basin (NE Spain): a new calibration of the larger foraminifers and calcareous nannofossil biozonation. Geologica Acta 11, 177193. doi:10.1344/105.000001779

Cox, P. G., Hautier, L., 2015. Evolution of the Rodents: Advances in Phylogeny, Functional Morphology and Development. Cambridge University Press. Cambridge.

Cramer, B. S., Toggweiler, J. R., Wright, J. D., Katz, M. E., Miller, K. G., 2009. Ocean overturning since the Late Cretaceous: inferences from a new benthic foraminiferal isotope compilation. Paleoceanography 24, 1-14. doi:10.1029/2000PA000528

Cuenca, G., Canudo, J.-J., Laplana, C., Andres, J. A., 1992. Bio y cronoestratigrafia con mamiferos en la Cuenca Terciaria del Ebro: ensayo de sintesis. Acta Geologica Hispanica 27, 127 143.

Cuenca-Bescós, G., 1998. Los pseudoesciúridos (Rodentia) del Eoceno Superior de la cuenca lacustre de Sossís (Pirineos). In: López-Martínez, N., Casanovas-Cladellas, M. L., Daams, R. (Eds), Geología Y Paleontología Del Eoceno de La Pobla de Segur. Universitat de Lleida, pp. 181-233.

De Laizer, M., De Parieu, J.-B. E., 1839. Paleomys arvernensis. L'Echo du Monde Savant 408, 67-68.

Dechaseaux, C., 1965. Artiodactyles des phosphorites du Quercy : 1- Etude sur le genre Dichodon. Annales de Paléontologie (Vertébrés) 51, 91-108.

Dechaseaux, C., 1967. Artiodactyles des phosphorites du Quercy : 2- Etude sur le genre Xiphodon. Annales de Paléontologie (Vertébrés) 55, 198-248.

Dehm, R., 1937. Neue tertiäre Spaltenfüllungen im südlichen Fränkischen Jura. Zentralblatt für Mineralogie, Geologie und Paläontologie. Abteilung B 9, 349-369.

Depéret, C., 1906. Los vertebrados deI Oligocenico inferior de Tarregá (prov. de Lerida). Real Academia de Ciencias y Artes de Barcelona 3, 401-425.

Depéret, C., 1917. Monographie de la faune de mammifères fossiles du Ludien inférieur d'Euzet-les-Bains (Gard). Annales de l'Université de Lyon 1, 1-228.

Dercourt, J., Ricou, L. E., Vrielynck, B., 1993. Atlas Tethys Palaeoenvironmental Maps: Explanatory Notes. Atlas. Gauthier-Villars, Paris.

Engesser, B., Mödden, C., 1997. A new version of the biozonation of the Lower Freshwater Molasse (Oligocene and Agenian) of Switzerland and Savoy on the basis of fossil Mammals. In: Aguilar, J.-P., Legendre, S., Michaux, J. (Eds.), Actes du Congrès BiochroM'97. Mémoires et Travaux de l'Institut de Montpellier de l'Ecole Pratique des Hautes études, Montpellier 21, 475-499.

Escarguel, G., 1997. Une nouvelle méthode morphométrique pour l'étude des dents jugales inférieures de mammifères : application au genre Protadelomys (Theridomyidae, Rodentia, Mammalia). Münchner Geowissenschaftliche Abhandlungen A, 65-72.

Escarguel, G., 1998. Protadelomys maximini nov. sp. (Rodentia, 
Mammalia) : apport à la connaissance du genre Protadelomys et implications biochronologiques. Geobios 31, 371-383. doi:10.1016/S0016-6995(98)80020-X

Escarguel, G., 1999. Les rongeurs de l'Eocène inférieur et moyen d'Europe Occidentale. Systématique, phylogénie, biochronologie et paléobiogéographie des niveaux-repères MP7 à MP14. Palaeovertebrata 28, 89-351.

Fabre, P.-H., Hautier, L., Douzery, E., 2015. A synopsis of rodent molecular phylogenetics, systematics and biogeography. In: Cox, P. G., Hautier, L. (Eds.), Evolution of the Rodents. Advances in Phylogeny, Functional Morphology and Development. Cambridge University Press, Cambridge, 5, pp. 19-69. doi:10.1017/CBO97811073601500

Fejfar, O., 1987. A Lower Oligocene mammalian fauna from Dětan and Dvérce (NW Bohemia, Czechoslovakia). In: SchmidtKittler., N. (Ed.), International Symposium on Mammalian Biostratigraphy and Paleoecology of the European Paleogene, Mainz. Münchner Geowissenschaftliche Abhandlungen A 10, 253-264.

Filhol, H., 1872a. Sur les carnassiers et les chéiroptères dont on trouve les débris fossiles dans les gisements de phosphorite de Caylus, Crégols, Concots. Comptes Rendus de l'Académie des Sciences, Paris 75, 92-94.

Filhol, H., 1872b. Note relative à la découverte dans les gisements de phosphate de chaux du Lot d'un mammifère fossile nouveau. Bulletin de la Société des Sciences physique et naturelle, Toulouse 1, 92-94.

Filhol, H., 1877. Recherches sur les phosphorites du Quercy : étude des fossiles qu'on y rencontre et spécialement des mammifères. Annales de la société géologique de France 8, 1-340.

Filhol, H., 1879. Etude des mammifères fossiles de Saint-Gérandle-Puy (Allier). Annales des Sciences Géologiques, Paris 8, $1-340,28 \mathrm{pl}$.

Filhol, H., 1880. Découverte de mammifères nouveaux dans les dépôts de phosphate de chaux du Quercy. Comptes Rendus de l'Académie des Sciences, Paris 90, 1579-1580.

Filhol, H., 1882. Découverte de quelques nouveaux genres de mammifères fossiles dans les dépôts de phosphate de chaux de Quercy. Comptes Rendus de l'Académie des Sciences, Paris 94, 138-139.

Franzen, J. L., 1968. Revision der Gattung Palaeotherium Cuvier 1804 (Palaeotheriidae, Perissodactyla, Mammalia). PhD, Albert-Ludwigs-Universität zu Freiburg.

Franzen, J. L., 2005. The implications of the numerical dating of the Messel fossil deposit (Eocene, Germany) for mammalian biochronology. Annales de Paléontologie 91, 329-335.

Freudenberg, H., 1941. Die oberoligocänen Nager von Gaimersheim bei Ingolstadt und ihre Verwandten. Palaeontographica. Abteilung, A092, 99-164.

Freudenthal, M., 1997. Paleogene rodent faunas from the province of Teruel (Spain). In: Aguilar, J.-P., Legendre, S., Michaux, J. (Eds.), Actes Du Congrès BiochroM'97. Mémoires et Travaux de 1'Institut de Montpellier de l'Ecole Pratique des Hautes Etudes, Montpellier 21, 397-415.

Freudenthal, M., Lacomba, J. I., Martinez-Salanova, J., Sacristàn, A., 1990. Nueva sucesion de niveles con micromamiferos en el oligoceno de Montalbàn (Prov. Teruel, España). Acta Salmaticensia 68, 133-140.

Friant, M., 1934. L'évolution de la dentition des Mammifères. Revue de Stomatologie 36, 607-617.

Gad, J., 1987. Theridomys brachydens $\mathrm{n}$. sp., a new Theridomys species from Möhren (South Germany). In: Schmidt-Kittler., N. (Ed.), International Symposium on Mammalian Biostratigraphy and Paleoecology of the European Paleogene, Mainz. Münchner Geowissenschaftliche A, 269-272.

Gale, A. S., Huggett, J. M., Pälike, H., Laurie, E., Hailwood, E. A., Hardenbol, J., 2006. Correlation of Eocene-Oligocene marine and continental records: orbital cyclicity, magnetostratigraphy and sequence stratigraphy of the Solent Group, Isle of Wight, UK. Journal of the Geological Society, London 163, 401-415. doi:10.1144/0016-764903-175
Garzon Heydt, G., Lopez Martinez, N., 1978. Los roedores fosiles de los Barros (Avila). Datacion del Paleogeno continental en el Sisterna Central. Estudios geologicos 34, 571-575.

Gervais, P., 1848. Zoologie et paléontologie françaises (animaux vertébrés) ou nouvelles recherches sur les animaux vivants et fossiles de France. Arthus Bertrand, Paris

Ginot, S., Hautier, L., Marivaux, L., Vianey-Liaud, M., 2016. Ecomorphological analysis of the astragalo-calcaneal complex in rodents and inferences of locomotor behaviours in extinct rodent species. PeerJ 4, e2393. doi:10.7717/peerj.2393

Ginsburg, L., 1967. L'âge relatif des gisements de mammifères de la Limagne d'Auvergne. Comptes Rendus Sommaires de la Société Géologique de France 7, 325.

Gradstein, F. M., Ogg, G., Schmitz, M., 2012. The Geologic Time Scale 2012. Elsevier B.V.

Hartenberger, J.-L., 1968. Les Pseudosciuridae (Rodentia) de l'Eocène moyen et le genre Masillamys Tobien. Comptes Rendus de l'Académie des Sciences, Paris 267, 1817-1820.

Hartenberger, J.-L., 1969. Les Pseudosciuridae (Mammalia, Rodentia) de l'Eocène moyen de Bouxwiller, Egerkingen et Lissieu. Palaeovertebrata 3, 27-61.

Hartenberger, J.-L., 1971a. La systématique des Theridomyoidea (Rodentia). Comptes Rendus de l'Académie des Sciences, Paris 273, 1917-1920.

Hartenberger, J.-L., 1971b. Contribution à l'étude des genres Gliravus et Microparamys (Rodentia) de l'Éocène d'Europe. Palaeovertebrata 4, 97-135.

Hartenberger, J.-L., 1973a. Les rongeurs de l'Éocène d'Europe. Leur évolution dans leur cadre biogéographique. Bulletin du Muséum National d'Histoire Naturelle, Paris 132, 49-70.

Hartenberger, J.-L., 1973b. Etude systématique des Theridomyoidea (Rodentia) de l'Éocène supérieur. Mémoires de la Société Géologique de France NSLII, 1-5, 1-76.

Hartenberger, J.-L., 1990. L'origine des Theridomyoidea (Mammalia, Rodentia) : données nouvelles et hypothèses. Comptes Rendus de l'Académie des Sciences 311, 1017-1023.

Hartenberger, J.-L., Louis, P., 1976. Nouveaux Rongeurs dans l'Éocène supérieur de Grisolles (Aisne). Geobios 9, 81-95.

Hartenberger, J.-L., Schmidt-Kittler, N., 1976. Un nouveau genre de Theridomyoidea (Mammalia, Rodentia) dans les Phosphorites du Quercy. Mitteilungen der Bayerischen Staatssammlung für Paläontologie und Historische Geologie, Munchen 16, 65-69.

Hautier, L., Clavel, J., Lazzari, V., Gomes Rodrigues, H., VianeyLiaud, M., 2010. Biomechanical changes and remodeling of the masticatory apparatus during mammalian evolution: the case of the Issiodoromyinae (Rodentia). Palaios 25, 6-13. doi:10.2110/palo.2009.p09-047r

Hautier, L., Cox, P. G., Lebrun, R., 2015. Grades and clades among rodents: the promise of geometric morphometrics. In: Cox, P. G., Hautier, L. (Eds.), Evolution of the Rodents. Advances in Phylogeny, Functional Morphology and Development. Cambridge University Press, Cambridge 5, 277-299. doi:10.1017/CBO97811073601500

Heissig, K., 1987. Changes in the rodent and ungulate fauna in the Oligocene fissure fillings of Germany. In: SchmidtKittler., N. (Ed.), International Symposium on Mammalian Biostratigraphy and Paleoecology of the European Paleogene, Mainz. Münchner Geowissenschaftliche A 10, 101-108.

Heissig, K., Schmidt-Kittler, N., 1972. Neue Lagomorphen-Funde aus dem Mitteloligozän. Mitteilungen der Bayerischen Staatssammlung für Paläontologie und Historische Geologie $16,83-93$.

Hensel, R., 1856. Beitträge zur Kenntnis fossiler Säugetiere. Zeitschrift für Deutsche Geologie Gesellschaft 8, 660-704.

Hoffstetter, R., Lavocat, R., 1970. Découverte dans le Déseadien de Bolivie des genres pentalophodontes appuyant les affinités africaines des rongeurs caviomorphes. Comptes Rendus de l'Académie des Sciences, Paris 271, 172-175.

Hooker, J. J., 1986. Mammals from the Bartonian (middle/late Eocene) of the Hampshire Basin, southern England. Bulletin of the British Museum of Natural History, Geology series 39, 
191-478.

Hooker, J. J., 1991. Two new pseudosciurids (Rodentia, Mammalia) from the English Late Eocene, and their implications for phylogeny and speciation. Bulletin of the British Museum of Natural History, Geology series 47, 35-50.

Hooker, J. J., 2010. The "Grande Coupure" in the Hampshire Basin, UK: taxonomy and stratigraphy of the mammals on either side of this major Paleogene faunal turnover. In: Whittaker, J. E., Hart, M. B. (Eds), Micropaleontology, Sedimentary Environments and Stratigraphy: A Tribute to Dennis Curry (1912-2001). The Micropalaeontological Society, Special Publications, pp. 147-215. doi:10.1144/TMS004.8

Hooker, J. J., Grimes, S. T., Mattey, D. P., Collinson, M. E., Sheldon, N. D., 2009. Refined correlation of the UK Late EoceneEarly Oligocene Solent Group and timing of its climate history. Geological Society of America 452, 179-195. doi:10.1130/2009.2452(12)

Hooker, J. J., Weidmann, M., 2000. The Eocene mammal faunas of Mormont, Switzerland: systematic revision and resolutions of dating problems. Schweizerischen Paläontologische Abhandlungen 120, 1-143

Hooker, J. J., Weidmann, M., 2007. A diverse rodent fauna from the middle Bartonian (Eocene) of Les Alleveys, Switzerland: snapshot of the early theridomyid radiation. Swiss Journal of Geosciences 100, 469-493. doi:10.1007/s00015-007-1241-1

Hubbard, R. N. L. B., Boulter, N. C., 1983. Reconstruction of Paleogene climate from palynological evidence. Nature 301, 147-150. doi:10.1038/301147a0

Huchon, D., Catzeflis, F. M., Douzery, E. J., 2000. Variance of molecular datings, evolution of rodents and the phylogenetic affinities between Ctenodactylidae and Hystricognathi. Proceedings of the Royal Society of London B 267, 393-402. doi:10.1098/rspb.2000.1014

Huchon, D., Chevret, P., Jordan, U., Kilpatrick, C. W., Ranwez, V., Jenkins, P. D., Schmitz, J., 2007. Multiple molecular evidences for a living mammalian fossil. Proceedings of the National Academy of Sciences 104, 7495-7499. doi:10.1073/ pnas.0701289104

Huchon, D., Douzery, E. J., 2001. From the Old World to the New World: a molecular chronicle of the phylogeny and biogeography of hystricognath rodents. Molecular Phylogenetics and Evolution 20, 238-251. doi:10.1006/mpev.2001.0961

Hugueney, M., 1969. Les rongeurs (Mammalia) de l'Oligocène supérieur de Coderet-Bransat (Allier). PhD, Faculté des Sciences de Lyon.

Hugueney, M., 1980. La faune de mammifères de l'Oligocène moyen de Saint-Menoux (Allier). 1- Rongeurs (Mammalia Rodentia). Revue scientifique du Bourbonnais 57-71.

Hugueney, M., 1986. Les mammifères de La Sauvetat (Puy-de-Dome). Leur place dans l'Oligocène moyen. Revue scientifique du Bourbonnais 68-82.

Hugueney, M., 1994. Theridomys truci de l'Oligocène de SaintMartin-de-Castillon (Vaucluse, France), nouvelle espèce du genre Theridomys (Rodentia, Mammalia) et sa relation avec la lignée de Theridomys lembronicus. Scripta Geologica 104, 115-127.

Hugueney, M., 1997. Biochronologie mammalienne dans le Paléogène et le Miocène inférieur du Centre de la France : synthèse réactualisée. In: Aguilar, J.-P., Legendre, S., Michaux, J. (Eds.), Actes du Congrès BiochroM'97. Mémoires et Travaux de l'Institut de Montpellier de l'Ecole Pratique des Hautes Etudes, Montpellier 21, 417-430.

Hugueney, M., Moissenet, E., Adrover, R., 1992. Une nouvelle lignée du genre Columbomys (Mammalia, Rodentia, Theridomyidae) dans l'Oligocène supérieur d'Espagne. Comptes Rendus de l'Académie des Sciences, Paris 315, 379-385.

Jaeger, J.-J., 1989. L'évolution de la pentalophodontie chez les rongeurs caviomophes (Mammalia, Rodentia). Geobios Mémoire Spécial 12, 235-244.

Jaeger, J.-J., Hartenberger, J.-L., 1975. Pour l'utilisation systématique de niveaux-repères en biochronologie mammalienne. 3ème
Réunion Annuelle Des Sciences de La Terre, Montpellier, pp. 201.

Jourdan, C., 1837. Mémoire sur quelques mammifères nouveaux. Comptes Rendus des Séances de l'Académie des Sciences 15, 521-524.

Kócsis, L., Ozsvárt, P., Becker, D., Ziegler, R., Scherler, L., Codrea, V., 2014. Orogeny forced terrestrial climate variation during the late Eocene-early Oligocene in Europe. Geology 35673, 1-4. doi:10.1130/G35673.1

Kotler, B. P., 1985. Owl predator on desert rodents which differ in morphology and behavior. Journal of Mammalogy 66, 824828. doi: $10.2307 / 1380824$

Lacomba, J. I., Morales, J., 1987. Los mamiferos del Oligoceno superior de Carrascosa del Campo (Prov. Cuenca, Espana). In: Schmidt-Kittler, N. (Ed.), International Symposium on Mammalian Biostratigraphy and Paleoecology of the European Paleogene, Mainz. Münchner Geowissenschaftliche Abhandlungen A10, 289-299.

Lartet, E., 1869. Sur le Trechomys bonduelli et deux autres rongeurs fossiles de 1'Eocène parisien. Annales des Sciences Naturelles, Paris 12, 151-166.

Lavocat, R., 1951. Révision de la faune des mammifères oligocènes d'Auvergne et du Velay. Sciences et Avenir, Paris.

Lavocat, R., 1955. Quelques progrès récents dans la connaissance des rongeurs fossiles et leurs conséquences sur divers problèmes de systématique, de peuplement et d'évolution. Colloque International CNRS 60, 77-85.

Legendre, S., 1987. Les immigrations de la "Grande Coupure" sontelles contemporaines en Europe Occidentale ? In: SchmidtKittler, N. (Ed.), International Symposium on Mammalian Biostratigraphy and Paleoecology of the European Paleogene, Mainz. Münchner Geowissenschaftliche Abhandlungen A 10, 141-147.

Legendre, S., Lévêque, F., 1997. Etalonnage de l'échelle biochronologique mammalienne du Paléogène d'Europe occidentale: vers une intégration à l'échelle globale. In: Aguilar, J.-P., Legendre, S., Michaux, J. (Eds.), Actes du Congrès BiochroM'97. Mémoires et Travaux de l'Institut de l'Ecole Pratique des Hautes Etudes, Montpellier 21, 461-473.

Legendre, S., Sigé, B., Astruc, J. G., Bonis, L. De, Crochet, J., Denys, C., Godinot, M., Hartenberger, J.-L., Lévêque, F., Marandat, B., Mourer-Chauviré, C., Rage, J., Remy, J. A., Sudre, J., Vianey-Liaud, M., 1997. Les phosphorites du Quercy : 30 ans de Recherche. Bilan et perspectives. Geobios Mémoire Spécial 20, 331-345.

Lenz, O. K., Wilde, V., Mertz, D. F., Riegel, W., 2015. New palynologybased astronomical and revised ${ }^{40} \mathrm{Ar} /{ }^{39} \mathrm{AR}$ ages for the Eocene maar lake of Messel (Germany). International Journal of Earth Sciences 104: 873-889. doi:10.1007/s00531-014-1126-2

Lopez, N., Thaler, L., 1975. Sur le plus ancien lagomorphe européen et la "Grande Coupure" Oligocène de Stehlin. Palaeovertebrata 6, 243-251.

Luckett, P. W., Hartenberger, J.-L., 1985. Evolutionary Relationships Among Rodents. A Multidisciplinary Analysis. Plenum Press, New York.

Major, F., 1873. Nagerüberreste aus Bohnerzen Süddeutschlands und der Schweiz. Palaeontographica 75-130.

Man, E. De, Simaeys, S. Van, 2004. Late Oligocene warming event in the southern North Sea Basin: benthic foraminifera as paleotemperature proxies. Netherlands Journal of Geosciences $83,227-239$.

Marivaux, L., Adaci, M., Bensalah, M., Gomes Rodrigues, H., Hautier, L., Mahboubi, M., Mebrouk, F., Tabuce, R., VianeyLiaud, M., 2011. Zegdoumyidae (Rodentia, Mammalia), stem anomaluroid rodents from the early to middle Eocene of Algeria (Gour Lazib, Western Sahara): new dental evidence. Journal of Systematic Palaeontology 9, 563-588. doi:10.1080 /14772019.2011.562555

Marivaux, L., Essid, E. M., Marzougui, W., Khayati Ammar, H., Adnet, S., Marandat, B., Merzeraud, G., Tabuce, R., VianeyLiaud, M., 2014. A new and primitive species of Protophiomys 
(Rodentia, Hystricognathi) from the late middle Eocene of Djebel El Kébar, Central Tunisia. Palaeovertebrata 38, 1-e2. doi:10.18563/pv.38.1.e2

Marivaux, L., Essid, E. M., Marzougui, W., Khayati Ammar, H., Merzeraud, G., Tabuce, R., Vianey-Liaud, M., 2015. The early evolutionary history of anomaluroid rodents in Africa: new dental remains of a zegdoumyid (Zegdoumyidae, Anomaluroidea) from the Eocene of Tunisia. Zoologica Scripta 44, 117-134. doi:10.1111/zsc.12095

Marivaux, L., Vianey-Liaud, M., Jaeger, J.-J., 2004. High-level phylogeny of early Tertiary rodents: dental evidence. Zoological Journal of the Linnean Society 142, 105-134. doi:10.1111/j.1096-3642.2004.00131.x

Marivaux, L., Vianey-Liaud, M., Welcomme, J.-L., Jaeger, J.-J., 2002. The role of Asia in the origin and diversification of hystricognathous rodents. Zoologica Scripta 31, 225-239. doi:10.1046/j.1463-6409.2002.00074.x

Martin, T., 1999. Evolution of incisor enamel microstructure in Theridomyidae (Rodentia). Journal of Vertebrate Paleontology 19, 550-565. doi:10.1080/02724634.1999.10011164

Mayo, N. A., 1987. New Theridomyidae (Rodentia, Mammalia) in the Oligocene of the Molasse of Switzerland and Savoy. Eclogae Geologicae Helvetiae 80, 995-1085.

Meng, J., Li, C., Ni, X., Wang, Y., Beard, K. C., 2007. A new Eocene rodent from the lower Arshanto Formation in the Nuhetingboerhe (Camp Margetts) Area, Inner Mongolia. American Museum Novitates 3569, 1-18. doi:10.1206/00030082(2007)536[1:ANERFT]2.0.CO;2

Mertz, D. F., Renne, P. R., 2005. A numerical age for the Messel fossil deposit (UNESCO World Heritage Site) derived from ${ }^{40} \mathrm{Ar} /{ }^{39} \mathrm{Ar}$ dating on a basaltic rock fragment. In: Harms, F. J., Schaal, S. (Eds), Current Geological and Paleontological Research in the Messel Formation. Courier Forschungsinstitut Senckenberg 255, 67-75.

Mertz, D. F., Renne, P. R., Wuttke, M., Mödden, C., 2007. A numerically calibrated reference level (MP28) for the terrestrial mammal-based biozonation of the European Upper Oligocene. International Journal of Earth Sciences 96, 353361. doi:10.1007/s00531-006-0094-6

Meulenkamp, J. E., Sissingh, W., 2003. Tertiary palaeogeography and tectonostratigraphic evolution of the Northern and Southern Peri-Tethys platforms and the intermediate domains of the African-Eurasian convergent plate boundary zone. Palaeogeography, Palaeoclimatology, Palaeoecology 196, 209-228. doi:10.1016/S0031-0182(03)00319-5

Michaux, J., 1964. Diagnoses de quelques Paramyidés de l'Éocène inférieur de France. Comptes Rendus Sommaires des Séances de la Société Géologique de France 4, 153.

Michaux, J., 1968. Les Paramyidae (Rodentia) de l'Oligocène inférieur du Bassin de Paris. Palaeovertebrata 1, 135-193.

Misonne, X., 1957. Mammifères oligocènes de Hoogbutsel et de Hoeleden. Bulletin de l'Institut Royal des Sciences Naturelles de Belgique 33, 1-15.

Mödden, C., 1993. Revision der Archaeomyini Schlosser (Rodentia, Mammalia) des Europäischen Oberoligozän. Schweizerische Palaeontologische Abhandlungen 115, 1-83.

Mödden, C., Vianey-Liaud, M., 1997. The Upper Oligocene tribe Archaeomyini (Theridomyidae, Rodentia, Mammalia): systematics and biostratigraphy. In: Aguilar, J.-P., Legendre, S., Michaux, J. (Eds.), Actes du Congrès BiochroM'97. Mémoires et Travaux de l'Institut de l'Ecole Pratique des Hautes Etudes, Montpellier 21, 361-374.

Mörs, T., Kalthoff, D., 2010. Anew species of Amphilagus (Mammalia: Lagomorpha) from the Late Oligocene lake deposits of Enspel (Westerwald, Germany). Palaeobiodiversity and Palaeoenvironments 90, 83-98. doi:10.1007/s12549-0090020-6

Nury, D., 1988. L’Oligocène de Provence méridionale, stratigraphie, dynamique sédimentaire, reconstitutions paléogéographiques. Documents du BRGM 180, 1-395.

Ogg, J. G., Ogg, G., Gradstein, F. M., 2008. The Concise Geologic
Time Scale (Vol. 1). Cambridge University Press, Cambridge. Patterson, B., Wood, A. E., 1982. Rodents from the Deseadan Oligocene of Bolivia and the relationships of the Caviomorpha. Museum of Comparative Zoology, Harvard University 149, 7, 372-543.

Peláez-Campomanes, P., 1996. Upper Eocene rodents from the Almazàn basin (Soria, Spain). Eclogae Geologicae Helvetiae 89, 1363-1386.

Peláez-Campomanes, P., 2000. Mammalian faunas from the Paleogene of the Sierra Palomera (Teruel, Spain). Journal of Paleontology 74, 336-348. doi:10.1666/0022-3360(2000)074<0336:MFFT $\mathrm{PO}>2.0 . \mathrm{CO} ; 2$

Peláez-Campomanes, P., Lopez-Martinez, N., 1996. Strange Eocene rodents from Spain. Palaeovertebrata 25, 323-338.

Peláez-Campomanes, P., Pena, A. de la, Lopez-Martinez, N., 1989. Primeras faunas de micromamiferos del Paleogeno de la Cuenca del Duero. Studia Geologica Salmanticensia, Salamanca, Volumen especial, 135-150.

Picot, L., Becker, D., Cavin, L., Pirkenser, C., Lapaire, F., Ruber, G., Hochuli, P. A., Spezzaferi, S., Berger, J. P., 2008. Sédimentologie et paléontologie des paléoenvironnements côtiers rupéliens de la molasse marine rhénane dans le Jura suisse. Swiss Journal of Geosciences 101, 483-513. doi:10.1007/s00015-008-1275-z

Pictet, F.-J., Humbert, A., 1869. Mémoire sur les animaux vertébrés trouvés dans le terrain sidérolithique du canton de Vaud et appartenant à la faune éocène. Matériaux pour la Paléontologie Suisse 5, 121-197.

Pomel, A., 1853. Catalogue méthodique et descriptif des vertébrés fossiles découverts dans le bassin hydrographique supérieur de la Loire et surtout dans la vallée de son affluent principal de l'Allier. Baillière, J. J., Paris.

Poschmann, M., Schindler, T., Uhl, D., 2010. Fossil-Lagerstätte Enspel - a short review of current knowledge, the fossil association, and a bibliography. Palaeobiodiversity and Palaeoenvironments 90, 3-20.

Quer, N., Agusti, J., 2010. A new genus of rodents (Remyidae, Mammalia) from the Iberian Eocene. Palaeontology 53, 5358. doi:10.1111/j.1475-4983.2009.00917.x

Rey, R., 1968. L'Oligocène à partir des mollusques continentaux. Annales Guébhard 44, 162-196.

Russell, D. E., Hartenberger, J.-L., Pomerol, C., Sen, S., SchmidtKittler, N., Vianey-Liaud, M., 1982. Mammals and stratigraphy: the Paleogene of Europe. Palaeovertebrata Mémoire Extraordinaire, 1-77.

Scherzinger, A., Franz, M., Heizmann, E. P. J., Hofmann, F., 2004. New fissure fillings from northern Hegau and the area around Sigmaringen (western Swabian Alb, SW Germany). Stuttgarter Beiträge zur Naturkunde 354, 1-45.

Schettino, A., Turco, E., 2011. Tectonic history of the western Tethys since the Late Triassic. Geological Society of America Bulletin 123, 89-105. doi:10.1130/B30064.1

Schlosser, M., 1884. Die Nager des Europäischen Tertiärs: nebst Betrachtungen über die Organisation und die geschichtliche. Palaeontographica 21, 1-143.

Schlunegger, F., Burbank, D. W., Matter, A., Engesser, B., Modden, C., 1996. Magnetostratigraphic calibration of the Oligocene to Middle Miocene (30-15 Ma) mammal biozones and depositional sequences of the Swiss Molasse Basin. Eclogae Geologicae Helvetiae 89, 753-788.

Schmidt-Kittler, N., 1971. Odontologische Untersuchungen an Pseudosciuriden (Rodentia, Mammalia) des Alttertiärs. Bayerische Akademie der Wissenschaften, MathematischNaturwissenschaftliche Klasse Abhandlungen NF 150, 1-135.

Schmidt-Kittler, N., 1984. Pattern analysis of occlusal surfaces in hypsodont herbivores and its bearing on morpho-functional studies. Proceedings of the Koninklijke Nederlandse Akademie van Wetenschappen. Series B 87, 453-480.

Schmidt-Kittler, N., 1987. International Symposium on Mammalian Biostratigraphy and Paleoecology of the European Paleogene - Mainz. Münchner Geowissenschaftliche Abhandlungen A $10,17-20$. 
Schmidt-Kittler, N., Vianey-Liaud, M., 1975. Les relations entre les faunes de rongeurs d'Allemagne du Sud et de France pendant l'Oligocène. Comptes Rendus de l'Académie des Sciences, Paris 281, 511-514.

Schmidt-Kittler, N., Vianey-Liaud, M., 1987. Morphometric analysis of the genus Issiodoromys (Theridomyidae, Rodentia) of the European Oligocene. Proceedings of the Koninklijke Nederlandse Akademie van Wetenschappen, Series B. 90, 281-306.

Schmidt-Kittler, N., Vianey-Liaud, M., Mödden, C., Comte, B., 1997. New data for the correlation of mammal localities in the European Oligocene: biochronological relevance of the Theridomyidae. In: Aguilar, J.-P., Legendre, S., Michaux, J. (Eds.), Actes du Congrès BiochroM'97. Mémoires et Travaux de l'Institut de l'Ecole Pratique des Hautes Etudes, Montpellier 21, 375-396.

Sigé, B., Hugueney, M., Crochet, J.-Y., Legendre, S., Mourer-Chauviré, C., Rage, J.-C., Simon-Coinçon, R., 1998. Baraval, nouvelle faune de l'Oligocène inférieur (MP22) des phosphorites du Quercy : apport à la signification chronologique des remplissages karstiques. Bulletin de la Société d'Histoire Naturelle de Toulouse 134, 85-90.

Sigé, B., Vianey-Liaud, M., 1979. Impropriété de la Grande Coupure de Stehlin comme support d'une limite Eocène-Oligocène. Newsletters on Stratigraphy 8, 79-82. doi:10.1127/ nos $/ 8 / 1979 / 79$

Simpson, G. G., 1951. The species concept. Evolution 5, 285-298.

Stehlin, H. G., 1909. Remarques sur les faunules de mammifères des couches éocènes et oligocènes du Bassin de Paris. Bulletin de la Société Géologique de France 4, 488-520.

Stehlin, H. G., Schaub, S., 1951. Die trigonodontie der simplicidentaten Nager. Schweizerische Palaeontologische Abhandlungen 67, 1-385.

Sudre, J., 1978. Les artiodactyles de l'Eocène moyen et supérieur d'Europe occidentale : systématique et évolution. Mémoires et Travaux de l'Institut de l'Ecole Pratique des Hautes Etudes, Montpellier 7, 1-229.

Sudre, J., 1988. Apports à la connaissance de D. robertiana GERVAIS 1848-1852 (Mammalia, Artiodactyla) du Lutétien; considérations sur l'évolution des Dichobunidés. Courier Forschungsinstitut Senckenberg 107, 409-418.

Swofford, D. L., 2002. PAUP* Phylogeny Analysis Using Parsimony (* and other methods). Sunderland, Massachusetts.

Thaler, L., 1962. Premiers résultats d'une recherche systématique des dents de rongeurs, par lavage de marnes de l'Oligocène, en Bas-Languedoc. Comptes Rendus Sommaires de la Société Géologique de France 10, 315.

Thaler, L., 1964. Sur l'utilisation des mammifères dans la zonation du Paléogène de France. Mémoires du BRGM 28, 985-989.

Thaler, L., 1965. Une échelle de zones biochronologiques pour les Mammifères du Tertiaire d'Europe. Compte Rendu Sommaire des Séances de la Société Géologique de France 4, 118-119.

Thaler, L., 1966. Les rongeurs fossiles du Bas-Languedoc dans leurs rapports avec l'histoire des faunes et la stratigraphie du Tertiaire d'Europe. Mémoires du Muséum National d'Histoire Naturelle, Paris N.S. C, XV, 1-295.

Thaler, L., 1969. Rongeurs nouveaux de l'Oligocène moyen d'Espagne. Palaeovertebrata 2, 191-207.

Thaler, L., 1972. Datation, zonation et mammifères. Mémoires du BRGM 77, 411-424

Thaler, L., 1983. Image paléontologique et contenu biologique des lignées évolutives. Colloques Internationaux CNRS 330, $327-$ 335 .

Tobien, H., 1954. Nagerreste aus dem Mitteleoziin von Messel bei Darmstadt. Notizbl. Hessichen Landesamtes für Bodenforschung 82, 13-29.

Tobien, H., 1955. Eine stampische Kleinssäuger fauna aus der Grenzregion Schleichsand/Cyrenenmergel von Heimersheim bei Alzey (Rheinhessen). Zeitschrift für deutsche geologie Gesellschaft 106, 565.

Tobien, H., 1963. Zur gebiß-entwicklung tertiärer Lagomorphen
(Mamm.) Europas Notizblatt des hessischen Landesamtes für Bodenforschung zu Wiesbaden 91, 15-35.

Tobien, H., 1972. Mikromammalier aus dem altertiären menalienton von Nordhessen. Notizblatt des hessischen Landesamtes für Bodenforschung zu Wiesbaden 100, 7-40.

Vandenberghe, N., Hilgen, F. J., Speijer, R. P., 2012. The Paleogene period. In: Gradstein, F. M., Ogg, J. G., Schmitz, M., Ogg, G. (Eds.), The Geologic Time Scale, Chap. 28. Elsevier B.V., pp. 855-921. doi:10.1016/B978-0-444-59425-9.00028-7

Vianey-Liaud, M., 1969. Rongeurs de l'Oligocène moyen provenant de nouvelles fouilles dans les phosphorites du Quercy. Palaeovertebrata 2, 209-239.

Vianey-Liaud, M., 1972a. L'évolution du genre Theridomys à l'Oligocène moyen. Intérêt biostratigraphique. Bulletin du Muséum National d'Histoire Naturelle, Paris 98, 295-372.

Vianey-Liaud, M., 1972b. Un cas de parallélisme intragénérique: l'évolution du genre Theridomys (Rodentia, Theridomyidae) à l'Oligocène moyen. Comptes Rendus de l'Académie des Sciences, Paris 274, 1007-1010.

Vianey-Liaud, M., 1974. Les Rongeurs de l'Oligocène inférieur d'Escamps. Palaeovertebrata. 6, 197-241.

Vianey-Liaud, M., 1975. Caractéristiques évolutives des rongeurs de l'Oligocène d'Europe Occidentale. Colloque International CNRS 218, 765-776.

Vianey-Liaud, M., 1976. Les Issiodoromyinae (Rodentia, Theridomyidae) de l'Eocène supérieur à l'Oligocène supérieur en Europe Occidentale. Palaeovertebrata 7, 1-115.

Vianey-Liaud, M., 1977. Nouveaux Theridomyidae du Paléogène d'Europe Occidentale. Comptes Rendus de l'Académie des Sciences, Paris 284, 1277-1280.

Vianey-Liaud, M., 1979. Evolution des rongeurs à l'Oligocène en Europe Occidentale. Palaeontographica A 166, 136-236.

Vianey-Liaud, M., 1985. Nouvelles quantification de l'hypsodontie chez les Theridomyidae : l'exemple de Theridomys ludensis nov. sp. Palaeovertebrata $15,159-172$.

Vianey-Liaud, M., 1989. Parallélisme chez les Theridomyinae (Rodentia) de l'Oligocène : étude de deux nouvelles espèces des genres Theridomys et Blainvillimys. Neue Jahrbuch für Geologie und Paläontologie, Abhandlungen 178, 203-241.

Vianey-Liaud, M., 1991. Un Theridomyidae (Mammalia, Rodentia) très hypsodonte dans l'Eocène supérieur (MP16) du Quercy. Geobios Mémoire Spécial, 13, 79-88.

Vianey-Liaud, M., 1998. La radiation des Theridomyidae (Rodentia) à l'Oligocène inférieur : modalités et implications biochronologiques. Geologica et Palaeontologica 32, 253285.

Vianey-Liaud, M., 2015. Parallelism in the evolution of dental pattern and systematic implications: the case of Protechimys major Schlosser, 1884 (Theridomyinae, Rodentia, Mammalia) and its associated rodents. Comptes Rendus Palevol 14, 451-470. doi:10.1016/j.crpv.2014.12.008

Vianey-Liaud, M., Comte, B., Lévêque, F., 1995. Le Garouillas et les sites contemporains (Oligocène MP25) des phosphorites du Quercy (Lot, Tarn et Garonne, France) et leurs faunes de vertébrés : rongeurs. Palaeontographica A 236, 257-326.

Vianey-Liaud, M., Hautier, L., Marivaux, L., 2015. Morphological disparity of the postcranial skeleton in rodents and its implications for palaeobiological inferences: the case of the extinct Theridomyidae (Rodentia, Mammalia). In: Cox, P. G., Hautier, L. (Eds.), Evolution of the Rodents. Cambridge University Press, Cambridge, pp. 539-588. doi:10.1017/ CBO97811073601500

Vianey-Liaud, M., Lebrun, R., 2013. New data about the oldest European Lagomorpha: description of the new genus Ephemerolagus nievae gen. nov. and sp. nov. Spanish Journal of Palaeontology 28, 3-16.

Vianey-Liaud, M., Michaux, J., 2003. Evolution graduelle à l'échelle géologique chez les rongeurs fossiles du Cénozoïque européen. Comptes Rendus Palevol 2, 455-472. doi:10.1016/j. crpv.2003.09.026

Vianey-Liaud, M., Ringeade, M., 1993. La radiation des 
Theridomyidae (Rodentia) hypsodontes à l'Eocène supérieur. Geobios 26, 455-495.

Vianey-Liaud, M., Schmid, B., 2009. Diversité, datation et paléoenvironnement de la faune de mammifères oligocènes de Cavalé (Quercy, SO France) : contribution de l'analyse morphométrique des Theridomyinae (Mammalia, Rodentia). Geodiversitas 31, 909-941. doi:/10.5252/g2009n4a909

Vianey-Liaud, M., Schmidt-Kittler, N., 1987. Biostratigraphie de l'Oligocène d'Europe : importance des lignées guides de rongeurs Theridomyidae et particulièrement des Issiodoromyinae. In: Schmidt-Kittler, N. (Ed.), International Symposium on Mammalian Biostratigraphy and Paleoecology of the European Paleogene, Mainz. Münchner Geowissenschaftliche A 10, 211-216.

Vianey-Liaud, M., Schmidt-Kittler, N., Peláez-Campomanes, P., 1994. Pairomys et Ectropomys : la fin d'une ambiguïté ; mise au point sur les Oltinomyinae et les Remyinae (Rodentia, Theridomyinae). Palaeovertebrata 23, 119-152.

Viret, J., 1926. Les faunes de mammifères de l'Oligocène supérieur de la Limagne bourbonnaise. Annales de l'Université de Lyon N.S. $1,1-328$

Viret, J., Gauthier, H., 1950. Sur l'âge des calcaires de Saint-Menoux (Allier). Comptes Rendus de l'Académie des Sciences, Paris 230, 221-223.

Vollmayr, T., 1966. Oberoligozäne Gliridae (Rodentia, Mammalia) aus der süddeutschen Faltenmolasse. Mitteilungen der Bayerischen Staatssammlung für Paläontologie und Historische Geologie 6, 65-107.

Webster, D. B., Webster, M., 1980. Morphological adaptation of the ear in the rodent family Heteromyidae. American Zoologist $20,247-254$.
Wilson, D. E., Reeder, D. M., 2005. Mammal Species of the World: A Taxonomic and Geographic Reference. Johns Hopkins University Press, Baltimore.

Wolfe, J. A., 1978. A paleobotanical interpretation of Tertiary climates in the Northern Hemisphere: data from fossil plants make it possible to reconstruct Tertiary climatic changes, which may be correlated with changes in the inclination of the Earth's rotational axis. American Scientist 66, 694-703.

Wood, A. E., 1937. Rodentia. Part II in the mammalian fauna of the White River Oligocene. In: Scott, W. B., Jepsen, G. L. (Eds.), Transactions of the American Philosophical Society. Philadelphia, pp. 155-269.

Wood, A. E., 1962. The early Tertiary rodents of the family Paramyidae. Transactions of the American Philosophical Society, Philadelphia N.S. 52, 1-261.

Wood, A. E., Wilson, R. W., 1936. A suggested nomenclature for the cusps of the cheek teeth of rodents. Journal of Paleontology $10,388-391$.

Zachos, J. C., Pagani, M., Sloan, L., Thomas, E., Billups, K., 2001. Trends, rhythms, and aberrations in global climate $65 \mathrm{Ma}$ to Present. Science 292, 686-693. doi:10.1126/science.1059412

Zachos, J. C., Dickens, G. R., Zeebe, R. E., 2008. An early Cenozoic perspective on greenhouse warming and carbon-cycle dynamics. Nature 451, 279-283. doi:10.1038/nature06588

Zittel, K. A., 1893. Handbuch der paleontologie. I Abteilung, Paleozoologie, IV Band, Vertebrata (Mammalia). R. Oldenbourg, Munchen und Berlin. 